\title{
Investigation of Emissions and Fuel Economy for the Integrated Bus Information System
}

Jun Tu

West Virginia University

Follow this and additional works at: https://researchrepository.wvu.edu/etd

\section{Recommended Citation}

Tu, Jun, "Investigation of Emissions and Fuel Economy for the Integrated Bus Information System" (2013). Graduate Theses, Dissertations, and Problem Reports. 302.

https://researchrepository.wvu.edu/etd/302

This Dissertation is protected by copyright and/or related rights. It has been brought to you by the The Research Repository @ WVU with permission from the rights-holder(s). You are free to use this Dissertation in any way that is permitted by the copyright and related rights legislation that applies to your use. For other uses you must obtain permission from the rights-holder(s) directly, unless additional rights are indicated by a Creative Commons license in the record and/ or on the work itself. This Dissertation has been accepted for inclusion in WVU Graduate Theses, Dissertations, and Problem Reports collection by an authorized administrator of The Research Repository @ WVU.

For more information, please contact researchrepository@mail.wvu.edu. 
Investigation of Emissions and Fuel Economy for the Integrated Bus Information System

Jun Tu

Dissertation submitted to the College of Engineering and Mineral Resources at West Virginia University in partial fulfillment of the requirements

for the degree of

\author{
Doctor of Philosophy \\ in \\ Mechanical Engineering
}

W. Scott Wayne, Ph.D., Chair

Mario G. Perhinschi, Ph.D.

Nigel N. Clark, Ph.D.

Hailin Li, Ph.D.

John W. Zondlo, Ph.D.

Department of Mechanical and Aerospace Engineering

Morgantown, West Virginia

2013

Keywords: Emissions, Transit Buses, Fuel Economy, Emission Models, Duty Cycles, Cycle Generation 


\begin{abstract}
Investigation of Emissions and Fuel Economy for the Integrated Bus Information System
\end{abstract}

Jun Tu

The primary objective of this study was to investigate emissions and fuel economy, and develop an Integrated Bus Information System (IBIS) for the Federal Transit Administration (FTA). IBIS included the development of transit fleet emissions models to assist transit agencies in evaluating the emissions implications of new transit vehicle procurements. Compared with existing models, the IBIS prediction model was intended to be less complicated but have sufficient accuracy to achieve its task as a vehicle procurement analysis tool.

Fuel economy (FE) and distance specific emissions (g/mile) were evaluated and predicted by the IBIS model, including carbon monoxide (CO), carbon dioxide $\left(\mathrm{CO}_{2}\right)$, oxides of nitrogen (NOx), hydrocarbons (HC), and particulate matter (PM). Most data used in this study were based on chassis dynamometer testing conducted by West Virginia University (WVU), considering that chassis dynamometer test cycles could reflect the actual vehicle operations.

Many factors affect emissions and fuel economy, including vehicle parameters, fuel type, engine parameters, road conditions, ambient conditions and driving characteristics. Since driving characteristics significantly affected emissions and fuel economy, to determine the model inputs, correlation and regression studies between distance specific emissions, fuel economy and driving characteristics were performed. Results showed that average speed with idle (or average speed), percentage idle, stops per mile, standard deviation of vehicle speed, and kinetic intensity were the most influential parameters in driving characteristics and should be considered as the main driving parameters for the development of the predictive fleet emissions model.

A micro-trip based method was used throughout this research. A genetic algorithm (GA) was implemented to generate numerous new virtual cycles, to expand the cycle and emission database and to investigate transit operation characteristics encountered in the real-world. Then, the cycle generation method was applied to multiple representative buses tested with different types of fuel and powertrain technologies, to acquire the emissions and fuel economy data on over 350 newly generated virtual cycles. In addition, emissions testing was conducted over 
selected virtual cycles and validated the cycle generation method. It suggested that fuel consumption, $\mathrm{CO}_{2}$ and $\mathrm{NOx}$ emissions were not sensitive to microtrip history (sequence).

Based on this expanded dataset, multiple predictive backbone models were developed in certain model year (MY) groups for different fuel or propulsion system types (conventional and hybrid). The backbone models were validated with an additional dataset. For example, in terms of average percent errors, if using three cycle parameters as IBIS model inputs, emissions and FE of a MY 2008 60-foot CNG bus were predicted within 6\% for FE, 6-8\% for $\mathrm{CO}_{2}, 16-18 \%$ for CO, and 22-29\% for HC. Emissions and FE of a MY 2008 40-foot hybrid bus were predicted within $7 \%$ for $\mathrm{FE}, 8-10 \%$ for $\mathrm{CO}_{2}$, and 7-17\% for NOx. Multiple correction factors were developed to improve the models by introducing additional non-cycle parameters including vehicle weight, MY groups, and after-treatment technologies.

A case study compared the IBIS model with the Emission FACtors (EMFAC) model developed by California Air Resources Board (CARB). Comparison results agreed well for CO, NOx and PM for MY 2000 diesel buses and agreed well for CO for MY 2006 diesel buses. On average the IBIS model agreed well with the EMFAC model in terms of $\mathrm{CO}_{2}$ and fuel economy. In addition, both models showed that emissions and fuel economy did not change as the vehicle aged. 


\section{ACKNOWLEDGEMENTS}

Upon concluding my study and research at West Virginia University (WVU), I would like to take this opportunity to appreciate those who helped and supported me.

I must express my most sincere appreciation to my advisor Dr. Scott Wayne for his great guidance throughout my Ph.D. study and research at WVU. When I was stressed out and frustrated, Dr. Wayne always showed tremendous support, understanding, encouragement and patience. Dr. Wayne's strict scientific research attitude, professionalism and kindness have deeply impressed and inspired me and will positively affect my future career as well as the rest of my life. In addition, it is an honor to have Dr. Nigel Clark, Dr. Mario Perhinschi, Dr. Hailin Li, and Dr. John Zondlo on my committee. I am very grateful for their constructive suggestions and comments on my research work.

I would like to acknowledge Dr. Mario Perhinschi and his graduate students Chris Marlowe and Sergio Tamayo for developing computation tools for the IBIS project which were very helpful for this research. I still remember those weekly meetings we spent together with Dr. Scott Wayne and Dr. Nigel Clark to discuss the project and keep it moving forward. In addition, I appreciate Zac Luzader for uploading the IBIS searchable database and emissions model on the website.

I appreciate CAFEE's emissions testing lab crew for collecting data for this research. I especially thank David McKain for his support and patience to provide me with emissions testing data.

I want to thank graduate program assistant Debbie Willis, graduate program directors Dr. Jacky Prucz, Dr. Nithi Sivaneri, and Dr. Victor Mucino for their great academic guidance as well as thanks for assistance with student related things which made my student life easier at WVU.

I thank CAFEE’s graduate students: Yuebing Wu, Feng Zhen, Jairo Sandoval, Francisco Posada, Idowu Olatunji, Clay Bell, ABM S Khan, Lijuan Wang, Wei Qi, Manoharan Thiagarajan, Marc Besch, and many others for spending time with me to discuss interesting topics as well as for the friendship we built. 
I am grateful to my family for their support. I specially thank my wife Hua for her love, understanding and inspiration, and my beloved son Ocean for bringing more joy into my life.

Finally, I would like to acknowledge the US Department of Transportation Federal Transit Administration for sponsoring this project. 


\section{TABLE OF CONTENTS}

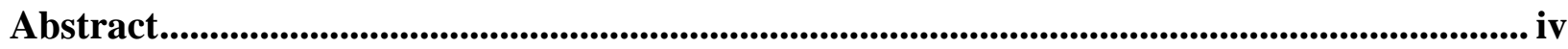

Acknowledgements ......................................................................................................... iv

Table of Contents ............................................................................................................................... vi

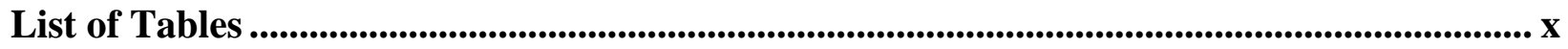

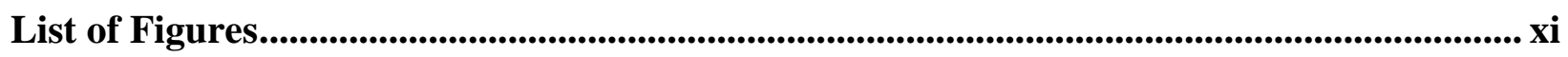

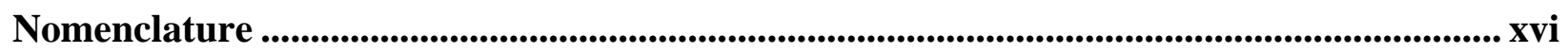

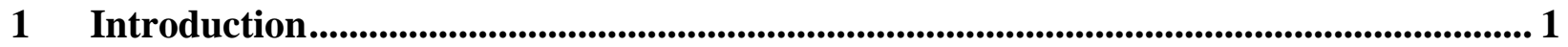

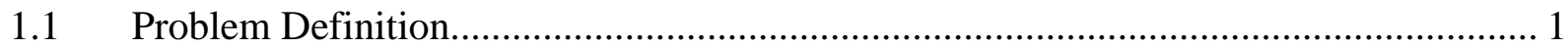

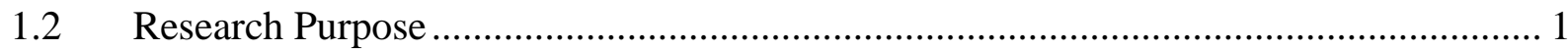

1.3 Research Objectives and Approaches ........................................................................ 2

1.4 Anticipated Contributions ........................................................................................... 4

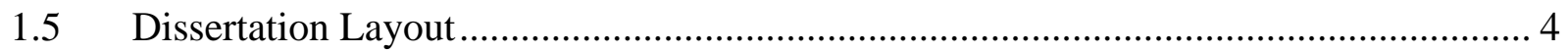

2 Literature Review .................................................................................................................. 6

2.1 Characterization of Emissions and Fuel Economy …………....................................... 6

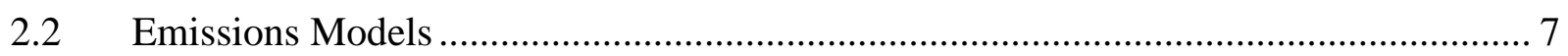

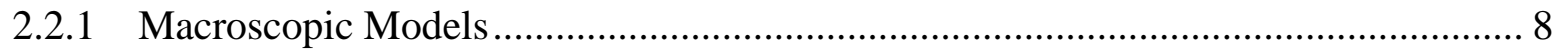

2.2.1.1 MOBILE Model and EMFAC Model.............................................................. 8

2.2.1.2 Other Macroscopic Models........................................................................... 9

2.2.2 Microscopic Models................................................................................... 9

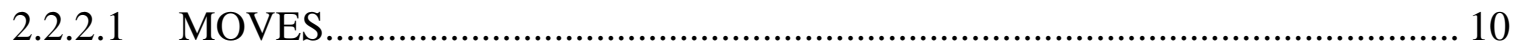

2.2.2.2 Modal Binning Method............................................................................... 13

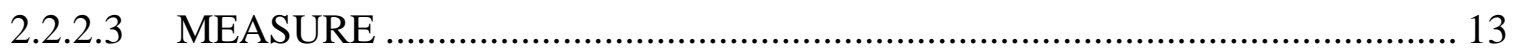

2.2.2.4 Speed-Acceleration Method............................................................................ 14

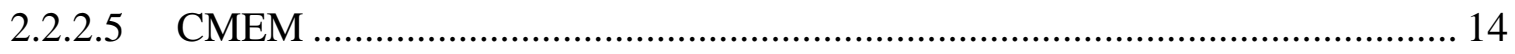

2.2.2.6 Neural Network Based Models ........................................................... 15

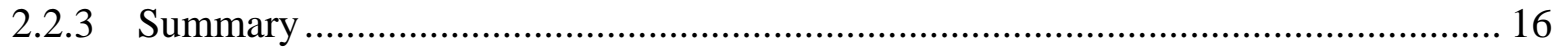

2.3 Chassis Dynamometer Cycles for Emissions Testing ……………………….............. 16

2.3.1 Central Business District Cycle .................................................................................. 17 
2.3.2 Braunschweig City Cycle …………………........................................................... 18

2.3.3 The European Transient Cycle................................................................................ 18

2.3.4 The New York Bus Cycle ................................................................................... 20

2.3.5 The New York Composite Cycle ........................................................................ 20

2.3.6 The Manhattan Cycle ............................................................................................... 21

2.3.7 The Heavy-Duty Urban Dynamometer Driving Schedule......................................... 22

2.3.8 The Washington Metro Area Transit Authority Cycle …………………………….... 23

$2.4 \quad$ Cycle Metrics ............................................................................................................. 24

$2.5 \quad$ Cycle Development.............................................................................................. 27

3 IBIS Development Strategies …........................................................................................... 29

3.1 IBIS Model Inputs............................................................................................... 30

3.2 IBIS Model Outputs .............................................................................................. 32

3.3 General Architecture of IBIS Model Development ....................................................... 32

3.4 Testing Facilities and Data Collection ......................................................................... 35

3.4.1 Description of WVU Testing Facilities ..................................................................... 35

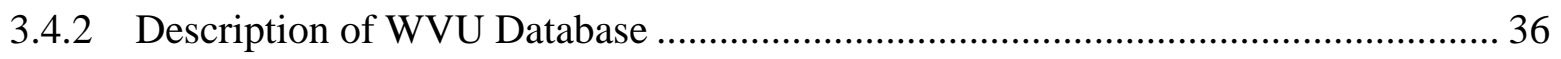

4 Correlation Analysis of Driving Characteristics Effects...................................................... 38

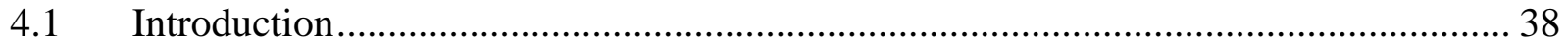

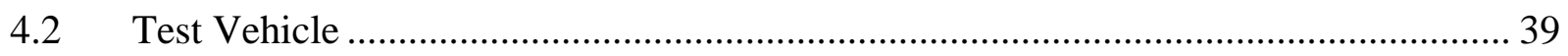

4.3 Preliminarily Extended Database................................................................................. 39

4.4 Road Load Derived Cycle Metrics ………………….................................................. 41

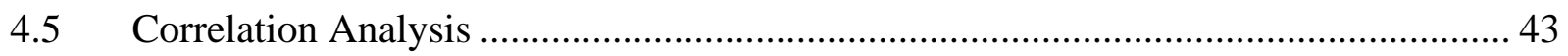

4.6 Non-parametric Correlation Analysis ....................................................................... 46

4.7 Preliminary Regression Analysis .................................................................................. 49

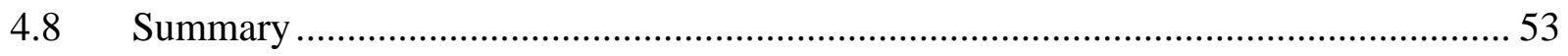

5 Generation of New Virtual Cycles with Emissions Data and Preliminary Experimental

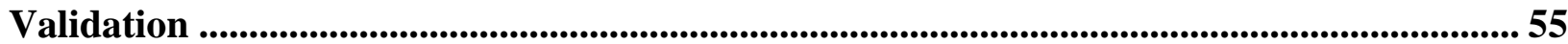

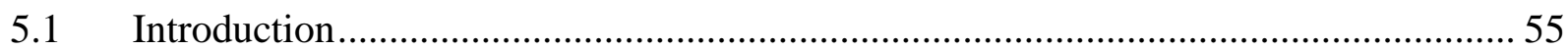

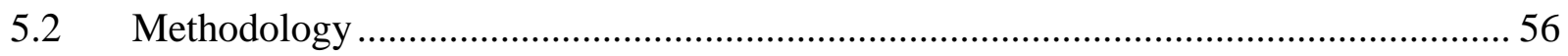

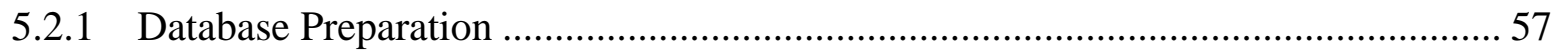

5.2.2 Description of Cycle Generator ………………................................................ 58 
5.2.3 Generation of Emissions and Fuel Economy Data ................................................... 59

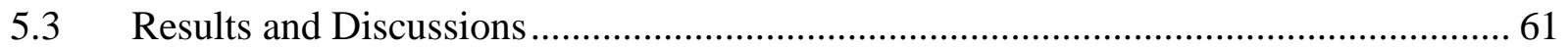

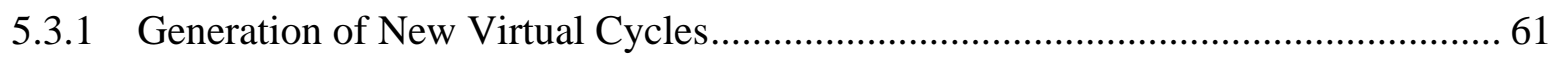

5.3.2 Generation of Emissions and Fuel Economy Data …............................................. 63

5.4 Preliminary Experimental Validation of Cycle and Emissions Generation................... 67

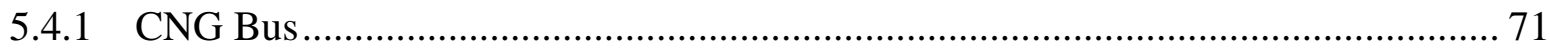

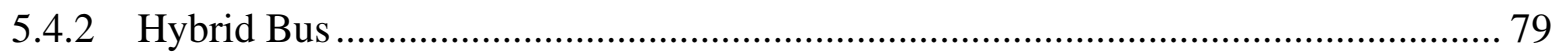

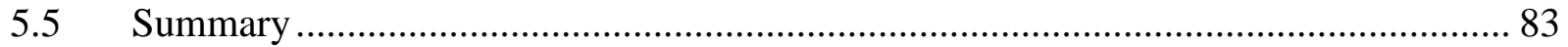

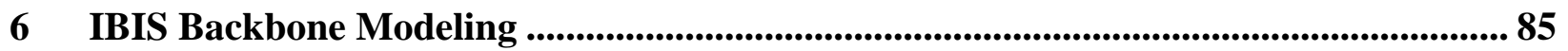

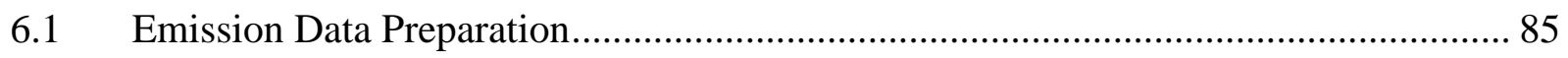

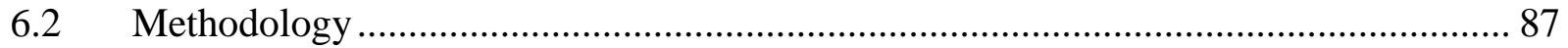

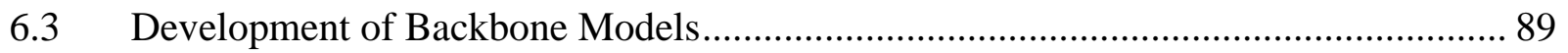

6.3.1 Models Based on the Expanded Database ................................................................. 90

6.3.2 Models Based on Tested Cycles .............................................................................. 96

6.3.3 Repair Algorithm ............................................................................................ 100

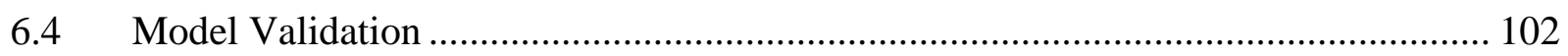

7 Development of Correction Factors .......................................................................................... 112

$7.1 \quad$ Vehicle Weight ....................................................................................................... 112

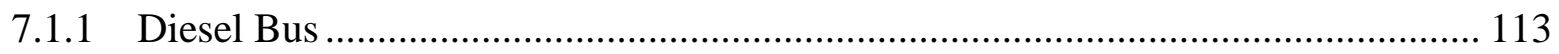

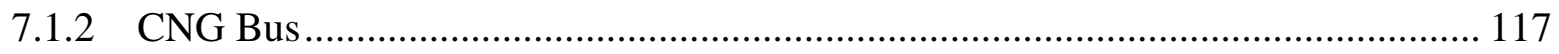

7.1.3 Road Load Equation .................................................................................... 119

7.2 Model Year Group ………………………….................................................. 124

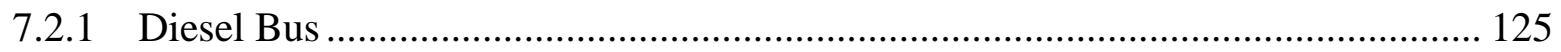

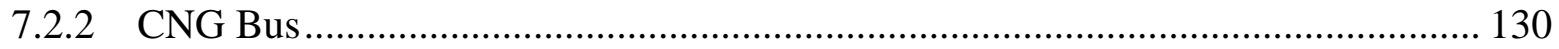

7.3 After-Treatment Technologies .......................................................................... 131

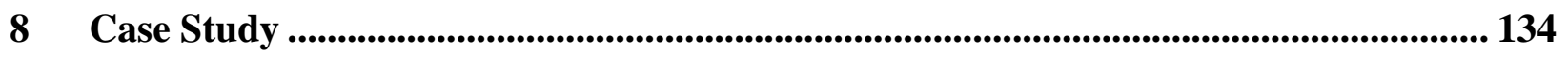

9 Conclusions and Recommendations................................................................................ 141

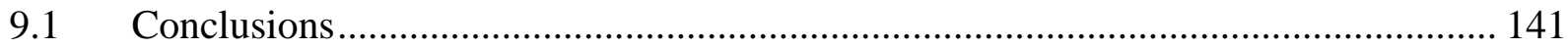

9.1.1 Correlation and Regression Study of Cycle Characteristics ................................... 141

9.1.2 Virtual Cycles, Emissions Generation and Preliminary Validation.......................... 142

9.1.3 Backbone Model Development and Validation........................................................ 143 
9.1.4 Development of Correction Factors ............................................................... 144

9.1.5 Comparison with EMFAC Model...................................................................... 145

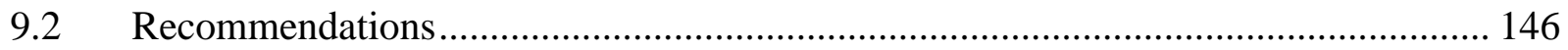

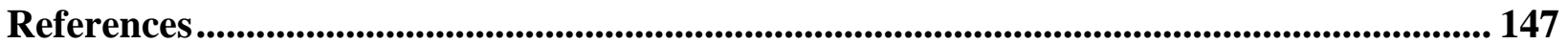

Appendix A - WVU Emission Database ............................................................................. 157

Appendix B - Emissions Testing of Virtual Cycles ........................................................ 161 


\section{LIST OF TABLES}

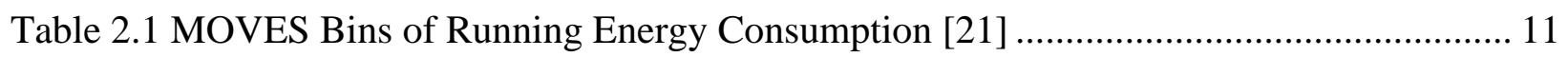

Table 2.2 MOVES Bins of Running THC, CO and NOx Emissions [21].................................. 12

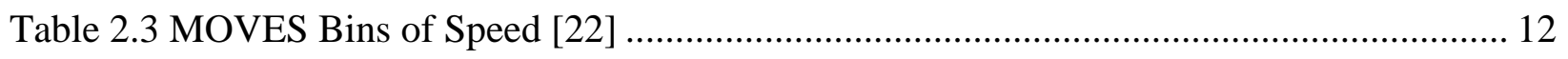

Table 2.4 Target Cycle Parameters ......................................................................................... 25

Table 4.1 Correlations of Roadload Derived Cycle Metrics with Emissions and Fuel Economy 41

Table 4.2 Correlations with Significance Levels of All Cycle Metrics .......................................... 44

Table 4.3 Correlations with Significance Levels of Four Cycle Metrics vs. Emissions and Fuel

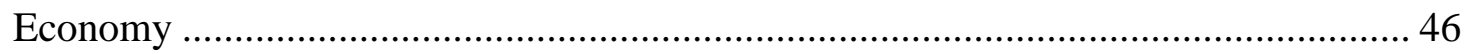

Table 4.4 Non-parametric Spearman's Correlation with Significance Levels............................... 48

Table 4.5 Summary of Order of Importance for the Selected Six Cycle Parameters .................... 49

Table 4.6 Average Speed Based Regressions ........................................................................... 51

Table 4.7 Regression Models Based on Selected Parameters......................................................... 51

Table 5.1 Cycle Statistics of the Selected Virtual Cycles................................................................ 70

Table 5.2 Results of Emissions Testing and Cycle Generation of Selected Virtual Cycles from a

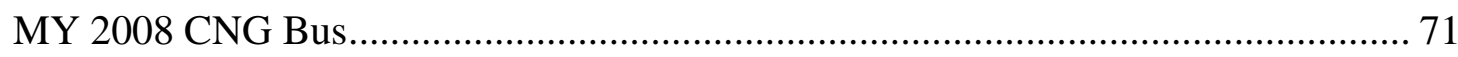

Table 5.3 Regression Results from Parity Plots of Selected Virtual Cycles from a MY 2008 CNG

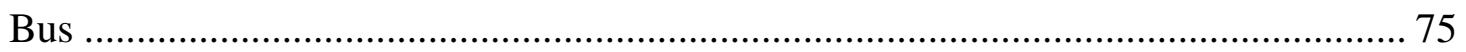

Table 5.4 Results of Emissions Testing and Cycle Generation of Selected Virtual Cycles from a MY 2008 Hybrid Bus......................................................................................... 80

Table 5.5 Regression Summary from Parity Plots of Selected Virtual Cycles from a MY 2008 Hybrid Bus ............................................................................................................... 83

Table 6.1 Vehicle Parameters of the Eight Selected Backbone Buses ............................................ 86

Table 7.1 Number of Diesel Buses Tested on CBD Cycle by Model Year Groups .................... 125

Table 7.2 Number of CNG Buses Tested on CBD Cycle by Model Year Groups...................... 130 


\section{LIST OF FIGURES}

Figure 2.1 General Architecture of the MOVES Model [20] ................................................. 11

Figure 2.2 New York Bus Cycle...................................................................................... 17

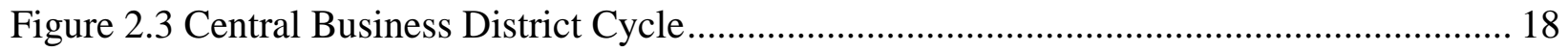

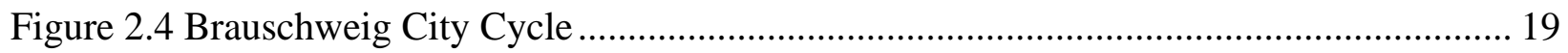

Figure 2.5 European Transient Cycle ................................................................................. 19

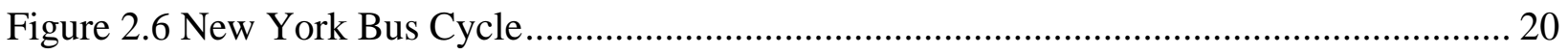

Figure 2.7 New York Composite Cycle.............................................................................. 21

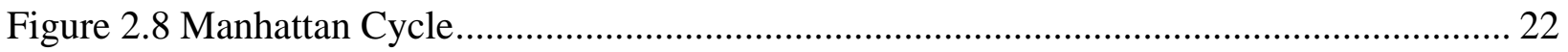

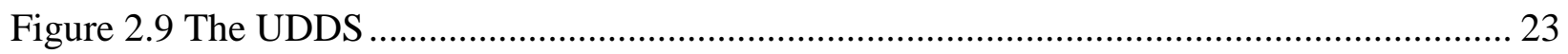

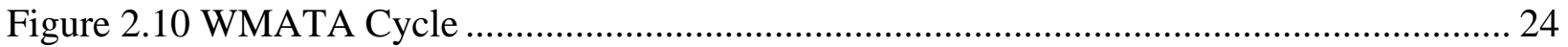

Figure 3.1 Screenshot of Preliminary Online Version of IBIS Login Page............................... 30

Figure 3.2 Screenshot of Preliminary Online Version of IBIS Emissions Model Interface......... 30

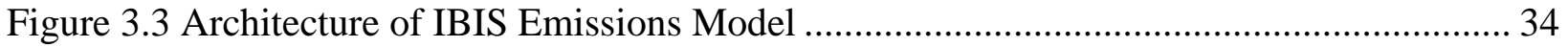

Figure 3.4 Screenshot of a Query Example from the Online Version of IBIS Searchable Bus

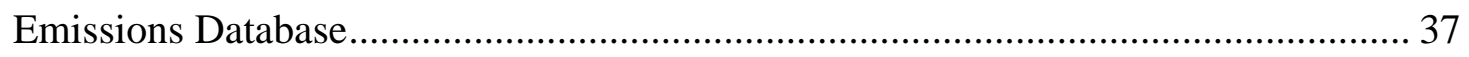

Figure 4.1 Reference Cycles and Comparison of Interpolation Curves Based on Average Speed

Figure 4.2 Comparison of Extended Dataset with Reference Cycles on the Plane of Kinetic

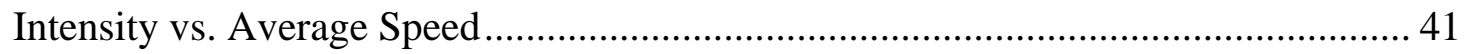

Figure 4.3 Emissions and Fuel Economy Against Kinetic Intensity ...................................... 42

Figure 4.4 $\mathrm{CO}_{2}$ Emission as a Function of Average Speed to Show Non-linear Relationship..... 47

Figure 4.5 Comparison of Single and Multiple Parameter Models to Experimental Results for the

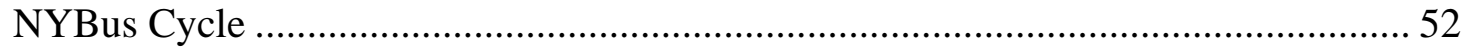

Figure 4.6 Estimation Errors of Single and Multiple Parameter Models for the NYBus Cycle... 53

Figure 4.7 Mean Percentage Error Comparison between Single and Multiple Parameter Models

Figure 5.1 Sparse Areas on Cycle Parameter Planes ........................................................... 55

Figure 5.2 The New York Bus Cycle and a Microtrip.......................................................... 57 
Figure 5.3 Flowchart for Expanding the Emissions Database for Backbone Modeling............... 60 Figure 5.4 New Virtual Cycles and Limits on Percentage Idle vs. Average Speed Plane............ 61

Figure 5.5 New Virtual Cycles and Limits on Stops/mile vs. Average Speed Plane 62

Figure 5.6 New Virtual Cycles and Limits on Standard Deviation of Vehicle Speed vs. Average Speed Plane 62

Figure 5.7 New Virtual Cycles and Limits on Kinetic Intensity vs. Average Speed Plane.......... 63

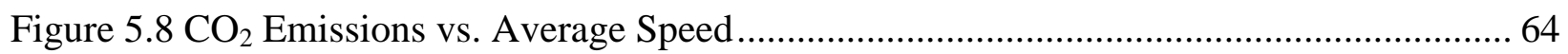

Figure 5.9 CO Emissions vs. Average Speed .................................................................... 65

Figure 5.10 HC Emissions vs. Average Speed .................................................................... 65

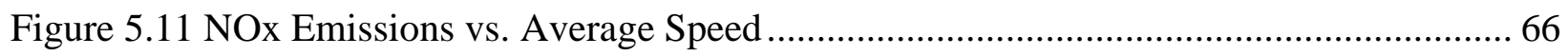

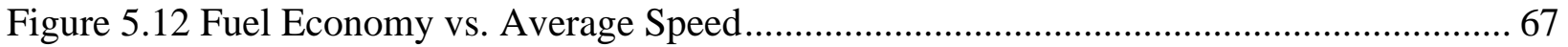

Figure 5.13 Target Speed-Time Traces of VC_274................................................................. 69

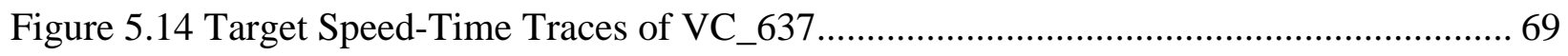

Figure 5.15 Target Speed-Time Traces of VC_28................................................................ 70

Figure 5.16 Relative Difference between Emissions Testing and Cycle Generation of Selected Virtual Cycles from a MY 2008 CNG Bus......................................................... 72

Figure 5.17 Target and Actual Vehicle Speed, Fuel Consumption and $\mathrm{CO}_{2}$ Rates of Emissions Testing and Cycle Generation of VC_637 from a MY 2008 CNG Bus ..................... 74

Figure 5.18 CO, HC and NOx Rates from Emissions Testing and Cycle Generation of VC_637 from a MY 2008 CNG Bus ......................................................................... 76

Figure 5.19 Repeatability of Vehicle Speed, Fuel Consumption and Emission Rates over the VC_637 Cycle from a MY 2008 CNG Bus .......................................................... 77

Figure 5.20 Repeatability of Vehicle Acceleration on the VC_637 Cycle from a MY 2008 CNG

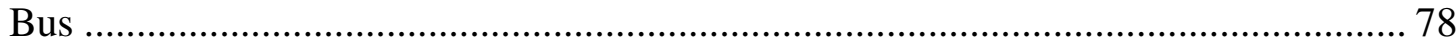

Figure 5.21 NMHC Rates from Emissions Testing and Cycle Generation of VC_637 from a MY

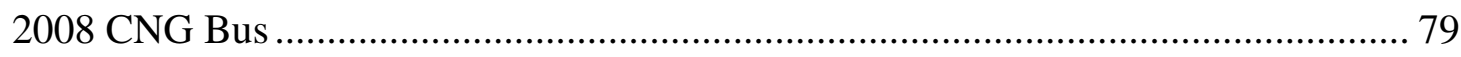

Figure 5.22 Relative Difference between Emissions Testing and Cycle Generation of Selected

Virtual Cycles from a MY 2008 Hybrid Bus ........................................................... 80

Figure 5.23 Target and Actual Vehicle Speed, Fuel Consumption and $\mathrm{CO}_{2}$ Rates from Emissions Testing and Cycle Generation of VC_274 from a MY 2008 Hybrid Bus ................... 81 
Figure 5.24 CO, HC and NOx Rates from Emissions Testing and Cycle Generation of VC_274 from a MY 2008 Hybrid Bus ............................................................................. 82

Figure 6.1 A Flowchart for General Backbone Modeling of NOx Emissions............................ 89

Figure 6.2 Fuel Economy Backbone Sub-Model Based on Average Speed and Percentage Idle

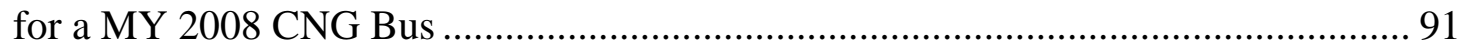

Figure 6.3 Fuel Economy Backbone Sub-Models Based on Average Speed and Other Parameters for a MY 2008 CNG Bus 93

Figure 6.4 $\mathrm{CO}_{2}$ Backbone Sub-Models in Terms of Average Speed with Percentage Idle and Kinetic Intensity Based on a MY 2008 CNG Bus ..... 93

Figure 6.5 CO Backbone Sub-Models in Terms of Average Speed with Stops/mile Based on a MY 2008 CNG Bus. 94

Figure 6.6 HC Backbone Sub-Models in Terms of Average Speed with Stops/mile Based on a MY 2008 CNG Bus 94

Figure 6.7 Selected Backbone Sub-Models Based on a MY 2008 Hybrid Bus ......................... 95

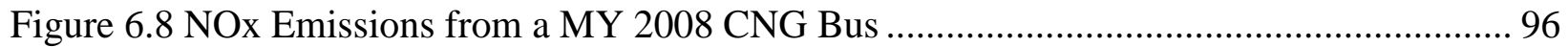

Figure 6.9 NOx Emissions from a MY 2008 CNG Bus Tested on COMM and OCTA Cycles .. 97

Figure 6.10 CO Emissions from a MY 2008 Hybrid Bus Tested on CBD and Beeline Cycles... 98 Figure 6.11 CO and HC Emissions of Tested and Generated Cycles from a MY 2008 Hybrid Bus

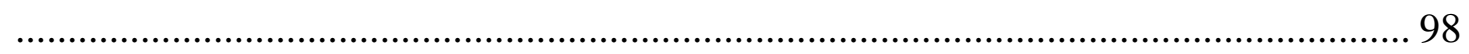

Figure 6.12 Selected NOx and PM Sub-Models of a MY 2008 CNG Bus .............................. 99

Figure 6.13 Selected CO and PM Sub-Models of a MY 2008 Hybrid Bus............................. 100

Figure 6.14 Repair Algorithm Applied to NOx Sub-Models of a MY 2008 CNG Bus ............ 101

Figure 6.15 Repair Algorithm Applied to PM Sub-Models of MY 2008 CNG and Hybrid Buses 101

Figure 6.16 Calibration and Validation Cycle Points 102

Figure 6.17 Residual Plots of Validation Cycles for the FE Sub-Models Based on Two Cycle Parameters from a MY 2008 CNG Bus 103

Figure 6.18 Residual Plots of Validation Cycles for the FE Sub-Models Based on Three Cycle Parameters from a MY 2008 CNG Bus 104

Figure 6.19 Calibration Errors of the Sub-Models Based on Two Cycle Parameters from a MY 2008 CNG Bus 105 
Figure 6.20 Validation Errors of the Sub-Models Based on Two Cycle Parameters from a MY

2008 CNG Bus 106

Figure 6.21 Validation Errors of the Combined Models Based on Three Cycle Parameters from a MY 2008 CNG Bus 107

Figure 6.22 Residual Plots of Validation Cycles for the FE Sub-Models Based on Two Cycle Parameters from a MY 2008 Hybrid Bus 108

Figure 6.23 Residual Plots of Validation Cycles for the FE Combined Models Based on Three Cycle Parameters from a MY 2008 Hybrid Bus 109

Figure 6.24 Calibration Errors of the Sub-Models Based on Two Cycle Parameters from MY 2008 Hybrid Bus . 110

Figure 6.25 Validation Errors of the Sub-Models Based on Two Cycle Parameters from MY 2008 Hybrid Bus

Figure 6.26 Validation Errors of the Combined Models Based on Three Cycle Parameters from MY 2008 Hybrid Bus. 111

Figure 7.1 Emissions and Fuel Economy of a MY 1992 Diesel Bus Retrofitted with a MY 2003

Detroit Diesel Engine Tested at Different Weights ..... 115

Figure 7.2 The Ratios of Emissions and Fuel Economy of a MY 1992 Diesel Bus Retrofitted with a MY 2003 Detroit Diesel Engine Tested at Different Weights .... 116

Figure 7.3 The Average Ratios of Emissions and Fuel Economy Based on Microtrip Data from a MY 1992 Diesel Bus Retrofitted with a MY 2003 Detroit Diesel Engine Tested at Different Weights.

Figure 7.4 The Ratios of Emissions and Fuel Economy of a MY 2005 CNG Bus with a John

Deere Engine Tested at Different Weights 118

Figure 7.5 The Average Ratios of Emissions and Fuel Economy Based on Microtrip Data from a

MY 2005 CNG Bus with a John Deere Engine Tested at Different Weights 119

Figure 7.6 Weight Correction Factors for Fuel Economy as a Function of Average Speed for the

Diesel Backbone Model of MY group 1998-2002. 124

Figure 7.7 Weight Correction Factors for Emissions as a Function of Average Speed for the

Diesel Backbone Model of MY group 1998-2002. 124

Figure 7.8 Average CBD Cycle Ratios from Other MY Groups Compared to MY 1998 - 2002

Diesel Backbone Model 126 
Figure 7.9 Emissions Testing Data of Diesel Buses from MY 1994 - 1997 and Adjusted Values

Based on CBD Ratios from the Diesel Backbone Bus of MY 1998 - 2002 127

Figure 7.10 Average NYBus and WMATA Cycle Ratios from MY 1994 - 1997 Compared to MY 1998 - 2002 Diesel Backbone Model 128

Figure 7.11 Emissions and Fuel Economy Ratios of MY 1998 - 2002 to MY 2003 - 2006 Diesel

Backbone Bus as a Function of Average Speed 129

Figure 7.12 Average CBD Cycle Ratios from Other MY Groups Compared to MY 2003 - 2006

CNG Backbone Model 131

Figure 7.13 Average Emissions and Fuel Economy Reduction Percentage of Diesel Buses with After-treatment Technologies 133

Figure 8.1 Comparisons of the EMFAC and IBIS Models for MY 2000 Diesel Buses in Calendar Year 2005 135

Figure 8.2 Comparisons of the EMFAC and IBIS Models for MY 2006 Diesel Buses in Calendar Year 2006 137

Figure 8.3 Comparisons of the EMFAC and IBIS Models for MY 2000 Diesel Buses from 2000 to 2010 139

Figure 8.4 The EMFAC Model for Diesel Buses from MY 1992 to 2012 140 


\section{NOMENCLATURE}

\begin{tabular}{ll} 
ADVISOR & ADvanced VehIcle SimulatOR \\
AeroV & Aerodynamic Speed \\
ART & Arterial Cycle \\
AspedWID & Average Vehicle Speed with Idle \\
AspedWoID & Average Vehicle Speed Without Idle \\
AveAcc & Average Acceleration \\
AveDec & Average Deceleration \\
ANN & Artificial Neural Network \\
BEELINE & Westchester County NY Beeline Cycle \\
BRAUN & Braunschweig Cycle \\
CAFEE & Center for Alternative Fuels, Engines, and Emissions \\
CARB & California Air Resources Board \\
CBD & Central Business District Cycle \\
CE-CERT & Center for Environmental Research and Technology \\
CF & Correcton Factor \\
CFR & Code of Federal Regulations \\
CharAcc & Characteristic Acceleration \\
CMEM & Comprehensive Modal Emission Model \\
CNG & Compressed Natural Gas \\
CO & Carbon Monoxide \\
CO & Carbon Dioxide \\
COMM & Commuter Cycle \\
CV & Coefficient of Variance \\
\hline
\end{tabular}




\begin{tabular}{|c|c|}
\hline D1 & Diesel \# 1 \\
\hline D2 & Diesel \# 2 \\
\hline DOC & Diesel Oxidation Catalyst \\
\hline DOT & Department of Transportation \\
\hline DPF & Diesel Particulate Filter \\
\hline EF & Emission Factor \\
\hline EGR & Exhaust Gas Recirculation \\
\hline EMFAC & EMission FACtors Model \\
\hline EPA & Environmental Protection Agency \\
\hline ETC & European Transient Cycle \\
\hline ETC_1 & European Transient Cycle - Urban \\
\hline FTA & Federal Transit Administration \\
\hline FTP & Federal Test Procedure \\
\hline FuelEco & Fuel Economy \\
\hline GUI & Graphical User Interface \\
\hline GVW & Gross Vehicle Weight \\
\hline GVWR & Gross Vehicle Weight Rating \\
\hline $\mathrm{HC}$ & Hydrocarbon \\
\hline HDD & Heavy-Duty Diesel Vehicle \\
\hline HDV & Heavy-Duty Vehicle \\
\hline HFID & Heated Flame Ionization Detector \\
\hline HTBR & Hierachical Tree Based Regression \\
\hline IBIS & Integrated Bus Information System \\
\hline KInt & Kinetic Intensity \\
\hline LA & Los Angeles \\
\hline
\end{tabular}




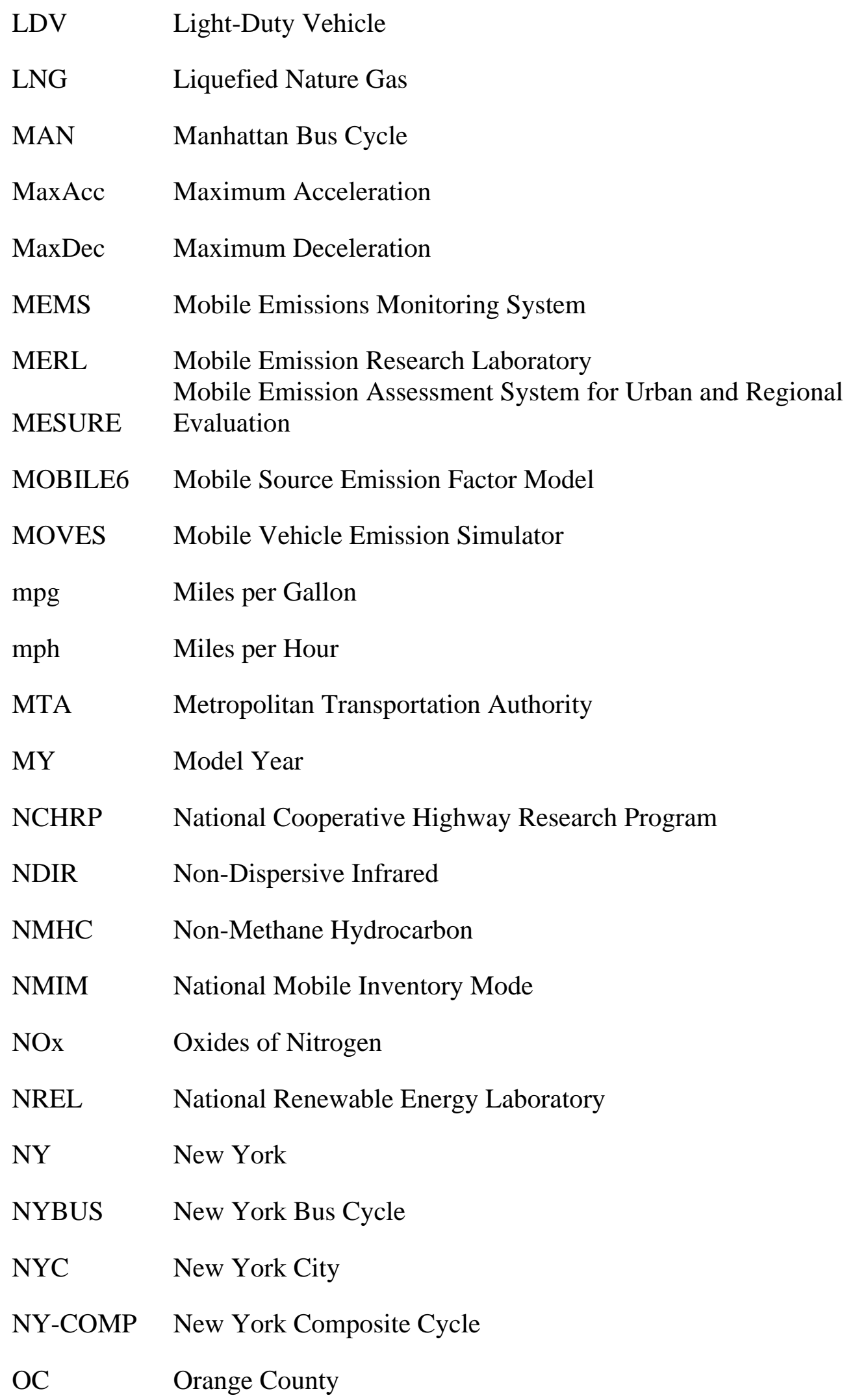




\begin{tabular}{|c|c|}
\hline OCTA & Orange County Transit Authority Cycle \\
\hline OLS & Ordinary Least Squares \\
\hline PercID & Percentage Idle \\
\hline PM & Particulate Matter \\
\hline ppm & Parts per Million \\
\hline RMS & Root Mean Square \\
\hline RMSE & Root Mean Square Error \\
\hline ROG & Reactive Organic Gases \\
\hline SAE & Society of Automotive Engineers \\
\hline $\mathrm{SO}_{2}$ & Sulfur Dioxide \\
\hline SOC & State of Charge \\
\hline Stops/Mi & Stops per Mile \\
\hline THC & Total Hydrocarbons \\
\hline TOG & Total Organic Gases \\
\hline TransLab & Transportable Heavy-Duty Vehicle Emission Laboratory \\
\hline TWC & Three-Way Catalyst \\
\hline UDDS & Urban Dynamometer Driving Schedule \\
\hline ULSD1 & Ultra Low Sulfur Diesel \#1 \\
\hline VC & Virtual Cycle \\
\hline VC_274 & Virtual Cycle \#274 \\
\hline VC_28 & Virtual Cycle \#28 \\
\hline VC_637 & Virtual Cycle \#637 \\
\hline VMT & Vehicle Miles Traveled \\
\hline VMY & Vehicle Model Year \\
\hline VSP & Vehicle Specific Power \\
\hline
\end{tabular}


VstdWID Standard Deviation of Vehicle Speed with Idle

VstdWoID Standard Deviation of Vehicle Speed without Idle

WMATA Washington Metropolitan Area Transit Authority

WVU West Virginia University 


\section{INTRODUCTION}

\subsection{Problem Definition}

Heavy-duty vehicles are known to contribute significant pollution to the atmospheric inventory and emission standards have become tighter with increasing model year. As a result, many transit agencies strive to reduce their environmental impact by retrofitting existing buses or replacing old higher emitting buses. Emissions testing for transit buses requires high cost and extensive effort is involved.

In order to characterize the emissions and fuel consumption from vehicles, numerous predictive emissions models have been developed as functions of influential parameters. Based on input characteristics, emission models can be divided into two categories including microscopic and macroscopic models. Microscopic models are based on instantaneous data and provide more accurate estimations compared with macroscopic ones. However, those models have penalties in that much longer time is needed for data processing, and extensive professional skills are required to utilize the models due to their complicated natures. On the other hand, macroscopic models employ integrated data and are much simpler and faster but with compromised accuracy. It becomes necessary and urgent to develop a novel predictive system to assist transit agencies or government in evaluating the emissions implications of new transit vehicle procurements.

\subsection{Research Purpose}

The overall purpose of this dissertation was to develop the Integrated Bus Information System (IBIS) for the Federal Transit Administration (FTA) to evaluate emission inventories and fuel economy (mpg) for transit buses, providing current transit agencies useful information for evaluating new vehicles they plan to purchase. Compared with existing models, the emissions prediction model was intended to be less complicated to use but have sufficient accuracy to estimate and predict emissions and fuel economy implications of different transit bus fuel and propulsion system options. Fuel economy (mpg) and distance specific emissions (g/mile) were evaluated and predicted in this study, including carbon monoxide (CO), carbon dioxide $\left(\mathrm{CO}_{2}\right)$, 
oxides of nitrogen (NOx), hydrocarbons (HC), non-methane hydrocarbons (NMHC) (for CNG buses) and particulate matter (PM).

\subsection{Research Objectives and Approaches}

To achieve the overall purpose of this study, specific sub-tasks and approaches were established.

Task 1. To initially determine the overall strategies and methodologies to develop the IBIS transit fleet emissions model, a literature review of existing emission modeling approaches was conducted.

- Study existing emission models and understand their advantages and limitations.

- Review related publications, reports and documents to investigate possible parameters affecting emissions and fuel economy.

- Familiar with WVU databases, including continuous data, integrated data, and short report data from various transit buses with the main focuses on diesel, CNG and hybrid vehicles.

- Study existing emission modeling software or tools with their inputs, outputs and interfaces and preliminarily determine the general structure of the IBIS model.

Task 2. To determine the most influential characteristics of driving cycles that influence emissions and fuel economy, correlation and regression analyses were conducted and the cycle parameters to be used for the IBIS inputs were selected.

- Conduct a literature review on the impact of existing standard chassis dynamometer cycles on emissions.

- Explore cycle parameters that reflected the characteristics of chassis dynamometer cycles and write a computer program to compute them.

- Choose a representative transit bus tested on multiple cycles and apply appropriate interpolation to initially expand the emissions database for the purpose of statistical analysis.

- Conduct parametric and non-parametric statistical correlation analysis among cycle parameters and between cycle parameters and emissions, then consequently determine the most influential cycle parameters for modeling. 
- Perform a preliminary regression analysis to demonstrate potential model improvement by including extra cycle parameters beyond average speed, indicating further modeling strategies.

Task 3. To expand coverage of the parameter space of emissions data for modeling purposes, virtual cycles were developed from existing data to fill sparse areas of the parameter space.

- Conduct a literature review of existing cycle/schedule generation methodologies then determine the general strategies to develop the cycle generator.

- Define and generate microtrips and prepare databases for cycle generation.

- Collaborate with other team members to design and implement the computational algorithm.

- Generate numerous virtual cycle points to cover the sparse areas of cycle parameter planes spanned by average speed with each of the other influential cycle parameters selected in Task 2.

- Select virtual cycles for emissions testing and use the results to experimentally validate the cycle generation method.

Task 4. In collaboration with other team members, IBIS backbone emissions predictive models for diesel, hybrid, and CNG buses were developed.

- Analyze and process emissions data of the selected representative transit buses (backbone buses) tested on multiple standard chassis cycles for emissions database preparation.

- Write a computer program to produce continuous emissions and fuel consumption data for each backbone bus over a full set of virtual cycles generated in Task 3 and to integrate data produced as distance-specific emissions data for modeling purposes.

- Collaborate with other team members to develop the computation tool for backbone modeling.

- Use the computation tool to build backbone models for newer (model year 2008) transit buses.

- Validate backbone models using two and three cycle parameters based on additional virtual cycle and standard cycle data. 
Task 5. Correction factors accounting for the impact of non-driving characteristics were developed.

- Investigate the weight impact through studying road load equation and conducting data analysis using the microtrip method based on transit buses tested at different passenger loads.

- Perform data analysis for diesel and CNG buses and seek a practical method to determine correction factors due to model year variations.

- Determine correction factors of after-treatment technologies for diesel buses.

Task 6. To provide a case study to compare IBIS emission model with CARB's EMFAC model.

\subsection{Anticipated Contributions}

The primary contribution of this dissertation is to provide government and transit agencies a simple and accurate predictive emissions model for evaluating emissions and fuel economy implications of new transit vehicle procurement. In addition to the primary contribution, the following major contributions also resulted from this study.

A comprehensive correlation analysis of driving cycle characteristics with emissions and fuel economy identified possible influential driving characteristic for emissions and fuel economy modeling and provided a useful framework for the selection of the most influential driving cycle parameters.

Generation of virtual cycles from existing test data demonstrated a useful method to improve the database for emission modeling and thus reduce expensive emissions testing. In addition, the preliminary experimental validation of the cycle generation method provided the insight on whether relative positions or histories of microtrips (or segments) could affect the emissions and fuel consumption rates as well as their integrated values over a cycle.

\subsection{Dissertation Layout}

This dissertation consists of nine chapters. It began with literature reviews in Chapter 2 . In Chapter 3, the inputs, outputs, and general structure of IBIS transit fleet emissions modeling were introduced as well as the emissions testing facilities and emissions database employed in this research. In Chapter 4, correlation and regression analyses were performed to investigate the 
impact of driving cycle characteristics on emissions. The importance of specific cycle parameters was analyzed and the most influential parameters were determined as explanatory variables of driving characteristics for the IBIS modeling. Chapter 5 introduced a novel framework to generate new virtual cycles associated with their emissions data from the limited number of chassis dynamometer data available to expand the emissions database used for the development of the IBIS model. In addition, the cycle generation method was validated from emissions testing on selected virtual cycles. Chapter 6 described strategies for backbone modeling. Backbone models for transit buses from recent model years was developed and validated. Chapter 7 analyzed the impacts of non-driving parameters such as vehicle weight, model years, and aftertreatment technologies. Consequently, corresponding correction factors for each parameter were determined. Finally, a case study was provided to compare IBIS emissions model with CARB's EMFAC. The key findings from previous chapters as well as recommendations for future research were summarized in the last chapter. 


\section{LITERATURE REVIEW}

The concern over emissions from transit buses is rising since they contribute significantly to air pollution in urban areas. Additionally, fuel consumption is another concern, considering increasing fuel prices.

\subsection{Characterization of Emissions and Fuel Economy}

There are three major methodologies for measuring emissions and fuel economy, including laboratory-based dynamometer tests, remote sensing tests, and on-board measuring systems. The laboratory-based dynamometer test includes engine and chassis dynamometer tests. Both kinds of tests acquire emission data by performing specific test schedules or cycles.

Engine dynamometer testing requires the engine to be removed from the vehicle, thus extra labor is required and emission data do not account for vehicle specifications and conditions. The advantage of the test is high repeatability. On the other hand, a chassis dynamometer test can reflect the conditions of both engine and vehicle. However, emission data from both types of laboratory-based dynamometer tests might not be representative of actual on-road vehicle operations [1, 2]. Another finding from the Transit Cooperative Research Program (TCRP) C-15 project conducted by West Virginia University (WVU), suggested that on-road fuel economy is lower than the data from chassis dynamometer tests for diesel buses [3]. The discrepancy might be due to different weather and terrain effects or the impact of heating and air conditioning since chassis dynamometer tests usually do not account for these.

Remote sensing devices use infrared or ultraviolet spectroscopy to measure the exhaust emissions when a vehicle passes a sensor on the road. Remote sensing is primarily used to evaluate the effectiveness of inspection and maintenance programs, detect high emitting vehicles, and develop emission factors [1]. The extensive use of remote sensing devices could provide real-time emissions of on-road vehicles with better spatial and temporal distributions [4]. The advantage of the remote sensing method is that it can measure emissions from large quantities of on-road vehicles. The disadvantages include the limitations from test locations and weather conditions [1]. 
On-board emission measuring systems were developed to measure the on-road emissions from actual vehicle operation. On-board measuring systems provide the instantaneous data of vehicle activities and emissions, making the on-road data highly temporal and spatial. The main reason that on-board measuring systems are not widely employed is attributed to their high cost [1]. Another reason might be that the various on-road conditions affect the measurement accuracy of the test equipment. For example, the characteristics of particulate matter (PM) require a stable environment for accurate measurement. On this point, laboratory-based dynamometer tests are much better. Additionally, on-board emission tests are generally not repeatable, considering high variations from drivers and various road traffic conditions.

\subsection{Emissions Models}

In order to characterize and predict the emissions and fuel consumption from vehicles, based on emission test data, many emission inventory models have been developed as functions of influential parameters. These parameters include vehicle parameters, vehicle operation parameters, fuel type, engine specifications, road conditions, and ambient conditions. These parameters can be continuous, discrete, or categorical. Based on the characteristics of the input parameters, emission models can be classified into two categories. One category is microscopic or microscale models based on instantaneous inputs such as second-by-second engine speed or power, vehicle speed and acceleration. The outputs of this category of models usually include instantaneous emission rates (g/s) and fuel consumption (gal/s). The other category is macroscopic or macroscale models based on inputs with integrated statistic characteristics or average values of velocity profile over time or distance traveled. The outputs of this category of

models can be distance, time or energy specific emission values and averaged fuel economy (mpg). Other categorical parameters such as model year (MY), fuel type, after-treatment technologies, etc., can be considered as correction factors in both categories of models due to their significant impact on emissions and fuel economy. A brief description of existing emission models is provided below. 


\subsubsection{Macroscopic Models}

\subsubsection{MOBILE Model and EMFAC Model}

One well-known inventory model in the macroscopic category was the Mobile Source Emission Factor Model (MOBILE6) developed by the US Environmental Protection Agency (EPA) [5]. The software was written in Fortran and the latest version of MOBILE6 was MOBILE6.2, which provided emission factors in grams per mile from on-road vehicles, including $\mathrm{CO}, \mathrm{NOx}, \mathrm{HC}, \mathrm{CO}_{2}, \mathrm{PM}$, sulfur dioxide $\left(\mathrm{SO}_{2}\right)$, etc. The general structure of the MOBILE model can be expressed as the following equation [6]:

$$
E F=\sum_{m=1}^{n} V M T_{m} *(B E R * C F s)
$$

Equation 2.1

where $E F$ refers to the emission factor, $V M T_{m}$ refers to the fraction of vehicle miles traveled in MY $m, n$ is the total number of the MYs, $B E R$ is base emission rates, and $C F \mathrm{~s}$ represent correction factors [6].

MOBILE was able to calculate basic emission rates for each vehicle class based on standard driving cycles and operational conditions. The basic emission rates were corrected by numerous factors including speed, air conditioning, fuel characteristics, deterioration, and ambient temperatures, etc. [5]. For light-duty vehicles, the basic emission rates were determined from the Federal Test Procedure (FTP) [7]. Emissions rates at average speeds other than that of the FTP cycle were corrected by speed correction factors. For heavy-duty vehicles (HDVs), the basic emission rates were obtained from engine dynamometer tests. To obtain distance specific emission factors in gram per mile, a conversion factor must be used. The speed correction factors for HDVs were developed based on 22 vintage heavy-duty diesel trucks tested on the chassis dynamometer [7].

The limitation of the MOBILE model was that only average speed was employed to reflect driving characteristics. It was widely accepted that various driving profiles could result in approximately the same average speed while dramatically different emissions were produced.

MOBILE 6.2 has 27 input parameters such as calendar year, altitude, fuel characteristics (sulfur content, oxygenate content, etc), registration distribution by vehicle class, annual mileage accumulation by vehicle class, and average speed distribution by vehicle class, etc. [5]. The users 
are not required to input values for most parameters except calendar year, minimum and maximum daily temperature, and fuel volatility.

Due to the complexity of the user interface and the inherent assumptions regarding fleet demographics and vehicle duty cycles, MOBILE6 is not well suited for use as an analysis tool for transit vehicle procurement.

The EMission FACtors (EMFAC) model was a well-known inventory model developed by the California Air Resources Board (CARB) [8-10]. The principle of the EMFAC model was similar to that of the EPA's MOBILE and it estimated emission factors as a product of basic emission rates and vehicle activities [7]. It suffered the same drawback as MOBILE in that the basic emission rates were based on average speed of tested driving cycles, thus the model was limited by narrow profiles of driving cycle characteristics.

EMFAC2011 was the latest version of the model $[9,10]$ and it provided web based data access. It includes three modules: EMFAC2011-LDV, EMFAC2011-HD, and EMFAC2011-SG. EMFAC2011-LDV module provides emissions estimation for gasoline vehicles, diesel vehicles with a gross vehicle weight rating (GVWR) below 14,000 lbs and urban transit buses while EMFAC2011-HD module estimates emissions for diesel vehicles with a GVWR above 14,000 lbs. EMFAC2011-SG combines the outputs from the first two modules and applies scaling factors to provide scenario analysis for transportation planning.

\subsubsection{Other Macroscopic Models}

Other macroscopic models include the NONROAD [11] and the National Mobile Inventory Model (NMIM) [12] developed by the EPA. NONROAD was used to predict emissions from non-road vehicles and equipment. NMIM combined both MOBILE and NONROAD and estimates emissions from both on- and off-road vehicles and equipment. Similarly, for non-road vehicles, CARB developed the OFFROAD model [13] to estimate the emissions from off-road mobile sources. Since the principles of these emission models were similar to those of the MOBILE, the limitations from the MOBILE also applied to these models.

\subsubsection{Microscopic Models}

Microscopic models used continuous data to account for the transient nature of various driving activities which significantly affect the emission rates and fuel consumption. 


\subsubsection{MOVES}

Considering the drawback from the MOBILE, NONROAD, and NMIM models, EPA developed a mobile emission model, entitled the MOtor Vehicle Emission Simulator (MOVES) [14-18] to replace the existing inventory models. The model has been continually improved and multiple versions have been released since 2004: MOVES2004 [14], MOVES2009 [15], MOVES2010 [16], MOVES2010a [17] and recently released MOVES 2010b [18]. The principle of MOVES firstly characterized vehicle activity then investigated the relationships among vehicle activity, energy consumption, and emissions [19]. Unlike the NMIM model that physically incorporated MOBILE and NONROAD, MOVES implemented a binning method and used second-by-second data to develop emission rates. MOVES could provide multi-level analysis including macroscale analysis (national level using counties as spatial units), mesoscale analysis (regional level using route links as spatial units), and microscale analysis (project level using specific points such as a transportation passageway or intersection) [20].

Figure 2.1 illustrates the general architecture of the MOVES [20]. The major processes of the MOVES are: total activity is distributed into source and operating mode bins; then emission rates are retrieved for source and operating mode bins; total emissions are calculated for different processes based on emission rates and bin distribution information [20].

Regarding total activity, different emission process use different measures. For example, source-time is used for most of emission processes such as running exhaust while number of starts is used for start exhaust. Source bins are characterized by multiple subcategory parameters such as fuel type, vehicle weight, model year group, engine size, etc.

Unlike the MOBILE model which used average speed to reflect operational characteristics, one of the main characteristics of MOVES was that it employed the instantaneous speed (IS) and vehicle specific power (VSP) to characterize the operation modes. VSP accounts for speed, acceleration, grade, and road load. Energy consumption and emissions rates were binned according to IS and VSP as shown in Table 2.1 [21] and Table 2.2 [21], respectively. Combination of IS and VSP provided more information about transient operation characteristics that significantly affect emissions and fuel economy. To be noted, different operating model parameters were applied to different emissions processes [20]. For example, average speed or VSP were used for running exhaust while soak time was used for start exhaust. 


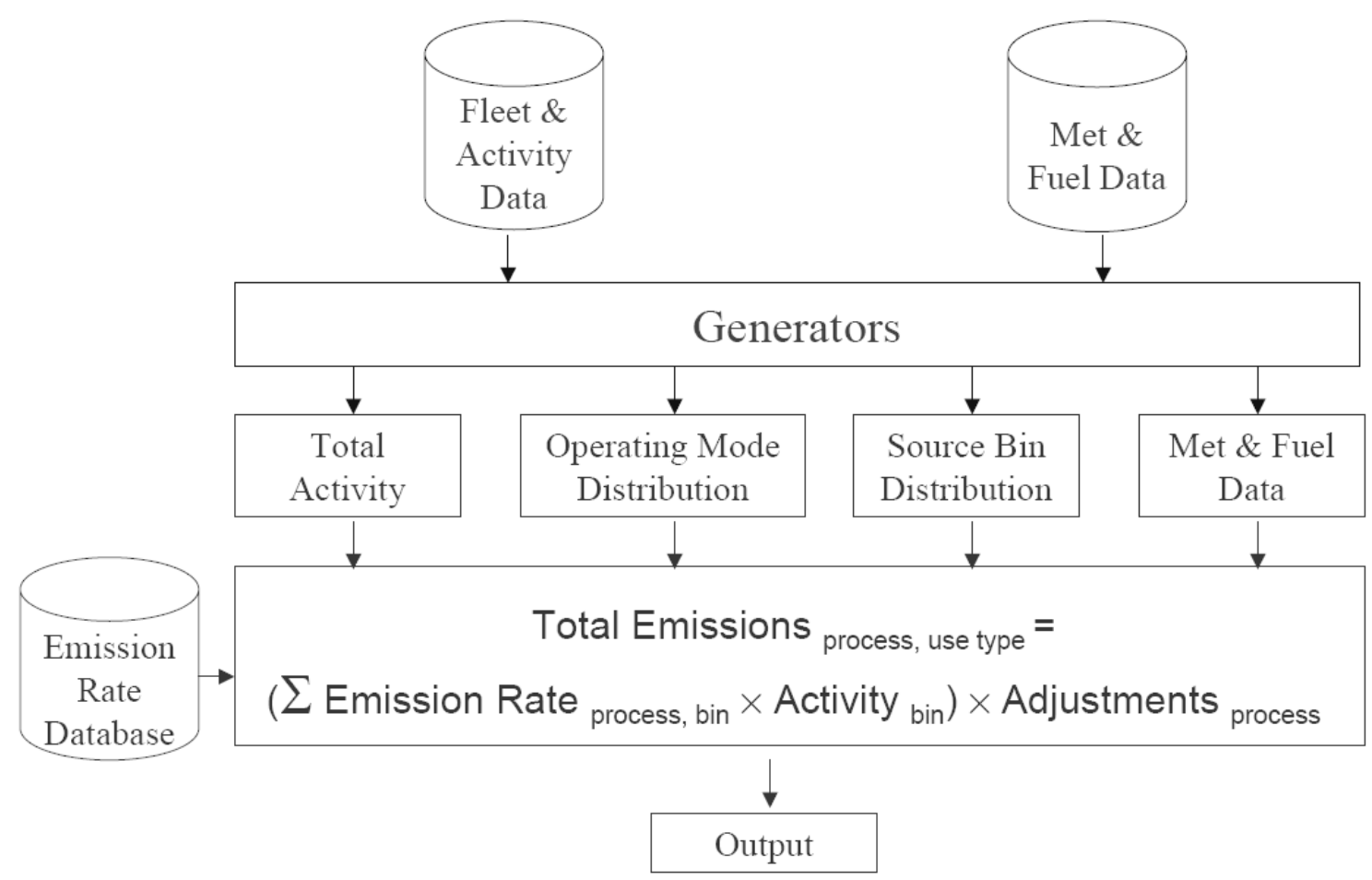

Figure 2.1 General Architecture of the MOVES Model [20]

Table 2.1 MOVES Bins of Running Energy Consumption [21]

\begin{tabular}{llll}
\hline Braking (Bin 0) & & & \\
\hline Idle (Bin 1) & & & \\
\hline VSP $\backslash$ Instantaneous Speed & $\mathbf{0 - 2 5 m p h}$ & $\mathbf{2 5 - 5 0}$ & $>\mathbf{5 0}$ \\
\hline$<0 \mathrm{~kW} /$ tonne & Bin 11 & Bin 21 & - \\
0 to 3 & Bin 12 & Bin 22 & - \\
3 to 6 & Bin 13 & Bin 23 & - \\
6 to 9 & Bin 14 & Bin 24 & - \\
9 to 12 & Bin 15 & Bin 25 & - \\
12 and greater & Bin 16 & Bin 26 & Bin 36 \\
6 to 12 & - & - & Bin 35 \\
$<6$ & - & - & Bin 33 \\
\hline
\end{tabular}

Users could not input average speed directly. Average speed is needed to convert to speed distribution as shown in Table 2.3 [22]. If average speed was not equal to one of the average bin speed, then a linear interpolation could be used to determine the Bin's fraction from its adjacent speed bins. It was suggested by the EPA to use detailed speed information to determine speed distribution rather than a single average speed [22]. 
Table 2.2 MOVES Bins of Running THC, CO and NOx Emissions [21]

\begin{tabular}{llll}
\hline Braking (Bin 0) & & & \\
\hline Idle (Bin 1) & & & \\
\hline VSP $\backslash$ Instantaneous Speed & $\mathbf{0 - 2 5 m p h}$ & $\mathbf{2 5 - 5 0}$ & $>\mathbf{5 0}$ \\
\hline$<0 \mathrm{~kW} /$ tonne & Bin 11 & Bin 21 & - \\
0 to 3 & Bin 12 & Bin 22 & - \\
3 to 6 & Bin 13 & Bin 23 & - \\
6 to 9 & Bin 14 & Bin 24 & - \\
9 to 12 & Bin 15 & Bin 25 & - \\
12 and greater & Bin 16 & Bin 26 & - \\
12 to 18 & - & Bin 27 & Bin 37 \\
18 to 24 & - & Bin 28 & Bin 38 \\
24 to 30 & - & Bin 29 & Bin 39 \\
30 and greater & - & Bin 30 & Bin 40 \\
6 to 12 & - & - & Bin 35 \\
$<6$ & - & - & Bin 33 \\
\hline
\end{tabular}

Table 2.3 MOVES Bins of Speed [22]

\begin{tabular}{rrl}
\hline \multicolumn{1}{l}{ Speed Bin ID } & Average Bin Speed & Speed Bin Range \\
\hline 1 & 2.5 & speed $<2.5 \mathrm{mph}$ \\
2 & 5 & $2.5 \mathrm{mph}<=$ speed $<7.5 \mathrm{mph}$ \\
3 & 10 & $7.5 \mathrm{mph}<=$ speed $<12.5 \mathrm{mph}$ \\
4 & 15 & $12.5 \mathrm{mph}<=$ speed $<17.5 \mathrm{mph}$ \\
5 & 20 & $17.5 \mathrm{mph}<=$ speed $<22.5 \mathrm{mph}$ \\
6 & 25 & $22.5 \mathrm{mph}<=$ speed $<27.5 \mathrm{mph}$ \\
7 & 30 & $27.5 \mathrm{mph}<=$ speed $<32.5 \mathrm{mph}$ \\
8 & 35 & $32.5 \mathrm{mph}<=$ speed $<37.5 \mathrm{mph}$ \\
9 & 40 & $37.5 \mathrm{mph}<=$ speed $<42.5 \mathrm{mph}$ \\
10 & 45 & $42.5 \mathrm{mph}<=$ speed $<47.5 \mathrm{mph}$ \\
11 & 50 & $47.5 \mathrm{mph}<=$ speed $<52.5 \mathrm{mph}$ \\
12 & 55 & $52.5 \mathrm{mph}<=$ speed $<57.5 \mathrm{mph}$ \\
13 & 60 & $57.5 \mathrm{mph}<=$ speed $<62.5 \mathrm{mph}$ \\
14 & 65 & $62.5 \mathrm{mph}<=$ speed $<67.5 \mathrm{mph}$ \\
15 & 70 & $67.5 \mathrm{mph}<=$ speed $<72.5 \mathrm{mph}$ \\
16 & 75 & $72.5 \mathrm{mph}<=$ speed \\
\hline
\end{tabular}

MOVES was written in JAVA and MySQL [23]. MOVES is a complex model with multiple MySQL databases, such as default input database, optional user input databases, MOVESExecution database, output database, and MOVESWorker database. MOVES usually requires the user to create input and output databases to run the model [18]. Since MOVES outputs are MySQL databases, if users need to view the results from Microsoft Excel, a setup is needed to connect the MySQL tables. In addition, compared to the MOBILE6.2, MOVES requires significantly longer running time for a similar task. For example, if a single desktop 
computer was used, MOVES2004 could use one hour to finish a 20-second run performed by the MOBILE6.2 [24].

\subsubsection{Modal Binning Method}

Another "binning” approach was developed by North Carolina State University (NCSU) and called a modal binning method. The conceptual differences between the NCSU method and the VSP based EPA method lies in how the operating modes were defined [25]. NCSU defined the vehicle operation bins based on speed, acceleration, and power demand, and divided the data into four modes (cruise, acceleration, deceleration, and idle). A modeling database consisted of approximately 232,000 seconds of data from both laboratory dynamometer and on-board emission tests [26]. Within each modal bin, the explanatory variables were selected by hierarchical tree based regression (HTBR) then ordinary least squares (OLS) regression analysis was performed to develop the emission models [25, 27]. The explanatory variables included vehicle speed, acceleration, ambient temperature, humidity, altitude, road grade, engine size, power demand, and the second and third powers of vehicle speed and acceleration. However, many terms were proved insignificant and were removed for different modes [25].

\subsubsection{MEASURE}

The Mobile Emission Assessment System for Urban and Regional Evaluation (MEASURE) model was developed by Georgia Institute of Technology. The principle of the MEASURE model was similar to that of the modal binning method from NCSU. The model estimated emissions based on different operation modes including cruise, acceleration, deceleration, idle and power demand conditions [28]. Vehicle operation modes were defined based on average speed, roadway characteristics, traffic flow and volume to capacity ratio. The database consisted of more than 13,000 laboratory tests conducted by the CARB and EPA using standardized and alternative test cycles [28-30]. The HTBR was employed to reduce the number of explanatory variables and to identify useful interactions among the predictors. Then OLS regression was used to build the emission models as a function of modal activity parameters and vehicle technologies. Model activity parameters included average speed, acceleration, deceleration, cruise, idle, inertial power surrogate (defined as acceleration times velocity), and 
drag power surrogate (defined as acceleration times velocity squared). Technology related variables included fuel injection and catalytic converter [29].

\subsubsection{Speed-Acceleration Method}

Compared to the IS and VSP based bins applied by the MOVES, WVU developed SpeedAcceleration tables to estimate the emissions [31, 32]. It was believed that speed related to the road load losses while the combination of speed and acceleration reflected the instantaneous inertial power demand. This method collected emission data for each pre-defined speed and acceleration bin, then averaged the data to obtain the emission value in each bin. The average emission values in each bin were multiplied by the activity data which is the percentage time the vehicle spent in a specific speed-acceleration bin during real-world operation. In this way, the average/integrated emission values for a trip could be obtained by summing the value from the whole range of bins. The emission data for developing the speed-acceleration matrix came from the WVU chassis dynamometer testing. Since the standard chassis dynamometer test cycles did not include grade information, such as uphill or downhill driving, the predicted emission could not completely reflect the real-world operation. Additionally, the existing test cycles were limited, thus a wide range of real-world driving characteristics could not be covered. Non-cycle parameters were not considered in the model, such as altitude, driving terrain and vehicle load/weight, etc. The effect of vehicle weight on emissions could be profound if significant weight variations existed, as well as other non-cycle parameters. It was not convenient to implement these parameters to the model other than introducing more tables or correction factors.

\subsubsection{CMEM}

The Center for Environmental Research and Technology (CE-CERT) at the University of California Riverside (UCR) developed a Comprehensive Modal Emission Model (CMEM) to estimate emissions and fuel consumption. The project started in 1996, sponsored by the National Cooperative Highway Research Program (NCHRP) [33]. The model was originally developed to estimate the second-by-second emissions and fuel consumption for light-duty vehicle (LDV). From 2001, CE-CERT began to expand the model at microscopic, mesoscopic, macroscopic levels for LDV and heavy-duty diesel vehicle (HDD). To develop the HDD instantaneous emission model, data from the Mobile Emission Research Laboratory (MERL) at the CE-CERT 
and HDD truck emission data from Coordinate Research Council Project E-55 were used. Unlike the LDV models which employed the in-house dynamometer test data from 300 real-world vehicles [34], the HDD model used on-road emission data tested by MERL to reflect real-world vehicle operation. The basis for CMEM was the fuel rate derived from the power demand determined by the road-load model. Then emission rates were derived from the fuel rate and were expressed as the product of fuel rate $(\mathrm{g} / \mathrm{s})$, engine-out emission indices ( $\left.\mathrm{g}_{\mathrm{emissions}} / \mathrm{g}_{\mathrm{fuel}}\right)$ and an emission after-treatment pass fraction. The model was composed of six modules, including: 1 ) engine power demand, 2) engine speed, 3) fuel rate, 4) engine control unit, 5) engine-out emissions and 6) after-treatment pass fraction [33, 34]. Since the road-load model determined the power demand, the model had two groups of inputs - input operation variables and model parameters. The input operation variables included second-by-second vehicle speed, grade and accessory load (such as $\mathrm{A} / \mathrm{C}$ ) while the model parameters included specific vehicle parameters (vehicle mass, engine displacement, transmission, etc.), generic vehicle parameters (transmission efficiency, gear ratio, rolling resistance, etc.), and calibrated vehicle parameters (engine friction factor, catalyst pass fraction parameters, etc.) [33, 34]. The outputs of the model included the continuous tailpipe emissions and fuel consumption. Since CMEM employed the physical modal emission modeling approach, it provided insight into the physical and chemical principles that produce emissions and using on-road data reflected actual vehicle operation. However, the model inherently showed complexity and required multiple input parameters to estimate the emissions and fuel consumption, which might not favor of public access. Additionally, the HDD model of CMEM had limited applications, considering the model was applicable only for heavy-duty diesel trucks.

\subsubsection{Neural Network Based Models}

Neural network based models usually required continuous data as inputs such as engine speed, torque, derivatives of engine speed and torque over time, axle speed, coolant temperature, exhaust temperature, oil temperature, intake air temperature, in-cylinder pressure derived variables, air mass, fuel mass, etc.[35-47] and could predict second-by-second emissions or fuel consumption rates.

An artificial neural network (ANN) based emissions model was developed by WVU and it was implemented into the ADvanced VehIcle SimulatOR (ADVISOR) [48] developed by the 
National Renewable Energy Laboratory (NREL) [43]. The model was trained using transient engine dynamometer test data. Inputs included engine speed, torque as well as their first and second derivations over time. The model in combination with ADVISOR was used to predict continuous NOx and $\mathrm{CO}_{2}$ emissions from conventional and hybrid electric vehicles. Results showed good agreement. The model was further validated using additional driving cycles [44].

WVU also applied four different types of ANN to predict continuous emissions from a medium-duty vehicle powered by Fischer-Tropsch synthetic gas-to-liquid compression ignition fuel [45]. These ANN included linear, single hidden layer with sigmoid activation function, nonlinear polynomial, and Gaussian radial basis function. Inputs included vehicle speed, acceleration, rear axle torque, and temperature of emissions. The ANN model based on the radial basis function was found to be better in terms of overall accuracy.

\subsubsection{Summary}

Microscopic models provided more accurate estimations compared with macroscopic ones. However, due to intensive computation, these models usually required much longer time for data processing compared with macroscopic models. On the other hand, macroscopic models were simple and much faster with an acceptable accuracy.

\subsection{Chassis Dynamometer Cycles for Emissions Testing}

The chassis dynamometer test cycles [47-62], were used since emissions and fuel economy are strongly related to driving cycles. Since it was not practical to develop test cycles for all types of vehicles and driving behaviors, it was necessary to develop limited but representative test cycles to mimic driving activities of realistic operation. Specific test cycles have been generated to represent real-world operation in specific applications or localities. For example, the New York Bus cycle (NYBUS) [47] was developed to statistically represent the driving conditions of heavy-duty vehicles in New York City.

Most chassis dynamometer test cycles were recorded as a speed-time trace as shown in Figure 2.2 for the NYBUS cycle. The selected representative test vehicles in this study for backbone modeling (Chapter 6) were operated through twelve chassis dynamometer cycles with multiple repeat runs performed except that one diesel-electric hybrid bus was tested over six cycles. 


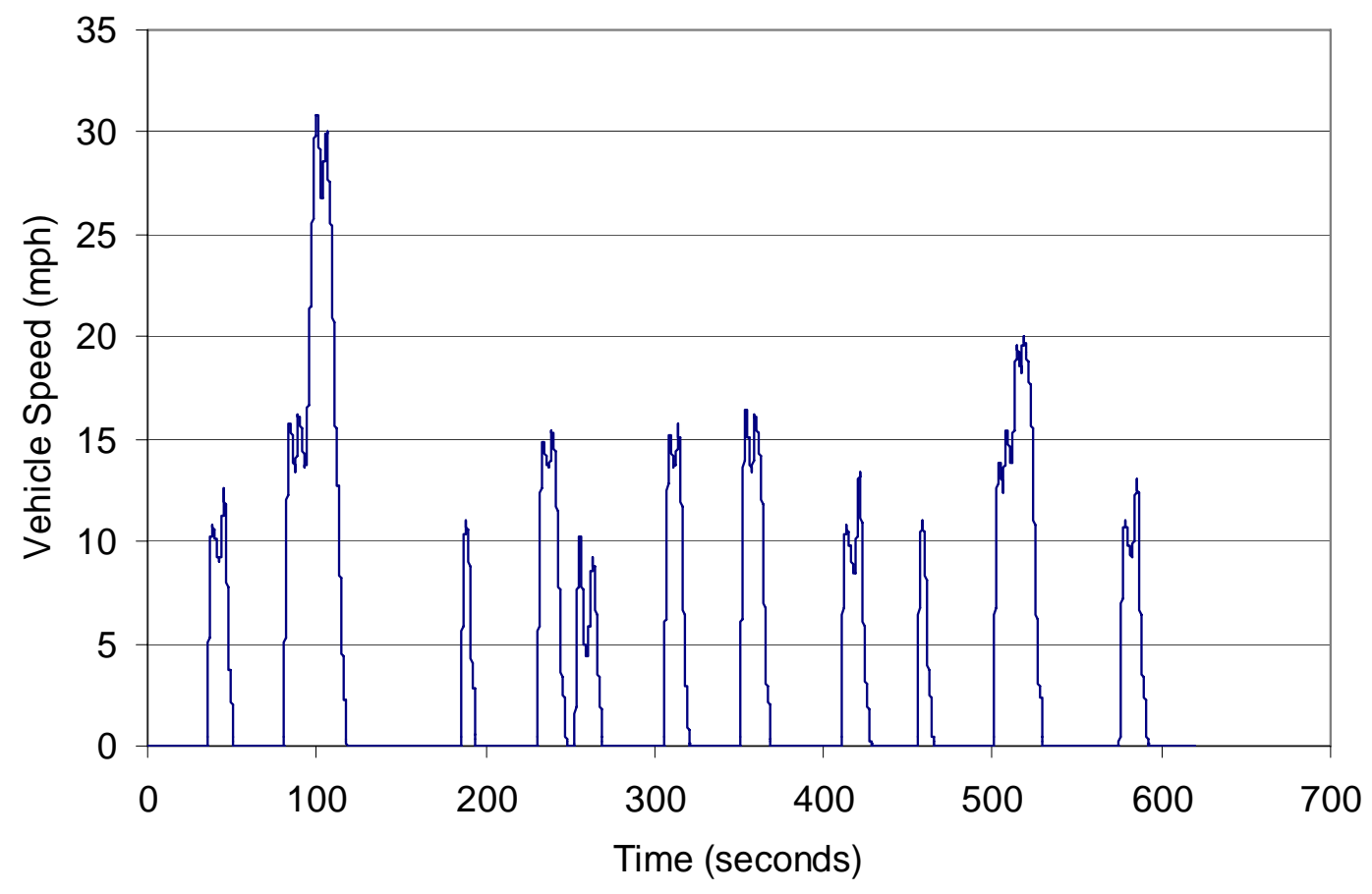

Figure 2.2 New York Bus Cycle

These 12 cycles represented a variety of road conditions in terms of cycle parameters. For example, average speed with idle ranged from the $3.57 \mathrm{mph}$ for the NYBUS cycle to $43.72 \mathrm{mph}$ for the Commuter (COMM) cycle [49]. The percentage idle ranged from 4.32\% for the European Transient Cycle (urban and rural) (ETC_12) [50] to $66.6 \%$ for the NYBUS cycle. It was estimated that the average speed of the whole US transit fleet was $12.72 \mathrm{mph}$ [64]. The Orange County Transit Authority (OCTA) cycle has average speed of 12.08 mph [51, 52] thus it might be used to represent the national fleet-average speed.

\subsubsection{Central Business District Cycle}

The Central Business District (CBD) cycle in Figure 2.3 [49] is composed of 14 repeated idle-acceleration-cruise-deceleration events. The cruise mode is at the speed of $20 \mathrm{mph}$. This kind of driving pattern is too limited to mimic real road operation of transit buses [51]. Although the CBD cycle has its limitations, it is still widely employed for emission tests of transit buses.

For example, WVU database shows that the CBD cycle was used on most emission tests in the earlier MY groups such as MY 1988 - 1997, which contributes to the major reason why the CBD ratio were later selected in this study as an important correction factor for the earlier MY groups. 


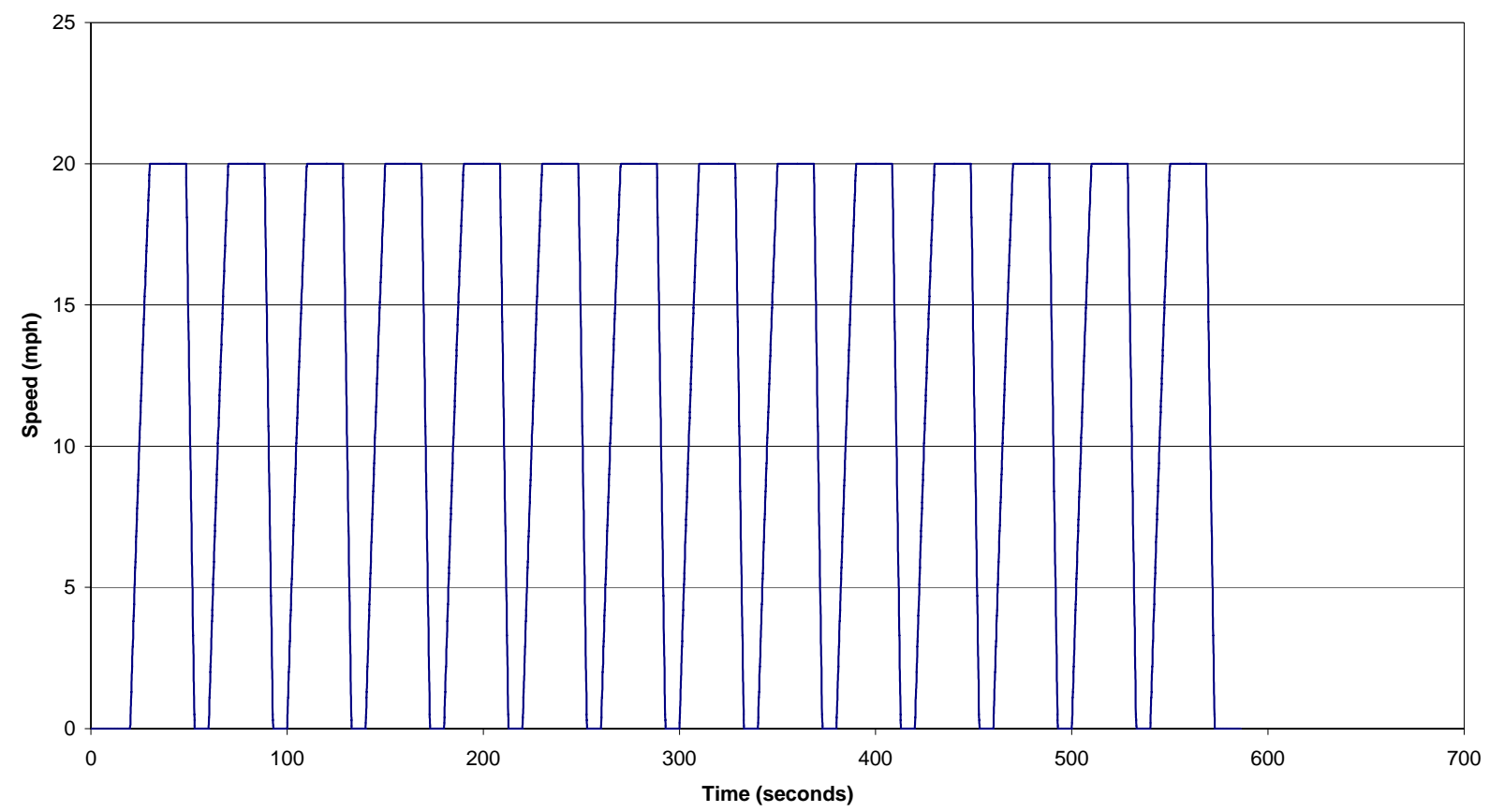

Figure 2.3 Central Business District Cycle

\subsubsection{Braunschweig City Cycle}

The Braunschweig (BRAUN) cycle shown in Figure 2.4 was developed by Technical University of Braunschweig to simulate the transient driving condition with many stops and idles of transit buses driven on the urban routes [53]. Its application to chassis dynamometer test would be diminished because of the introduction of the European Transient Cycle (ETC) cycle.

\subsubsection{The European Transient Cycle}

The European Transient Cycle (ETC) shown in Figure 2.5 was developed by FIGE Institute, Aachen, Germany to mimic the real road characteristics of heavy-duty vehicles on urban, rural and freeway driving [50]. The total duration is 1800 seconds with 600 seconds in each segment. For the first segment, the maximum speed is $30.95 \mathrm{mph}$, while the average speed of rural and freeway driving segments are $44.5 \mathrm{mph}$ and $54.5 \mathrm{mph}$ respectively. In this study, only the first two segments (named as ETC_12) were considered, because the freeway segment could not be executed during the test due to overheating of vehicle tires. As a result, it has the combined average speed of $28.63 \mathrm{mph}$, which represents relatively high-speed operation. 
BRAUN Cycle

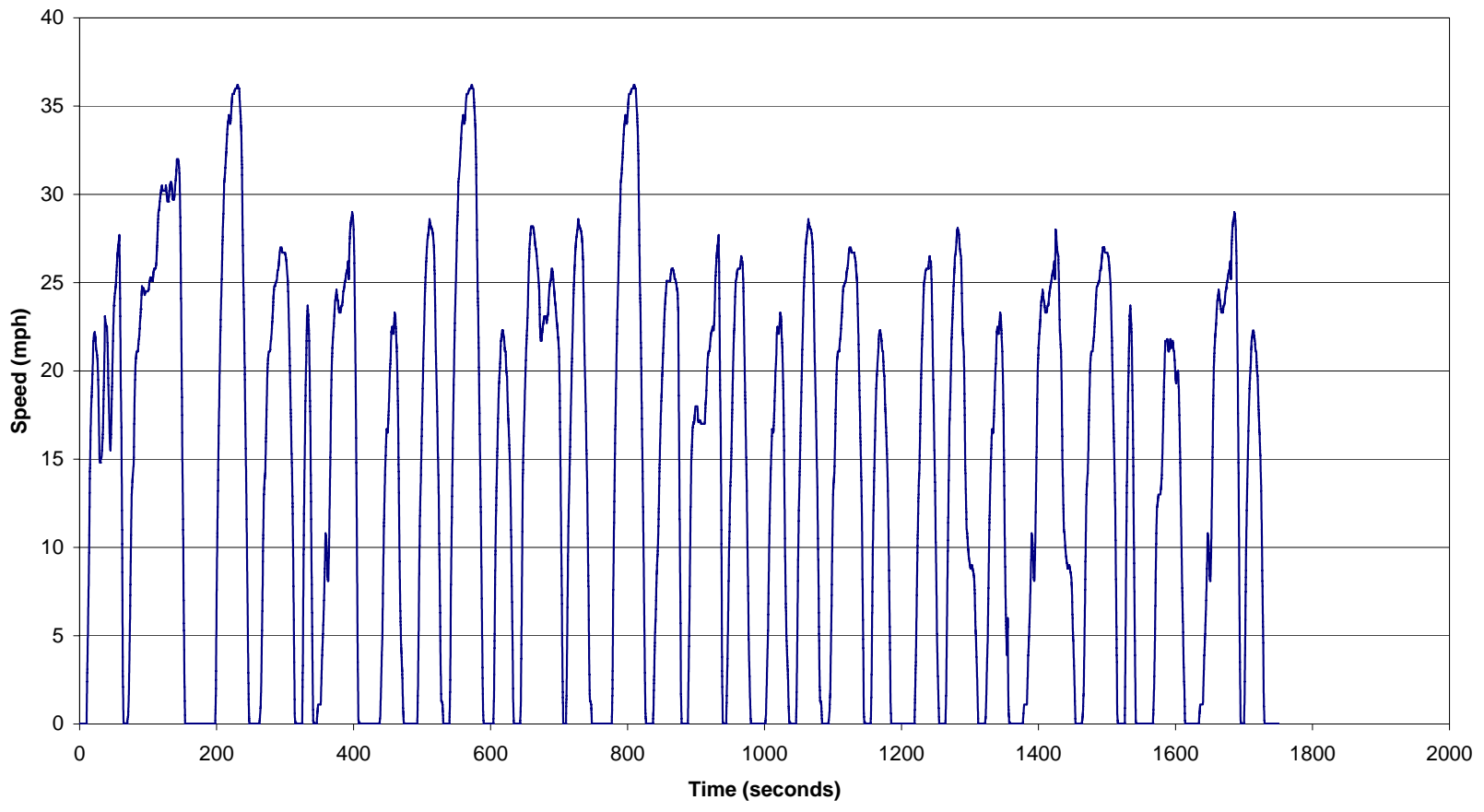

Figure 2.4 Brauschweig City Cycle

ETC Cycle

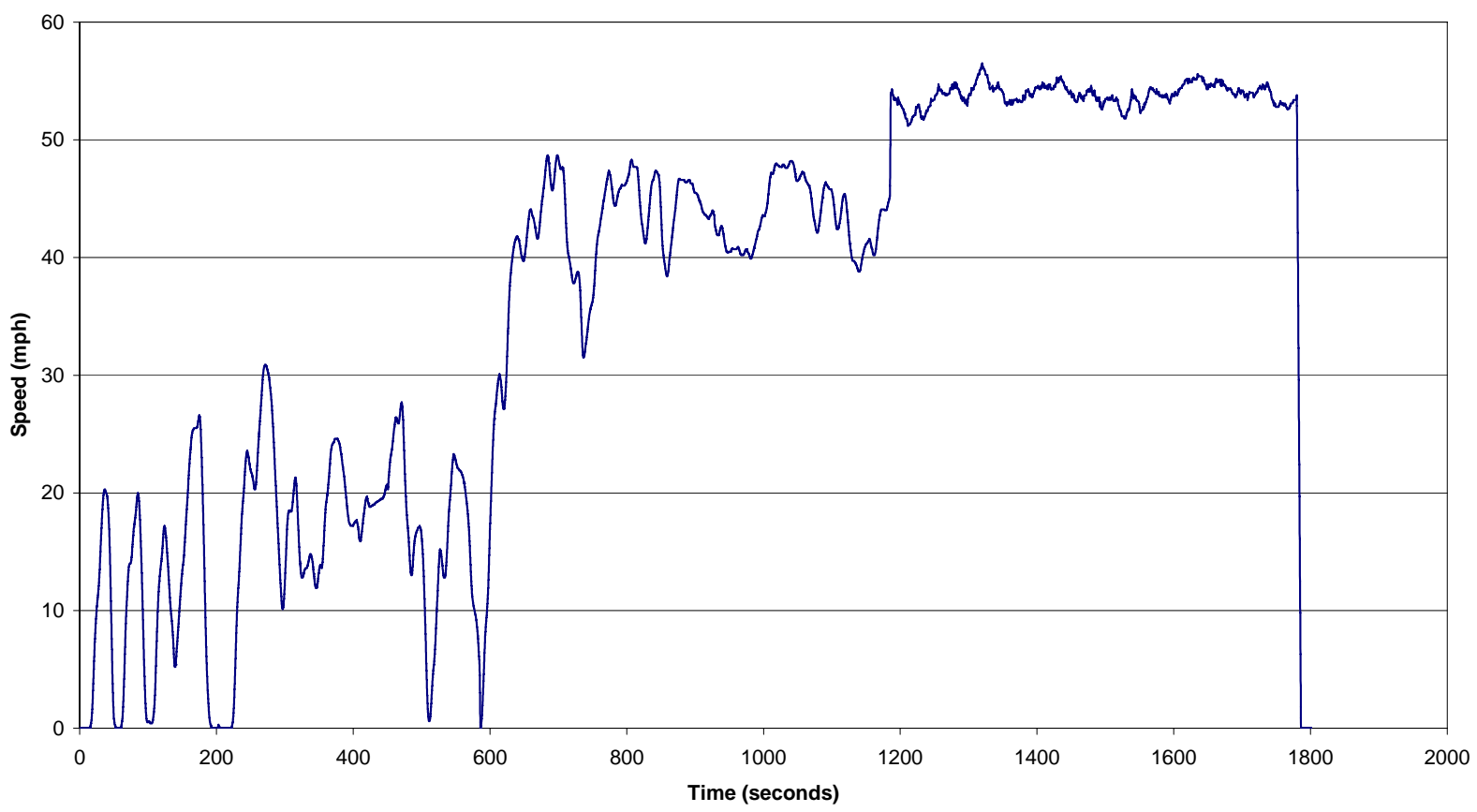

Figure 2.5 European Transient Cycle 


\subsubsection{The New York Bus Cycle}

The New York Bus (NYBUS) cycle presented in Figure 2.2 was developed to mimic the transit bus operation in New York City (NYC). Due to the heavy traffic conditions in NYC, this cycle was generated with slow average speed, frequent stops and fast accelerations. Table 2.4 shows that the cycle has almost 18 stops per mile, which is the highest value of stops per mile among all standard cycles used in this study. Compared with other test cycles, the NYBUS cycle has the slowest average speed of $3.7 \mathrm{mph}$, and because of this characteristic, this cycle might not really represent current transit buses operation in New York City [47].

\subsubsection{The New York Composite Cycle}

Similar to the NYBUS cycle, the New York Composite (NYCOMP) cycle [54] was generated for heavy-duty vehicles representative of real driving patterns in NYC. However, compared to the NYBUS cycle, the NYCOMP cycle has a higher average speed of $8.85 \mathrm{mph}$ and fewer stops. In this way, NYCOMP cycle might be superior to NYBUS cycle for representing present driving conditions in NYC. The cycle speed-time trace is presented in Figure 2.7.

NYBUS Cycle

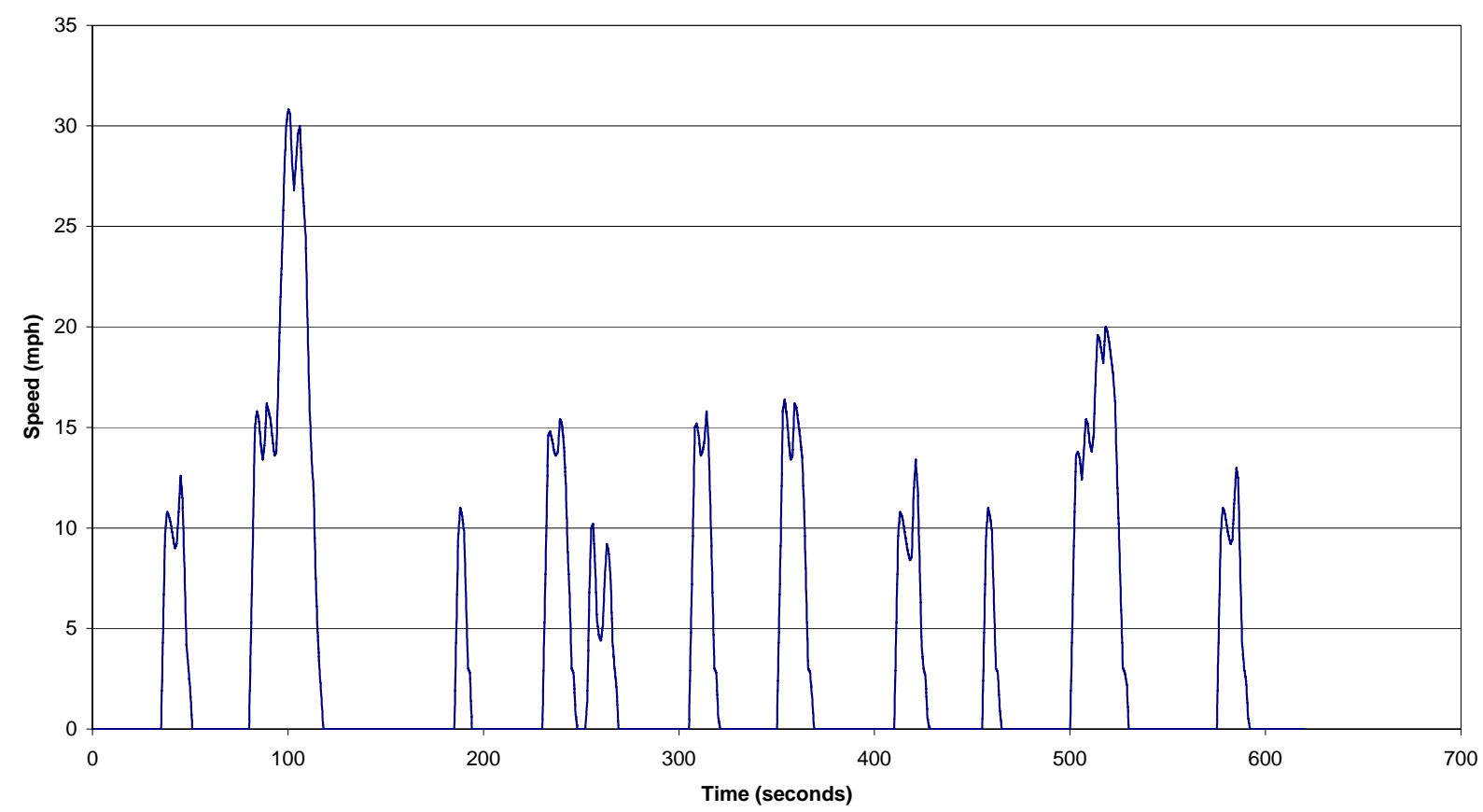

Figure 2.6 New York Bus Cycle 


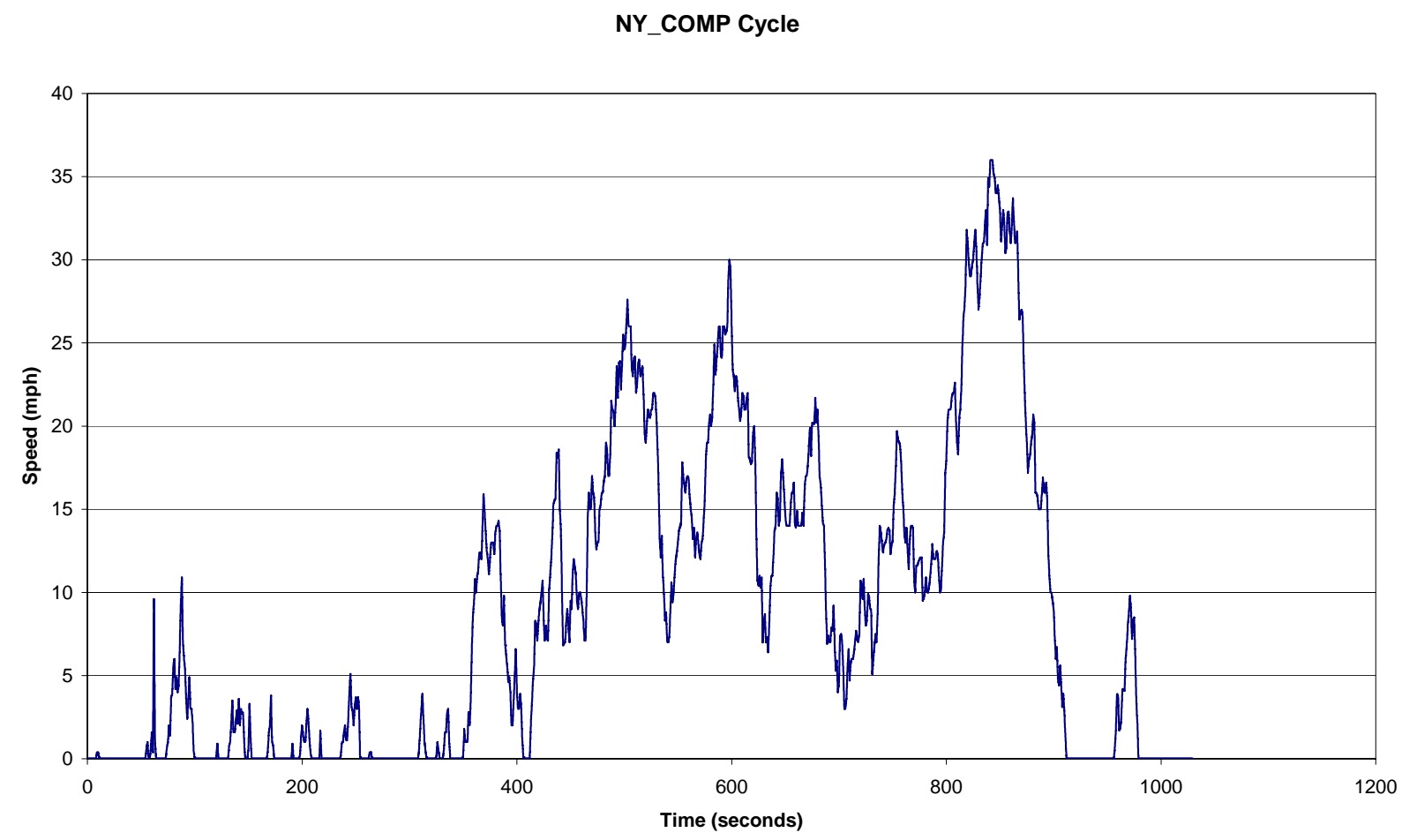

Figure 2.7 New York Composite Cycle

\subsubsection{The Manhattan Cycle}

As one of three recommended test schedules in the SAE J2711 Recommend Practice [51], the Manhattan (MAN) cycle shown in Figure 2.8 was developed by WVU to represent driving conditions of transit buses operating in the NYC Metropolitan area [55]. The cycle data were selected from both conventional and hybrid-electric buses that were operated on different routes in Manhattan. The MAN cycle covers a distance of 2.1 miles and lasts 1083 seconds with an average speed of $6.9 \mathrm{mph}$ consistent with Metropolitan Transportation Authority (MTA) buses operating in NYC [52]. The MAN cycle represents low-speed operation of transit buses [51]. 


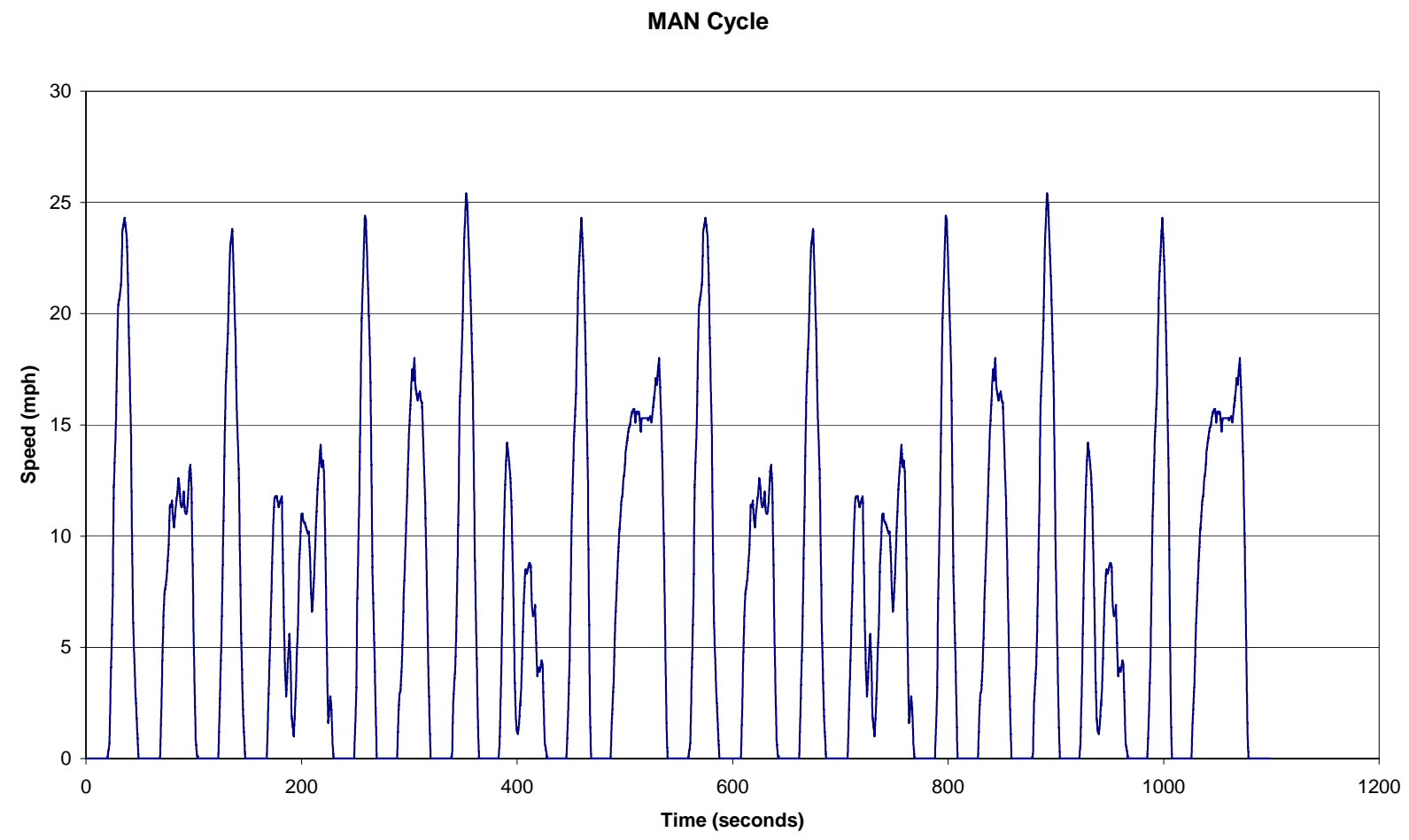

Figure 2.8 Manhattan Cycle

\subsubsection{The Heavy-Duty Urban Dynamometer Driving Schedule}

As another recommended cycle in SAE J2711 [51], the heavy-duty Urban Dynamometer Driving Schedule (UDDS) also referred to as “Test D" was developed by the EPA using Monte Carlo simulation based on binned speed and acceleration data [52]. Test data were collected from transit buses and trucks operating in New York (NY) and Los Angeles (LA) and powered by gasoline and diesel fuel. It includes three sub-cycles: NY non-freeway, LA freeway and LA nonfreeway. It represents the high-speed operation of transit buses [51], showing an average speed of $18.86 \mathrm{mph}$ and a maximum speed of $58 \mathrm{mph}$. The UDDS is shown in Figure 2.9. 


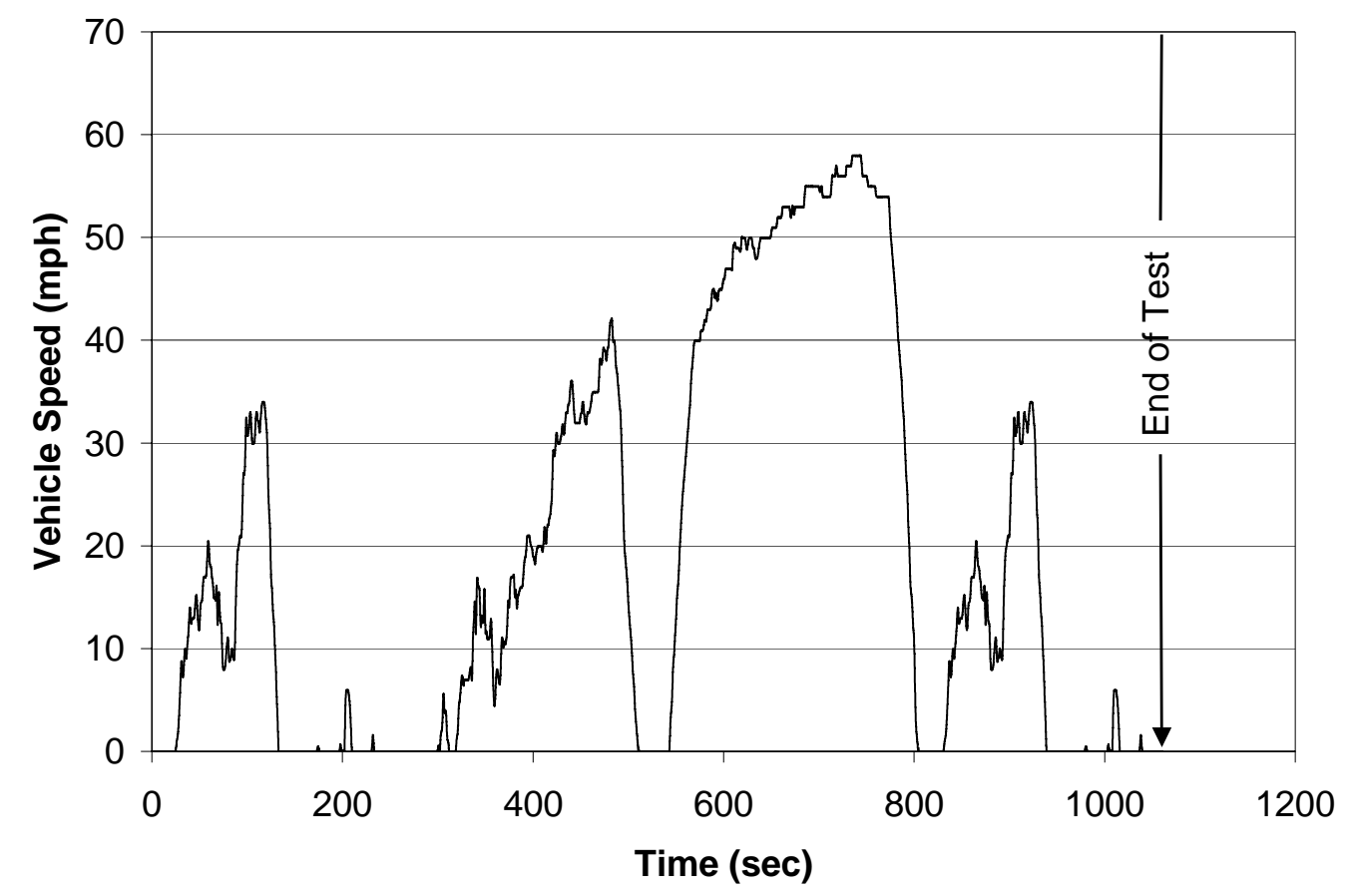

Figure 2.9 The UDDS

\subsubsection{The Washington Metro Area Transit Authority Cycle}

The Washington Metro Area Transit Authority (WMATA) cycle shown in Figure 2.10 [56] was developed to mimic the normal operation of transit buses in the Washington D.C metropolitan area. It has the average speed of $8.32 \mathrm{mph}$, which is slightly lower than the NYCOMP cycle but higher than the MAN cycle. The WMATA cycle lasts 1839 seconds and covers 4.25 miles distance. 


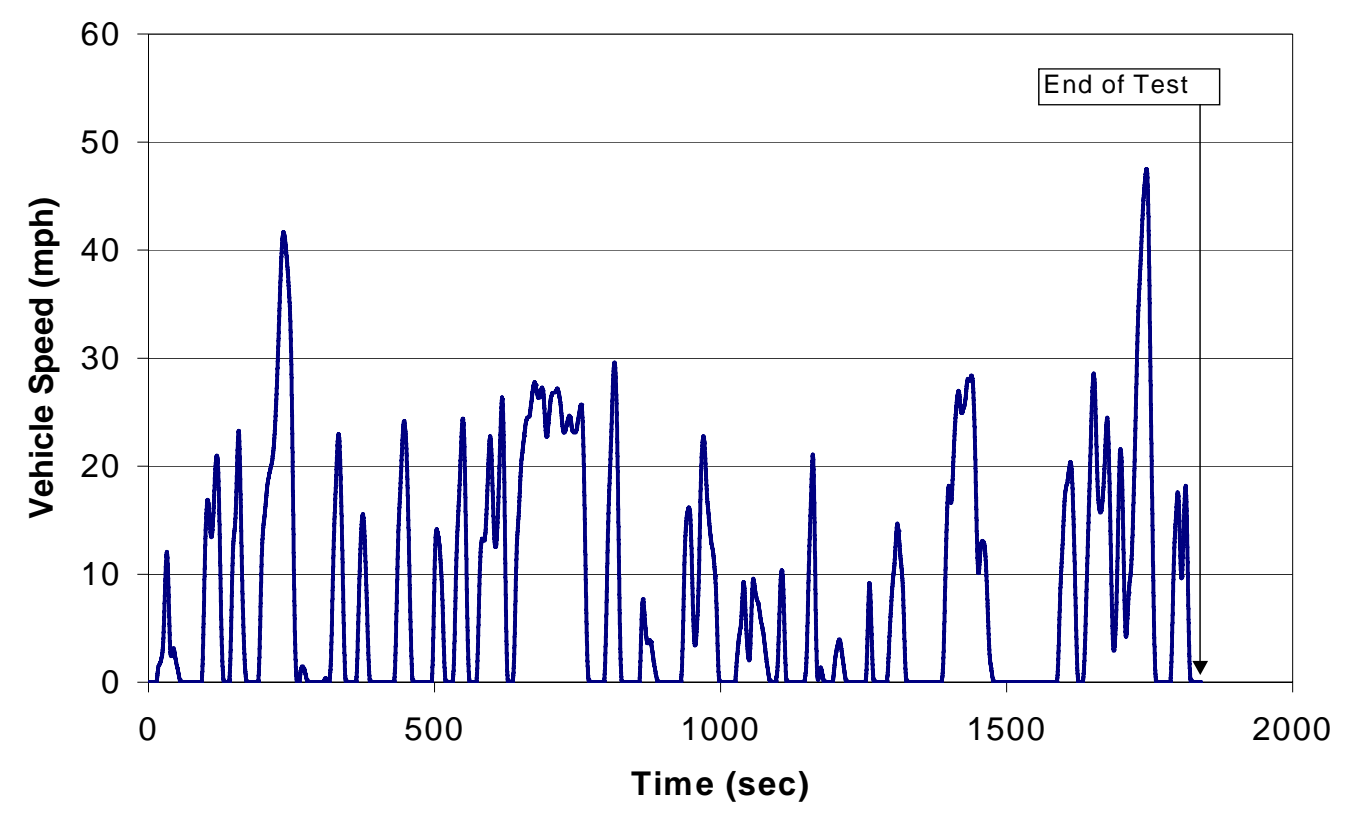

Figure 2.10 WMATA Cycle

\subsection{Cycle Metrics}

Driving characteristics are among the main factors affecting emissions and fuel economy of transit buses. Other important factors included vehicle parameters, fuel types, engine parameters, road conditions, and ambient conditions [65]. To mimic the actual driving conditions of on-road vehicles, chassis dynamometer cycles have been developed [66, 67]. Previous studies, using emissions data from multiple test cycles, showed that distance-specific emissions depended strongly on the characteristics of duty cycles and found that average speed was one of the most important cycle metrics [57-59, 64, 68]. As a consequence, the MOBILE6 [5] and EMFAC [8] models estimated emissions as a function of average speed. Specifically, these macroscopic models calculated emissions based on average speed and vehicle miles traveled. At different average speeds, speed correction factors were used to estimate emissions. Speed correction factors were determined by fitting emissions values to average speed. Previous studies showed the insufficiency of using average speed to evaluate emissions since average speed alone could not comprehensively reflect the cycle characteristics [69, 70]. Other metrics besides average speed, such as percentage idle and average acceleration, have been investigated [70-74]. However, not all important duty cycle metrics were discussed in those studies. Correlation and regression analyses were provided in Chapter 4 to investigate the effects of cycle characteristics on distance specific emissions of $\mathrm{CO}, \mathrm{CO}_{2}$, $\mathrm{NOx}, \mathrm{HC}$, PM, and fuel economy. 
Twelve target standard cycles were frequently used in this study and their characteristics were summarized in Table 2.4. In total, Table 2.4 lists 13 cycle metrics (parameters). Beside those metrics calculated based on target cycle speed-time traces, aerodynamic speed, characteristic acceleration and kinetic intensity were derived based on the road load equation [63].

Table 2.4 Target Cycle Parameters

\begin{tabular}{|c|c|c|c|c|c|c|c|c|}
\hline Cycle & $\begin{array}{l}\text { Duration } \\
\text { (seconds) }\end{array}$ & $\begin{array}{c}\text { Distance } \\
\text { Traveled } \\
\text { (miles) } \\
\end{array}$ & $\begin{array}{l}\text { Average } \\
\text { Speed } \\
\text { with Idle } \\
\text { (mph) }\end{array}$ & $\begin{array}{l}\text { Average } \\
\text { Speed } \\
\text { without } \\
\text { Idle (mph) }\end{array}$ & $\begin{array}{c}\text { Percentage } \\
\text { Idle }\end{array}$ & $\begin{array}{c}\text { Number } \\
\text { of Stops } \\
\text { per Mile }\end{array}$ & $\begin{array}{c}\text { Standard } \\
\text { Deviation of } \\
\text { Speed with } \\
\text { Idle (mph) }\end{array}$ & $\begin{array}{c}\text { Standard } \\
\text { Deviation of } \\
\text { Speed } \\
\text { without Idle } \\
\text { (mph) }\end{array}$ \\
\hline ART & 291.6 & 2.00 & 24.71 & 29.55 & $16.39 \%$ & 2.00 & 15.64 & 12.19 \\
\hline BEELINE & 1724 & 6.79 & 14.17 & 19.29 & $26.54 \%$ & 3.54 & 14.74 & 14.04 \\
\hline BRAUN & 1750 & 6.73 & 13.85 & 18.48 & $25.04 \%$ & 4.31 & 11.35 & 9.30 \\
\hline CBD & 586 & 2.01 & 12.36 & 15.71 & $21.35 \%$ & 6.96 & 8.46 & 6.19 \\
\hline COMM & 329.6 & 4.00 & 43.72 & 49.71 & $12.04 \%$ & 0.25 & 19.46 & 11.46 \\
\hline CSHVR & 1700.1 & 6.68 & 14.15 & 18.33 & $22.80 \%$ & 1.95 & 13.05 & 12.01 \\
\hline ETC_12 & 1200 & 9.54 & 28.63 & 29.93 & $4.32 \%$ & 0.42 & 15.84 & 14.95 \\
\hline KCM & 1964 & 12.75 & 23.38 & 28.42 & $17.75 \%$ & 1.88 & 18.09 & 15.95 \\
\hline MAN & 1098.7 & 2.07 & 6.77 & 10.66 & $36.52 \%$ & 9.68 & 7.33 & 6.56 \\
\hline NYBUS & 620 & 0.61 & 3.57 & 10.69 & $66.60 \%$ & 17.89 & 6.41 & 6.86 \\
\hline NY-COMP & 1029 & 2.51 & 8.77 & 12.85 & $31.76 \%$ & 7.58 & 9.44 & 8.84 \\
\hline OCTA & 1950 & 6.54 & 12.08 & 15.52 & $22.17 \%$ & 4.74 & 10.33 & 9.14 \\
\hline PARIS & 1910 & 3.50 & 6.59 & 9.91 & $33.46 \%$ & 13.44 & 7.28 & 6.83 \\
\hline TRANS3 & 668 & 2.85 & 15.36 & 18.21 & $15.64 \%$ & 1.75 & 13.38 & 12.66 \\
\hline UDDS & 1060 & 5.54 & 18.83 & 28.04 & $32.84 \%$ & 2.89 & 19.82 & 18.07 \\
\hline WMATA & 1839 & 4.25 & 8.32 & 13.47 & $38.27 \%$ & 6.12 & 10.31 & 10.14 \\
\hline
\end{tabular}

Table 2.4 Target Cycle Parameters - Cont’d

\begin{tabular}{|c|c|c|c|c|c|c|c|}
\hline Cycle & $\begin{array}{c}\text { Average } \\
\text { Acceleration } \\
\left.\text { (ft/sec }{ }^{2}\right) \\
\end{array}$ & $\begin{array}{c}\text { Maximum } \\
\text { Acceleration } \\
\left(\mathrm{ft} / \mathrm{sec}^{2}\right)\end{array}$ & $\begin{array}{c}\text { Average } \\
\text { Deceleration } \\
\left(\mathrm{ft} / \mathrm{sec}^{2}\right)\end{array}$ & $\begin{array}{c}\text { Maximum } \\
\text { Deceleration } \\
\left(\mathrm{ft} / \mathrm{sec}^{2}\right)\end{array}$ & $\begin{array}{c}\text { Aerodynamic } \\
\text { Speed (mph) } \\
\end{array}$ & $\begin{array}{c}\text { Characteristic } \\
\text { Acceleration } \\
\left(\mathrm{ft} / \mathrm{sec}^{2}\right)\end{array}$ & $\begin{array}{c}\text { Kinetic } \\
\text { Intensity } \\
\left(\mathrm{mile}^{-1}\right) \\
\end{array}$ \\
\hline ART & 2.02 & 3.67 & 6.45 & 7.33 & 35.58 & 0.65 & 1.26 \\
\hline BEELINE & 2.06 & 7.33 & 2.58 & 10.27 & 32.03 & 0.88 & 2.10 \\
\hline BRAUN & 2.08 & 8.07 & 2.80 & 11.73 & 24.17 & 0.72 & 3.02 \\
\hline CBD & 2.87 & 3.67 & 6.38 & 7.33 & 18.55 & 0.57 & 4.04 \\
\hline COMM & 1.37 & 3.67 & 6.67 & 18.33 & 52.84 & 0.15 & 0.14 \\
\hline CSHVR & 1.49 & 4.40 & 1.83 & 5.87 & 27.72 & 0.56 & 1.79 \\
\hline ETC_12 & 1.14 & 13.20 & 1.26 & 8.07 & 39.16 & 0.31 & 0.50 \\
\hline KCM & 1.81 & 14.67 & 2.86 & 12.47 & 40.16 & 0.54 & 0.83 \\
\hline MAN & 2.04 & 7.33 & 2.59 & 8.80 & 15.78 & 0.94 & 9.24 \\
\hline NYBUS & 4.09 & 9.53 & 2.39 & 7.33 & 16.64 & 1.25 & 11.07 \\
\hline NY-COMP & 1.72 & 13.93 & 1.94 & 13.20 & 20.69 & 0.77 & 4.42 \\
\hline OCTA & 1.88 & 5.87 & 2.61 & 8.07 & 22.10 & 0.72 & 3.60 \\
\hline PARIS & 1.90 & 7.33 & 2.08 & 13.93 & 16.05 & 0.83 & 7.93 \\
\hline TRANS3 & 1.34 & 4.40 & 1.78 & 4.40 & 29.57 & 0.50 & 1.40 \\
\hline UDDS & 1.78 & 8.80 & 1.99 & 8.07 & 42.49 & 0.50 & 0.68 \\
\hline WMATA & 1.74 & 4.40 & 2.10 & 6.60 & 23.22 & 0.77 & 3.51 \\
\hline
\end{tabular}

Unlike the conventional cycle metrics that were directly derived from the speed-time

trace, aerodynamic speed, characteristic acceleration, and kinetic intensity were derived from the 
road load equation and were intended to be indicative of power and fuel consumed. Due to detailed derivations and background information provided in previous studies [63, 75], this section presents a brief introduction of these three metrics.

Equation 2.2 - Equation 2.4 were derived in $[63,75]$ to represent characteristic acceleration, aerodynamic speed, and kinetic intensity, respectively.

$$
\begin{aligned}
& a_{\text {char }}=\frac{\sum_{j=1}^{N-1} \text { positive }\left(\frac{1}{2} \times\left(v_{j+1}^{2}-v_{j}^{2}\right)+g \times \Delta h_{j, j+1}\right)}{D} \\
& v_{\text {aero }} \cong \sqrt{\frac{\sum_{j=1}^{N-1} \overline{v_{j, j+1}^{3}} \times \Delta t_{j, j+1}}{D}} \\
& k i=\frac{\sum_{j=1}^{N-1} \text { positive }\left(\frac{1}{2} \times\left(v_{j+1}^{2}-v_{j}^{2}\right)+g \times \Delta h_{j, j+1}\right)}{\sum_{j=1}^{N-1} \overline{v_{j, j+1}^{3}} \times \Delta t_{j, j+1}} \\
& \Delta t_{j, j+1}=t_{j+1}-t_{j} \\
& \Delta h_{j, j+1}=h_{j+1}-h_{j} \\
& \overline{v_{j, j+1}^{3}}=\frac{v_{j+1}^{3}+v_{j+1}^{2} \times v_{j}+v_{j}^{2} \times v_{j+1}+v_{j}^{3}}{4}
\end{aligned}
$$

Equation 2.5

Equation 2.7

where $v_{\mathrm{j}}$ is the instantaneous vehicle speed at time step $j, t$ is time, $g$ is the gravitational acceleration, $h_{\mathrm{j}}$ is vehicle elevation above a fixed reference at time step $j$, and $D$ is the driving distance of the cycle. Gradient effects were neglected in this study, thus the height difference term in Equation 2.2 and Equation 2.4 were omitted.

Equation 2.2 shows that only positive accelerations were considered. After neglecting road grade effects, the characteristic acceleration indicated the specific kinetic energy per unit mass and distance to accelerate a vehicle over a duty cycle. The characteristic acceleration was equal to the actual vehicle acceleration if the vehicle increased its speed at a constant rate. The square of aerodynamic speed directly reflected the effects of aerodynamics on fuel economy and it was equal to the actual vehicle speed if the vehicle was driven at a constant speed. Kinetic intensity was related to fuel savings of hybrid vehicles over their conventional counterpart vehicles tested on the same cycles and thus it gave an indication of whether hybridization would 
result in fuel savings or not for a particular duty cycle. Equation 2.4 shows that the kinetic intensity is the ratio of characteristic acceleration to the square of aerodynamic speed. A cycle with larger characteristic acceleration and smaller aerodynamic speed resulted in higher kinetic intensity and was better for hybridization [63].

These three cycle metrics were originally intended to connect with fuel consumption and be used to differentiate duty cycles on a fuel usage basis for hybrid vehicle applications [63]. It was evident that all three metrics were independent of vehicle information; thus, they were the inherent characteristics of duty cycles. Since they were derived from the road load equation and connected to energy usage, these cycle metrics were believed to have some relationships with emissions and fuel economy. These relationships were discussed in Chapter 4.

\subsection{Cycle Development}

To develop dynamometer test cycles for evaluation of emissions from heavy-duty vehicles, various approaches have been used by previous researchers. One of two major methods uses Monte Carlo simulation [61]. A typical example is the creation of the UDDS using Monte Carlo simulation based on a statistically binned speed and acceleration matrix. Test data were logged from transit buses and trucks that were operated in New York (NY) and Los Angels (LA) and fueled by gasoline and diesel. A complete cycle was developed to reflect the real-world operations and it was composed of three sub-cycles: NY non-freeway, LA freeway and LA nonfreeway [52].

More recently, a microtrip based method has been widely used to create a candidate cycle with the lowest Root Mean Square (RMS) value to best statistically represent the vehicle activity database [56]. A microtrip can be defined as a short trip [67] or a shorter segment with speed greater than $0.5 \mathrm{mph}$. Microtrips were randomly selected from the activity database then reordered and combined together to form a new candidate cycle. The activity database was usually composed of continuous speed-time traces from multiple heavy-duty vehicles operating in the specific area. A variety of statistical measures were used as criteria to compare the candidate cycle with the activity database. Those statistical parameters included but were not limited to average speed with or without idle, the standard deviation of vehicle speed with or without idle, kinetic energy, and percentage idle, etc. [56, 62, 66, 67]. Idle periods can be added later or can be included as a portion of a microtrip. The cycle creation process can be applied to the whole 
vehicle activity database to generate a whole new cycle. It can also be applied to the individual mode based activity database to separately generate a new cycle for each mode behavior then combine all of best cycles from each mode to form a new cycle [66]. Different modes were defined based on vehicle speed, including an idle mode, creep mode for very low speed, transient mode for moderate speed and cruise mode for high speed. The lowest RMS method was usually used to select the best candidate as the new cycle. Previous studies have been related to creating new dynamometer test cycles. However, none of previous work has been found to attempt to generate cycles with a complete emissions profile. 


\section{IBIS DEVELOPMENT STRATEGIES}

Sponsored by the FTA, IBIS was developed by WVU to evaluate emissions inventories and fuel economy for transit buses, providing current transit agencies useful information for new bus procurement. IBIS included three components: a searchable database of emissions test results of transit buses, a transit fleet emissions model and a life cycle cost model. This study was focused to develop a novel and simple macroscopic model called IBIS transit fleet emissions model (abbreviately IBIS emissions model or IBIS model) [76]. The development of IBIS mainly relied on the searchable transit bus emissions database, a database with chassis dynamometer testing data provided by the Center for Alternative Fuels, Engines, and Emissions (CAFEE) at WVU. The test facilities with the database involved in this study is introduced later in this chapter. The preliminary version of IBIS could be accessed online [77] while it will be updated when new models associated with new data sources become available. Figure 3.1 shows a screenshot of the login page of the IBIS online version and Figure 3.2 provides a screenshot of the online interface of IBIS transit fleet emissions model. To be noted, since the IBIS emissions model has not been fully uploaded online, the online version uses only standard cycles as inputs for driving characteristics and the option of customized cycle inputs will be implemented online in the future. 


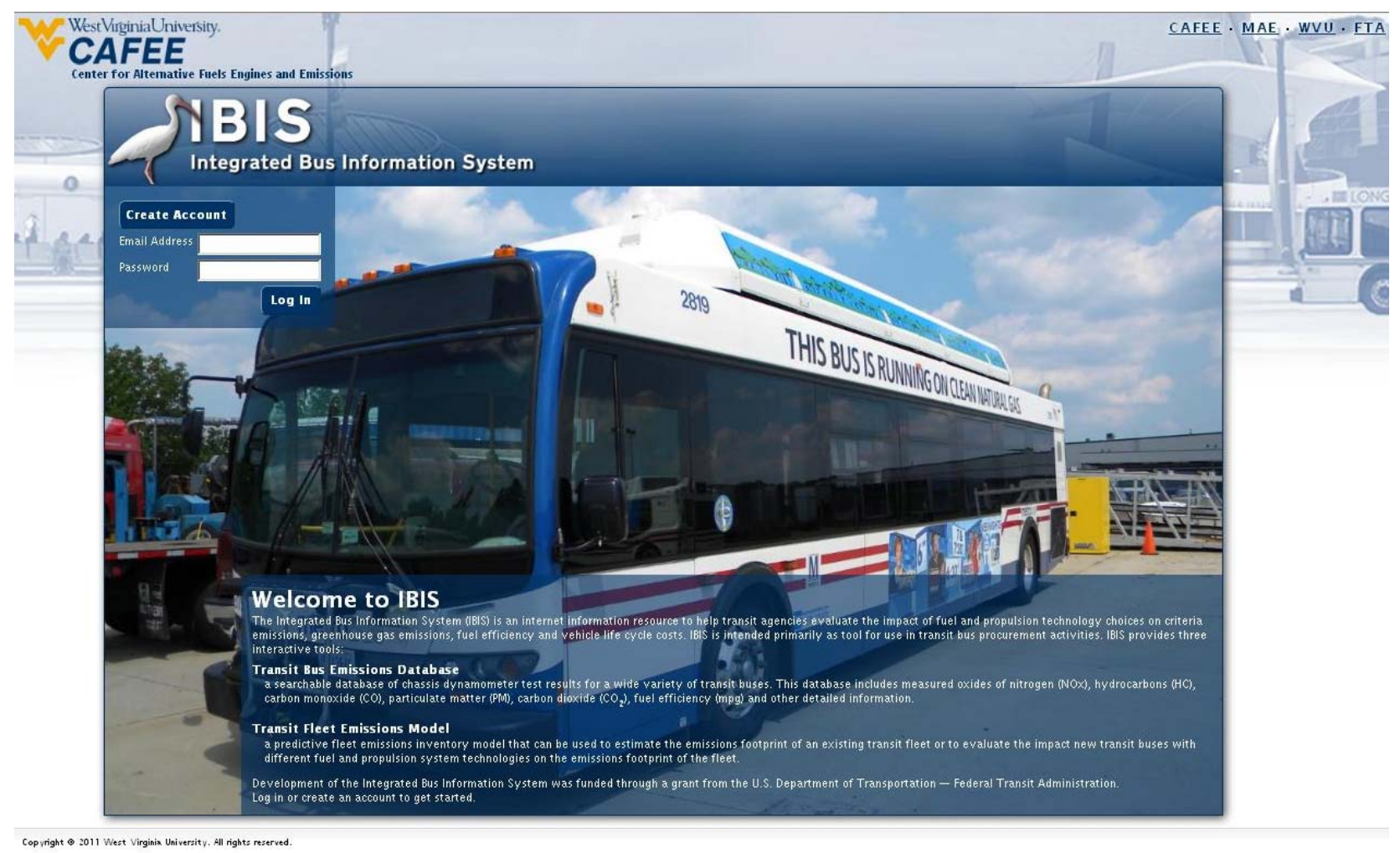

Figure 3.1 Screenshot of Preliminary Online Version of IBIS Login Page

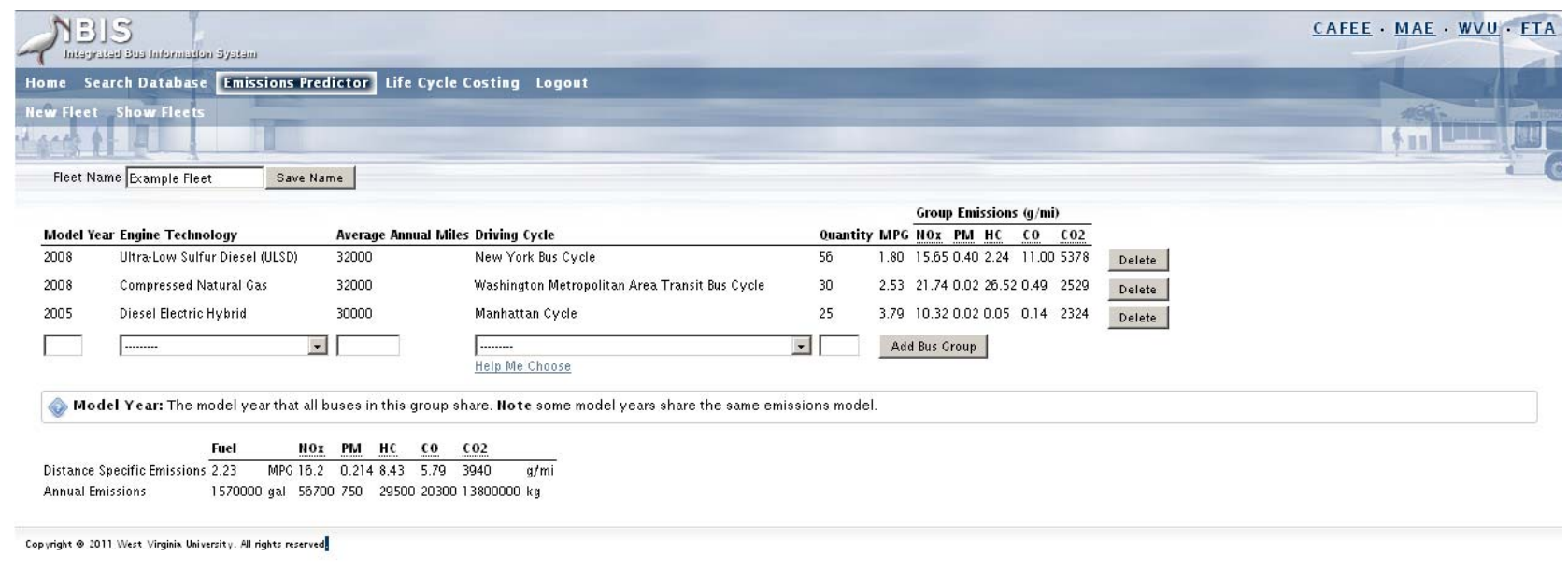

Figure 3.2 Screenshot of Preliminary Online Version of IBIS Emissions Model Interface

\subsection{IBIS Model Inputs}

Many factors affect emissions and fuel economy of transit buses. IBIS model inputs could be organized into three categories which were vehicle parameters, driving characteristics, and external operational conditions. Vehicle parameters are listed as follows:

- Type of Fuel (or Propulsion)

- Vehicle Length 
- Vehicle Model Year (VMY) Group

- Curb Weight

- Seating Capacity (N/A)

- Rated Power (N/A)

- Number of Cylinders (N/A)

- Engine Displacement (N/A)

- Transmission Type

- Type of Heating System (N/A)

- After-treatment Technologies (Diesel Buses)

Parameters denoted as N/A values had not been considered or were set as default values in the IBIS model which would be future research topics once relevant testing data became available. Parameters had their specific value options which were introduced as drop down menus in the friendly user interface. For example, in this study, fuel (propulsion) technology types included diesel, compressed natural gas (CNG), and hybrid diesel-electric powered transit buses due to their majority in the transit field. For diesel buses, three types of diesel fuel including diesel \#1 (D1), diesel \#2 (D2), and ultra low sulfur diesel \#1 (ULSD1) were grouped as the same fuel type because they generated comparable emissions and fuel economy values. Other types of fuel powered buses might be found in-use for transit agencies, but due to their minority only three types of major fuel were included in the study at this point.

Most of the heavy-duty transit buses are 40 and 60 feet in length, which were the focus of this study. The VMY was actually organized in several vehicle model year (MY) groups which were based on the EPA's regulations on emissions. Those buses in each MY group were assumed to be similar buses in terms of emissions and fuel consumption if no significant difference existed in other factors. Currently, seven MY groups were considered as follows:

- MY Group I: 1988-1991

- MY Group II: 1992-1993

- MY Group III: 1994-1997

- MY Group IV: 1998-2002

- MY Group V: 2003-2006

- MY Group VI: 2007-2009 
For newer MY groups, backbone models were developed for different fuel types of vehicles in individual MY groups. For older MY groups, correction factors were determined and applied to the backbone models and emissions and fuel economy values were adjusted for each MY group.

From previous studies [73], curb weight and seating capacity were found to affect emissions and fuel economy. If a 40 -foot bus with the full capacity of 60 passengers is considered and the curb weight is about 29,000 lbs, the full load will be about 38,000 lbs if each passenger weighs $150 \mathrm{lbs}$ on average. The half load is about 33,500 lbs with 30 passengers. In Chapter 7, impact of the vehicle weight on emissions and fuel economy is discussed.

For older diesel transit buses, after-treatment technologies might be introduced as retrofitted devices to reduce emissions. In this study, as one of major after-treatment technologies diesel particulate filter (DPF) was included in the IBIS emissions model.

The main parameters in driving characteristics also called duty cycle characteristics included average speed with idle (or average speed), number of stops per mile, percentage idle, standard deviation of vehicle speed with idle, and kinetic intensity. Chapter 4 provides correlation analysis of their impact on emissions and fuel economy and discusses how they were selected as inputs of the IBIS model.

The external operational conditions mainly referred to road grade, ambient temperature, altitudes, geographical location, and seasonal conditions. The parameters of this category have not been considered in this study due to the limited data availability for the research.

\subsection{IBIS Model Outputs}

With the limited information required as the IBIS inputs, the model was intended to estimate or predict emissions and fuel economy for a single bus scenario or a fleet if the fleet profile could be provided. The model outputs included emissions of $\mathrm{CO}_{2}, \mathrm{HC}, \mathrm{CO}, \mathrm{NOx}$, and PM in gram/mile as the basic metric while fuel economy was expressed in mpg. Also, total mass emitted and fuel consumed per year from the fleet could be provided.

\subsection{General Architecture of IBIS Model Development}

The chassis dynamometer data were relatively limited compared with the on-road/onboard emission test data due to the relatively high cost and effort involved in the tests. Most 
importantly, unlike the on-road tests, standard cycles developed for chassis dynamometer tests were limited and might not completely reflect operating conditions of transit buses. Considering the limitation of the data source, a cycle generation approach using micro-trips was implemented in the development of the IBIS model to expand the database, the details of which are discussed in Chapter 5.

Based on the expanded database, IBIS used the driving characteristics to determine the values of emissions and fuel economy for a particular bus in a specified MY group and fuel type. The models at this stage were called backbone models which primarily employed polynomial regressions. The basic principle of the backbone models was to estimate the emissions and fuel economy using selected duty cycle parameter pairs which were average speed in combination with one of other selected parameters from the driving characteristics. For instance, if average speed, percentage idle and standard deviation of vehicle speed were considered as inputs, then one pair consisting of average speed and percentage idle and the other pair consisting of average speed and standard deviation of vehicle speed would be fitted separately using polynomial regression. Then the estimates from each pair of cycle parameters were averaged and provided as results from backbone models. More details regarding IBIS backbone modeling are provided in Chapter 6.

Input parameters from other categories were considered as correction factors applied to the backbone model to determine the final values of outputs. For instance, vehicle parameters such as weight, after-treatment technologies (diesel buses) and model year group were analyzed and correction factors for each parameter were determined with assumptions that they had independent effects on the outputs. These are discussed in Chapter 7. If fleet information could be provided such as the number of each type of the buses and VMT, then the total emissions and fuel consumption could be calculated. To assist in the IBIS model development, computational tools were developed by Marlowe [78].

The general form of IBIS emissions models can be expressed as:

$$
Y=f_{\text {backbone }}\left(x_{1}, \cdots x_{i}\right) \times c_{1} \times c_{2} \cdots c_{i}
$$

Equation 3.1

where $f_{\text {backbone }}$ were the backbone models, $x_{i}$ were cycle metrics and $c_{i}$ were the corrections factors. The general structure of the IBIS emissions model development is shown in Figure 3.3. 
Figure 3.3 Architecture of IBIS Emissions Model

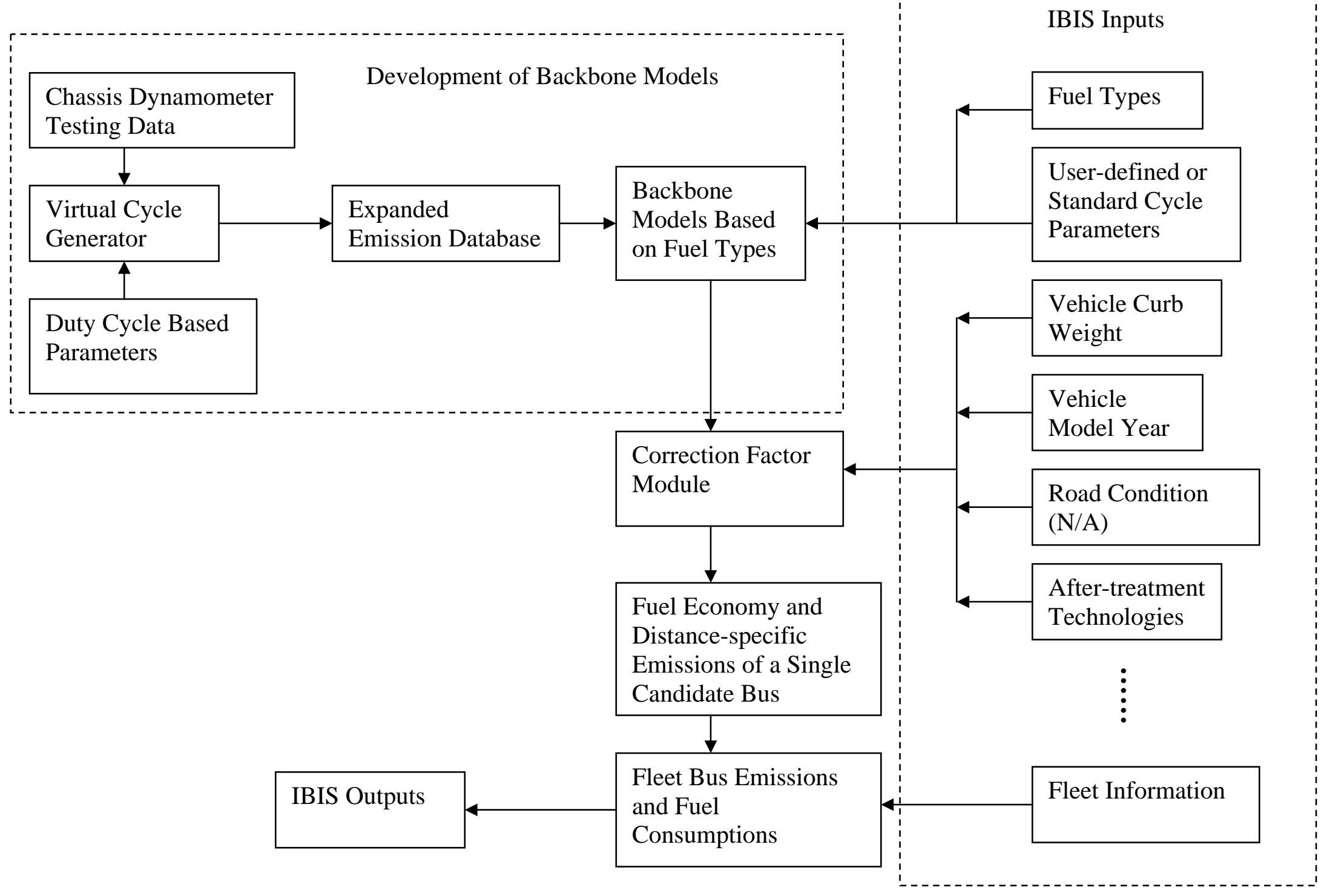




\subsection{Testing Facilities and Data Collection}

\subsubsection{Description of WVU Testing Facilities}

The data used in this study were mainly drawn from emission tests performed using the WVU Transportable Heavy-Duty Vehicle Emission Laboratory (TransLab). The laboratory was designed to perform on-site measurement of emissions and it consists of two trailers, one of which was equipped with a dynamometer including a set of rollers, flywheels, and power absorbers, while the other housed emission measurement facilities including the emissions analyzers, data acquisition system, and sampling system. The original laboratory was designed to meet the criteria outlined in the CFR 40, Part 86, Subpart N [60] where applicable to chassis dynamometer testing. The current emissions testing system followed CFR 40, Part 1065 [79]. The test vehicle was driven by a certified driver on the chassis dynamometer.

The emissions from the vehicle tailpipe were diluted and mixed with ambient air in the dilution tunnel. The specific analyzers were employed to measure the different emissions. Both $\mathrm{CO}$ and $\mathrm{CO}_{2}$ were detected by non-dispersive infrared (NDIR) gas analyzers. These gases would absorb infrared radiation within a specific wavelength range unique to the gas. An NDIR detected the amount of infrared radiation absorbed to determine the amounts of these gases in the sample stream. HC was measured using a heated flame ionization detector (HFID). In an HFID the sample was burned in a hydrogen/helium flame. As it was burned ions were released and collected on electrodes producing a flow of electrical current in the instrument. The current flow was proportional to the amount of hydrocarbons in the sample. The wet chemiluminescent analyzer was employed to characterize NOx emissions, which was based on the principle that a certain chemical reaction could emit light. In a chemiluminescent analyzer, nitrous oxide was converted to nitrogen dioxide through a gas-phase reaction with ozone. About $10 \%$ of the nitrogen dioxide immediately reverted to nitrous oxide releasing a photon in the process. An optical detector measured the photons, which were proportional to the amount of nitrous oxide in the sample. To measure oxides of nitrogen, all the nitrogen dioxide in the sample was initially converted to nitrous oxide prior to entering the analyzer. For PM data, the total weight was measured gravimetrically after each test run. Continuous fuel consumption rates were calculated based on carbon balance from the exhaust emissions. More details on the laboratory design and operation were presented in references [65, 71-73, 80-82]. 


\subsubsection{Description of WVU Database}

WVU CAFEE has performed chassis dynamometer emission tests nationwide for heavyduty vehicles, including transit buses, school buses, refuse trucks, dump trucks, and tractors [83]. In total, over ten thousand test runs have been performed and involved over 1,000 heavy-duty vehicles including buses and trucks with VMY from the early 1980's. About 30 different fuel types and 140 test cycles have been tested on those vehicles. CAFEE has built the largest national database of emissions and fuel economy data from heavy-duty vehicles [83].

Since this dissertation was focused on the transit buses, only a portion of the WVU test results was used. In the past two decades, WVU CAFEE has employed the TransLab and tested over 400 transit buses at different locations throughout the U.S, which could reflect the operations of major transit agencies in the country. For those transit buses, emission data from thousands of tests runs were collected, including repeat tests performed on specific buses. Over 25 different types of fuel were involved and 30 different test cycles were performed. Among them, major types of fuel used were diesel including D1, D2 and ULSD1 as well as CNG which were the focus of this study. Sixteen standard cycles have been primarily used in this study and their characteristics were introduced in Section 2.3 and 2.4.

The emission databases of WVU chassis dynamometer tests were stored on a CAFEE server following quality control after testing. For each test run, both continuous and integrated test data were available and have been employed in this study. Users could use a Visual Basic (VB) based software to query the second-by-second test data from the server. Continuous emission data were employed in this study to generate virtual cycles associated with their emission data tested on the same buses thus significantly expanded the limited data source for modeling.

For each test run, a short report could be obtained to show the summary of the tested vehicle parameters, emissions data, and any on-site issues involved. One short report with repeating runs is attached in Table A-1 of Appendix A as an example. Integrated emission data in the database was stored in a Microsoft Access database and now the bus database has been uploaded on the IBIS website as a searchable database accessible to the public. Figure 3.4 provides a screenshot of another query example from the online version of the searchable database [77]. Users can define selection criteria and query the dataset. Examples from the 
offline Access database on which the online searchable database was based and portion of the results are attached as Figure A-2 and Table A-3 respectively in Appendix A.

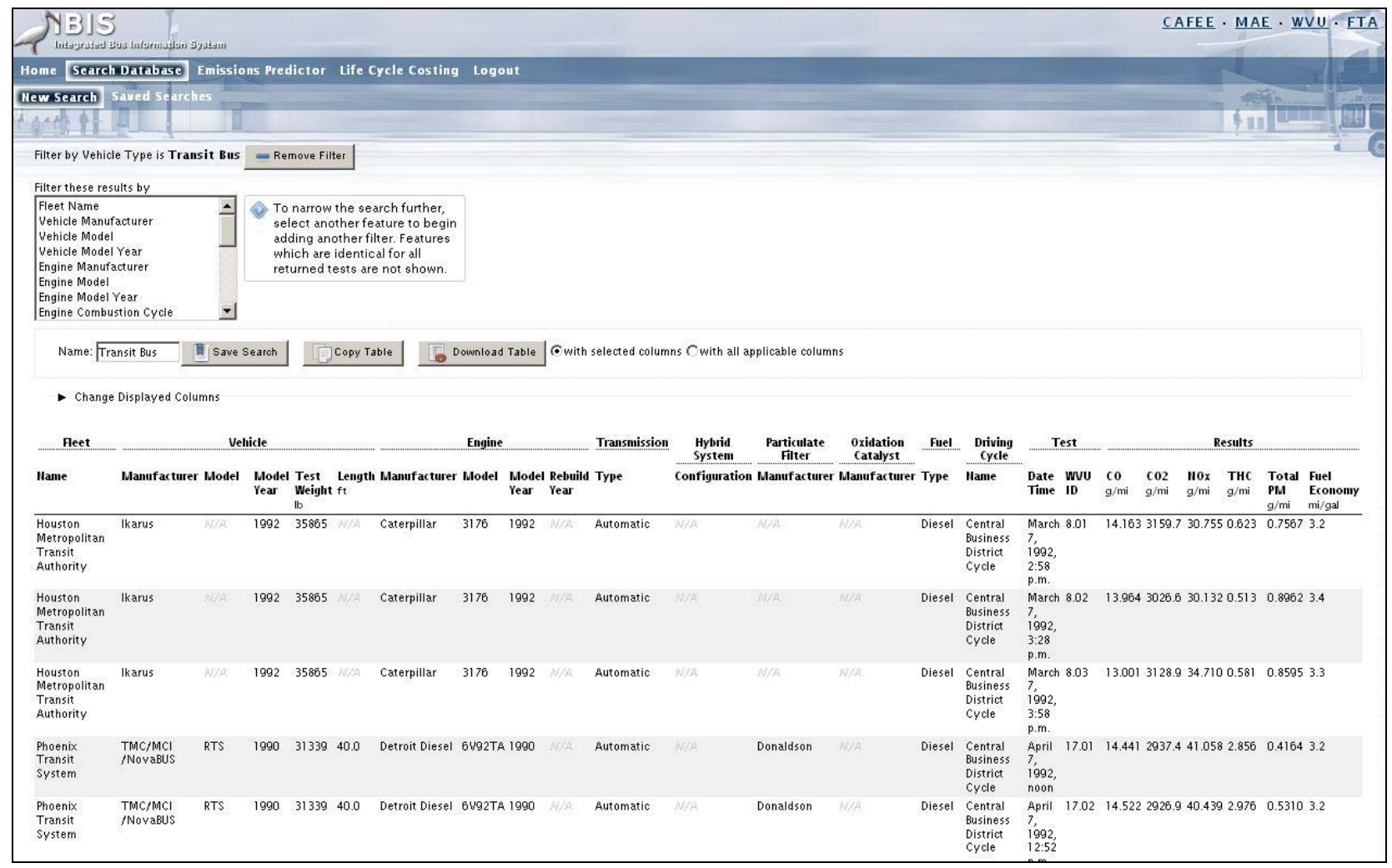

Figure 3.4 Screenshot of a Query Example from the Online Version of IBIS Searchable Bus Emissions Database 


\section{CORRELATION ANALYSIS OF DRIVING CHARACTERISTICS EFFECTS}

In this chapter, the correlation and regression analysis of the impact of duty cycle parameters (metrics) on emissions and fuel consumptions are discussed [84]. Based on these analyses, the input parameters for the cycle generation process and IBIS backbone model development were determined.

\subsection{Introduction}

The purpose of this study was to investigate the effects of cycle characteristics on distance-specific emissions of $\mathrm{CO}, \mathrm{CO}_{2}, \mathrm{NOx}, \mathrm{HC}, \mathrm{PM}$, and fuel economy in order to identify the most important parameters that should be included in the IBIS emissions model. This study was unique because WVU collected emissions data from multiple chassis dynamometer test cycles on the same vehicle [71]. Appropriate data interpolation was used to preliminarily expand a database so the relationships between cycle metrics and their impacts on emissions and fuel economy could be statistically investigated. In previous studies, data from only a limited number of test cycles on the same vehicle (typically five or less) were available which limited the effectiveness of the statistical analysis. This study identified the most influential cycle metrics for inclusion in the IBIS emissions model.

To encompass the multiple dimensions of duty cycle characteristics, thirteen cycle metrics were considered in this study: average speed with idle (or average speed) and without idle, number of stops per mile (stops/mile), percentage idle, standard deviation of speed with and without idle, average and maximum acceleration, average and maximum deceleration, aerodynamic speed, characteristic acceleration, and kinetic intensity [63]. A correlation analysis of these thirteen cycle metrics was performed to reduce dimensionality and remove bivariate collinearity. In selecting the metrics that would be used in the IBIS emissions model, the ability of transit agencies to compute the values of the metrics using data available to them was also considered. In some instances, metrics were retained or eliminated based on this additional criterion. Due to some non-linear relationships, a non-parametric correlation analysis was applied to determine the order of importance of the chosen metrics for each emissions and fuel economy prediction. Preliminary regression analysis was performed to demonstrate and reinforce 
the significant effect of the selected cycle metrics for modeling. JMP® statistical software [83] and MATLAB ${ }^{\circledR}[86]$ were used for data analysis as well as correlation and regressions analyses in this study.

\subsection{Test Vehicle}

The vehicle used in this analysis was backbone bus \#1 (listed in Table 6.1) which was a MY 2000 Orion diesel transit bus tested at the WMATA facility to compare effects of different cycles on emissions and fuel economy.

The bus had a gross vehicle weight rating (GVWR) of 42,540 lbs with the vehicle curb weight of 28,800 lbs. The weight as tested was 33,300 lbs, representing half-seated passenger load. The test bus was powered by a 2000 model year, 8.5 liter, 4-cylinder, 275 horsepower, Detroit Diesel S50 engine with a diesel oxidation catalyst (DOC). The fuel used by the bus was ULSD1. The vehicle was equipped with a 4-speed Voith D863 automatic transmission. The vehicle configuration was kept the same for all tests making the impact analysis exclusively for cycle characteristics.

The test vehicle was operated through twelve chassis dynamometer cycles for this study and multiple repeat runs of certain test cycles were performed. Totally thirteen cycle parameters were considered in this study and were shown in Table 2.4.

\subsection{Preliminarily Extended Database}

Since only 12 cycles were available for analysis, an expanded database was desired. Figure 4.1 shows CO emissions as a function of cycle average speed spanning from the lowest speed of 3.57 miles per hour (mph) (NYBUS cycle) to the highest speed of $43.72 \mathrm{mph}$ (COMM cycle). No test cycles existed between an average speed of 28.63 mph (ETC_12 cycle) and 43.72 mph (COMM cycle).

Interpolation was used to extend the database to fill the gaps as mentioned above. Initially, 18 cycle points were interpolated using an equal interval of two miles per hour for the average speed. A piecewise cubic hermite interpolating polynomial (pchip) [86-87] was based on the piecewise cubic interpolation and was applied in this study. A comparison with other interpolation methods is shown in Figure 4.1 with the assumption that no extreme cycle characteristics exist between adjacent cycle points. Compared with linear interpolation, pchip 
interpolation was smoother and less likely to overshoot. Although spline interpolation had smoother results than pchip, it was not considered because it caused more oscillation in data interpolation. To be noted, the piecewise curves were employed in this study to interpolate between data points rather than to fit them.

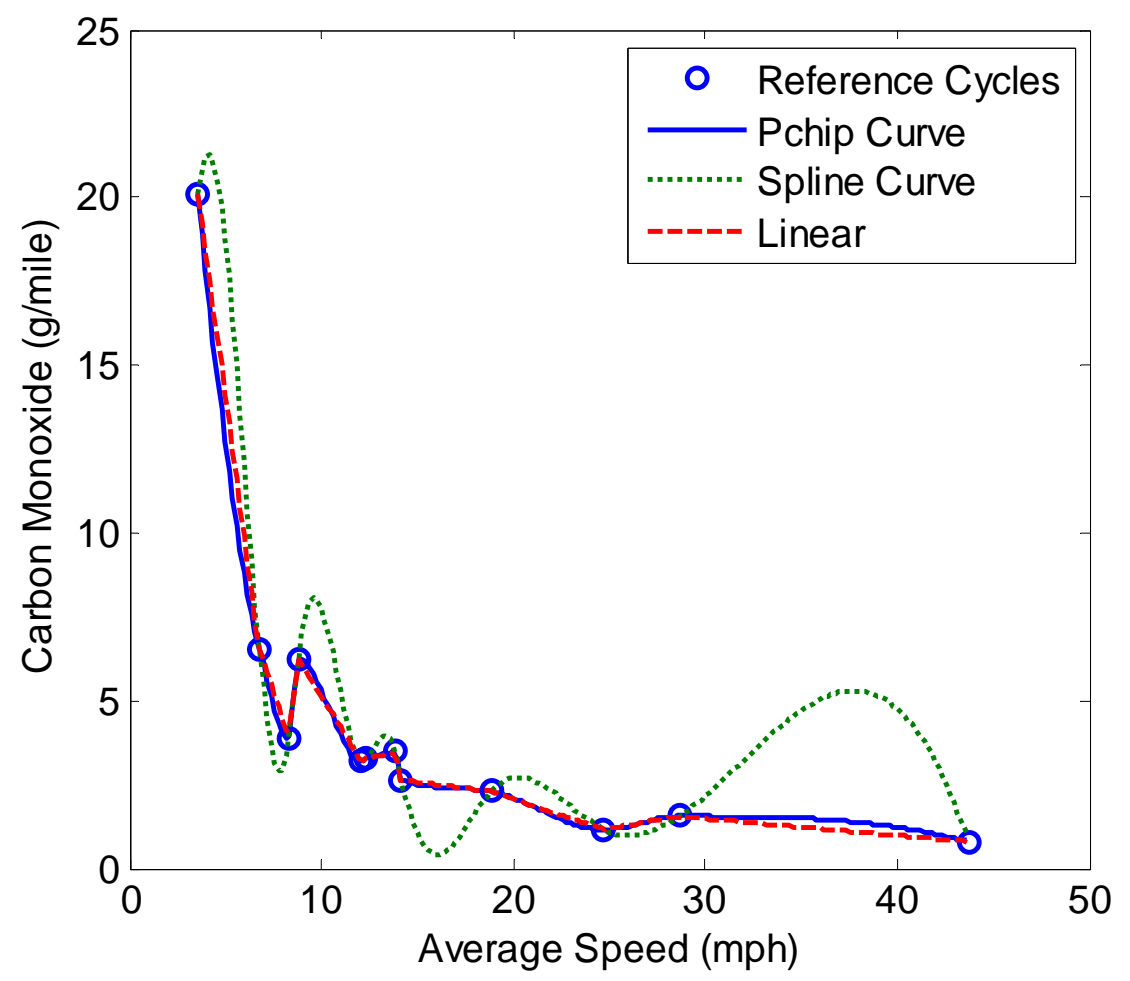

Figure 4.1 Reference Cycles and Comparison of Interpolation Curves Based on Average Speed

The same analysis and method were applied to the other four cycle metrics. The magnitudes of the intervals were $10 \%$ for percentage idle, four for stops per mile (stops/mile), three miles per hour for standard deviation of speed, and one reciprocal of unit mile ( $1 / \mathrm{mile})$ for kinetic intensity. In this way, 44 cycle points were generated to extend the database to 56 cycle points.

Figure 4.2 illustrates an example of the effectiveness of the interpolation by comparing the interpolation results in the plane spanned by kinetic intensity and average speed. The original and extended datasets were close to the exponential regression line with a coefficient of determination of 0.94 . When emissions and fuel economy data were plotted against duty cycle metrics, no significant deviation from the reference dataset was observed and the interpolated cycle points followed the same trend with the reference points. 


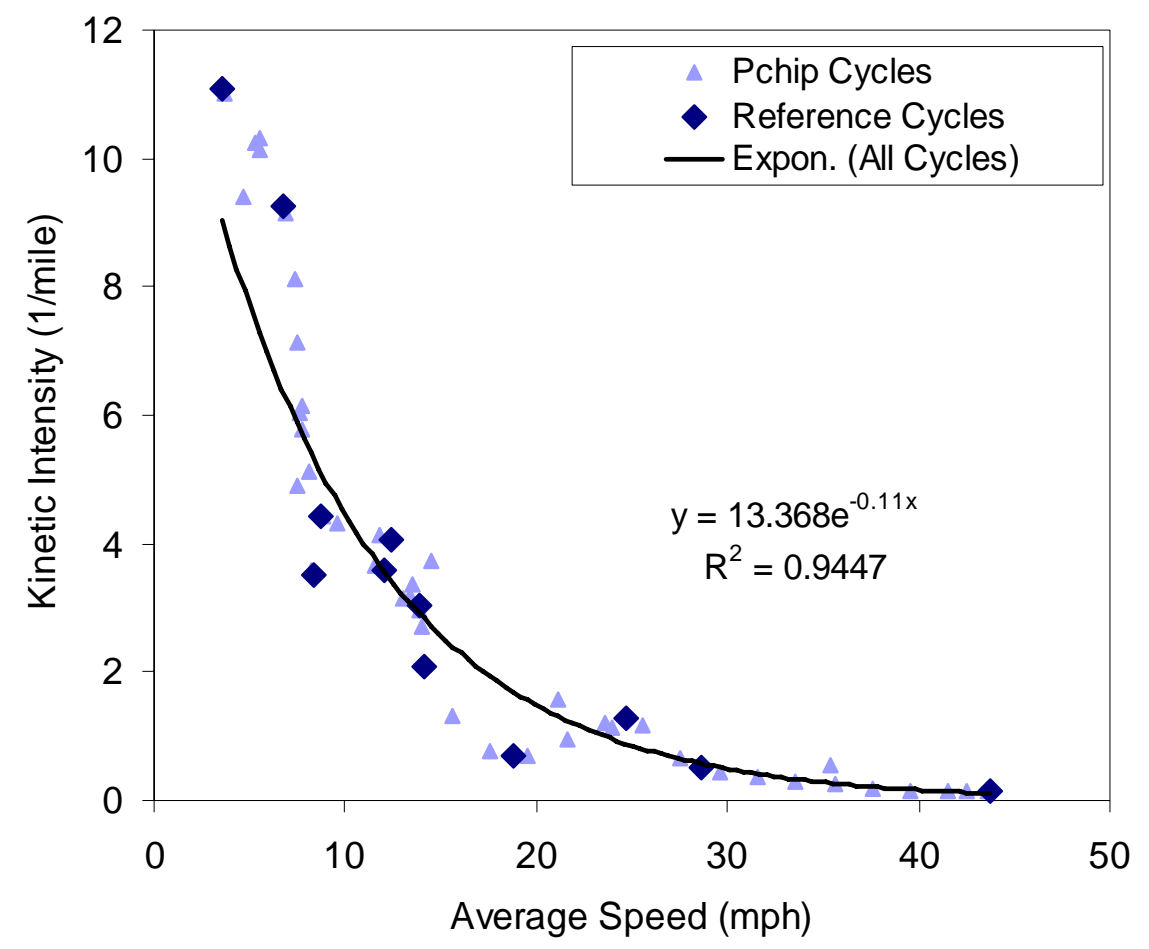

Figure 4.2 Comparison of Extended Dataset with Reference Cycles on the Plane of Kinetic Intensity vs. Average Speed

\subsection{Road Load Derived Cycle Metrics}

Section 2.4 briefly introduced three road load derived cycle metrics: aerodynamic speed, characteristic acceleration and kinetic intensity. Table 4.1 presents their correlations with distance-specific emissions and fuel economy.

Table 4.1 Correlations of Roadload Derived Cycle Metrics with Emissions and Fuel Economy

\begin{tabular}{lcccccc}
\hline & $\mathbf{C O}_{2}$ & CO & HC & NOx & PM & FuelEco \\
\hline AeroV & -0.77 & -0.70 & -0.80 & -0.66 & -0.72 & 0.85 \\
CharAcc & 0.89 & 0.78 & 0.79 & 0.82 & 0.81 & -0.94 \\
KInt & 0.94 & 0.89 & 0.93 & 0.87 & 0.90 & -0.84 \\
\hline
\end{tabular}

The negative correlations between aerodynamic speed and emissions indicated that emissions increased with decreasing aerodynamic speed, while the positive correlations showed that fuel economy increased along with increasing aerodynamic speed. However, the characteristic acceleration had an inverse correlation compared with that of aerodynamic speed, which made sense because larger characteristic acceleration required more kinetic energy to accelerate the vehicle indicating higher fuel consumption and increased emissions. Kinetic intensity combined both aerodynamic speed and characteristic acceleration and showed the same 
correlation trend with characteristic acceleration but had stronger correlations overall compared to the other two metrics.

Emissions and fuel economy were plotted against kinetic intensity as shown in Figure 4.3.
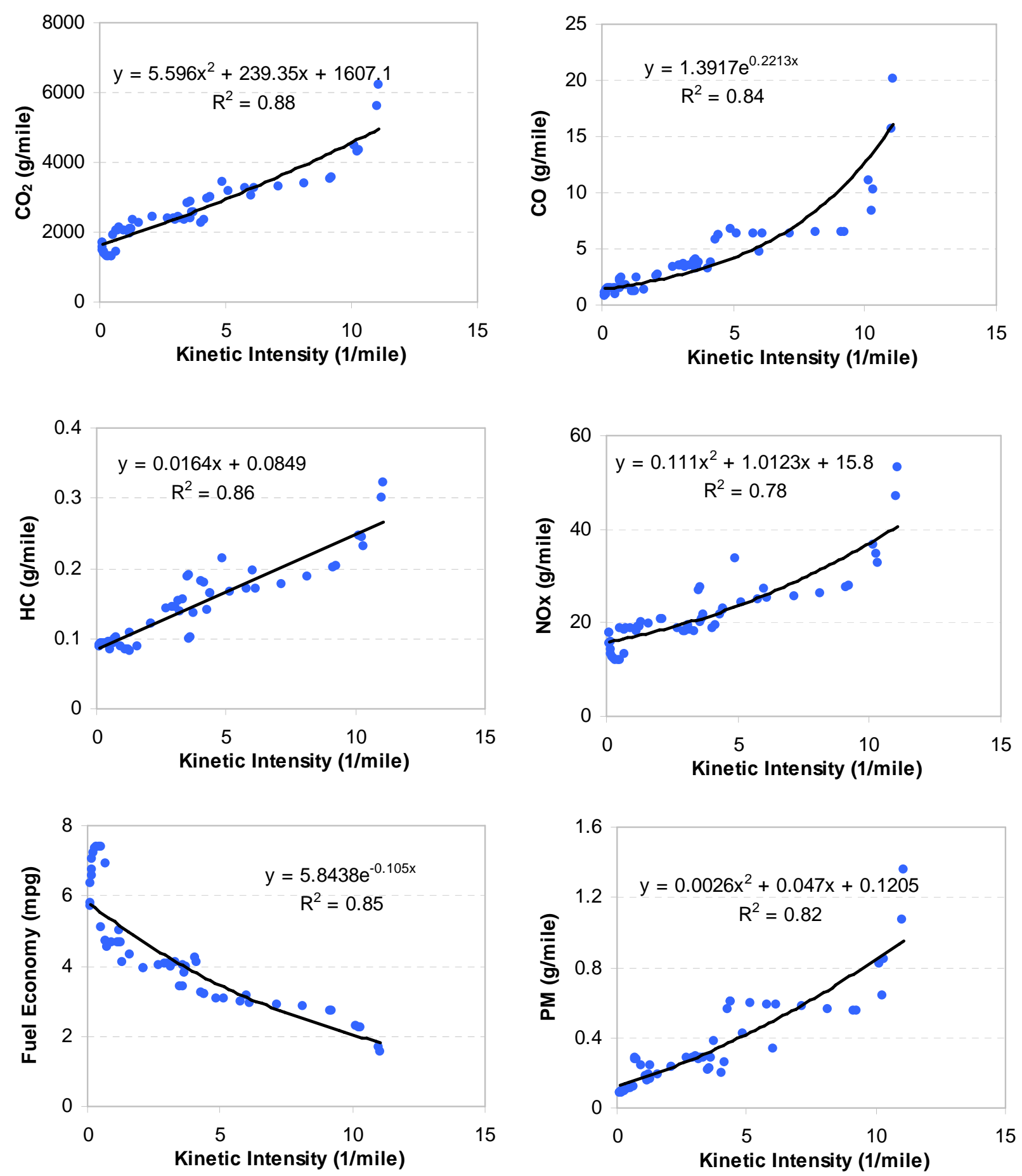

Figure 4.3 Emissions and Fuel Economy Against Kinetic Intensity 
It shows that $\mathrm{CO}_{2}$, NOx, and PM have second degree polynomial relationships with kinetic intensity. The $\mathrm{CO}$ emissions and fuel economy showed exponential relationships while HC had a linear trend. Their $R$ squared ranged between 0.82 and 0.89 with the exception of 0.78 for NOx. If more data were available for analysis, stronger relationships could be expected. These results indicated the significant impact of kinetic intensity on emissions and fuel economy and their relationships might not be linear.

\subsection{Correlation Analysis}

A Pearson correlation matrix was applied to detect bivariate collinearity among the cycle parameters. The analysis showed that several variables highly correlated with each other. The existence of collinearity was not a violation of the assumptions for regression analysis. However, it indicated that several cycle metrics had similar impact on emissions and fuel economy, and could potentially be removed from the analysis as redundant. Collinearity also makes it difficult to interpret the partial regression coefficients, which measure the effect of the corresponding cycle metrics while holding constant all other metrics. When collinearity exists, those coefficients estimate some effects for the response, which are not really from corresponding metrics. Table 4.2 shows full correlation coefficients with significance levels for all thirteen cycle metrics.

Statistically significant and strong correlation pairs were found in the following cases:

- Average speed with idle versus average speed without idle, aerodynamic speed and characteristic acceleration;

- Average speed without idle versus standard deviation of vehicle speed with idle and aerodynamic speed;

- Stops per mile versus percentage idle and kinetic intensity;

- Standard deviation of vehicle speed with idle versus aerodynamic speed and standard deviation of vehicle speed without idle. 


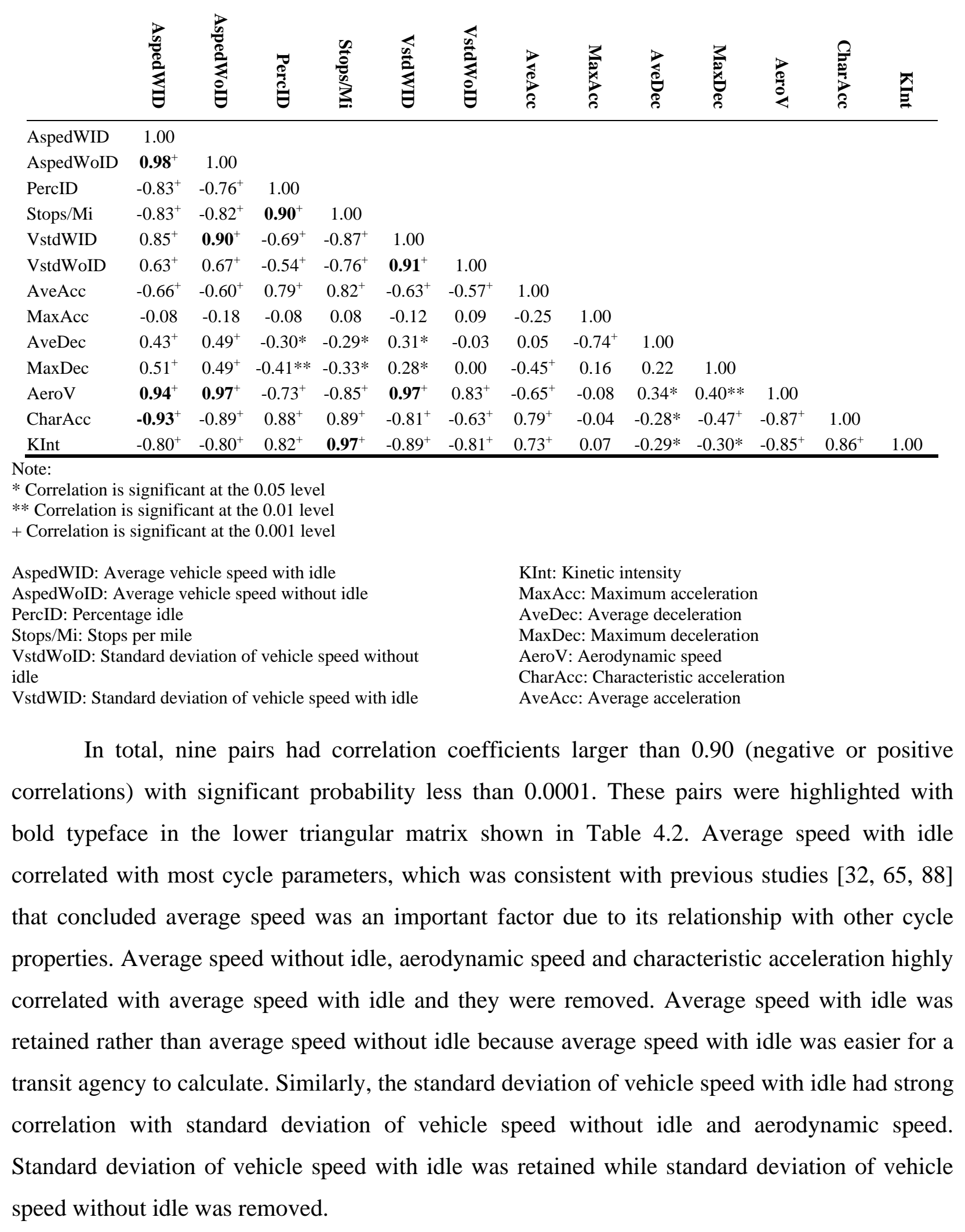


Aerodynamic speed correlated with both average speed and the standard deviation of vehicle speed, indicating that it might reflect the statistical features of vehicle speed such as mean and dispersion. However, aerodynamic speed was removed, because average speed and standard deviation of vehicle speed were retained. Additionally, Equation 2.4 shows kinetic intensity related to both aerodynamic speed and characteristic acceleration. Figure 4.3 also implies the importance of kinetic intensity to emissions and fuel economy. Thus, it was better to retain kinetic intensity instead of aerodynamic speed or characteristic acceleration.

The number of stops per mile might reflect the transient nature of driving cycles and it was readily acquired, thus the metric was retained as was percentage idle because of their impact on emissions [71], although both metrics strongly correlated with each other. However, this strong positive correlation could not be well explained. For example, more stops in a trip do not necessarily mean higher percentage idle. If a shorter idle duration occurs at each stop, total idle time of that trip can be less than that of a trip with a longer idle duration at each stop and fewer total stops during the trip. The strong correlation between kinetic intensity and stops per mile indicated that both metrics reflected some features of the transient driving behavior.

Certain redundant metrics were retained because they could be easily determined from basic route information available to transit agencies. Retention of these cycle metrics resulted in collinearity, however, a potential predictive model did not necessarily have to include all selected cycle metrics as explanatory variables. After some collinearity was removed, the total number of metrics decreased from thirteen to nine.

It is evident from Table 4.2 that the four cycle parameters including AveAcc, MaxAcc, AveDec and MaxDec have weak correlations with the other cycle parameters. To be useful for emissions modeling, they should correlate with emissions and fuel economy. Table 4.3 shows the correlations with significance levels of the four parameters with emissions and fuel economy. Average acceleration showed moderate correlations at the 0.001 significance level while maximum acceleration, average deceleration, and maximum deceleration were not well correlated with emissions and fuel economy.

The effects of average deceleration were less significant than that of average acceleration. This might be explained that during deceleration the engine is often at idle indicating weak contribution of deceleration to emissions and fuel consumption. However, when a vehicle accelerates, more fuel is consumed, producing more emissions [89]. It was also noted that 
maximum acceleration and deceleration did not correlate with emissions and fuel economy, which might be due to both metrics corresponding to single points in the entire cycle. Based on the above analysis, average deceleration, maximum acceleration, and maximum deceleration were removed from further consideration.

Table 4.3 Correlations with Significance Levels of Four Cycle Metrics vs. Emissions and Fuel Economy

\begin{tabular}{lcccccc}
\hline & $\mathbf{C O}_{2}$ & CO & HC & NOx & PM & FuelEco \\
\hline AveAcc & $0.84^{+}$ & $0.81^{+}$ & $0.79^{+}$ & $0.84^{+}$ & $0.77^{+}$ & $-0.76^{+}$ \\
MaxAcc & 0.02 & 0.18 & 0.05 & -0.09 & 0.24 & 0.14 \\
AveDec & -0.25 & $-0.33^{*}$ & $-0.31^{*}$ & -0.18 & $-0.33^{*}$ & 0.15 \\
MaxDec & $-0.32^{*}$ & $-0.27^{*}$ & $-0.30^{*}$ & $-0.34^{*}$ & -0.22 & $0.30^{*}$ \\
\hline
\end{tabular}

Note:

* Correlation is significant at the 0.05 level

+ Correlation is significant at the 0.001 level

FuelEco: Fuel economy

AveAcc: Average acceleration

MaxAcc: Maximum acceleration

Through the initial correlation analysis of thirteen cycle parameters, six parameters were identified most important and useful for evaluation of emissions and fuel economy, and seven parameters were removed because they were either redundant or appeared to have little correlation with emissions and fuel economy. The six cycle parameters selected were average speed with idle, percentage idle, stops/mile, standard deviation of vehicle speed with idle, kinetic intensity and average acceleration.

\subsection{Non-parametric Correlation Analysis}

As previously mentioned, if a nonlinear relationship actually exists between paired variables, classic Pearson's correlation $R$ will underestimate their relationship. For example, in this study the Pearson's correlation between $\mathrm{CO}_{2}$ and average speed was -0.78 with a coefficient of determination of 0.60 . Actually, both variables had a power decay relationship as shown in Figure 4.4 exhibiting a much better fit with an $R$ squared of 0.91 . Considering this, the nonparametric statistical correlation - the Spearman's correlation $\rho$ was used to more accurately evaluate the relationship. 


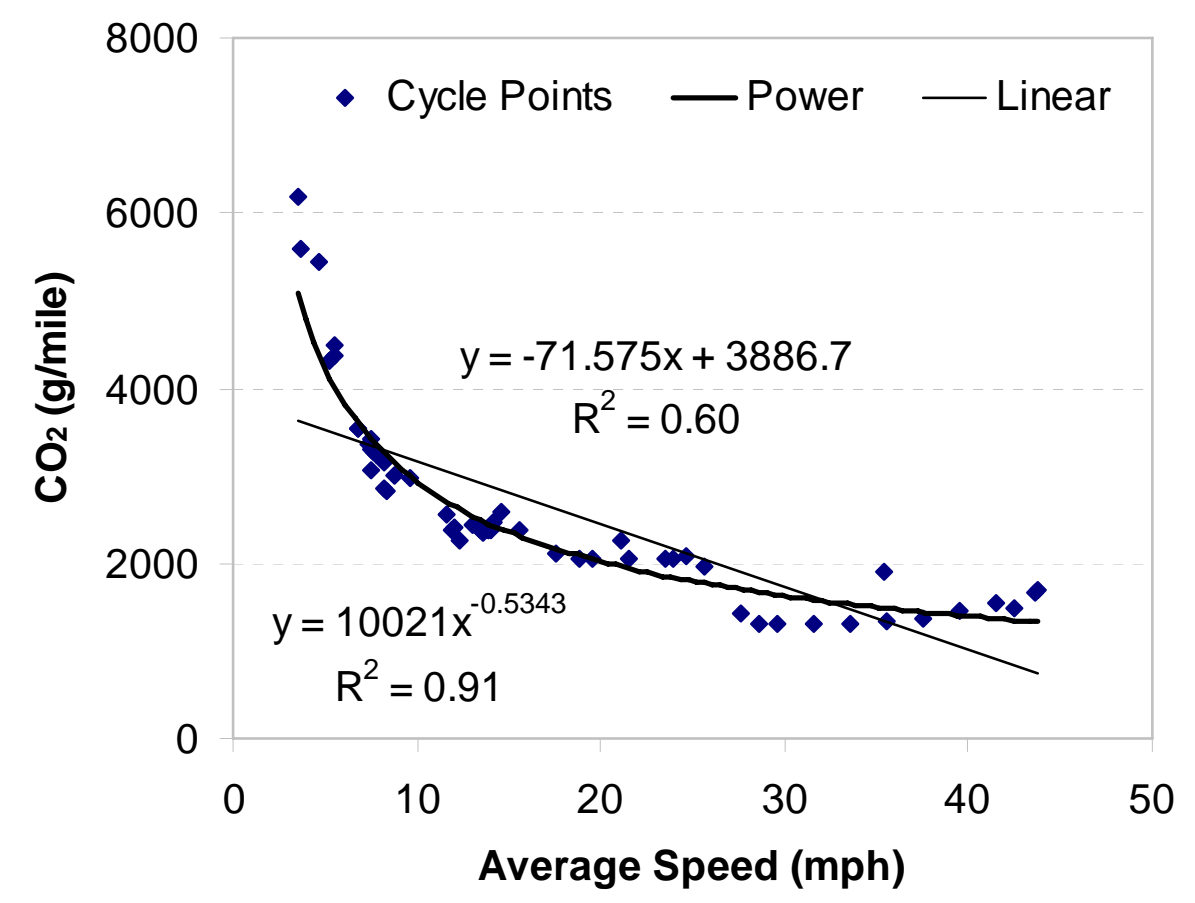

Figure 4.4 $\mathrm{CO}_{2}$ Emission as a Function of Average Speed to Show Non-linear Relationship

The Spearman's correlation $\rho$ is a rank correlation of the data and it does not require variables to be normally distributed nor linear. The meaning and range of $\rho$ are essentially the same as that of $R$. Zero represents no correlation; 1 or -1 indicates the perfect positive or negative fit; $\rho$ between 0 and 1 means increasing $X$ corresponds to increasing $Y$ and vice versa; $\rho$ between 0 and -1 means increasing $X$ corresponds to decreasing $Y$ and vice versa.

The Spearman's correlations of the six selected cycle metrics with emissions and fuel economy are shown in Table 4.4 all with statistically significant probabilities of less than 0.0001 . Average acceleration had the weakest correlation, making it the least important among the six selected metrics. The detailed analysis for the importance of the other five metrics is listed as follows.

$\mathrm{CO}_{2}$ Emissions. The $\mathrm{CO}_{2}$ emissions had the strongest correlation with average speed with a coefficient of -0.9546 , indicating that higher vehicle average speed resulted in lower $\mathrm{CO}_{2}$ emissions. Actually, all other emissions had negative correlations with average speed. This showed that higher average speed produced lower emissions, which was consistent with previous findings [71]. Higher vehicle average speed usually involves fewer accelerations and decelerations, resulting in lower emissions. Stops per mile had the second largest correlation of 0.9540 with $\mathrm{CO}_{2}$ followed by kinetic intensity with a correlation of 0.9537 . Positive correlations 
implied that more stops per mile and higher kinetic intensity produced higher $\mathrm{CO}_{2}$ emissions. Since the values of these three correlations were very close to each other, it was difficult to tell which metric was most important for $\mathrm{CO}_{2}$ emissions. Percentage idle and standard deviation of vehicle speed had correlations of 0.91 and -0.87 with $\mathrm{CO}_{2}$ emissions, respectively. The negative correlation showed $\mathrm{CO}_{2}$ decreased with increased standard deviation of vehicle speed. However, at the same average speed, increased standard deviation usually implies more transient cycle features which would produce higher $\mathrm{CO}_{2}$.

Table 4.4 Non-parametric Spearman's Correlation with Significance Levels

\begin{tabular}{lcccccc}
\hline & $\mathbf{C O}_{2}$ & CO & HC & NOx & PM & FuelEco \\
\hline AspedWID & -0.9546 & -0.965 & -0.9208 & -0.908 & -0.9131 & 0.9558 \\
PercID & 0.9144 & 0.8674 & 0.8321 & 0.9172 & 0.8552 & -0.9055 \\
Stops/Mi & 0.954 & 0.9665 & 0.9134 & 0.9033 & 0.9339 & -0.9528 \\
VstdWID & -0.8676 & -0.8917 & -0.8634 & -0.8015 & -0.8014 & 0.8729 \\
AveAcc & 0.6309 & 0.5441 & 0.5466 & 0.5833 & 0.5871 & -0.6252 \\
KInt & 0.9537 & 0.9423 & 0.877 & 0.9032 & 0.9183 & -0.9534 \\
\hline
\end{tabular}

Note: All correlations are significant at the 0.0001 level $(\mathrm{p}<0.0001)$

FuelEco: Fuel economy

AspedWID: Average vehicle speed with idle

KInt: Kinetic intensity

PercID: Percentage idle

Stops/Mi: Stops per mile

VstdWID: Standard deviation of vehicle speed with idle

AeroV: Aerodynamic speed

CharAcc: Characteristic acceleration

AveAcc: Average acceleration

CO Emissions. For CO emissions, stops per mile had the strongest positive correlation of 0.9665, which was reasonable since CO emissions in gram per mile were sensitive to the transient features of driving activities [65]. The more stop-and-go features, the more deviations there were from steady state, and the higher CO emissions that were produced. Average speed had the second strongest correlation of -0.965 and kinetic intensity had a correlation of 0.942 .

HC Emissions. HC emissions had the strongest correlation of 0.92 with average speed, followed by stops per mile of 0.91 . The other correlations were below 0.9 , indicating that stops per mile and average speed were the two most important metrics for HC emissions.

NOx Emissions. NOx emissions showed the strongest correlation with percentage idle, which was consistent with the fact that excessive idling could produce more NOx emissions [65]. It was also noticed that average speed, stops per mile, and kinetic intensity had strong correlations of 0.90 and above with NOx, indicating their significance in this type of emissions.

PM Emissions. PM showed the strongest correlation of 0.93 with stops per mile. PM was also highly correlated with CO (0.92), reinforcing that both CO and PM were sensitive to the 
transient features of driving activities. In addition, PM had strong correlations with average speed and kinetic intensity and both were above 0.90 .

Fuel Economy. Fuel economy strongly correlated with average speed with a correlation coefficient of 0.96, indicating the higher the average speed the lower the amount of fuel consumed. To be noted, it does not mean this trend would be consistent at much higher average speed. Previous studies showed the fuel economy would reach the maximum at a specific vehicle speed and would decrease at higher average speeds as the aerodynamic drag begins to dominate. The result was a parabolic curve [70, 71].

The order of significance of the six cycle metrics' impact on emissions and fuel economy is summarized in Table 4.5. The strong, moderate and weak correlations were defined as coefficients higher than 0.90 , between 0.80 and 0.90 , and below 0.80 , respectively. Stops per mile and average speed had strong correlations with all emissions and fuel economy. This result was consistent with the common interpretation that average speed reflected cruise features of driving activities while stops per mile was linked to transient features. Emissions and fuel economy might reflect effects from both cruise and transient features of the driving cycles. However, it was difficult to tell which metric was the most important one, because metrics in the strong correlation category showed very similar correlation coefficients.

Table 4.5 Summary of Order of Importance for the Selected Six Cycle Parameters

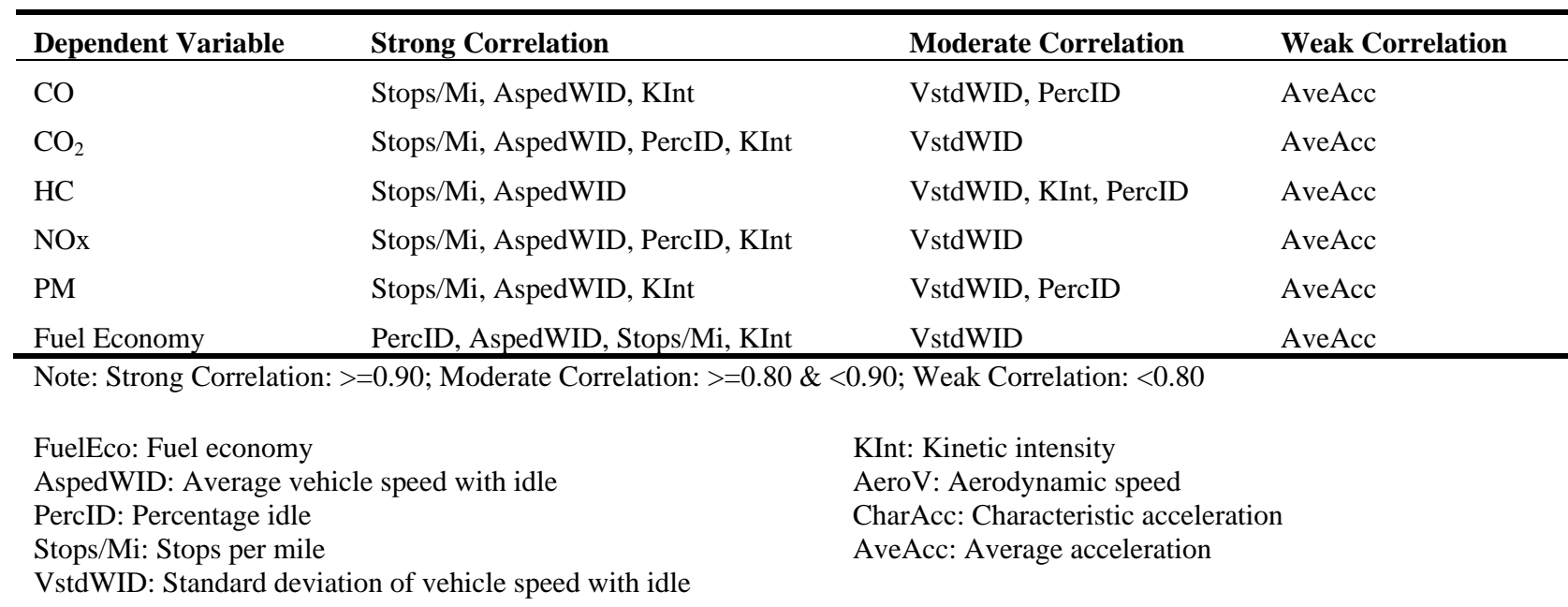

\subsection{Preliminary Regression Analysis}

To validate the significant effects of selected cycle parameters on emissions and fuel economy, preliminary regression analysis was performed with selected parameters as 
independent variables. The results were compared with average speed based regressions. The general regression model based on selected parameters was expressed as Equation 4.1.

$$
y=a+\sum_{i=1}^{5} b_{i} x_{i}+\sum_{i=1}^{5} c_{i} x_{i}^{2}+\varepsilon
$$

Equation 4.1

where $a$ is intercept, $b_{i}$, and $c_{i}$ are regression coefficients, and $\varepsilon$ is the residual term; $y$ is the dependent variable corresponding to emissions or fuel economy while $x_{i}$ are the independent variables corresponding to the five selected cycle parameters. Average acceleration was not included in this analysis due to its weak correlation with the dependent variables. The least square method was used to estimate the parameters in the multiple regression models. The squared term for each of the selected cycle parameters was added in the models to account for possible nonlinear relationships.

The results were compared with average speed based regressions as shown in Table 4.6. For each response variable, average speed based power regressions presented overall better fitting with significant coefficients compared to linear, polynomial, exponential and logarithmic regressions. Linear regressions were used to obtain the coefficients after the natural logarithm transformation was applied to response and explanatory variables. All $R$ squared values were greater than 0.85 except for 0.79 for NOx emissions and coefficients for the explanatory variable were significant at the 0.0001 probability level $(p<0.0001)$.

To demonstrate the significant effect of the selected cycle parameters, preliminary regression analysis was performed and the coefficients of multiple parameter models are shown in Table 4.7. The stepwise procedure was employed to select significant independent variables for the regression models. Compared to average speed based regressions in Table 4.6, the multiple metrics regressions in Table 4.7 show adjusted $R$ squared values above 0.95 except for 0.94 for PM, which is good considering the transient dependency of PM emissions. Most of RMSE values were substantially reduced (about half or more) except that of PM emissions. 
Table 4.6 Average Speed Based Regressions

\begin{tabular}{llll}
\hline Response & Regression & $\mathbf{R}^{2}$ & RMSE \\
\hline $\mathrm{CO}_{2}$ & $\mathrm{y}=10021 \mathrm{x}^{-0.5343}$ & 0.91 & 306.74 \\
$\mathrm{CO}$ & $\mathrm{y}=64.976 \mathrm{x}^{-1.147}$ & 0.94 & 1.18 \\
$\mathrm{HC}$ & $\mathrm{y}=0.5402 \mathrm{x}^{-0.5258}$ & 0.86 & 0.02 \\
NOx & $\mathrm{y}=66.8501 \mathrm{x}^{-0.4366}$ & 0.79 & 3.93 \\
Fuel Economy & $\mathrm{y}=0.9816 \mathrm{x}^{0.5298}$ & 0.91 & 0.60 \\
PM & $\mathrm{y}=4.1171 \mathrm{x}^{-1.0262}$ & 0.90 & 0.10 \\
\hline
\end{tabular}

Table 4.7 Regression Models Based on Selected Parameters

\begin{tabular}{|c|c|c|c|c|c|c|}
\hline Term & $\mathrm{CO}_{2}$ & CO & HC & NOx & Fuel Economy & PM \\
\hline Intercept & 507.715 & -0.017 & $0.193 *^{+}$ & 3.236 & $6.730 *^{+}$ & $-0.207 *$ \\
\hline AspedWID & $15.492 * *$ & - & - & $0.276^{*^{+}}$ & $-0.046^{+}$ & - \\
\hline PercID & $3268.232 *^{+}$ & - & $0.138 *^{+}$ & $46.742 *^{+}$ & $-9.523 *^{+}$ & - \\
\hline (PercID-0.268)*(PercID-0.268) & $-6125.302 *^{+}$ & - & $0.426 *^{+}$ & - & $31.291 *^{+}$ & - \\
\hline Stops/Mi & $111.860 * *$ & $0.673 *^{+}$ & - & 0.286 & -0.116 & $0.068 *^{+}$ \\
\hline (Stops/Mi-5.20683)*(Stops/Mi-5.20683) & $12.603^{*^{+}}$ & $0.068^{*^{+}}$ & - & $0.069 *^{+}$ & $-0.017 * *$ & $0.001 * *$ \\
\hline VstdWID & 17.135 & - & $-0.008^{+}$ & -0.132 & 0.060 & $0.014 *$ \\
\hline (VstdWID-12.8037)*(VstdWID-12.8037) & $-11.253^{*^{+}}$ & - & $0.001^{*^{+}}$ & $-0.070 * *$ & $0.021 *^{+}$ & - \\
\hline KInt & $73.522^{+}$ & 0.052 & - & $0.508^{*}$ & - & - \\
\hline$($ KInt-3.58075)*(KInt-3.58075) & - & $-0.060^{+}$ & - & - & - & - \\
\hline Adjusted $\mathrm{R}^{2}$ & 0.99 & 0.98 & 0.96 & 0.98 & 0.98 & 0.94 \\
\hline RMSE & 86.15 & 0.52 & 0.01 & 1.07 & 0.22 & 0.07 \\
\hline
\end{tabular}

Note:

* Significant at the 0.05 level

** Significant at the 0.01 level

+ Significant at the 0.001 level

*+ Significant at the 0.0001 level

FuelEco: Fuel economy AspedWID: Average vehicle speed with idle PercID: Percentage idle

Stops/Mi: Stops per mile

VstdWID: Standard deviation of vehicle speed with idle

KInt: Kinetic intensity

AeroV: Aerodynamic speed

CharAcc: Characteristic acceleration 
Figure 4.5 compares the estimated and experimental values of emissions and fuel economy for the NYBus cycle based on the single parameter regressions (based on average speed) as shown in Table 4.6 and the multiple parameter regressions (based on selected parameters) as shown in Table 4.7. Figure 4.6 shows the percentage errors using experimental data from the NYBus cycle for both models. For the NYBus cycle, the multiple parameter models showed over 75\% smaller percentage errors for all responses, demonstrating significant improvement in predicting all responses. Figure 4.7 compares the mean percentage errors (MPE) using both models after considering all cycle points. It shows that on average multiple parameter models have more than 40\% reduction in MPE for $\mathrm{CO}_{2}$, $\mathrm{HC}$ and fuel economy. CO and PM had mean errors above $15 \%$ for both models, further indicating it was difficult to predict them due to their high sensitivity to transient features of vehicle operation. If interaction terms of the selected cycle metrics or the appropriate transformations (such as Box-Cox method) of response variables were considered in the analysis, the multiple parameter models might show further improvement.

The regression models developed herein were used to determine the impact of cycle metrics on emissions and fuel economy and to select cycle metrics for the development of a transit fleet emission model for the IBIS. The emissions model development and validation were presented in Chapter 6.

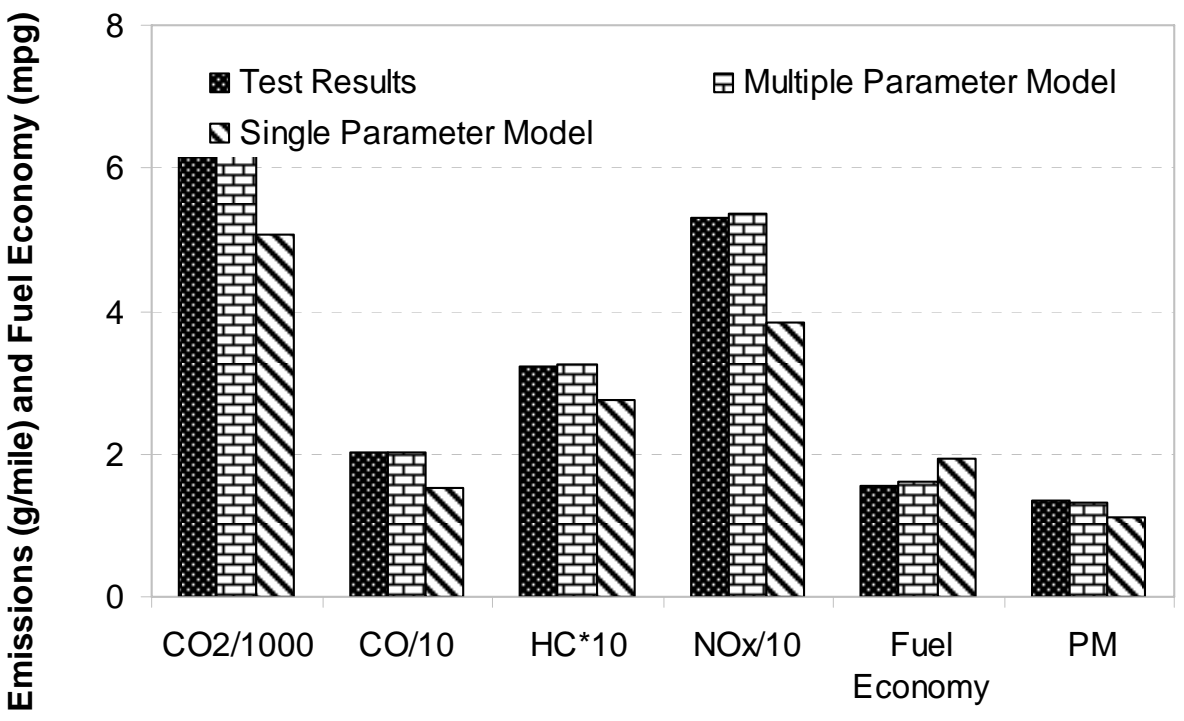

Figure 4.5 Comparison of Single and Multiple Parameter Models to Experimental Results for the NYBus Cycle 


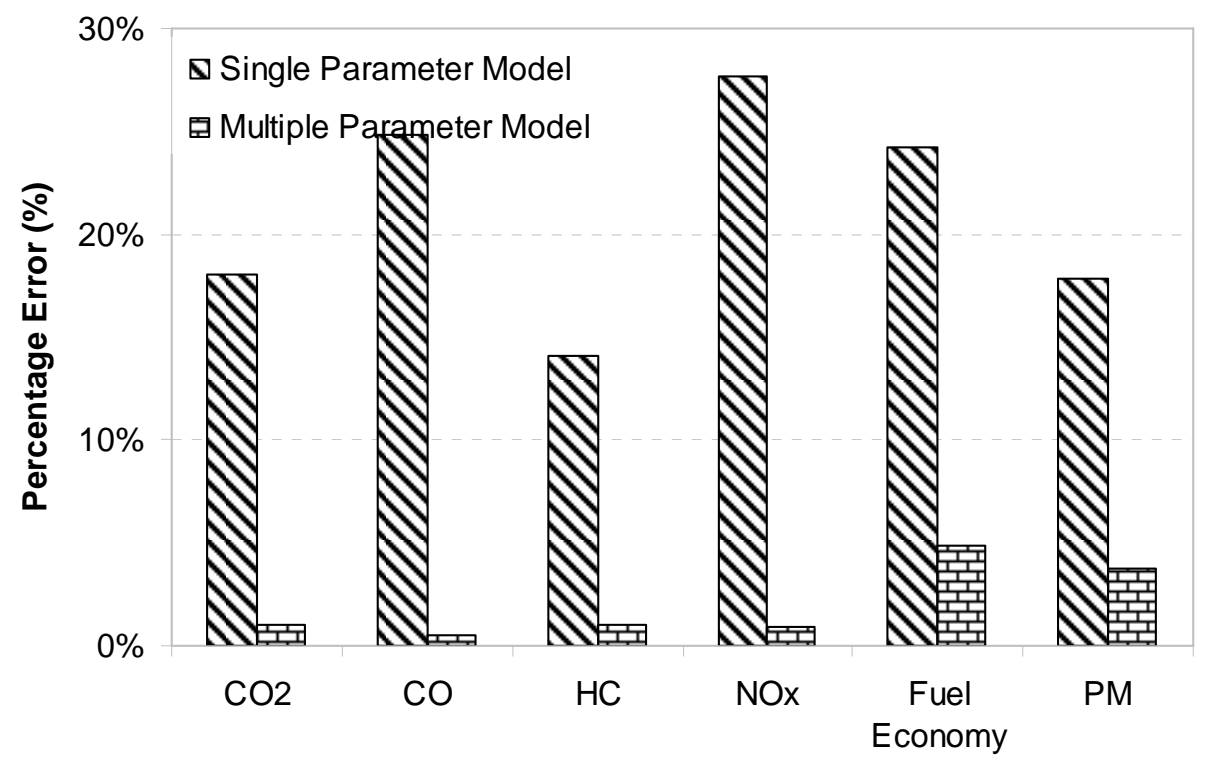

Figure 4.6 Estimation Errors of Single and Multiple Parameter Models for the NYBus Cycle

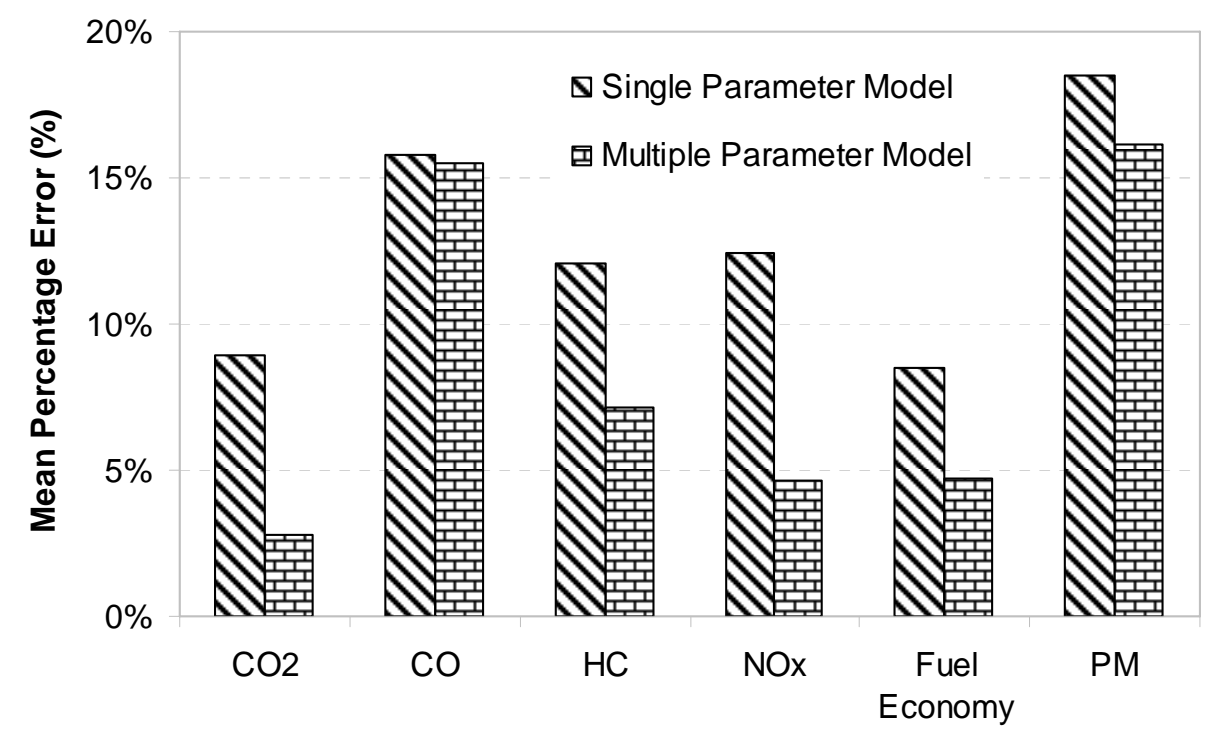

Figure 4.7 Mean Percentage Error Comparison between Single and Multiple Parameter Models

\subsection{Summary}

A detailed correlation analysis was performed to investigate the relationships between duty cycle metrics and emissions and fuel economy and to identify the most important parameters for modeling. Through the initial full correlation analysis among 13 cycle metrics, the number of metrics considered most useful for modeling was reduced to six. They were average speed with idle, percentage idle, stops per mile, standard deviation of vehicle speed, kinetic intensity and average acceleration. 
Further analysis using non-parametric Spearman's correlations between the six selected cycle metrics with each emission and fuel economy, showed that average acceleration had the weakest correlation, implying that its ability to predict emissions and fuel economy was less significant. The preliminary regression analysis demonstrated how adding selected cycle metrics to single parameter (average speed) models improved the regression models. The results of this study assisted in determining appropriate strategies for later backbone model development and implementation of IBIS transit fleet emissions model.

This study indicated that duty cycles had significant impacts on emissions and fuel economy of transit buses and it provided a useful framework for the selection of the most influential cycle metrics for modeling. In addition to the average speed, other cycle metrics such as stops per mile, percentage idle, standard deviation of vehicle speed and kinetic intensity were found to be important and could be used for a better prediction of emissions and fuel economy. In the interest of a green environment and energy efficiency, this study also suggested that if vehicles could operate less aggressively, spending more time in cruise mode, having less stopand-go patterns, or less idling behavior while parking, exhaust emissions and fuel consumption from the transportation sector would be reduced. As a result, air quality and energy efficiency would be improved. 


\section{GENERATION OF NEW VIRTUAL CYCLES WITH EMISSIONS DATA AND PRELIMINARY EXPERIMENTAL VALIDATION}

\subsection{Introduction}

Previous studies using emission data from multiple test cycles showed that distancespecific emissions profoundly depended on the characteristics of the duty cycles used [57-59, 64, 68]. However, due to the limited emission data from chassis dynamometer tests, to develop emission predictive models, more emission data from more testing cycles were needed to reflect the real operations of transit fleets. It was impractical to perform numerous chassis dynamometer tests due to the high cost and extensive effort involved. In this study, research effort was motivated to develop a novel virtual cycle generator to create sufficient cycles to fill sparse areas in the cycle parameter spaces as shown in Figure 5.1.

More importantly, with the assistance of the emission generator, a large amount of emissions and fuel economy data from the buses tested on the limited cycles could be generated for new virtual cycles. Consequently, the database for emission modeling could be improved with new data generated through this approach.

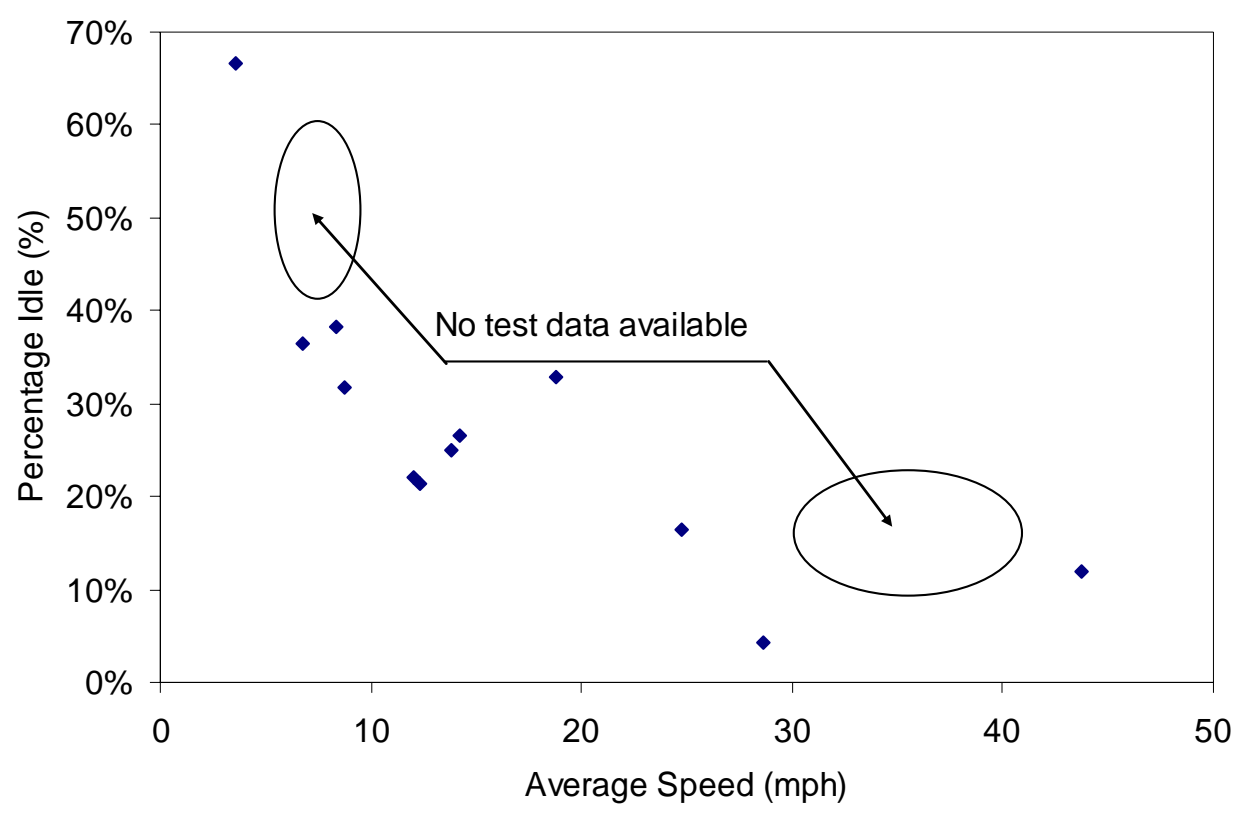

Figure 5.1 Sparse Areas on Cycle Parameter Planes 
Compared with the previous studies, the cycle generator in this study showed some differences from the followings. 1). Purpose of cycle generation. The previous cycle generators usually created one best cycle for emission characterization of local transit operations. However, the cycle generator in this study was used to create numerous virtual cycles to investigate the possible operation profiles of transit buses. Most importantly, if limited emission testing data was available abundant additional emission and fuel economy data could be produced with the new virtual cycles using the emission generator. As a result, databases for emission modeling could be dramatically improved. 2). Searching methodology. The Genetic Algorithm (GA) was implemented in the generator for more effective and comprehensive space search compared to random search method employed by previous studies which took longer searching for a desired candidate. 3). Microtrip source. In this study microtrips were extracted from twelve existing standard (target) test cycles while in previous studies microtrips were drawn from the actual vehicle activity database. 4). Input parameters. In this study, the number of inputs was not fixed and users could customize the weight and error for each input desired.

\subsection{Methodology}

New virtual cycles can be generated by selecting and reassembling the microtrips drawn from continuous speed-time traces of existing target duty cycles. An idle period was defined as vehicle speed below $0.5 \mathrm{mph}$. A microtrip was defined as a period starting with vehicle speed higher than $0.5 \mathrm{mph}$ and ending with vehicle speed lower than $0.5 \mathrm{mph}$ plus the next idle period. A typical microtrip is shown in Figure 5.2. 


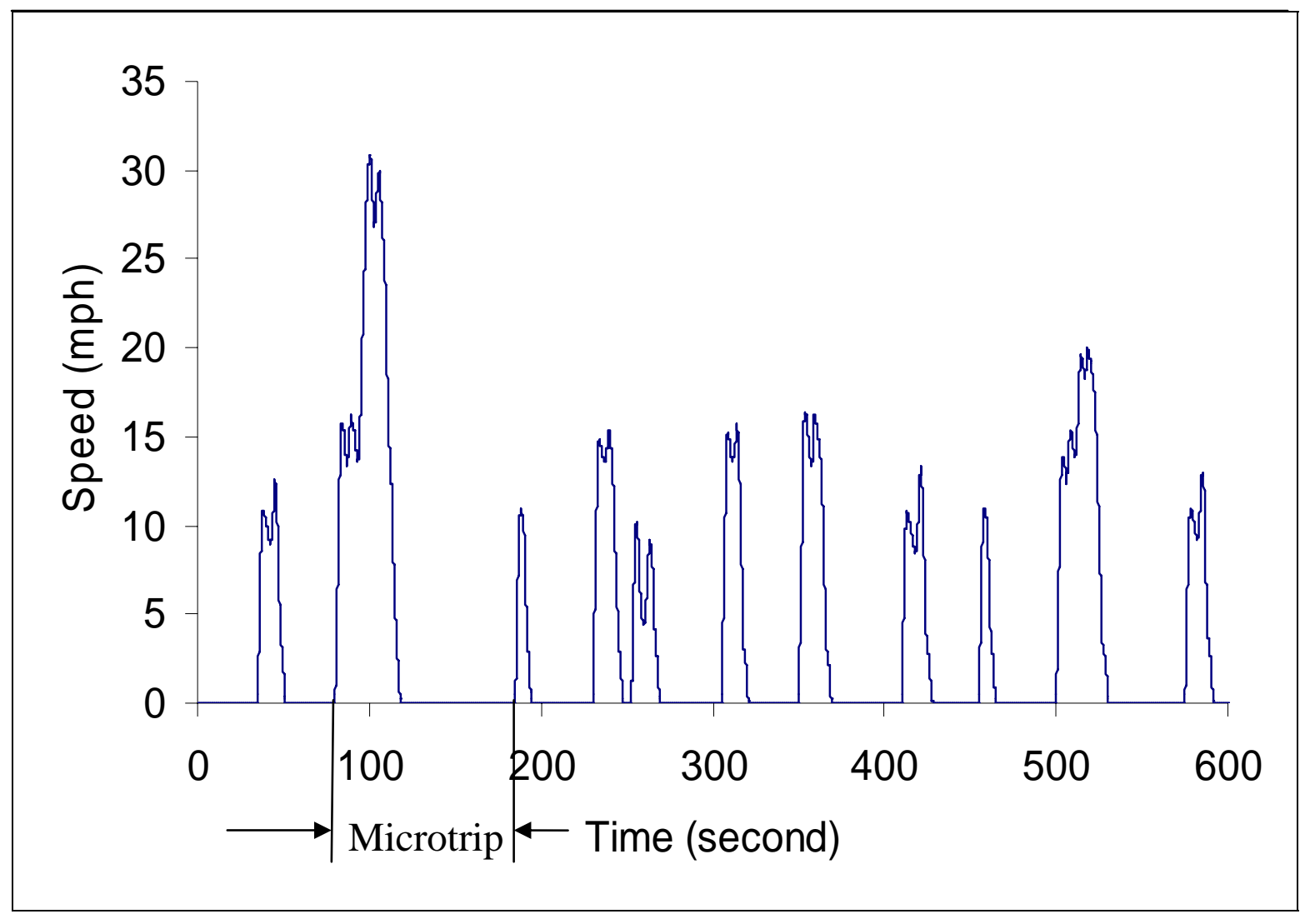

Figure 5.2 The New York Bus Cycle and a Microtrip

A Genetic Algorithm (GA) was applied to select microtrips and create a cycle that closely matched the desired cycle characteristics. In the GA, a microtrip was the gene, and a microtrip sequence was the individual. The principle of the GA and its implementation using Matlab/Simulink to the generator were introduced in reference [78, 90, 91].

\subsubsection{Database Preparation}

To generate new virtual cycles, two databases were developed: 1). microtrip database; 2). target cycle speed-time database. To produce emission data, two additional databases were prepared: 3). database of selected microtrip sequence (individual); 4). emission database from the vehicle tested.

The microtrip database for generating new virtual cycles consists of 198 microtrips from twelve target cycles. Microtrips were essentially a segment of speed-time traces. In the GA, actual microtrips were not used but identifiers were used to represent the actual microtrip. Thus a microtrip database was essentially an identifier database and a microtrip sequence was the 
identifier sequence. A microtrip identifier consisted of three numbers: cycle identifier, start time marker of the microtrip, and end time marker of the microtrip. The structure of the microtrip database was:

- 198 by 3 matrix;

- First column: cycle identifiers from 1 to 12 representing each of the twelve target cycles respectively;

- Second column: start time markers of specific microtrips in the given cycle;

- Third column: end time markers of specific microtrips in the given cycle.

The individual database consisted of microtrip sequences of new virtual cycles, which was a 368x135 matrix. The selected individual which corresponded to a new virtual cycle was stored during the cycle generation process and it was used later to extract emission data from the emissions database. To be noted, if the actual speed-time trace of a new virtual cycle was stored but without their identifiers, it would be difficult to extract corresponding emission data of the virtual cycle later.

The emission database consisted of target speed-time trace and second-by-second emission (g/s) and fuel consumption rate (gal/s) data. Due to the various time delays of emission species through the dilution tunnel, for each test run data and each emission species, timealignment with target vehicle speed was performed. Dispersion could also affect the accuracy of measurement of continuous emission data [92]. But it was not considered in this study.

Then results from the repeat tests were averaged. Emission databases varied with different vehicles tested. In this study, eight emission databases were prepared for those buses selected as backbone vehicles. If new test data become available and are ready to be employed by the IBIS, a new emission database must be prepared while the other three databases could remain the same given the same set of twelve cycles was tested.

\subsubsection{Description of Cycle Generator}

The main modules of the cycle generator included: input graphical user interface (GUI), output GUI, cycle parameter calculator and GA operators.

The input GUI allows users to input desired values of the five cycle parameters (selected from the previous chapter) associated with weight and maximum relative error for each parameter. Weight and maximum relative error were used to calculate the performance index for 
evaluation of individuals. The input GUI could define the maximum number of microtrips for each individual, number of generations and number of individuals for each population.

The cycle parameter calculator combined selected microtrips to form each individual cycle in the current generation then calculated the five parameters of the cycle. The values of the five parameters were then compared with those desired values users input. Based on the comparison, weights of parameters and maximum relative errors as input by users were used to assess the performance index.

The GA operator selected the best individual and retained it in the next generation. Based on roulette selection, mutation and crossover were performed. Mutation allowed one random microtrip of a cycle to be replaced with one random microtrip from the database or with a different microtrip from the same cycle. Crossover selected random break points in two different individuals then performed crossover after the break points between the two individuals.

The output GUI could demonstrate the newly generated virtual cycle with the values of the five cycle parameters and the non-decreasing performance history along with the generation number. If the performance history was not satisfied indicating significant errors existed between the desired and selected cycle parameters, a new cycle generation process could be resumed based on the previous result.

\subsubsection{Generation of Emissions and Fuel Economy Data}

To expand the database for backbone modeling, Figure 5.3 shows how the emissions data of the newly generated virtual cycles were acquired and how the expanded emissions database was prepared.

It was assumed that relative positions (history or sequence) of microtrips in a cycle would not significantly affect emissions and fuel consumption rates. For example, in a newly generated virtual cycle the adjacent microtrips of a microtrip could have been replaced with other microtrips. These changes were assumed not to significantly affect the emissions and fuel consumption values of the microtrip in its new virtual cycle. In this way, emissions and fuel consumption rates could be extracted as microtrip units from original standard cycles and transferred to virtual cycles as newly generated second-by-second data sequences. And these continuous data sequences were assumed to have similar values from emissions testing of the 
same vehicles on the same virtual cycles. As a result, continuous data were integrated as distance-specific values and used for modeling purposes.

The preliminary experimental validations of virtual cycles associated with emissions data are provided in Section 5.4 of this chapter.

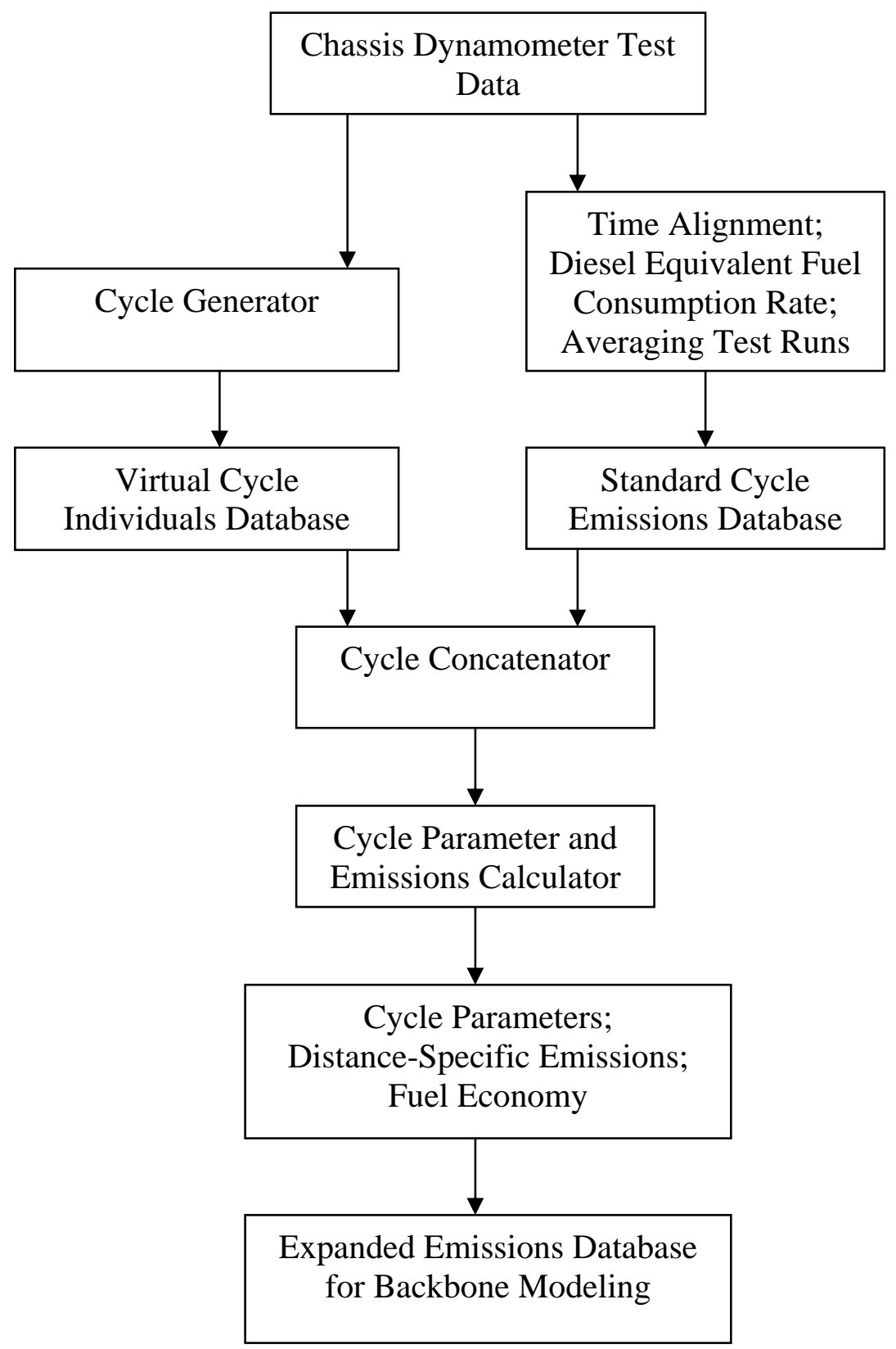

Figure 5.3 Flowchart for Expanding the Emissions Database for Backbone Modeling 


\subsection{Results and Discussions}

\subsubsection{Generation of New Virtual Cycles}

Over 360 cycles were generated to fill sparse areas in the cycle parameter planes as shown in Figure 5.1. Four cycle parameter planes were used and each plane had average speed with idle as the $X$ axis with one of other four parameters as the $Y$ axis. In total, 368 new virtual cycles were generated. These virtual cycles showed durations ranging from 100 to 4500 seconds and travel distance ranging from 0.04 to 35 miles. Some virtual cycles might contain the same micro-trips multiple times.

An example of new virtual cycles and the limits were shown in Figure 5.4 - Figure 5.7. Extensive effort to generate cycles outside those limits failed, indicating the possible operation limits of transit buses. Additionally, they showed the success of the new virtual cycles filling up sparse areas. It was noted that the original domain were significantly expanded while keeping the original trends.

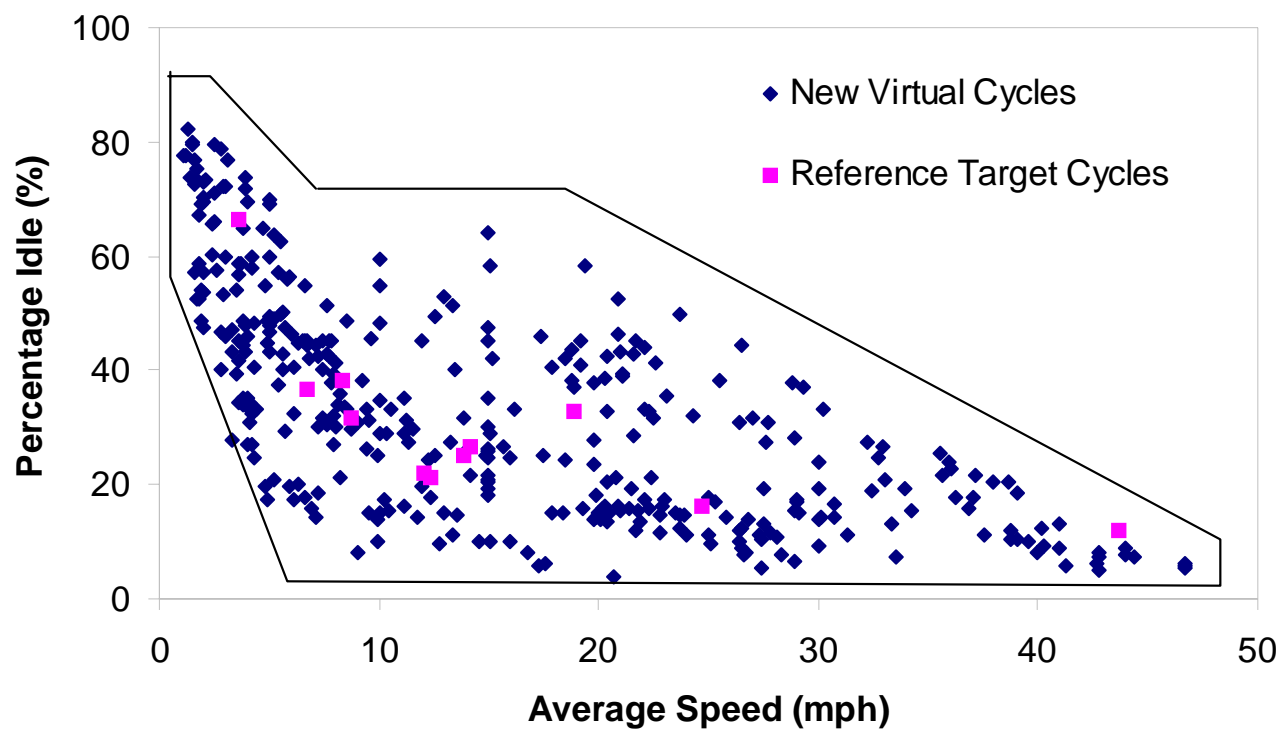

Figure 5.4 New Virtual Cycles and Limits on Percentage Idle vs. Average Speed Plane 


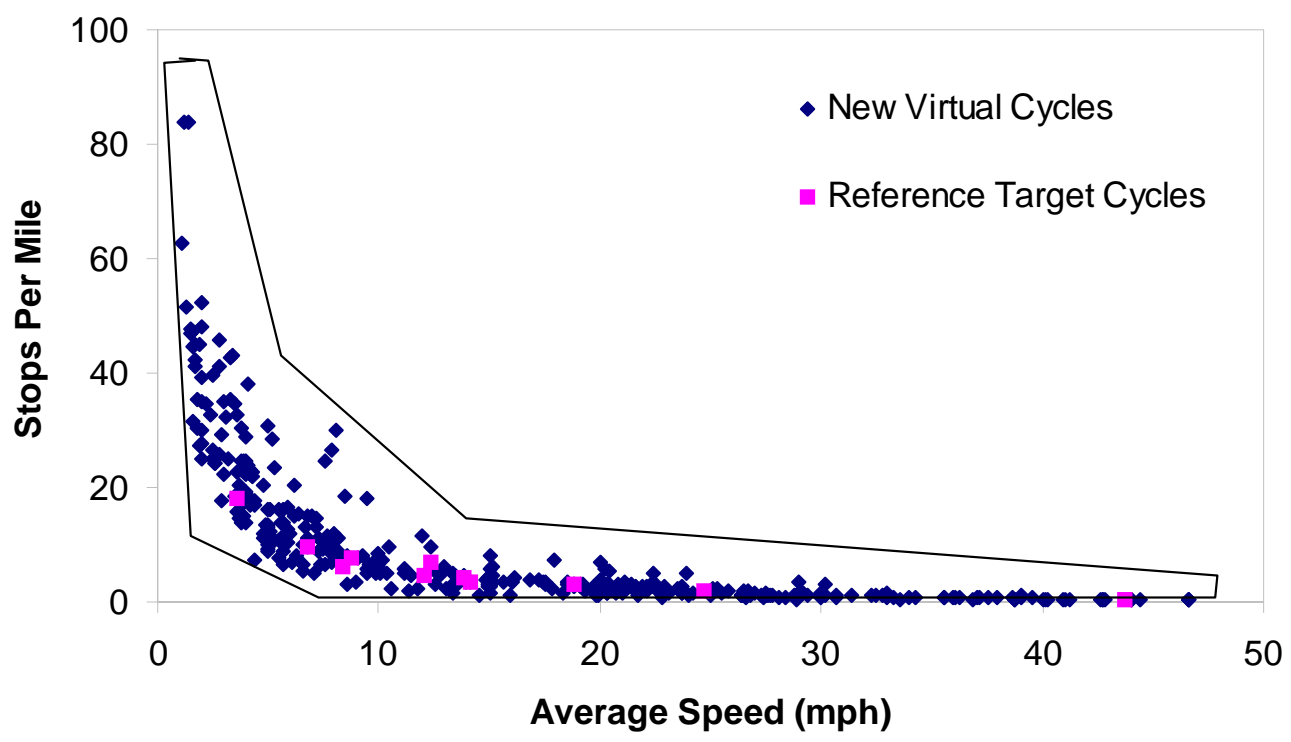

Figure 5.5 New Virtual Cycles and Limits on Stops/mile vs. Average Speed Plane

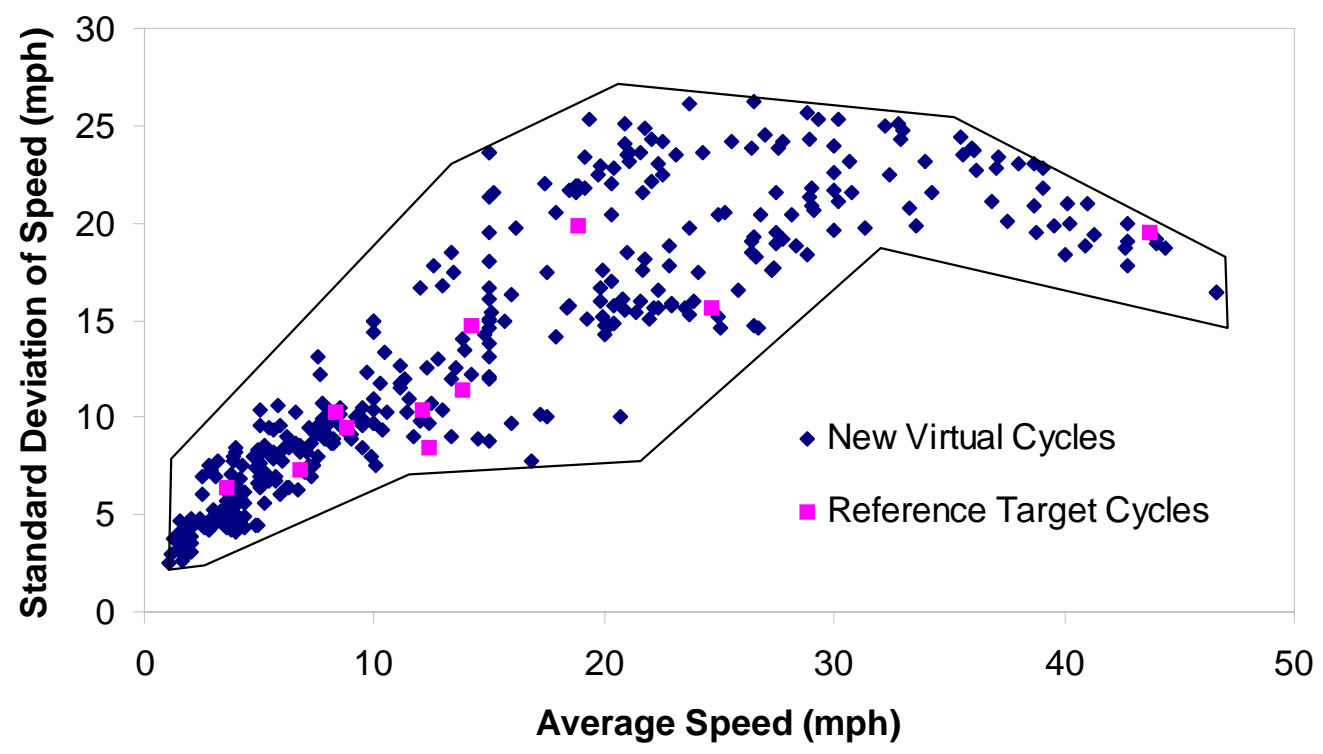

Figure 5.6 New Virtual Cycles and Limits on Standard Deviation of Vehicle Speed vs. Average Speed Plane 


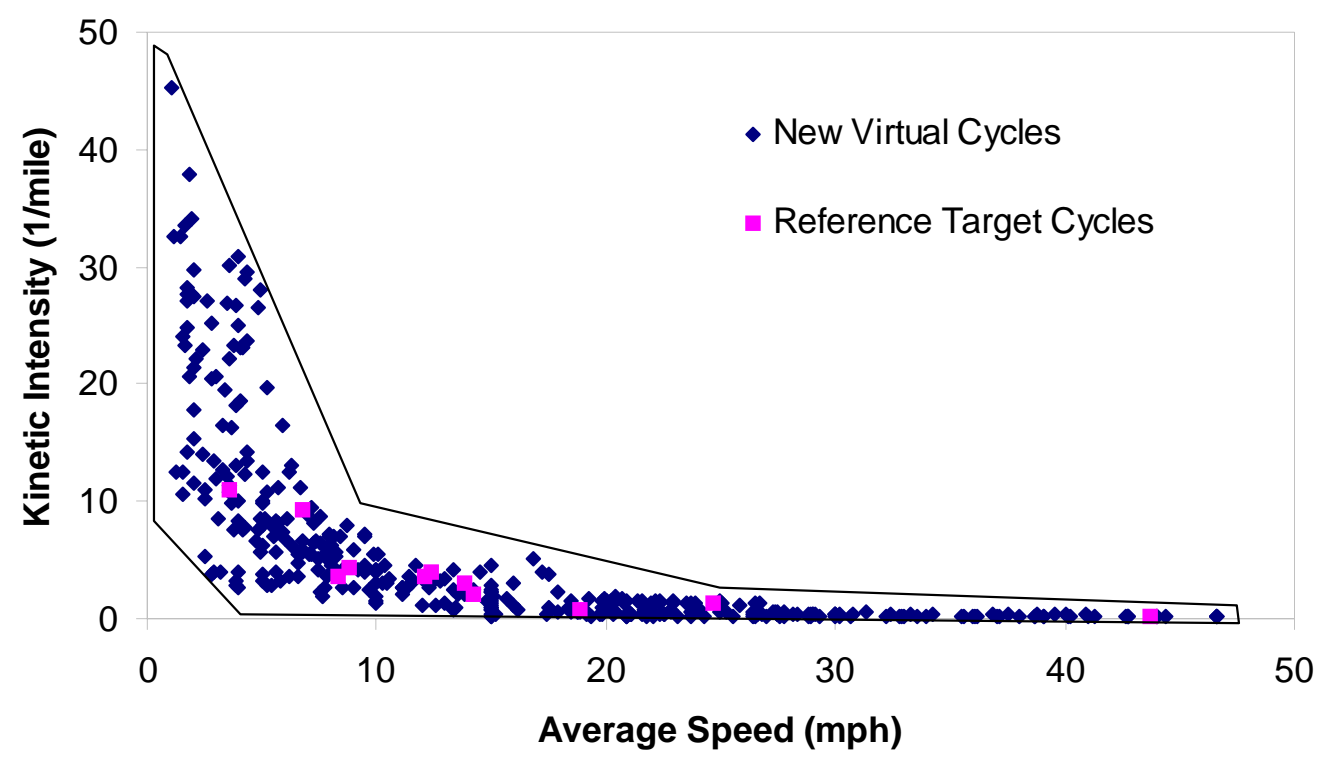

Figure 5.7 New Virtual Cycles and Limits on Kinetic Intensity vs. Average Speed Plane

\subsubsection{Generation of Emissions and Fuel Economy Data}

The same model year 2000 diesel bus as presented in Chapter 4 was used as an example here to show vast amount of emissions and fuel economy data produced with new virtual cycles. Emissions and fuel economy as a function of average speed were shown in Figure 5.8 - Figure 5.12 .

Emissions showed similar trends - decreasing with increasing average speed. Emissions had fairly strong non-linear (power) relationship with average speed with high $R$ squared values for all emissions above 0.9 except for NOx with 0.88 . Preliminary regression analysis in Chapter 4 also found power fittings were best overall for average speed based single parameter models.

Newly generated data followed the same trend as the original data and overlapped fairly well. Smaller variations of emissions were observed at average speed above $10 \mathrm{mph}$ compared to those at average speed below $10 \mathrm{mph}$. This might be because at lower average speed other cycle parameters contributed to the response variables. However, at higher average speed when driving conditions became more steady comparable to cruise mode, average speed became dominative for the response variables.

For $\mathrm{CO}_{2}$ emissions, Figure 5.8 shows it was fairly predictable even using average speed with idle (or average speed) only, which might be because $\mathrm{CO}_{2}$ emissions were highly sensitive 
to cruise features of vehicle operation that might highly correlate with average speed. However, at lower average speed, $\mathrm{CO}_{2}$ showed variation over $1000 \mathrm{~g} / \mathrm{mile}$ (up to 30\%), which implied that a second parameter should be considered for model improvement.

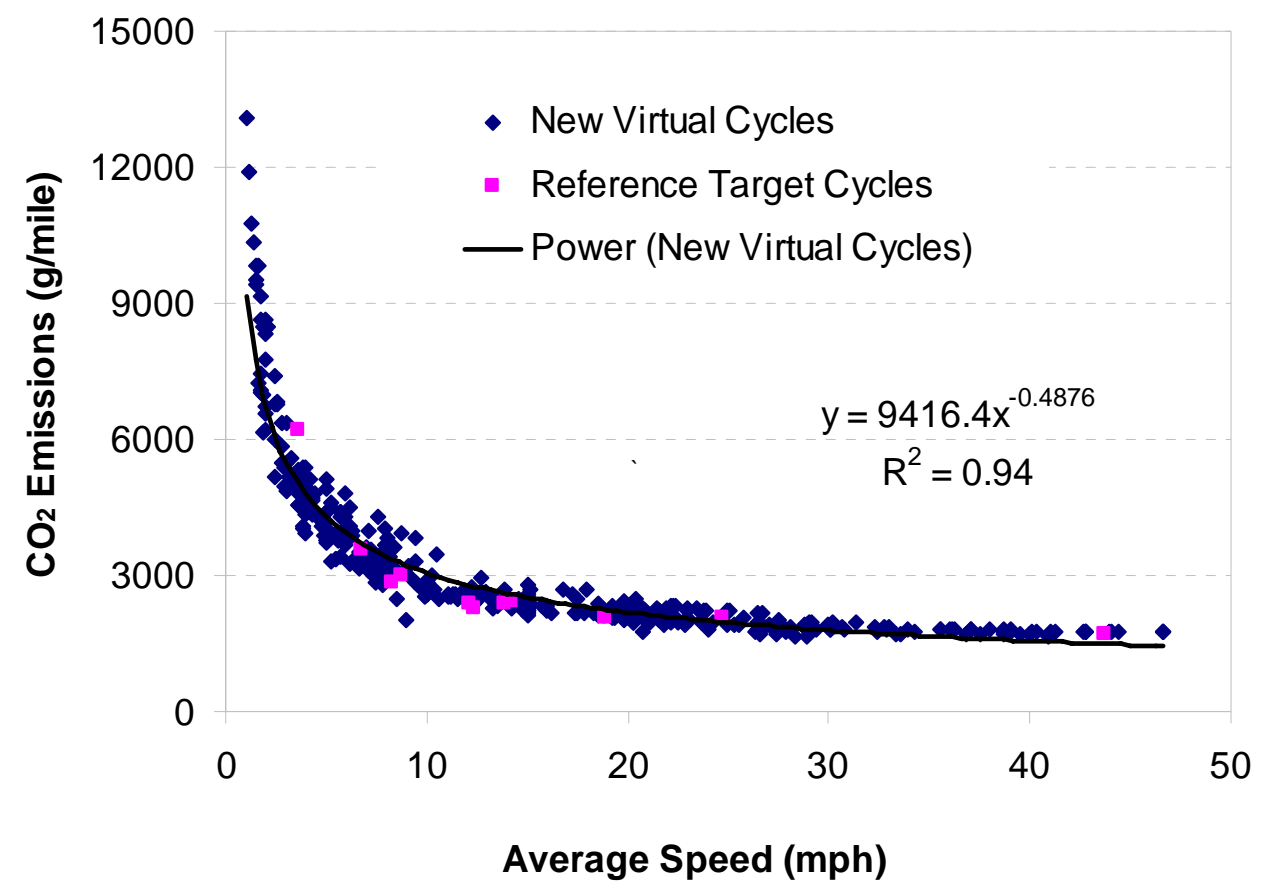

Figure $5.8 \mathrm{CO}_{2}$ Emissions vs. Average Speed

For $\mathrm{CO}$ and $\mathrm{HC}$ emissions shown in Figure 5.9 and Figure 5.10, respectively, more variations were observed at low average speed. Similar to $\mathrm{CO}_{2}$ emissions, they were quite predicable at higher average speed. HC and CO were known to be fairly sensitive to transient features of vehicle operation. Transient features could be more likely dominate at lower average speed while steady features could be more likely dominate at higher average speed. 


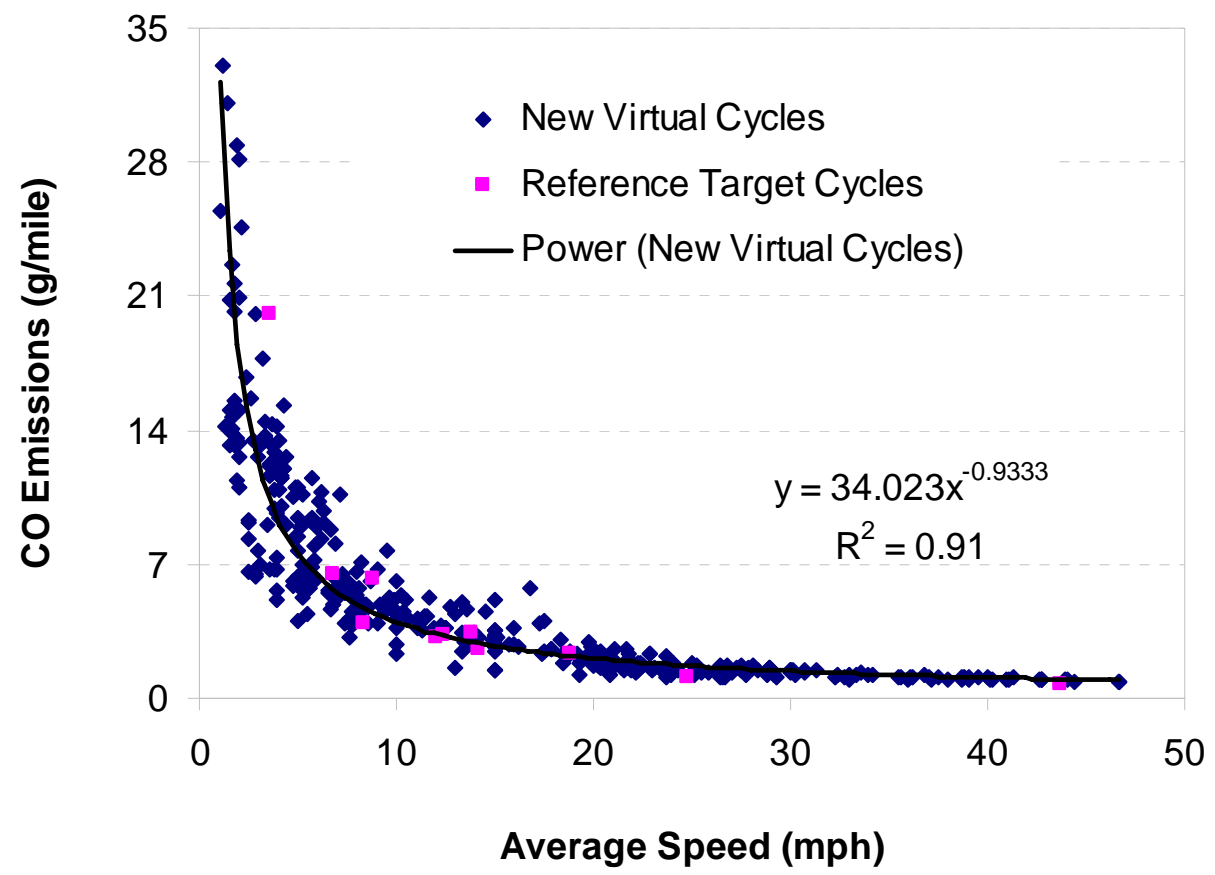

Figure 5.9 CO Emissions vs. Average Speed

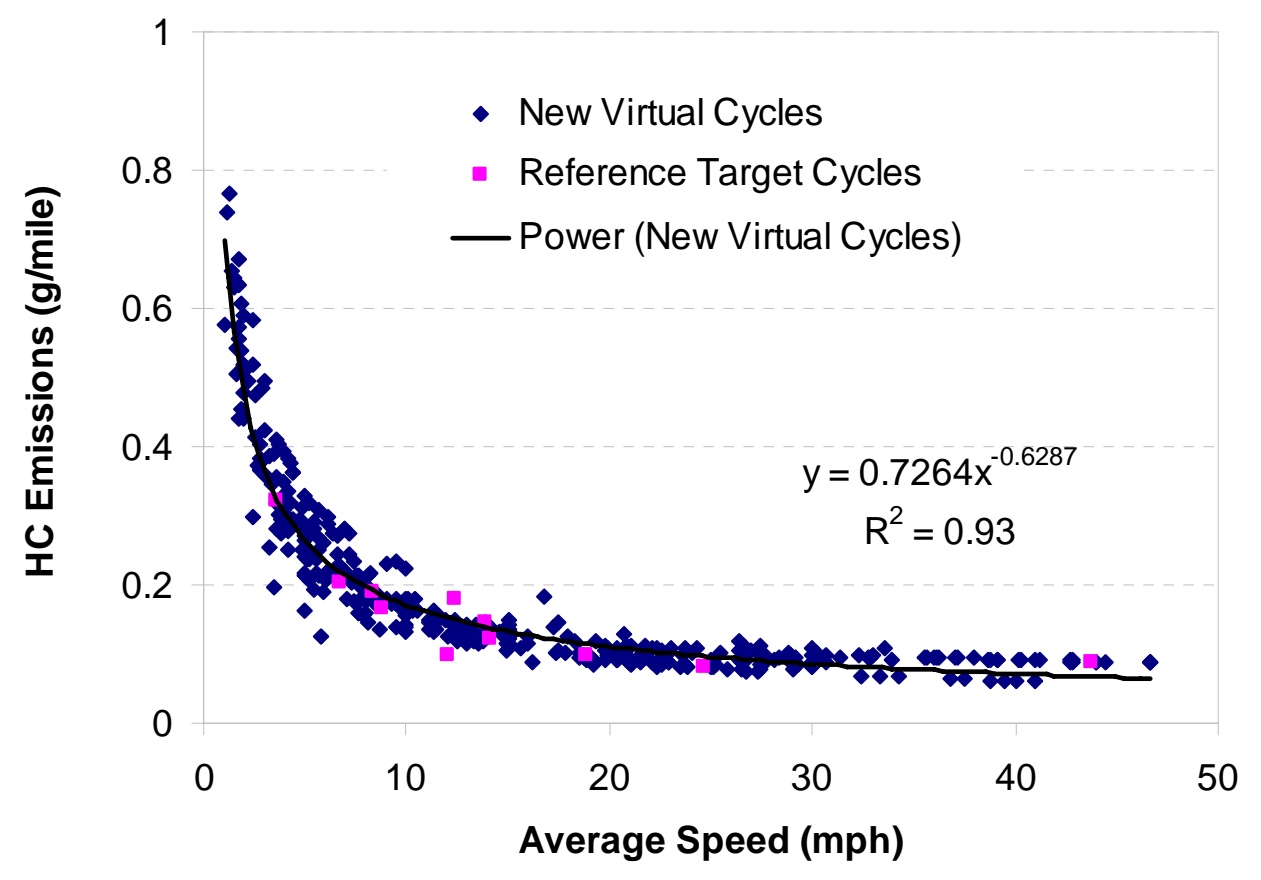

Figure 5.10 HC Emissions vs. Average Speed 
NOx emissions shown in Figure 5.11 exhibited small variations with average speed while it showed a smaller $R$ squared value of 0.88 compared with other emission species. At higher average speed, it demonstrated a flatter trend remaining nearly constant at $20 \mathrm{~g} / \mathrm{mile}$.

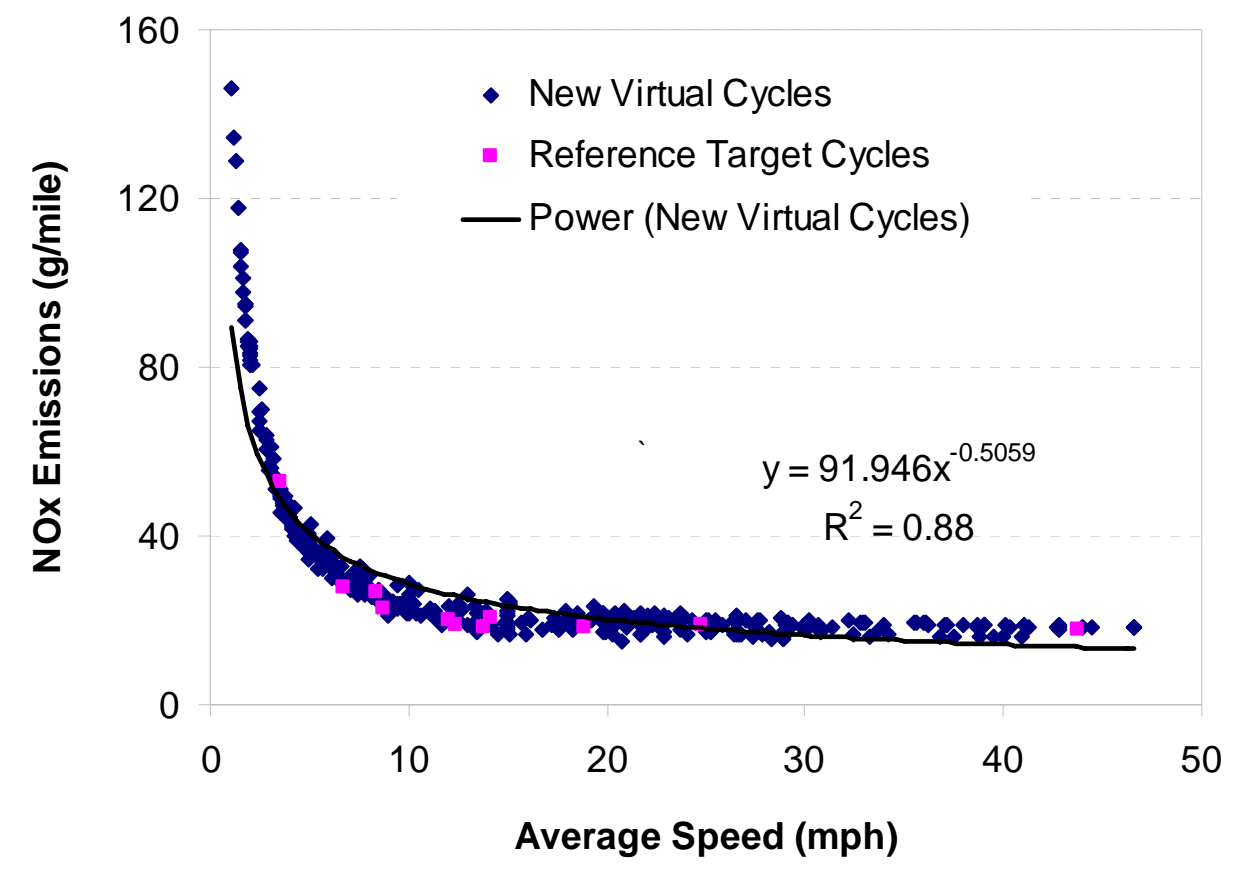

Figure 5.11 NOx Emissions vs. Average Speed

Fuel economy shown in Figure 5.12 illustrated that it increased with increased average speed and showed fairly strong logarithm relationship with average speed at $R$ squared of 0.95. However, up to $1.5 \mathrm{mpg}$ variations of fuel economy were observed at average speed above 10 mph, which were estimated at $10 \%$ - $40 \%$ variation along the average speed. 


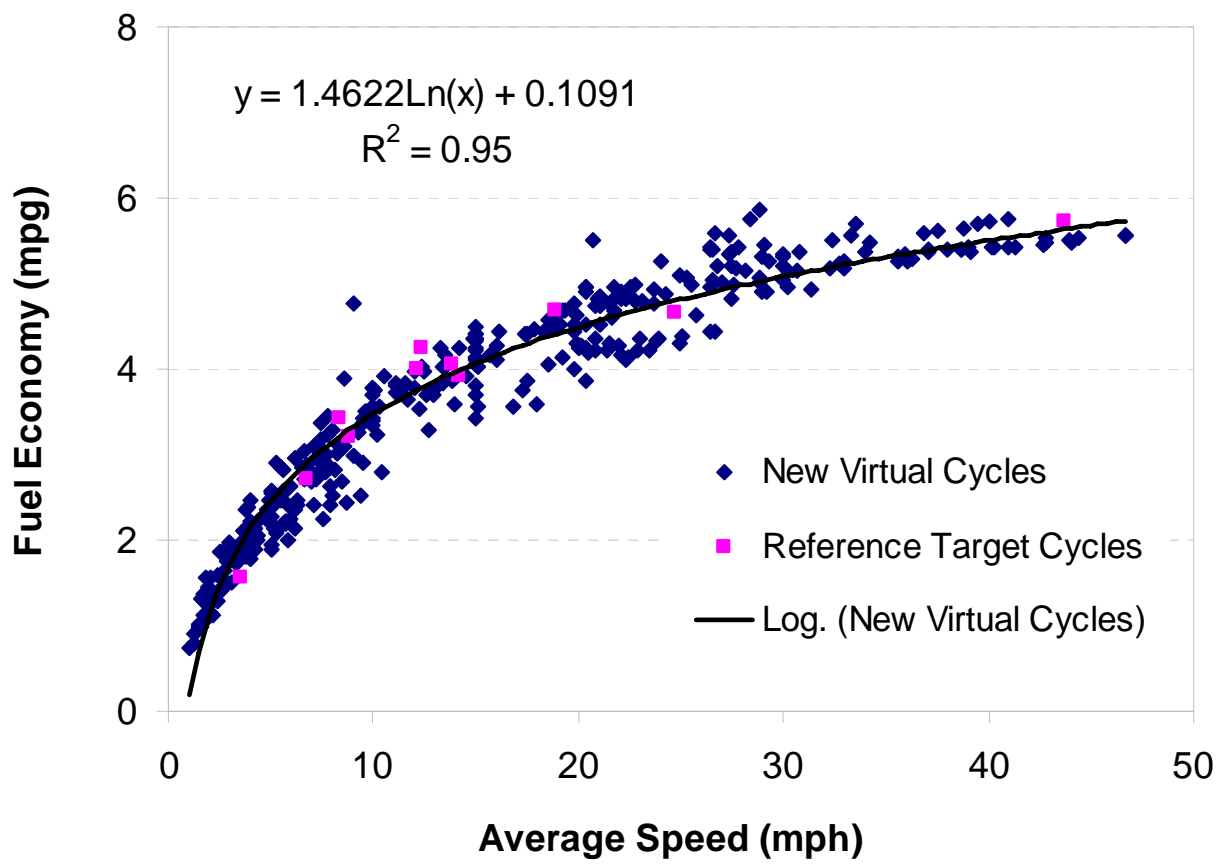

Figure 5.12 Fuel Economy vs. Average Speed

These figures demonstrate that duty cycle and emissions generation was effective in filling sparse areas in both cycle metric and emissions planes. In addition, it confirmed the dominant role of average speed as an independent variable in fitting curves. This provided insight into how to select polynomial terms during the development of backbone models based on two cycle parameters which is discussed in the next chapter.

However, some variations were observed in a certain range of average speed for emissions and fuel economy, reinforcing that average speed alone might not accurately estimate the response variables.

\subsection{Preliminary Experimental Validation of Cycle and Emissions Generation}

Three virtual cycles (VC) were selected for emissions testing to validate the cycle generation method. Based on the test results, the validation served three purposes as follows:

1) To determine if the vehicle could follow the target speed-time traces to complete the emissions test on a chassis dynamometer. If it was difficult for a driver or vehicle to complete or closely follow the target speed trace, it might indicate that the virtual cycle was not realistic.

2) In order to generate virtual cycles from existing microtrips, the generation method selected microtrips and their associated emissions and fuel consumption rates data from the 
target cycle speed-time and emissions database and then concatenated them into a new virtual cycle. As a result, adjacent microtrips of some or all of the microtrips in new virtual cycles were changed compared to those in standard cycles. To investigate the impact of microtrip history (sequence or relative position) on emissions and fuel economy, the continuous emissions and fuel consumption rates were compared between the cycle generation method and emissions testing results of the virtual cycles from the same vehicle. Ideally, the cycle generation could generate the same rates as the emissions testing. If valid repeat tests were available, repeatability analysis was conducted to further evaluate the method.

3) For the development of the IBIS emissions and fuel economy models, integrated data from the cycle generation were used to expand the database. In this study, integrated emissions (g/mile) and fuel economy (mpg) determined by the cycle generation method were compared with actual test results.

Figure 5.15 - Figure 5.15 provide three selected virtual cycles for emissions testing and Table 5.1 summarizes their cycle parameters. These three cycles were identified as VC_274, VC_637 and VC_28 and had average speeds of $21.63 \mathrm{mph}, 27.8 \mathrm{mph}$ and $7.42 \mathrm{mph}$, respectively. VC_274 and VC_637 had the same top speed of $58 \mathrm{mph}$ due to the same high-speed microtrip acquired from the COMM cycle. Twenty seconds of idle period were added at the beginning of each cycle. In this study, the idle period at the beginning of the cycle was excluded to make sure that virtual cycles were completely composed of microtrips from standard reference cycles. 


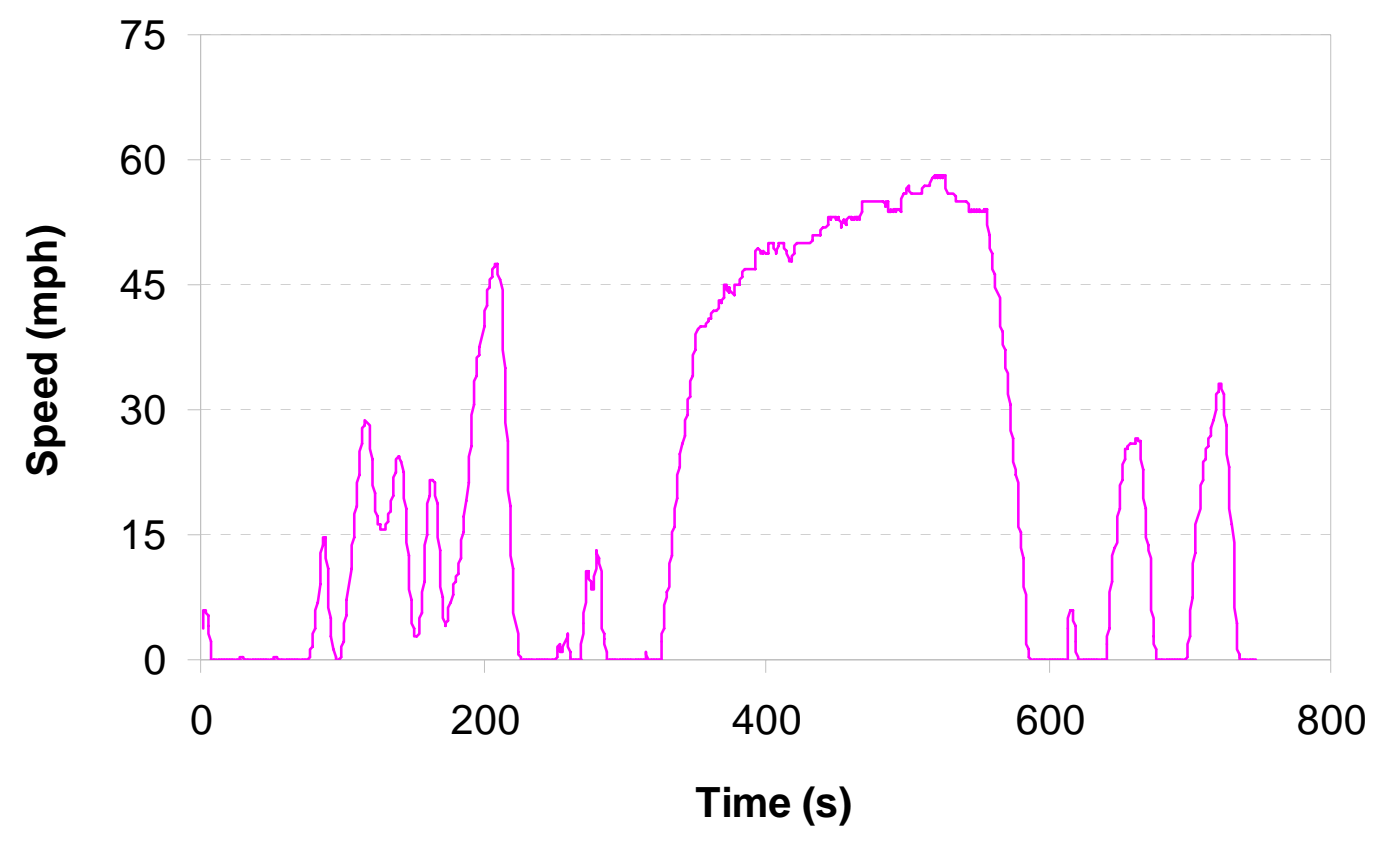

Figure 5.13 Target Speed-Time Traces of VC_274

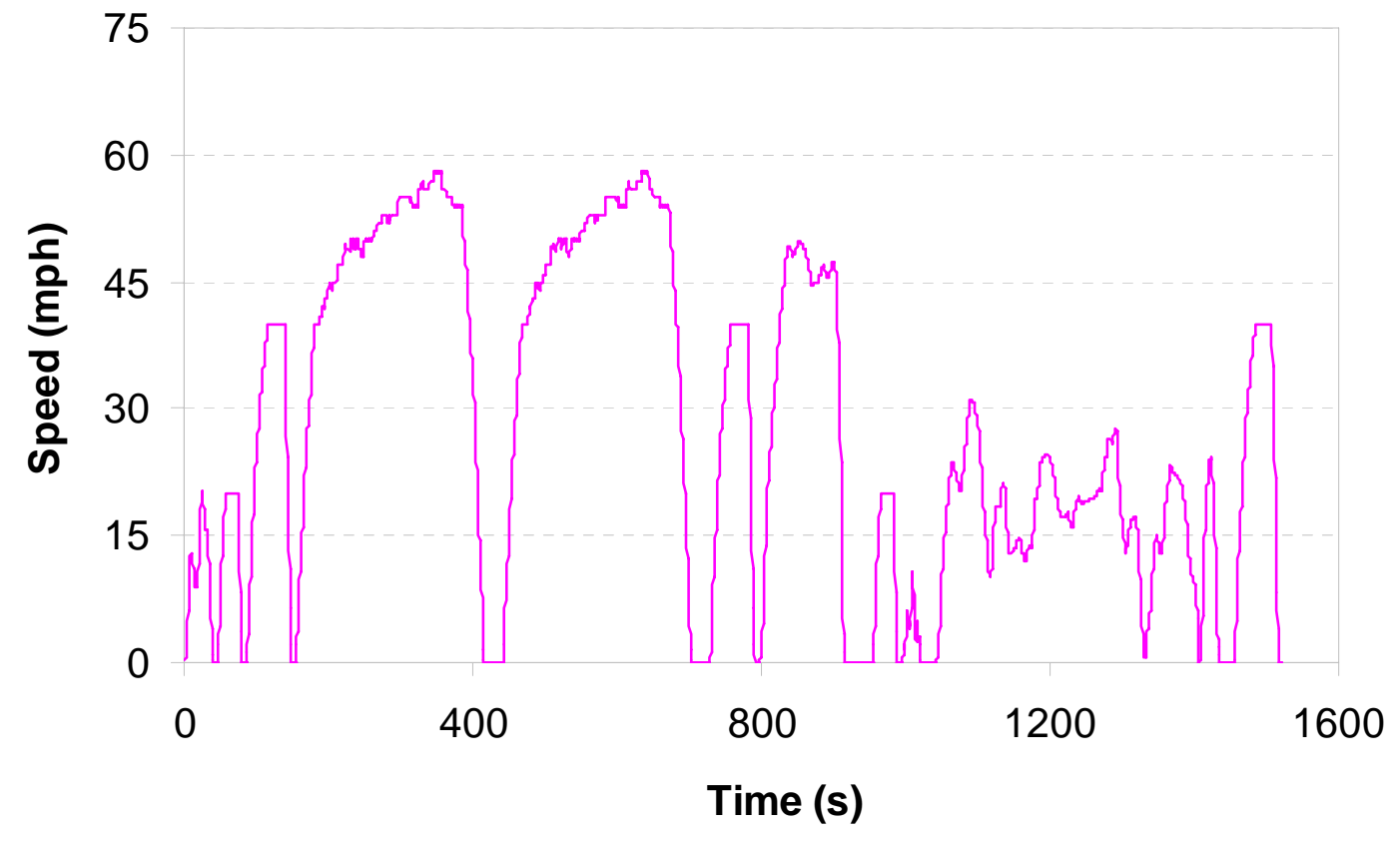

Figure 5.14 Target Speed-Time Traces of VC_637 


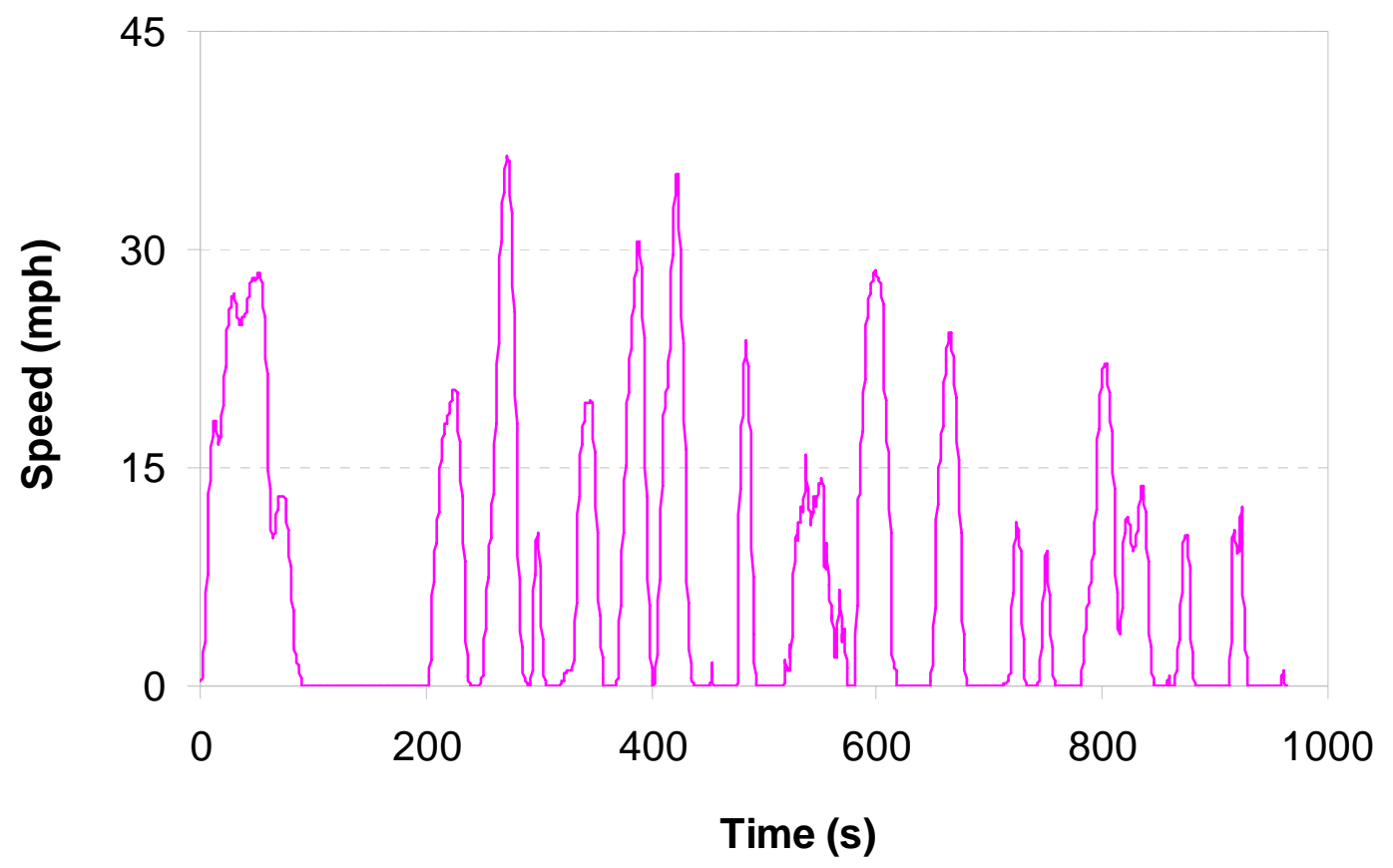

Figure 5.15 Target Speed-Time Traces of VC_28

Table 5.1 Cycle Statistics of the Selected Virtual Cycles

\begin{tabular}{|c|c|c|c|c|c|c|c|c|c|c|c|c|c|c|c|}
\hline $\begin{array}{l}0 \\
\frac{1}{0} \\
\frac{1}{0} \\
z \\
0\end{array}$ & 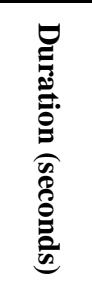 & 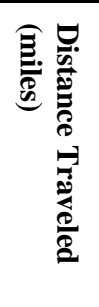 & 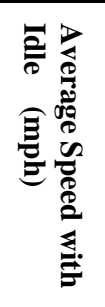 & 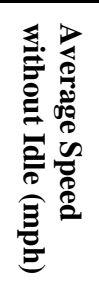 & 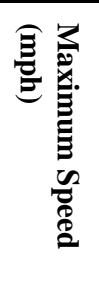 & 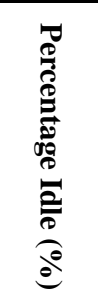 & 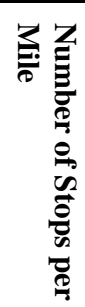 & 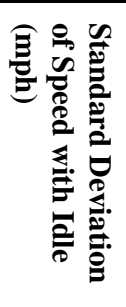 & 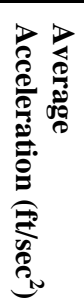 & 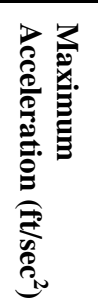 & 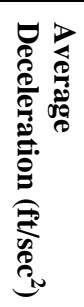 & 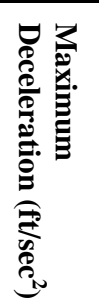 & 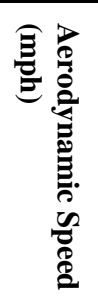 & 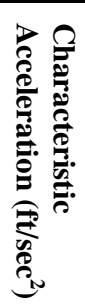 & 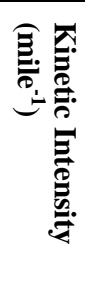 \\
\hline VC_274 & 748 & & 21.63 & 30.36 & 58 & 8.74 & 2.67 & 21.60 & 1.50 & 6.67 & 1.94 & 6.53 & 45.49 & 0.51 & 0.60 \\
\hline VC_637 & 1523 & 11.76 & 27.80 & 31.43 & 58 & 11.56 & 1.02 & 19.16 & 1.22 & 4.99 & 1.60 & 8.58 & 43.40 & 0.40 & 0.52 \\
\hline VC_28 & 965 & 1.99 & 7.42 & 12.41 & 36.5 & 40.21 & 9.55 & 9.47 & 1.64 & 6.97 & 1.98 & 6.67 & 21.17 & 0.94 & 5.12 \\
\hline
\end{tabular}

Two buses were tested over 16 standard reference cycles as well as the three virtual cycles at the WMATA test site using the WVU TransLab. The cycle generation was based on the microtrips extracted from 12 out of 16 standard reference cycles. In this way, emissions and fuel economy data were generated using the flowchart shown in Figure 5.3 for the selected virtual cycles and then the results were compared with their emissions testing results. The two buses were a MY 2008 60-ft stochiometric CNG bus equipped with the three-way catalyst (TWC) and a MY 2008 40-ft diesel hybrid-electric bus equipped with an active diesel particulate filter (DPF) and diesel oxidation catalyst (DOC). 
These two buses were also selected for backbone modeling (Chapter 6) for MY group VI and their specifications were provided in Table 6.1. The CNG bus was equipped with a Cummins ISL G 320 engine. The ISL G engine used cooled exhaust gas recirculation (EGR) and could meet EPA 2010 emissions standards [93]. The cooled EGR and the stochiometric combustion enabled ISL G engines to effectively employ TWC aftertreatment without required maintenance. The fuel economy values from the CNG bus had been converted to diesel energy equivalent fuel economy based on fuel properties.

\subsubsection{CNG Bus}

Table 5.2 provides the integrated results of emissions testing and cycle generation of the selected virtual cycles for the MY 2008 CNG bus. Two test runs were conducted over the VC_637 cycle to check its repeatability. Among the three virtual cycles, the VC_28 cycle had the lowest average speed, the highest percentage idle, the greatest number of stops per mile and the highest kinetic intensity, the lowest fuel economy and the highest distance-specific emissions with the exception of NOx and NMHC emissions. This further demonstrated that cycle characteristics affect emissions and fuel economy. In terms of the values of the above cycle metrics, emissions and fuel economy, the VC_28 cycle was followed by the VC_274 then by the VC_637 cycle (averaged values of the two repeating runs).

Table 5.2 Results of Emissions Testing and Cycle Generation of Selected Virtual Cycles from a MY 2008 CNG Bus

\begin{tabular}{|c|c|c|c|c|c|c|}
\hline \multicolumn{7}{|c|}{ Emissions Testing } \\
\hline & CO (g/mile) & $\mathrm{CO}_{2}$ (g/mile) & NOx (g/mile) & HC (g/mile) & Fuel Economy (mpg) & NMHC (g/mile) \\
\hline VC_274 & 117.85 & 2234.13 & 1.67 & 13.99 & 2.81 & -0.06 \\
\hline VC_637_1 & 84.56 & 1897.96 & 1.76 & 8.15 & 3.37 & -0.04 \\
\hline VC_637_2 & 97.31 & 1855.33 & 1.45 & 8.95 & 3.40 & -0.07 \\
\hline VC_28 & 135.35 & 4154.27 & 0.50 & 19.62 & 1.56 & -0.20 \\
\hline \multicolumn{7}{|c|}{ Cycle Generation } \\
\hline & CO (g/mile) & $\mathrm{CO}_{2}$ (g/mile) & NOx (g/mile) & HC (g/mile) & Fuel Economy (mpg) & NMHC (g/mile) \\
\hline VC_274 & 111.22 & 2212.30 & 1.80 & 13.91 & 2.85 & -0.15 \\
\hline VC_637 & 97.48 & 1872.30 & 1.33 & 10.26 & 3.36 & -0.13 \\
\hline VC_28 & 165.21 & 4091.30 & 0.78 & 26.01 & 1.56 & 0.07 \\
\hline
\end{tabular}

To be noted, the NMHC emissions were below the detection level of the instrumentation and their integrated emission results from the 12 standard reference cycles as well as the three virtual cycles were all below zero. In general, the majority of total HC emissions from the CNG 
bus were methane (over 95\%). Negative values indicated that the CNG bus exhibited nearly zero NMHC emissions. Regarding cycle generation, NMHC emissions showed negative values as well except for the VC_28 cycle.

Figure 5.16 shows the relative difference in results (except for NMHC due to negative values) between emissions testing and cycle generation. The relative difference in this section was calculated as the absolute value of the difference between emissions testing and cycle generation divided by the result of emissions testing.

It shows that the differences for $\mathrm{CO}_{2}$ and fuel economy are within $2 \%$ and $1 \%$ respectively, indicating that the cycle generation method could be relatively accurately substitute to emissions testing for them. However, the difference for CO ranges from $6 \%$ to $22 \%$, NOx from $8 \%$ to $57 \%$ and $\mathrm{HC}$ from $1 \%$ to $33 \%$. The large differences associated with their wide variation might indicate that reliable $\mathrm{CO}, \mathrm{NOx}$ and $\mathrm{HC}$ emission values were more difficult to acquire using the cycle generation method compared to $\mathrm{CO}_{2}$ and fuel economy data. Due to the high sensitivity to transient features of driving conditions, $\mathrm{CO}$ and $\mathrm{HC}$ emissions could be significantly different even from repeating test runs, which is further discussed later this section. In addition, those differences might be attributed to the impact of microtrip history as well as the dispersion.

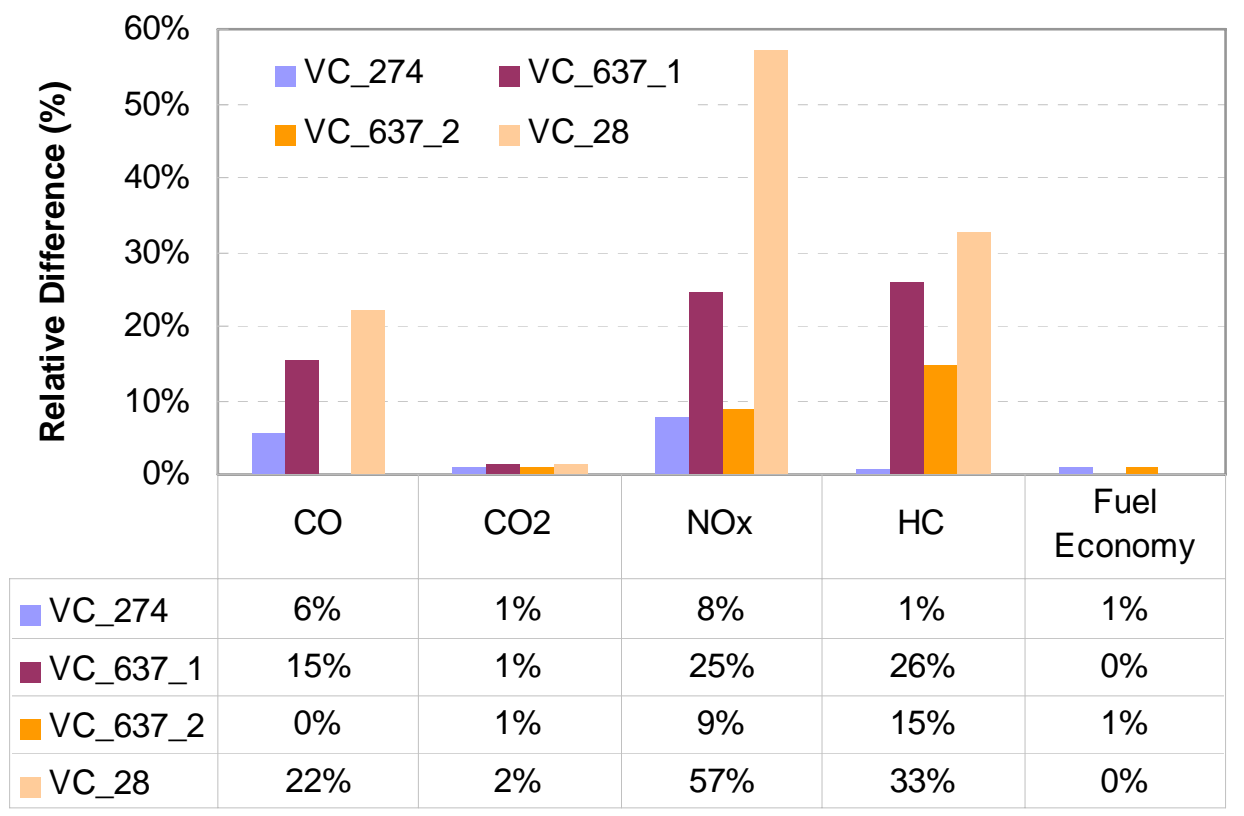

Figure 5.16 Relative Difference between Emissions Testing and Cycle Generation of Selected Virtual Cycles from a MY 2008 CNG Bus 
It was noted that $\mathrm{CO}, \mathrm{HC}$ and NOx emissions showed the same pattern in terms of relative difference shown in Figure 5.16 across the three virtual cycles - the VC_274 cycle had the smallest difference while the VC_28 cycle showed the largest difference. As described above, the VC_28 cycle presented the extreme values to the other two cycles. As a result, it showed significantly higher difference compared to other two cycles.

Figure 5.17 provides the actual and target speed-time traces as well as the second-bysecond fuel consumption and $\mathrm{CO}_{2}$ emission rates from emissions testing and cycle generation over the VC_637 cycle while Figure 5.18 shows HC, CO and NOx emission rates. Figures B-1 to B-4 in Appendix B show the results for VC_274 and VC_28 cycles. At the right side of these figures, parity plots were provided. Each of these plots show two repeating test runs of the VC_637 cycle. The comparisons of speed-time traces were intended to show whether the virtual cycles were realistic so that the driver could finish and closely follow target speed-time traces. The comparisons of continuous fuel consumption and emissions could demonstrate how close the cycle generation values were to the experimental values and consequently provide the insight on whether the microtrip history could affect fuel consumption and emissions rates.

The values of the $R$ squared and slope of the linear regression lines from the parity plots could be used to evaluate the precision and accuracy respectively [94] between experimental and cycle generation results. Ideally, if results were the same both $R$ squared and slope values would be one which corresponded to the trend line of $y=x$. The greater deviation from the $y=x$ line indicates lower accuracy or precision. 

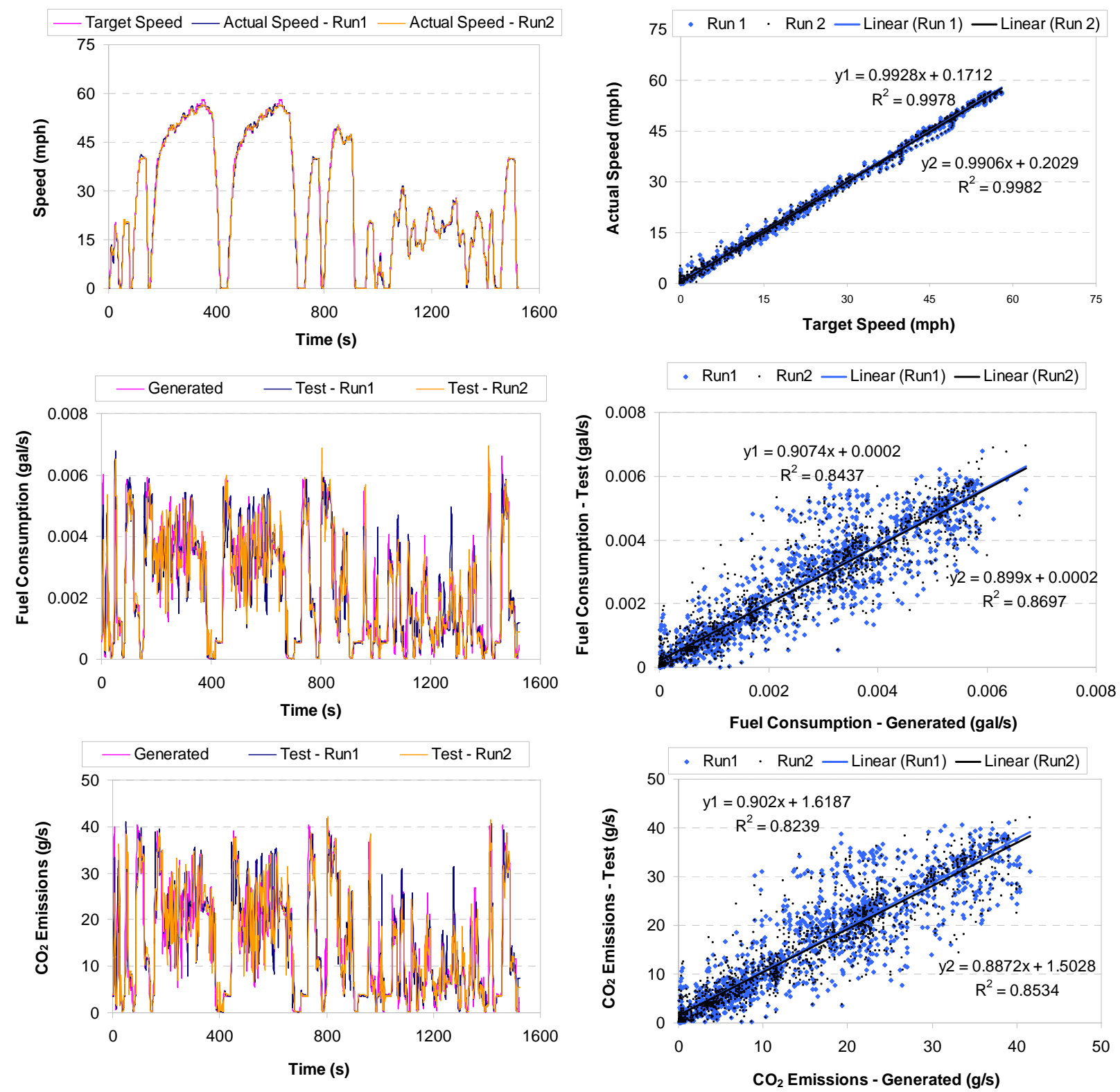

Figure 5.17 Target and Actual Vehicle Speed, Fuel Consumption and $\mathrm{CO}_{2}$ Rates of Emissions Testing and Cycle Generation of VC_637 from a MY 2008 CNG Bus

Figure 5.17 shows that the driver could accurately and precisely follow the target speedtime traces for both of test runs with $R$ squared and slope values all close to one (within 1\%). Regarding fuel consumption and $\mathrm{CO}_{2}$ rates, it shows that the cycle generation method could accurately represent the emissions test results with slope values above 0.88 . Some data scattered and resulted in lower $R$ squared values but still all values were 0.82 or above, indicating moderate precision of results for the cycle generation method compared to the emissions testing. 
The regression results from the parity plots of three virtual cycles are shown in Table 5.3. Regarding the other two virtual cycles, similarly, results showed that the driver could accurately and precisely follow the target speed-time traces with $R$ squared and slope values all above 0.95. The fuel consumption and $\mathrm{CO}_{2}$ rates from the cycle generation could accurately and moderately precisely represent the emissions testing with slope values of 0.92 or above and $R$ squared of 0.86 or above.

Table 5.3 Regression Results from Parity Plots of Selected Virtual Cycles from a MY 2008 CNG Bus

\begin{tabular}{lllllllc}
\hline & & Speed & CO & CO $_{2}$ & NOx & HC & Fuel Consumption \\
\hline \multirow{2}{*}{ VC_274 } & Slope & 0.98 & 0.62 & 0.92 & 0.76 & 0.48 & 0.93 \\
& $\mathrm{R}^{2}$ & $>0.99$ & 0.39 & 0.87 & 0.71 & 0.27 & 0.88 \\
\hline \multirow{2}{*}{ VC_637_1 } & Slope & $>0.99$ & 0.40 & 0.92 & 0.79 & 0.32 & 0.91 \\
& $\mathrm{R}^{2}$ & $>0.99$ & 0.27 & 0.82 & 0.60 & 0.23 & 0.84 \\
\hline \multirow{2}{*}{ VC_637_2 } & Slope & $>0.99$ & 0.54 & 0.89 & 0.69 & 0.41 & 0.90 \\
& $\mathrm{R}^{2}$ & $>0.99$ & 0.32 & 0.85 & 0.58 & 0.24 & 0.87 \\
\hline \multirow{2}{*}{ VC_28 } & Slope & 0.97 & 0.56 & 0.99 & 0.22 & 0.39 & 0.96 \\
& $\mathrm{R}^{2}$ & $>0.99$ & 0.38 & 0.86 & 0.10 & 0.23 & 0.86 \\
\hline
\end{tabular}

As previously described, the relative positions or history of some or all microtrips in the virtual cycles had been changed compared to these of standard cycles. Based on the above analysis, it might indicate that fuel consumption and $\mathrm{CO}_{2}$ emission rates for the $\mathrm{CNG}$ bus were not sensitive to the microtrip history so that different order of the same set of microtrips could lead to very similar results as long as other factors from emissions testing were consistent.

Figure 5.18 and Figure B-1 to Figure B-4 show that HC, CO and NOx emissions present more scattered points in the parity plots than $\mathrm{CO}_{2}$ and fuel consumption. Their slopes and $R$ squared values were much lower than those of the latter while NOx emissions had better values than HC and CO emissions except those from the VC_28 cycles. Compared to the other two cycles, the VC_28 cycle had significantly lower average speed and much higher values in terms of percentage idle, and number of stops per mile. It might indicate that the cycle generation method could fairly represent the NOx emission values from tests at higher average speed while the method could approximately represented CO and HC emissions values from tests. Regarding $\mathrm{CO}$ and $\mathrm{HC}$ emissions, their larger differences between emissions testing and cycle generation might be partially attributed to the changed mictrotrip history from the cycle generation. They 
were sensitive to the transient features of driving conditions and a minor change from cycle characteristics might lead to significant difference in emissions.
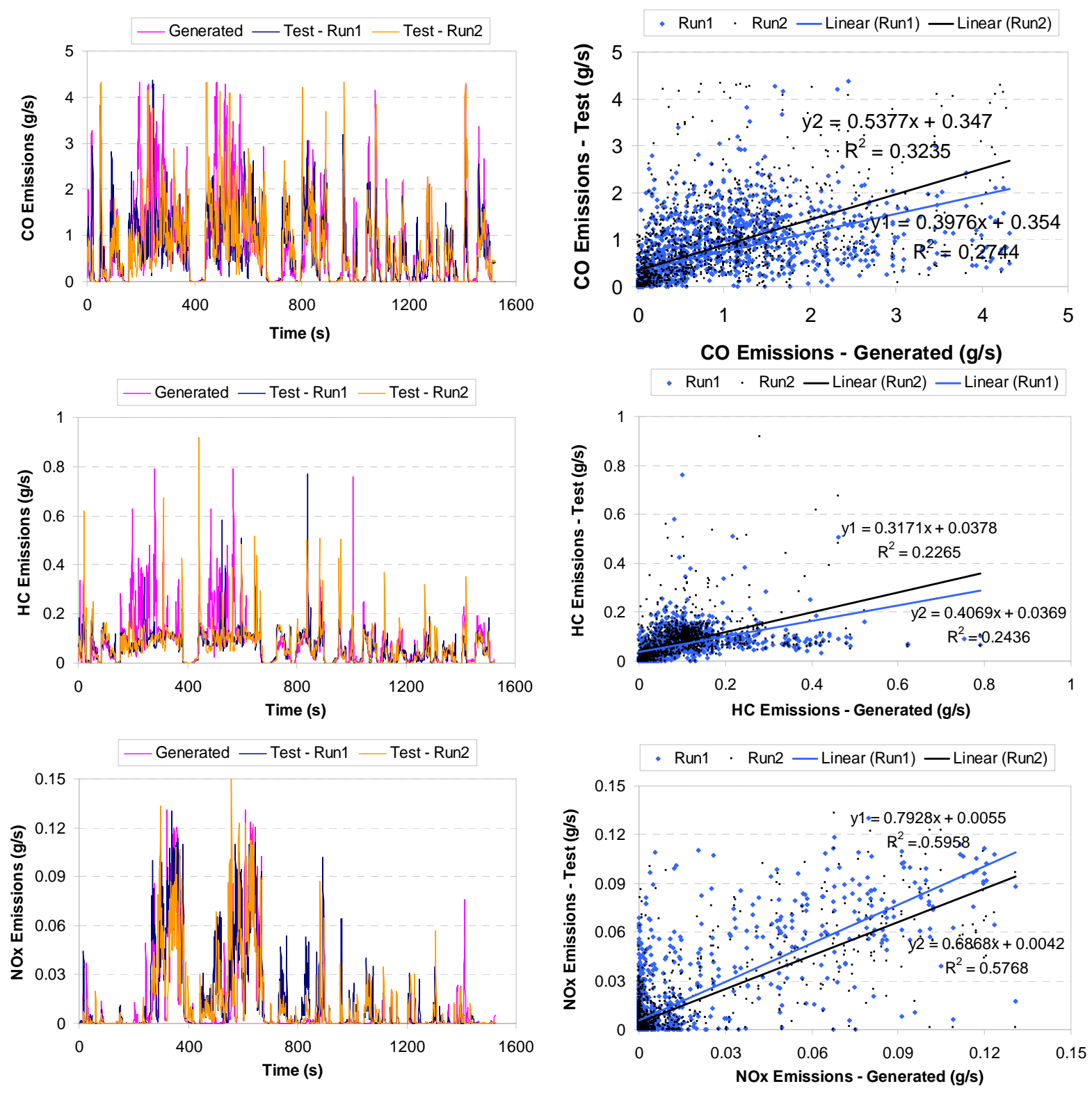

Figure 5.18 CO, HC and NOx Rates from Emissions Testing and Cycle Generation of VC_637 from a MY 2008 CNG Bus

In addition, it was noted that the near zero NOx emission rates from this CNG bus might be primarily attributed to the stoichiometric engine equipped with the cooled EGR and TWC aftertreatment. A previous US study [95] and European studies [96, 97] also suggested that schoichiometric engine with cooled EGR and TWC could significantly reduce NOx emissions when compared to lean burn CNG engines. Microtrip history could affect thermal history and the 
efficiency of the aftertreatment devices. For this CNG bus, different microtrip history from the cycle generation might lead to different levels for HC, CO and NOx emissions, which could be an additional contributor to scattering points shown in Figure 5.18.

Figure 5.19 illustrates the repeatability of the VC_637 cycle with comparisons between two repeat test runs in terms of vehicle speed, fuel consumption and emission rates.
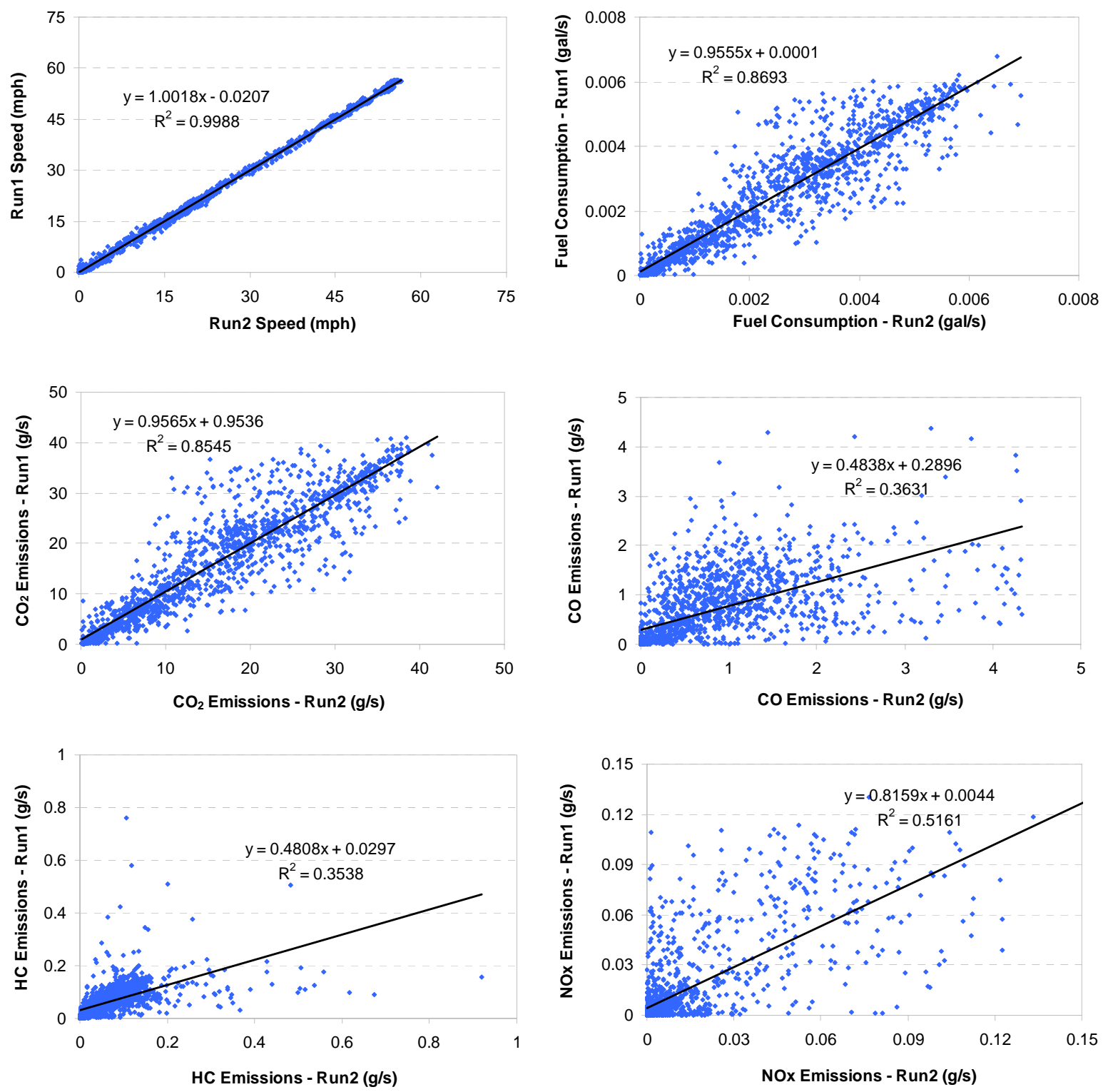

Figure 5.19 Repeatability of Vehicle Speed, Fuel Consumption and Emission Rates over the VC_637 Cycle from a MY 2008 CNG Bus

The major difference of Figure 5.19 compared to Figure 5.17 and Figure 5.18 was that there was no change in microtrip history in Figure 5.19 due to repeat test runs. The repeat tests 
could provide further insight into the impacts of microtrip history on emissions and fuel consumption. If significant differences existed between comparison of results from repeat tests (Figure 5.19) and those of emissions testing and cycle generation (Figure 5.17 and Figure 5.18), it might indicate that microtrip history could be one of the primary contributors to the difference between emissions testing and cycle generation.

Given the values of the slopes and $R$ squared, the results showed relatively high repeatability for vehicle speed as well as for fuel consumption and $\mathrm{CO}_{2}$ emission rates. Similarly, it showed more scattering of data points for $\mathrm{CO}, \mathrm{HC}$ and NOx emissions while NOx presented higher accuracy and precision than those of $\mathrm{CO}$ and HC. However, Figure 5.19 does not demonstrate significant improvement when compared to Figure 5.17 and Figure 5.18 with respect to values of slopes and $R$ squared from the parity plots. Test conditions of repeat runs were intended to remain consistent but results still showed significant variability from test runs for $\mathrm{HC}$ and $\mathrm{CO}$. It indicates that different results between emissions testing and cycle generation for HC and CO might be not only due to the changed microtrip history but also because of their high sensitivity to transient features of driving conditions.

Figure 5.20 shows the repeatability for vehicle acceleration. Compared to vehicle speed, vehicle acceleration presented lower repeatability given the smaller values of the slope and $R$ squared. The small variability from acceleration might be one of the major contributors to the high variability of $\mathrm{CO}$ and $\mathrm{HC}$ emissions.
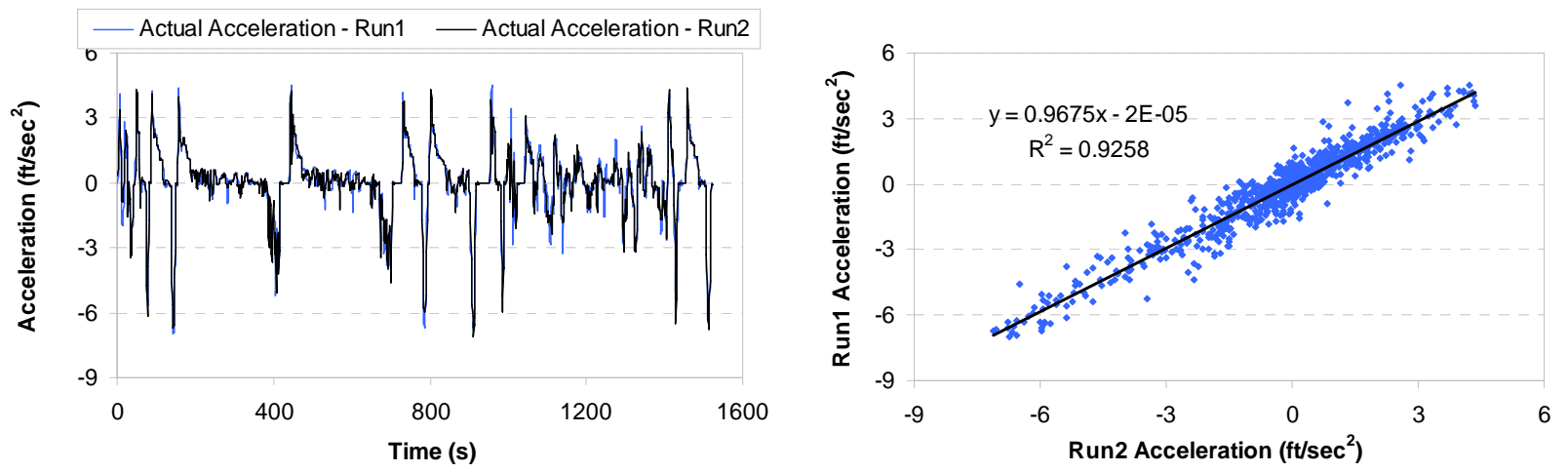

Figure 5.20 Repeatability of Vehicle Acceleration on the VC_637 Cycle from a MY 2008 CNG Bus

Figure 5.21 illustrates the NMHC emissions rates from the CNG bus. It shows that most NMHC rates are near zero except for some spikes. As described previously, the integrated values of NMHC were negative and this CNG bus had near zero emission level for NMHC. The parity 
plot shows similar trends for the two test runs with low values of slopes and $R$ squared which might be primarily due to those spikes that did not occur at the exactly same time during emissions testing and cycle generation.
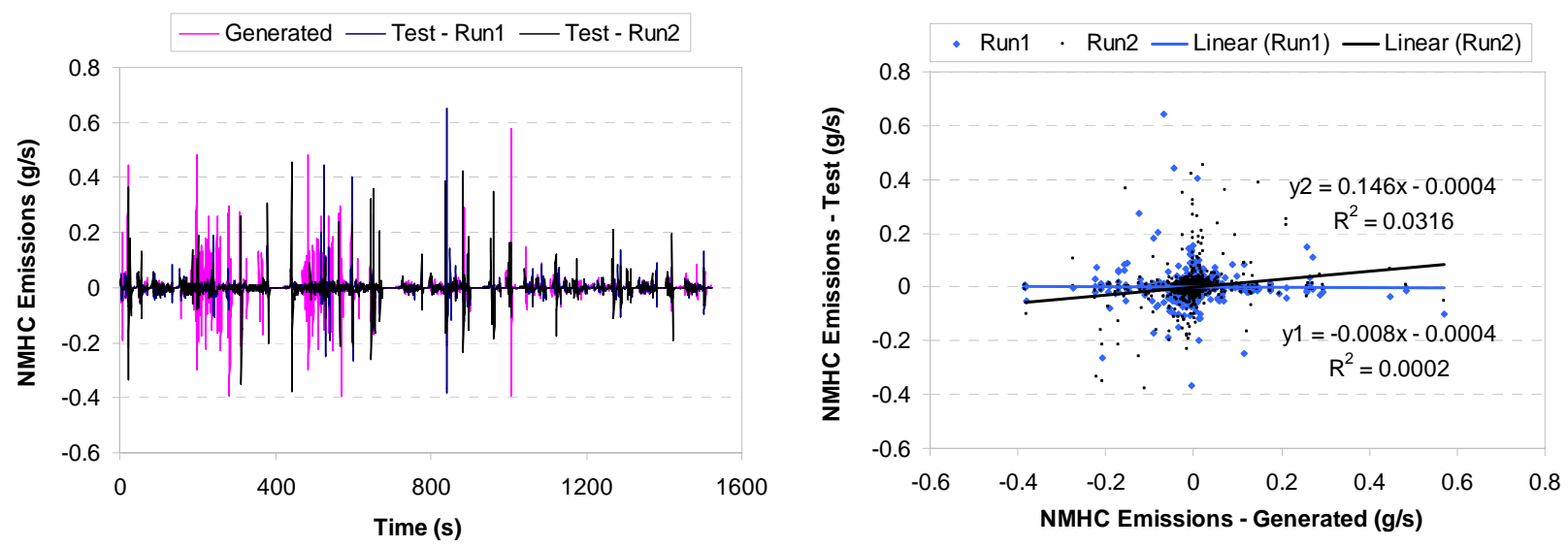

Figure 5.21 NMHC Rates from Emissions Testing and Cycle Generation of VC_637 from a MY 2008 CNG Bus

\subsubsection{Hybrid Bus}

This subsection discusses a MY 2008 hybrid bus tested over the same set of standard cycles and virtual cycles as tested for the CNG bus. To be noted, state of charge (SOC) of the hybrid bus was controlled to be zero change for all tests compliant with SAE J2711 [51]. In this way, it could be certain that the overall energy for the cycle test was acquired from fuel rather than the battery. However, during cycle generation, this requirement might not be met since zero SOC change condition was not applied to continuous data during the test. The SOC implications of the cycle generation method could not be accounted for because SOC data were not continuously recorded over the cycle.

Table 5.4 gives integrated values from emission testing and cycle generation while Figure 5.22 provides their corresponding relative differences. Compared to the CNG bus, the hybrid bus presented significantly lower CO and HC emissions but higher NOx and fuel economy. Table 5.4 shows negative values for $\mathrm{HC}$ emissions and it might indicate that $\mathrm{HC}$ levels were too low to be detected by the emissions analyzers. As described previously, this hybrid bus was equipped with a DPF and DOC which resulted in substantial reduction in CO and HC emissions.

Similar to the CNG bus, the hybrid bus showed small relative differences in $\mathrm{CO}_{2}$ and fuel economy while significantly larger differences in terms of $\mathrm{CO}$ and $\mathrm{HC}$ between emissions testing 
and cycle generation. It was noted that unlike the CNG bus the hybrid bus presented small relative differences (within 2\%) in terms of NOx emissions.

Table 5.4 Results of Emissions Testing and Cycle Generation of Selected Virtual Cycles from a MY 2008 Hybrid Bus

\begin{tabular}{lccccc}
\hline \multicolumn{5}{c}{ Emissions Testing } \\
\hline & $\mathbf{C O}$ (g/mile) & $\mathbf{C O}_{\mathbf{2}}$ (g/mile) & NOx (g/mile) & HC (g/mile) & Fuel Economy (mpg) \\
\hline VC_274 & 0.10 & 2052.01 & 5.46 & -0.01 & 5.06 \\
VC_637 & 0.03 & 1753.32 & 5.18 & -0.01 & 5.91 \\
VC_28 & 0.22 & 2868.40 & 10.72 & -0.02 & 3.60 \\
\hline \multicolumn{5}{c}{ Cycle Generation } \\
\hline VC_274 & CO (g/mile) & $\mathbf{C O}_{\mathbf{2}}$ (g/mile) & NOx (g/mile) & HC (g/mile) & Fuel Economy (mpg) \\
VC_637 & 0.19 & 1954.30 & 5.38 & -0.01 & 5.30 \\
VC_28 & 0.13 & 1745.40 & 5.09 & 0.00 & 5.93 \\
\hline
\end{tabular}

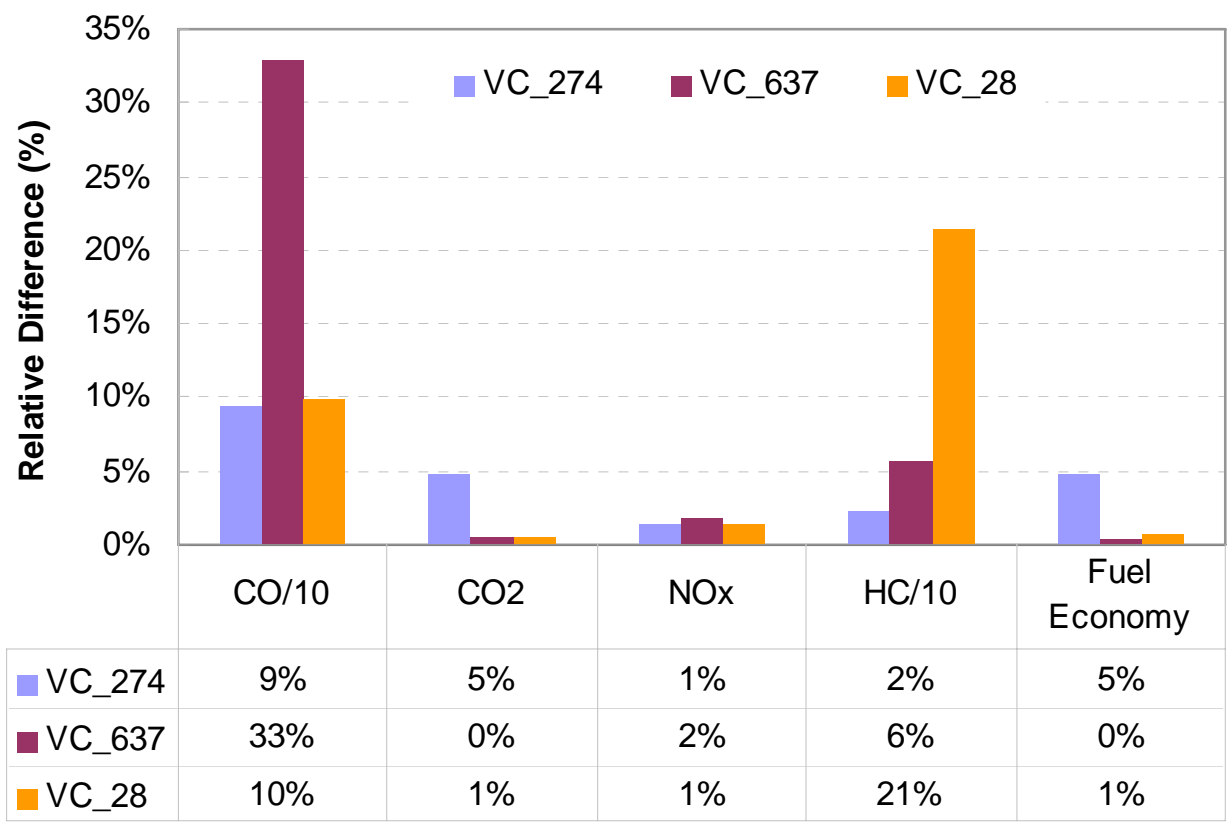

Figure 5.22 Relative Difference between Emissions Testing and Cycle Generation of Selected Virtual Cycles from a MY 2008 Hybrid Bus

Figure 5.23 shows target and actual speed as well as fuel consumption and $\mathrm{CO}_{2}$ rates from emissions testing and cycle generation of the VC_274 cycle while Figure 5.24 shows CO, HC and NOx emissions rates. It is evident that the driver could closely follow the target cycle with slope and $R$ squared values both above 0.99. Compared to the CNG bus on the same cycle ( $R$ squared of 0.88 ), the parity plots of fuel consumption and $\mathrm{CO}_{2}$ appear more scattered ( $R$ squared of 0.80 ) but still show high accuracy of cycle generation with slopes of above 0.94. At 
higher speed (above $40 \mathrm{mph}$ ), more deviation was observed from the parity plot of speed-time trace which might result in larger differences between emissions testing and cycle generation in terms of fuel consumption and $\mathrm{CO}_{2}$ rates and show as the more scattering points.
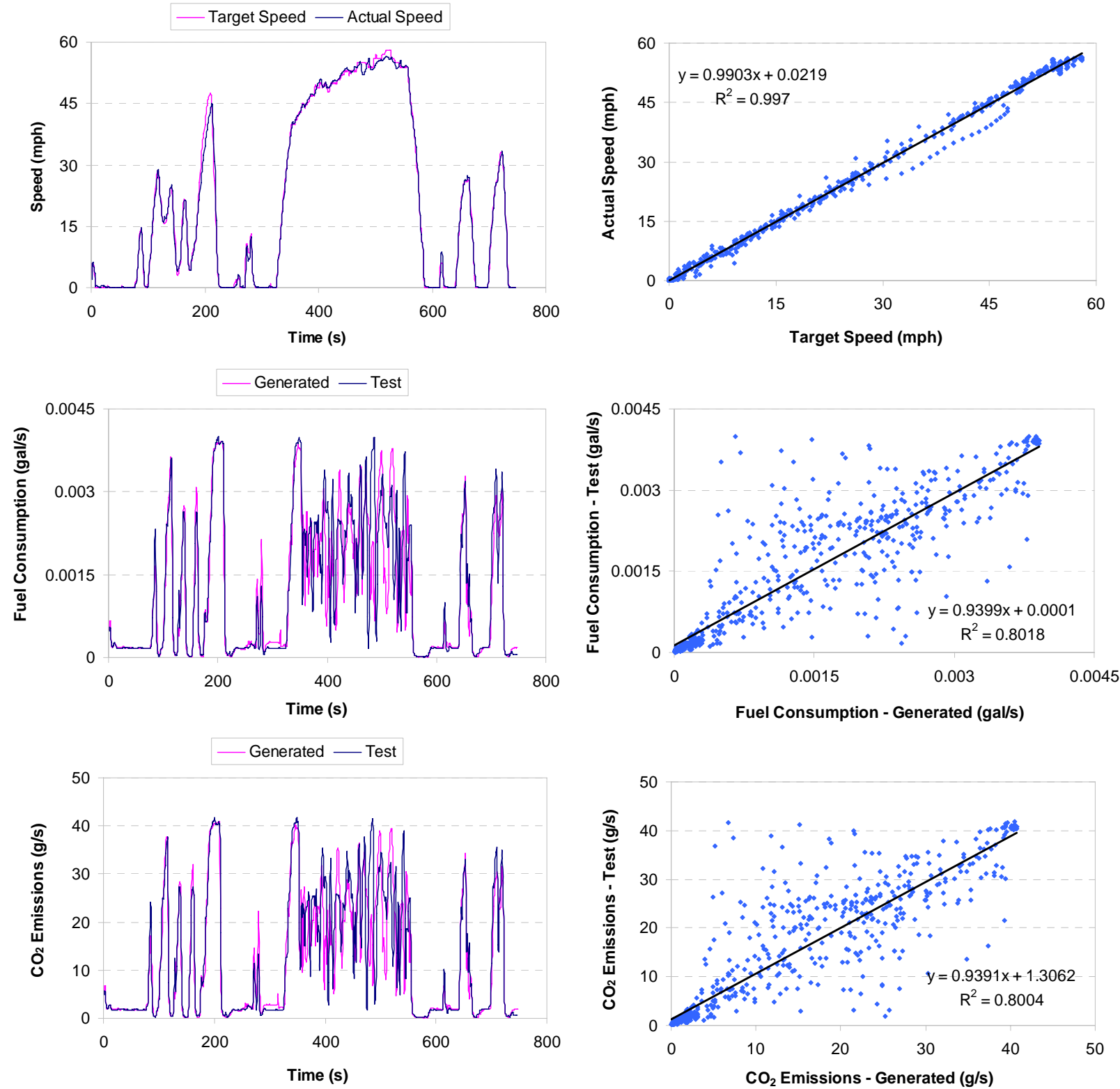

Figure 5.23 Target and Actual Vehicle Speed, Fuel Consumption and $\mathrm{CO}_{2}$ Rates from Emissions Testing and Cycle Generation of VC_274 from a MY 2008 Hybrid Bus

Figure 5.24 shows near zero emission level of both $\mathrm{CO}$ and $\mathrm{HC}$ and some HC rates were below the detectable level showing as negative values. A few spikes were observed at high acceleration points. Small values were found in terms of slopes and $R$ squared but overall both emissions testing and cycle generation demonstrated nearly zero emissions of CO and HC. 
Unlike the CNG bus discussed previously, the hybrid bus showed much better values with respect to slopes and $R$ squared values for NOx emissions and illustrated that cycle generation could accurately represent the NOx emissions test results on the cycle.
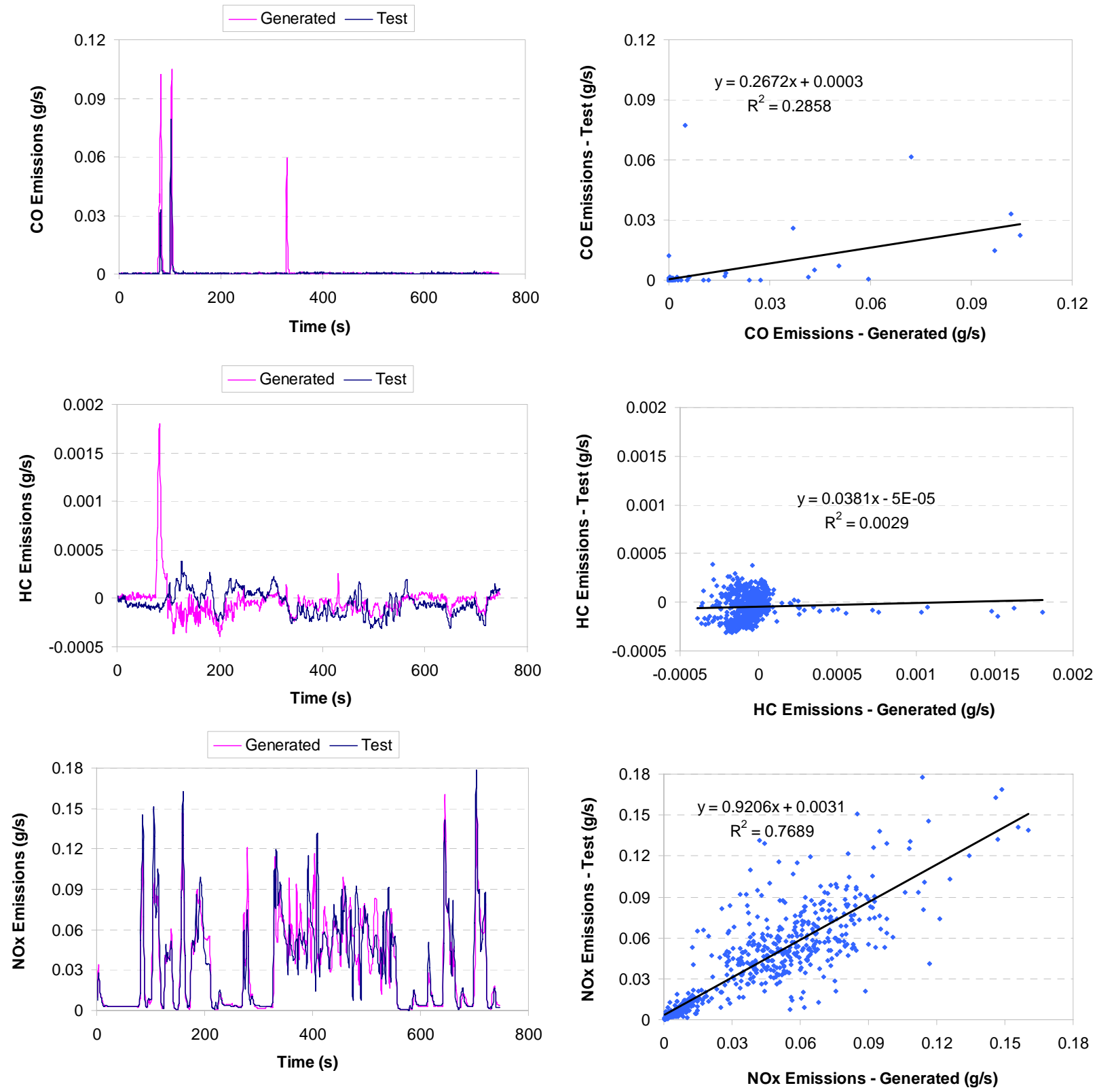

Figure 5.24 CO, HC and NOx Rates from Emissions Testing and Cycle Generation of VC_274 from a MY 2008 Hybrid Bus

Regarding the other two virtual cycles, Figure B-5 - Figure B-8 in Appendix B illustrate similar results to those of the VC_274 cycle discussed above. The regression summary of parity plots was provided in Table 5.5 . 
Table 5.5 Regression Summary from Parity Plots of Selected Virtual Cycles from a MY 2008 Hybrid Bus

\begin{tabular}{llcccccc}
\hline & & Speed & CO & CO $_{2}$ & NOx & HC & Fuel Consumption \\
\hline \multirow{2}{*}{ VC_274 } & Slope & 0.99 & 0.27 & 0.94 & 0.92 & 0.04 & 0.94 \\
& $\mathrm{R}^{2}$ & $>0.99$ & 0.29 & 0.80 & 0.77 & $<0.01$ & 0.80 \\
\hline \multirow{2}{*}{ VC_637 } & Slope & 0.99 & $<0.01$ & 0.90 & 0.87 & 0.83 & 0.90 \\
& $\mathrm{R}^{2}$ & $>0.99$ & $<0.01$ & 0.78 & 0.63 & 0.55 & 0.79 \\
\hline \multirow{2}{*}{ VC_28 } & Slope & 0.96 & 0.02 & 0.93 & 0.93 & $<0.01$ & 0.93 \\
& $\mathrm{R}^{2}$ & 0.99 & $<0.01$ & 0.90 & 0.78 & $<0.01$ & 0.90 \\
\hline
\end{tabular}

\subsection{Summary}

In total, 368 virtual cycles as well as their emissions and fuel economy data were generated. Generated points followed the same trends as reference target cycle points. In addition, the cycle generator severed its purpose to fill some sparse areas in cycle parameter spaces. Some areas could not be filled by generated cycle points and might indicate impractical operation profiles of transit buses.

Curve fitting showed that average speed had a dominant role to estimate emissions and fuel economy, which provided insight into how to effectively select polynomial terms during the development of backbone models based on two cycle parameters. However, wider variations at certain average speeds reinforced that average speed based single cycle parameter emission models might not be sufficiently accurate to estimate response variables.

Emissions testing verified that selected virtual cycles were realistic in that the driver could closely follow the target speed-time traces with high repeatability.

Integrated values from emissions testing for fuel economy and $\mathrm{CO}_{2}$ emissions could be accurately represented by the cycle and emission generation method within 1\% and 2\% differences respectively for the CNG bus and within 5\% for the hybrid bus. For both CNG and hybrid buses, second-by-second fuel consumption and $\mathrm{CO}_{2}$ rates could be relatively accurately (slope values of 0.89 or above) and relatively precisely ( $R$ squared of 0.78 or above) represented by the generation method.

Preliminary results did not show that $\mathrm{HC}$ and $\mathrm{CO}$ emissions rates and integrated values could be accurately represented by the cycle and emissions generation method for both CNG and hybrid buses. 
The stoichiometric CNG bus with a TWC presented a low level of NOx emissions. For the CNG bus, NOx emissions rates and integrated values could be moderately represented at average speed of above $8 \mathrm{mph}$ by the cycle generation method. At lower average speed with higher percentage idle and number of stops per mile, cycle generation could result in larger errors. Compared to the CNG bus, the hybrid bus presented significantly higher NOx emissions and NOx emissions could be more accurately and precisely presented by cycle generation results.

Experimental results of selected virtual cycles demonstrated that fuel economy, $\mathrm{CO}_{2}$ and NOx emissions might not be sensitive to microtrip history. Limited repeat tests further demonstrated that the larger deviations of $\mathrm{HC}$ and $\mathrm{CO}$ between emissions testing and cycle generation might be attributed to not only the changes of microtrip history from cycle generation but also their high sensitivity to transient features of driving condition.

In summary, cycle and emissions generation could be employed to transit buses tested over the limited number of cycles and substantially expand database for emissions and fuel economy modeling. 


\section{IBIS BACKBONE MODELING}

Five cycle metrics were determined to be most influential parameters affecting emissions and fuel economy. In addition, the above analyses determined the major role of average speed as an independent variable in a model as well as suggested adding other selected cycle metrics could improve the results. Based on these suggestions, this chapter is focused on the development of IBIS backbone models.

\subsection{Emission Data Preparation}

The WVU emissions database summarized testing results from over 400 transit buses, most of which were used in this study. To develop the backbone models, eight candidate buses were selected to represent similar buses in their specific MY groups and these buses were tested on a variety of different chassis dynamometer test cycles with respect to emissions and fuel consumption. Vehicle parameters of the selected eight backbone buses are summarized in Table 6.1. The selected vehicles included diesel, CNG and hybrid diesel-electric transit buses.

The model years of these buses ranged from 2000 to 2008 and represented three MY groups. For older vehicles before MY 2007, emissions testing procedures followed the CFR 40, Part 86, Subpart N [60] where applicable to chassis dynamometer testing. For the newer vehicles beyond MY 2007, emissions testing and calculation followed the procedures of CFR 40, Part 1065 [79].

Emissions testing data needed to be processed for backbone modeling. For each cycle, continuous emissions data were time-aligned with vehicle speed to account for the time delay between the emissions and vehicle speed which resulted from the travelling time of exhaust emissions through the dilution tunnel and the response time of the exhaust gas analyzers [82]. For each test run, the time shifts were determined using the highest cross-correlation coefficients between emissions data and the target speed-time trace [98]. In addition, dispersion could affect continuous data [92]. Then, the diesel equivalent fuel consumption rates were computed based on carbon balance using fuel properties and measured carbon emissions. The above procedures were applied to transit buses with model years before 2007. 
Table 6.1 Vehicle Parameters of the Eight Selected Backbone Buses

\begin{tabular}{|c|c|c|c|c|c|c|c|c|}
\hline Backbone Model & $\begin{array}{l}\text { Diesel } \\
\text { MY } \\
\text { Group IV }\end{array}$ & $\begin{array}{l}\text { Diesel } \\
\text { MY } \\
\text { Group V }\end{array}$ & $\begin{array}{l}\text { Hybrid } \\
\text { MY } \\
\text { Group V }\end{array}$ & $\begin{array}{l}\text { Hybrid } \\
\text { MY } \\
\text { Group V }\end{array}$ & $\begin{array}{l}\text { CNG MY } \\
\text { Group V }\end{array}$ & $\begin{array}{l}\text { CNG MY } \\
\text { Group V }\end{array}$ & $\begin{array}{l}\text { CNG MY } \\
\text { Group } \\
\text { VI }\end{array}$ & $\begin{array}{l}\text { Hybrid } \\
\text { MY } \\
\text { Group VI }\end{array}$ \\
\hline Bus Type & Diesel & Diesel & Hybrid & Hybrid & CNG & CNG & CNG & Hybrid \\
\hline $\begin{array}{l}\text { WVU Bus ID } \\
\text { Number }\end{array}$ & 2094 & 6150 & 204 & 6003 & 2640 & 2503 & 5420 & 6315 \\
\hline $\begin{array}{l}\text { Vehicle } \\
\text { Manufacturer }\end{array}$ & Orion & $\begin{array}{l}\text { New } \\
\text { Flyer }\end{array}$ & Orion & New Flyer & Orion & Orion & NABI & New Flyer \\
\hline Vehicle Model Year & 2000 & 2006 & 2006 & 2005 & 2005 & 2005 & 2008 & 2008 \\
\hline $\begin{array}{l}\text { Gross Vehicle } \\
\text { Weight Rating } \\
\text { (GVWR) (lb.) }\end{array}$ & 42,540 & 40,600 & 42,540 & 40,600 & 42,540 & 42,540 & 68,540 & 42,540 \\
\hline $\begin{array}{l}\text { Vehicle Curb Weight } \\
\text { (lb.) }\end{array}$ & 28,800 & 30,280 & 33,440 & 31,780 & 32,320 & 32,300 & 47,030 & 33,660 \\
\hline $\begin{array}{l}\text { Vehicle Length } \\
\text { (feet) }\end{array}$ & 40 & 40 & 40 & 40 & 40 & 40 & 60 & 40 \\
\hline Transmission Type & Auto & Auto & Hybrid & Hybrid & Auto & Auto & Auto & Hybrid \\
\hline $\begin{array}{l}\text { Transmission } \\
\text { Configuration }\end{array}$ & 4 Speed & 4 Speed & Series & Parallel & 4 Speed & 4 Speed & 5 Speed & Parallel \\
\hline Engine Type & $\begin{array}{l}\text { Detroit } \\
\text { Diesel } \\
\text { S50 }\end{array}$ & $\begin{array}{l}\text { Cummins } \\
\text { ISM } 280\end{array}$ & $\begin{array}{l}\text { Cummins } \\
\text { ISB260H }\end{array}$ & $\begin{array}{l}\text { Cummins } \\
\text { ISL } 280\end{array}$ & $\begin{array}{l}\text { John Deere } \\
6081 \mathrm{H}\end{array}$ & $\begin{array}{l}\text { Cummins } \\
\text { CG-280 }\end{array}$ & $\begin{array}{l}\text { Cummins } \\
\text { ISL-G320 }\end{array}$ & $\begin{array}{l}\text { Cummins } \\
\text { ISL } 280 \mathrm{H}\end{array}$ \\
\hline Engine Model Year & 2000 & 2006 & 2006 & 2005 & 2005 & 2005 & 2008 & 2008 \\
\hline $\begin{array}{l}\text { Engine } \\
\text { Displacement (Liter) }\end{array}$ & 8.5 & 10.8 & 5.9 & 8.3 & 8.1 & 8.3 & 8.9 & 8.9 \\
\hline $\begin{array}{l}\text { Engine Rated Power } \\
\text { (hp) }\end{array}$ & 275 & 280 & 260 & 280 & 280 & 280 & 320 & 280 \\
\hline Primary Fuel & ULSD1 & ULSD1 & $\begin{array}{l}\text { WCULSD } \\
1\end{array}$ & ULSD1 & CNG & CNG & CNG & ULSD1 \\
\hline $\begin{array}{l}\text { Particulate Trap } \\
\text { Manufacturer }\end{array}$ & & & Nelson & Engelhard & & & & DPF \\
\hline $\begin{array}{l}\text { Catalytic Converter } \\
\text { Manufacturer }\end{array}$ & & Nelson & Nelson & & $\begin{array}{l}\text { Catalytic } \\
\text { Converter }\end{array}$ & $\begin{array}{l}\text { Catalytic } \\
\text { Converter }\end{array}$ & $\begin{array}{l}\text { Catalytic } \\
\text { Converter }\end{array}$ & DOC \\
\hline
\end{tabular}

In terms of time-alignment evaluation, CFR 40 Part 1065 [79] suggested that the $t_{50}$ times of the gas analyzer signals be recorded [79]. At the $t_{50}$ point, each analyzer has passed the midpoint of its response. The target of the time-alignment evaluation is to ensure that all $t_{50}$ points occur at the time. During the emissions testing of MY 2008 transit buses in this study, eight seconds were determined as the shift time for all analyzer signals. During data processing, all continuous emissions data were shifted eight seconds ahead to offset the response time of the emission analyzers.

If repeat tests were available for certain cycles, then the results from all repeats were averaged. After data processing for each of the tested cycles, the standard cycle emissions database was constructed which consisted of tested cycles with their target speed-time traces and 
second-by-second emissions and fuel consumption rates. Then the next step was to obtain the emissions data from newly generated virtual cycles, as shown in Figure 5.3. After the cycle generation process, the emissions database for backbone modeling was significantly expanded and include not only the data from a limited number of actual tested cycles but also those from over 350 virtual cycles generated.

\subsection{Methodology}

Two diesel buses were selected to develop two backbone models and represent MY 1998 - 2002 (designated as MY group IV) and MY 2003 - 2006 (designated as MY group V) diesel buses. Two CNG buses with different engine manufactures were selected to develop one backbone model representing MY 2003 - 2006 (designated as MY group V) CNG buses. For MY 2007 to 2009 (designated as MY group VI), one MY 2008 60-ft CNG bus was selected as the candidate for the backbone model. A 60-ft bus based backbone model might not represent 40-ft buses due to different weight and power but the model could be adjusted with a power to weight ratio the applied to 40-ft buses. One parallel and one series diesel-electric hybrid bus were selected to develop the composite backbone model for MY 2003 - 2006 (designated as MY group V) hybrid buses. For MY 2007 to 2009, one MY 2008 parallel diesel-electric hybrid bus

was selected as the candidate for the backbone model. In total, six backbone models based on the eight tested buses shown in Table 6.1 were developed with two models for each type of fuel or powertrain technology used by the vehicles.

Previous correlation analysis showed that instead of a linear relationship, a non-linear relationship should be expected between emissions and cycle parameters. Polynomial regression models were used to build backbone models.

The five selected cycle parameters discussed in Chapter 4 were used as backbone model inputs. The primary modeling strategy was to use average speed (with idle) with one of the other four selected cycle parameters as pairs of independent variables. The other four cycle parameters were percentage idle, number of stops per mile, standard deviation of vehicle speed (with idle) and kinetic intensity. For example, if average speed, percentage idle, and stops per mile are available for IBIS users as inputs, the model would fit emissions using one sub-model based on average speed and percentage idle, and another based on average speed and stops per mile. The results from the two polynomial sub-models were averaged to obtain the outputs of the backbone 
model. If only average speed was available to IBIS users, then the model could use the input average speed combined with the default value from the WMATA cycle for percentage idle. This was an approximate approach but it was reasonable considering the dominant role of average speed as discussed in Chapter 5. The general modeling form as a function of polynomial pairs can be expressed as follows:

$$
y=a+\sum_{i=1}^{n} b_{v, i} x_{v}^{i}+\sum_{j=0}^{n} c_{u, j} x_{u}^{j}+\sum_{l, m=1}^{n} d_{l, m} x_{v}^{l} x_{u}^{m}+\varepsilon
$$

Equation 6.1

where $y$ corresponds to distance-specific emission values or fuel economy while $x_{v}$ is average speed and $x_{u}$ is another available cycle parameter. The degree of polynomial is input by the user as $n$ and the sum of $l$ and $m$ must be no more than $n$. The final estimated value of the model was computed as the average of all available polynomial sub-models with pair cycle parameters as follows:

$$
y_{f}=\frac{\sum_{e=1}^{k-1} y_{e}}{k-1}, \quad k \leq 5
$$

Equation 6.2

where $k$ corresponds to the total number of available cycle parameters input by the user and $y_{e}$ corresponds to $y$ in Equation 6.1 which is the estimate from each of polynomial pair submodels. For example, the flowchart of a general backbone model development for NOx emissions could be illustrated as Figure 6.1. For the other emissions or fuel economy, the same flowchart could be applied. It shows that four sub-models are used to develop the final combined model, and average speed is a mandatory input.

The reason that average speed was selected as the mandatory input was attributed to its importance and easy for users to obtain for their fleet. As a result, four sub-models were developed for a final combined model. When the estimates from each sub-model were averaged, it was assumed that each sub-model shared equal weight in the predicted values of the final model. Equal weight was reasonable since each sub-model used average speed as major predictor even though the second parameter might have relative minor impact.

Alternatively, a final model could be based on any combination of the five selected cycle parameters. In this case, 30 sub-models could be developed for one final backbone model, making the modeling complicated. Polynomial sub-models based on the combination of three cycle parameters were preliminarily evaluated and compared with pairs [78]. It showed the first 
one was more accurate than the later. However, it was not easy to obtain three cycle parameters for the users. Additionally, it resulted in 4-dimensional spaces thus it was difficult to interpret the results. Using averaged speed based pair models was simpler while it could still predict the results with average percentage errors below $12 \%$ with only second degree polynomials [78].

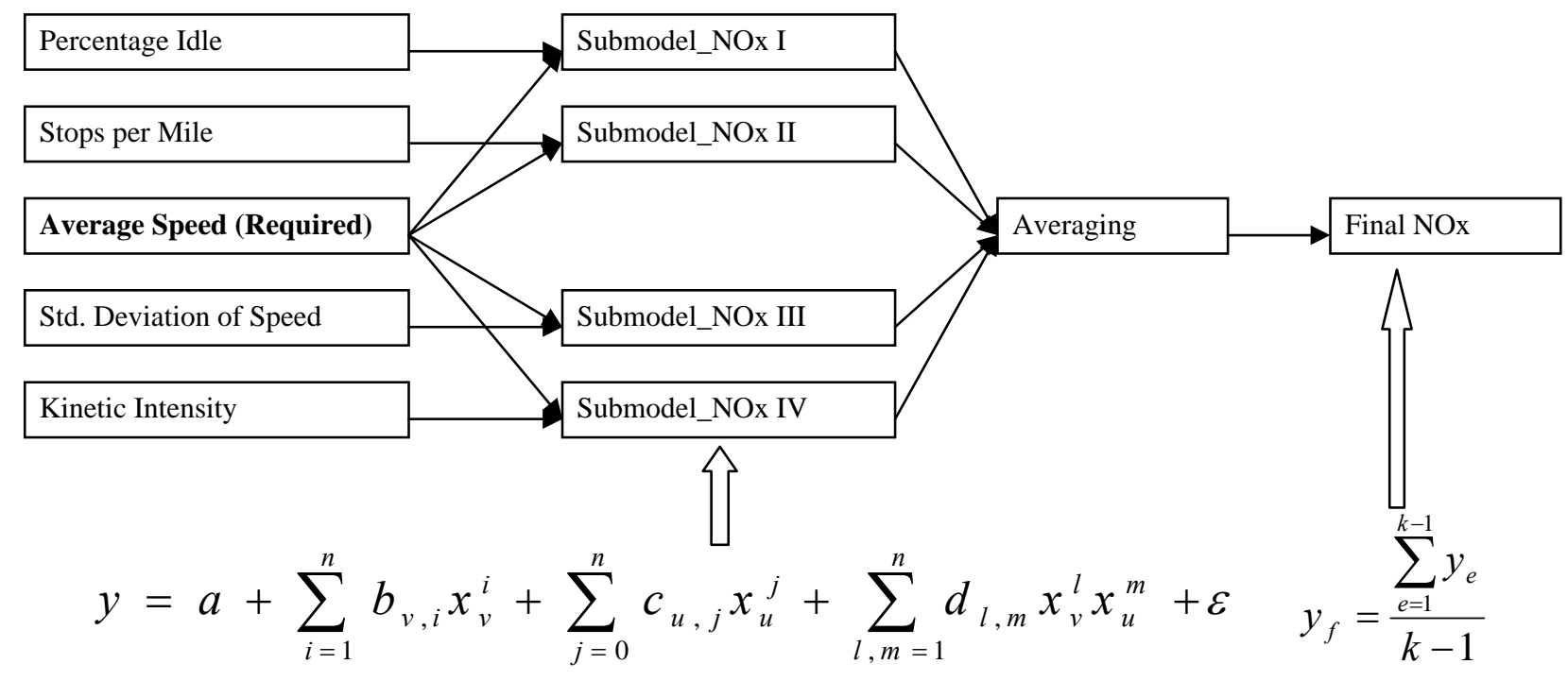

Figure 6.1 A Flowchart for General Backbone Modeling of NOx Emissions

\subsection{Development of Backbone Models}

As a case study, this section was focused on developing the backbone models for MY 2008 transit buses. The two buses for backbone models are CNG \#2 and Hybrid \#2, as listed in Table 6.1. The chassis dynamometer emissions testing was conducted at WMATA, Lanover, MD using the WVU TransLab which compiled with the CFR 40, Part 1065 [79]. Multiple newer (after MY 2007) transit buses were tested and two of them were selected for modeling. They included one MY 2008 60-ft stochiometric CNG bus and another MY 2008 40-ft diesel hybridelectric bus and both were tested over 16 cycles. The CNG bus was equipped with the three-way catalytic converter and the hybrid bus was equipped with an active diesel particulate filter (DPF) and diesel oxidation catalysts (DOC). Repeat runs were conducted for some cycles. The tested cycles used to generate virtual cycles for modeling were summarized in Table 2.4.

An interactive tool with GUI was developed using MATLAB ${ }^{\circledR}$ to assist in determining the candidate polynomial sub-models for backbone modeling [78]. The tool could not be automated to obtain the final sub-models and it required a significant amount of human 
involvement. The mean percent error in combination with visual evaluation was used to evaluate the fit of the models. A model could be overfitted while showing the smallest error. The general selection procedure started by selecting the possible highest order of the polynomial model which could show the lowest mean percent error. In this case, usually six or seven was selected as the degree of the polynomial models [78]. To reduce the terms of the model, the following procedures with priority from high to low were conducted:

- The higher order terms of the second parameter were removed. This was mainly due to the dominant role of the average speed.

- The interaction terms composed of both cycle parameters were also a high priority for removal, since they represented the shared effect from both parameters and might adversely affect the modeling.

- The terms showing relatively smaller coefficients (compared to their values) could be removed later, which might indicate they were not significant for modeling.

- This step might include the removal of higher orders of average speed as well since higher orders of the polynomials could result in overfit or overshoot. An ideal candidate sub-model could be a surface which goes between the cycle points in the space and with a lower value of the mean percent error.

Depending on users, different procedures from above could be performed to reduce the terms. Based on the quality of the available emissions data, two types of sub-models were developed for the two buses, including models based on both of tested and generated cycle points, and models based on only tested cycles associated with repair algorithms. Once the sub-models were determined, they were implemented into a model check tool [78] to demonstrate and further visually evaluate them in the 3-dimension (3-D) space. Sub-models were plotted on the previously determined domains as shown in Figure 5.4 - Figure 5.7. The results are shown in the following sections.

\subsubsection{Models Based on the Expanded Database}

This type of models was developed for fuel economy, $\mathrm{CO}_{2}, \mathrm{HC}$, and $\mathrm{CO}$ emissions of the CNG bus and for fuel economy, $\mathrm{CO}_{2}$, and NOx emissions of the hybrid bus.

The selected pair sub-models for each dependent variable mentioned above were illustrated as Figure 6.2 - Figure 6.6 for the MY 2008 CNG bus. Figure 6.2 shows the fuel 
economy sub-model as a function of average speed and percentage idle. Figure 6.2 (b) and (c) represent the projections of the 3-D model to the plane of fuel economy vs. average speed, and the plane of fuel economy vs. percentage idle, respectively. They showed the ranges of the fuel economy from below 0.5 to 4 mpg. Figure 6.2 (b) demonstrates that fuel economy increases with increasing average speed and reaches $4 \mathrm{mpg}$ at approximate $45 \mathrm{mph}$. It also shows small variations of fuel economy with the average speed. Variations of up to $0.75 \mathrm{mpg}$ occurred at average speeds ranging from 20 to $30 \mathrm{mph}$. This indicated that average speed alone might be used to approximately estimate fuel economy.

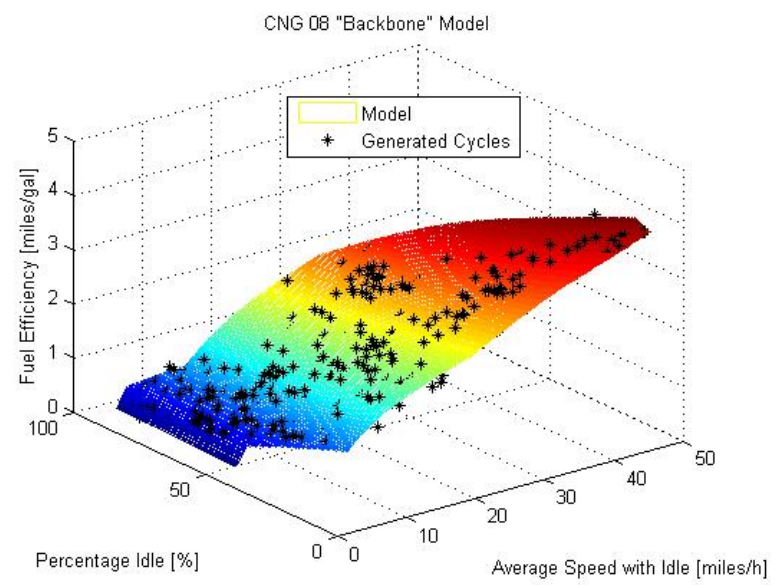

(a) Sub-model of average speed with percentage idle

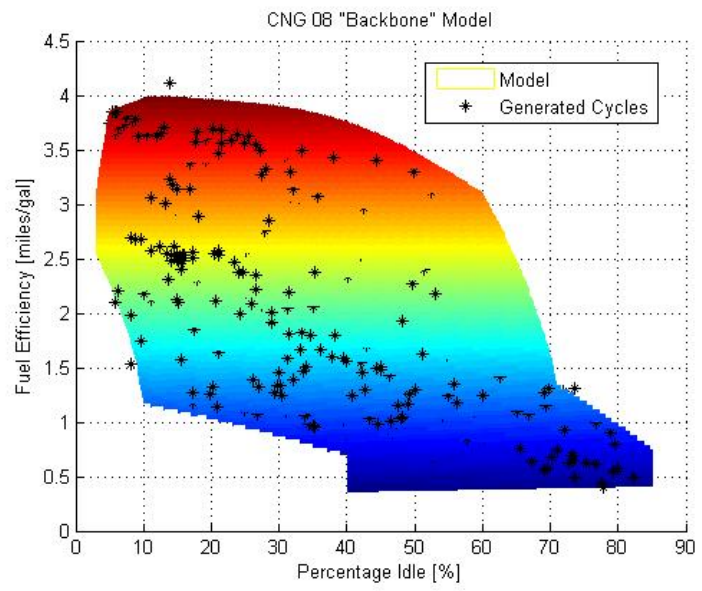

(c) Projection Sub-model (a) to the plane of fuel economy vs. percentage idle

Figure 6.2 Fuel Economy Backbone Sub-Model Based on Average Speed and Percentage Idle for a MY 2008 CNG Bus

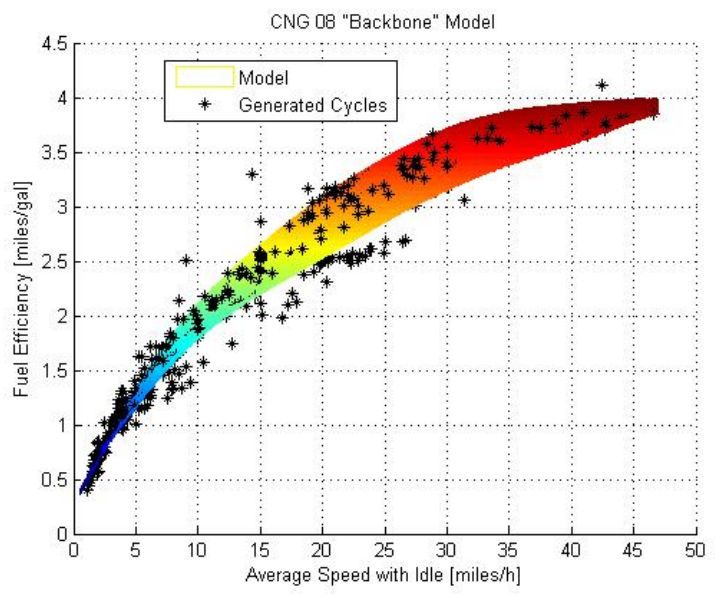

(b) Projection Sub-model (a) to the plane of fuel economy vs. average speed 
In contrast to average speed, Figure 6.2 (c) shows that fuel economy varies significantly with percentage idle and the projected area covers nearly all of the cycle points. A good fit usually indicates that the fitted curve lies among or closes to the cycle points. As an example, Figure 6.2 demonstrates that the sub-model was a good fit. If a higher order polynomial were selected, the model might show overshoots or significant oscillations in some areas, indicating that overfitting occurred. In contrast, a model might be underfit if the surface is too flat with increased percent errors. To avoid these two situations, error analysis in combination with visual evaluation was conducted to select a good 3-D fitting model. The error analysis of the model is provided later in this chapter.

The other fuel economy sub-models are shown as Figure 6.3, including using average speed with each of the other cycle parameters to fit the model. As shown in Figure 6.2, some cycle points are above or on these curves while others are below, indicating the selected models have a good fit to the data.

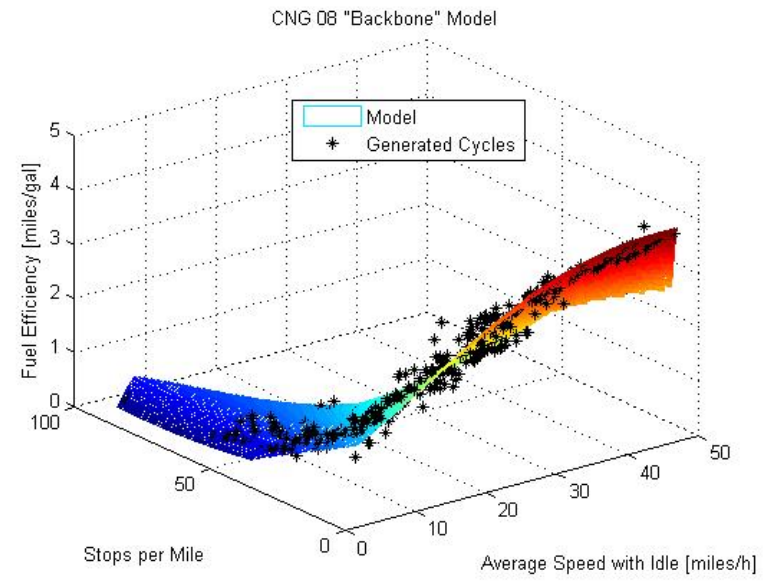

(a) Sub-model of average speed with stops/mile

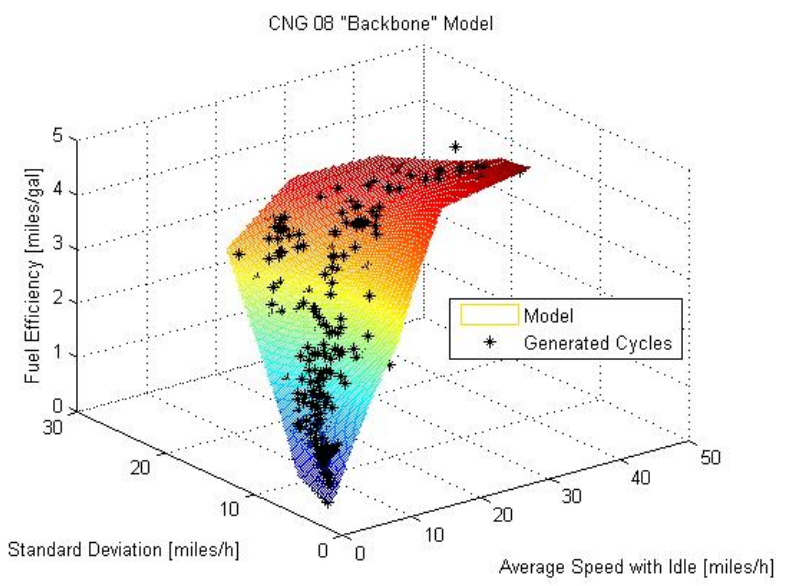

(b) Sub-model of average speed with standard deviation 


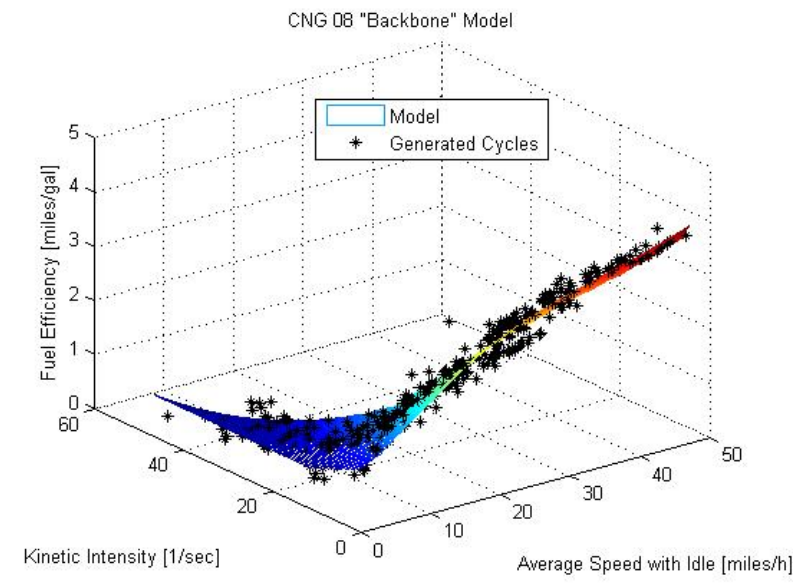

(c) Sub-model of average speed with kinetic intensity

Figure 6.3 Fuel Economy Backbone Sub-Models Based on Average Speed and Other Parameters for a MY 2008 CNG Bus

For $\mathrm{CO}_{2}$, $\mathrm{CO}$ and $\mathrm{HC}$ from the same CNG bus, the selected models are shown as Figure 6.4 - Figure 6.6. Figure 6.4 shows two of $\mathrm{CO}_{2}$ sub-models, one as a function of average speed with percentage idle, and the other as a function of average speed with kinetic intensity.
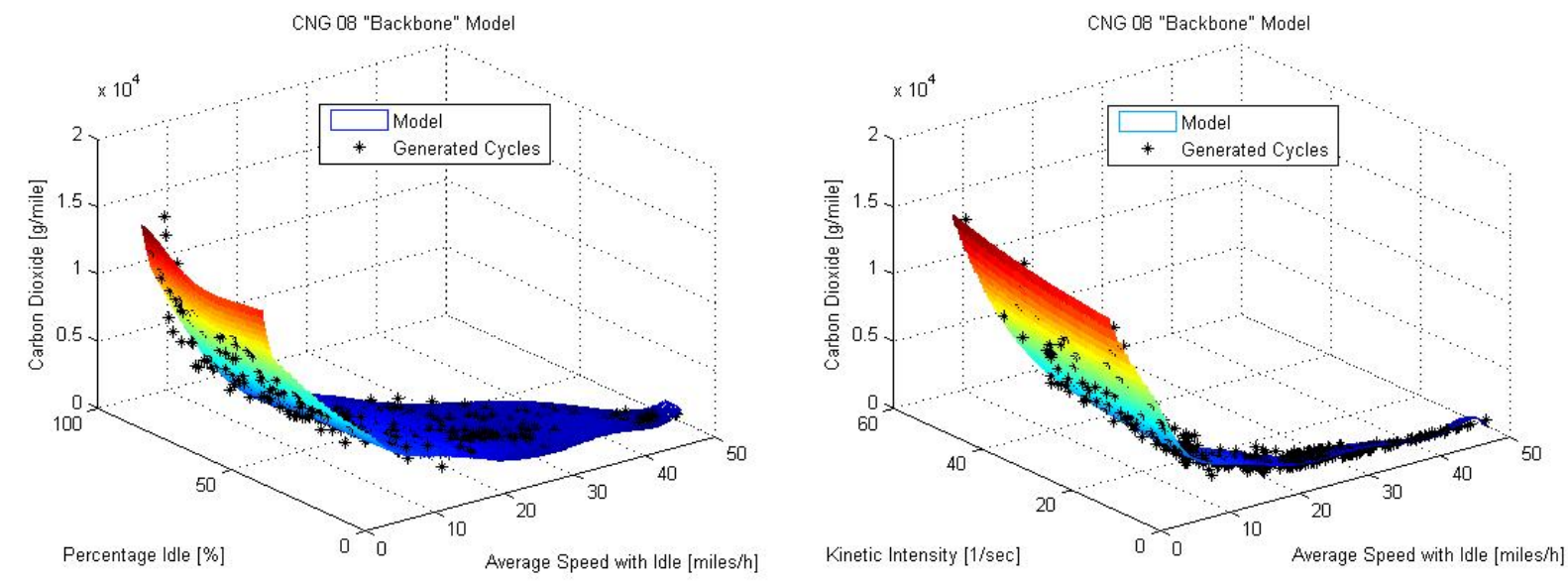

Figure 6.4 $\mathrm{CO}_{2}$ Backbone Sub-Models in Terms of Average Speed with Percentage Idle and Kinetic Intensity Based on a MY 2008 CNG Bus 
Figure 6.5 and Figure 6.6 look similar, while the first one represents one of the CO submodels and the later represents one of the HC sub-models. Both models were based on average speed with stops per mile.

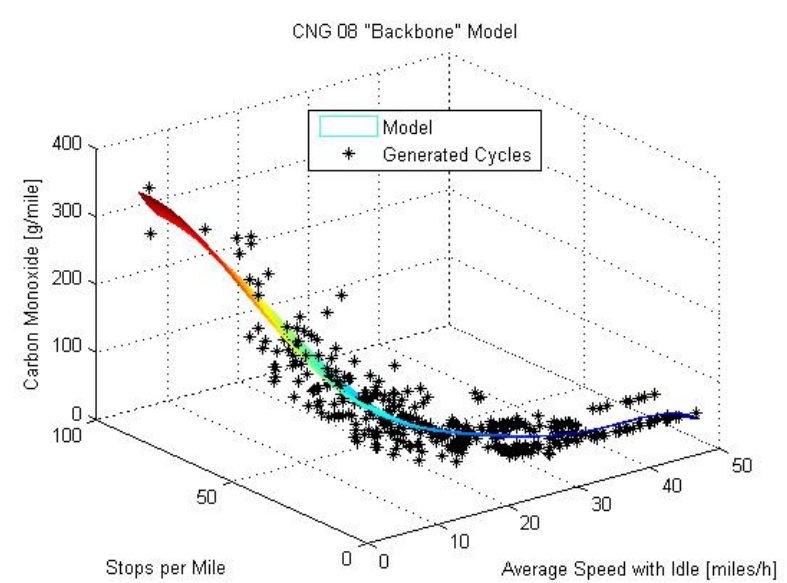

Figure 6.5 CO Backbone Sub-Models in Terms of Average Speed with Stops/mile Based on a MY 2008 CNG Bus

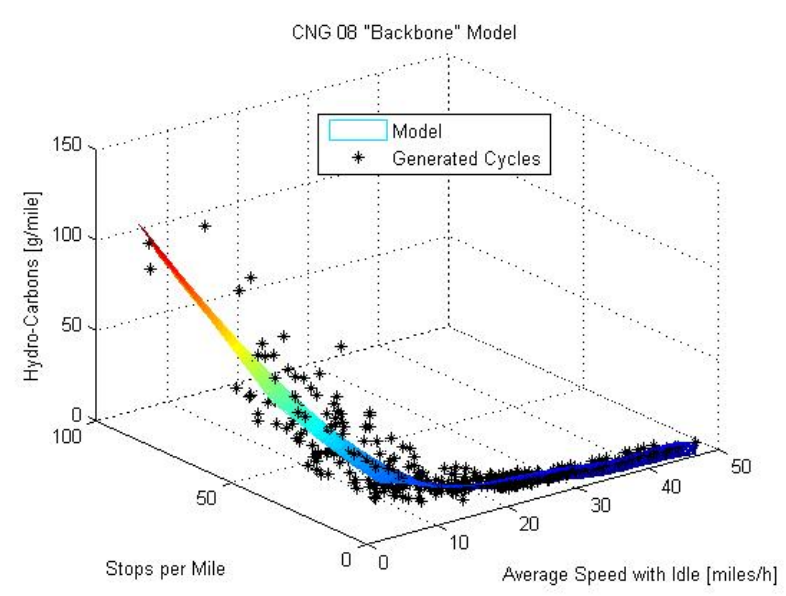

Figure 6.6 HC Backbone Sub-Models in Terms of Average Speed with Stops/mile Based on a MY 2008 CNG Bus

The similarity between the two figures indicated the correlation between $\mathrm{CO}$ and $\mathrm{HC}$. Both emissions are sensitive to the transient features of driving condition and both are the reactants of the three-way catalytic converter equipped on the bus. In terms of NOx, NMHC and $\mathrm{PM}$, they were discussed in the later section.

Figure 6.7 presents three of the selected sub-models for fuel economy, $\mathrm{CO}_{2}$ and $\mathrm{NOx}$, respectively, from the MY 2008 hybrid bus. The top two figures show the fuel economy and it is up to $6.5 \mathrm{mpg}$ for the hybrid bus which is significantly better than that from the above CNG bus. There were other sub-models for the same dependant variable which were determined by other pairs of the cycle parameters and were not presented herein. Compared with the CNG bus above, the fuel economy of the hybrid bus had larger variations with average speed. It was noted that the surfaces of fuel economy and $\mathrm{CO}_{2}$ in Figure 6.7 present the similar shape as those from the CNG bus as shown in Figure 6.2 and Figure 6.4, respectively. In fact, in terms of fuel economy and $\mathrm{CO}_{2}$, the corresponding sub-models fitted by the same pairs of cycle parameters look similar between the two buses. This might indicate the correlations are quite consistent for both fuel economy and $\mathrm{CO}_{2}$ with cycle parameters and these relationships might not change significantly for a variety of transit buses operated at different road conditions. 

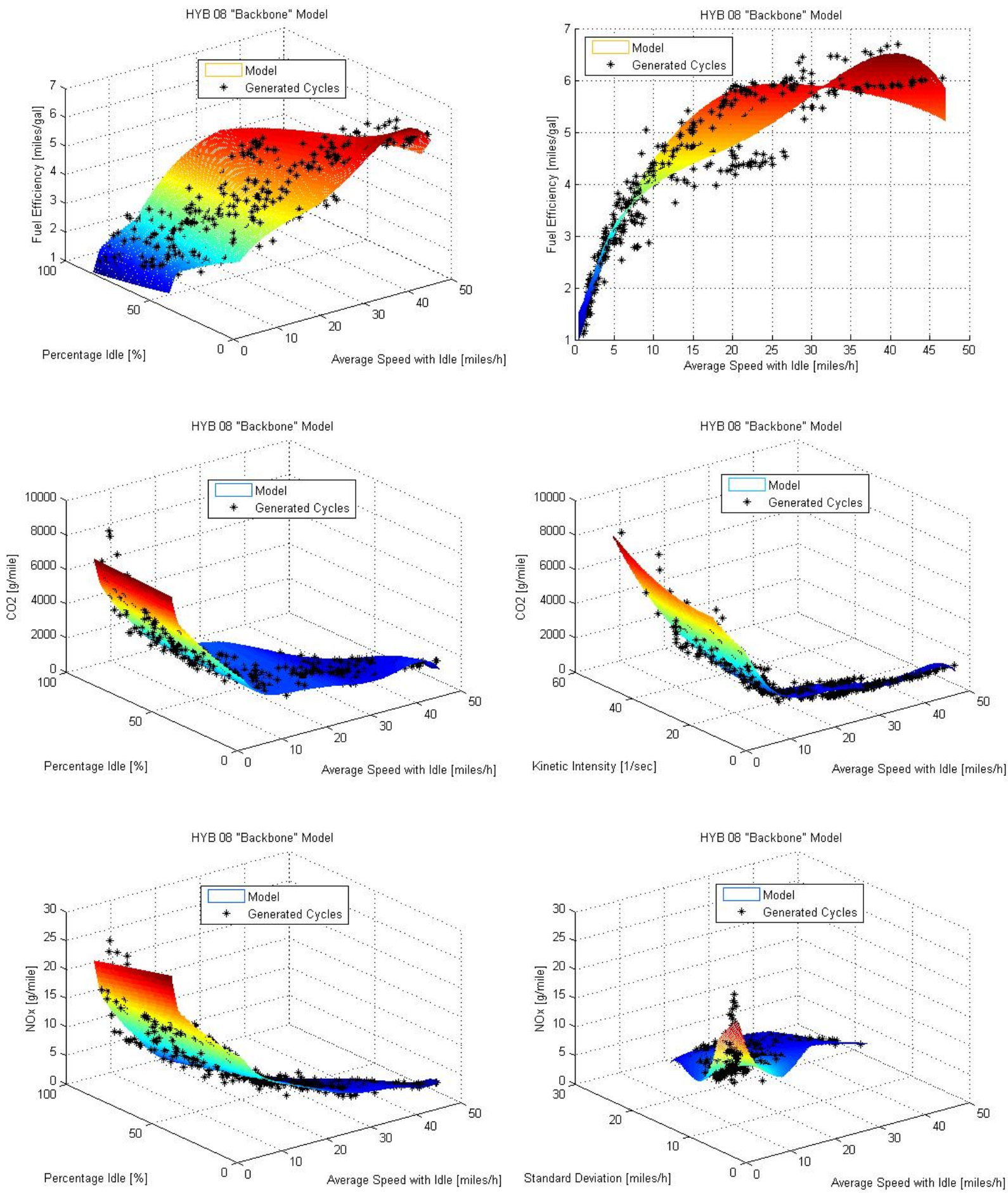

Figure 6.7 Selected Backbone Sub-Models Based on a MY 2008 Hybrid Bus 


\subsubsection{Models Based on Tested Cycles}

Besides the models presented above, models for the two buses which did not use the generated cycle points are discussed in this section. In general, when the tested cycle data shows a trend or pattern, it is very effective to use the cycle generation method to expand the limited database and develop models. In some special cases when the experimental data scattered or large spikes existed, it was not helpful to use the cycle generation method since it could not improve the original dataset. The cycle generation method can be applied to expand the size of dataset but it is not supposed to change the features of the dataset.

For example, Figure 6.8 shows the NOx emissions from the MY 2008 CNG bus. The data points include the reference cycle data which were from the emissions testing for the bus, and the newly generated virtual cycle data based on the reference cycles. Both reference and generated datasets scatter. The main reason could be attributed to the high NOx values from the several reference cycles. Especially, the COMM cycle exhibits the highest value of $3.425 \mathrm{~g} / \mathrm{mile} \mathrm{NOx}$ and it has a cruise speed over $50 \mathrm{mph}$.

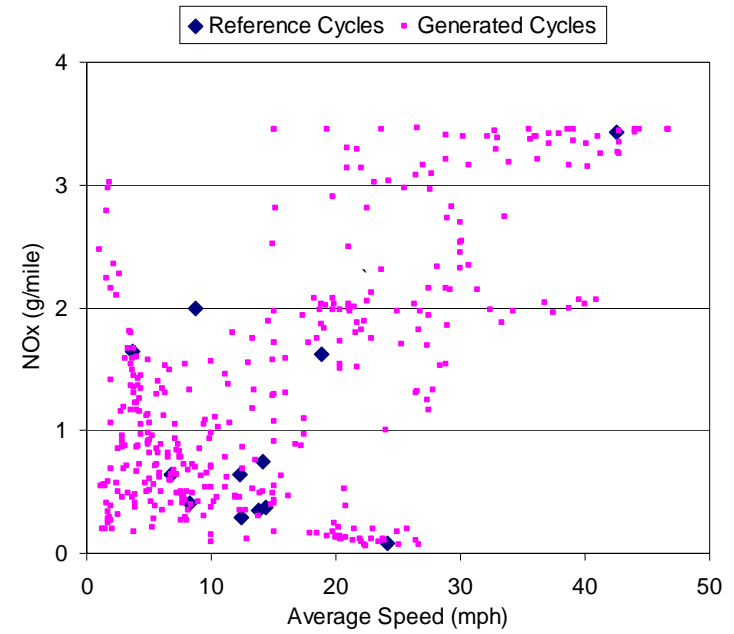

(a) With COMM cycle

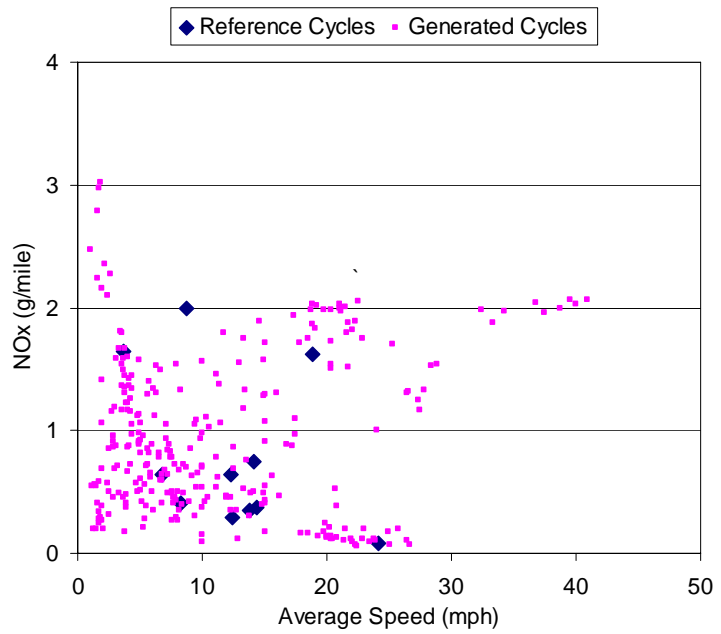

(b) Without COMM cycle

Figure 6.8 NOx Emissions from a MY 2008 CNG Bus

Figure 6.8 (a) and (b) show the difference between cycle generation with and without the COMM cycle. The NOx emission points on the both figures are scattered, indicating that the COMM cycle is not the only source of variability. Further investigation found multiple cycles had micro-trips with significantly higher NOx emissions data, such as NY-COMP, OCTA, and UDDS driving cycles. When the micro-trips from these cycles were selected during the cycle 
generation process and repeated in the same generated cycle, they would dramatically increase the distance-specific emission values of the cycle.

Figure 6.9 (a) and (b) show the examples from the COMM and OCTA cycles, respectively. Both cycles demonstrate that the high NOx emission rates exist and the micro-trips including these high rates adversely affect cycle generation. The NOx emissions reduction from this stoichiometric CNG bus was dependent on the efficiency of the three-way catalytic converter. The three-way catalytic converter operates efficiently in a narrow band near the stoichiometric point [99]. An air-fuel ratio that is too lean or too rich adversely affects the performance of the catalytic converter. Figure 6.9 (a) shows that the high NOx emissions occurred at the cruise speed.

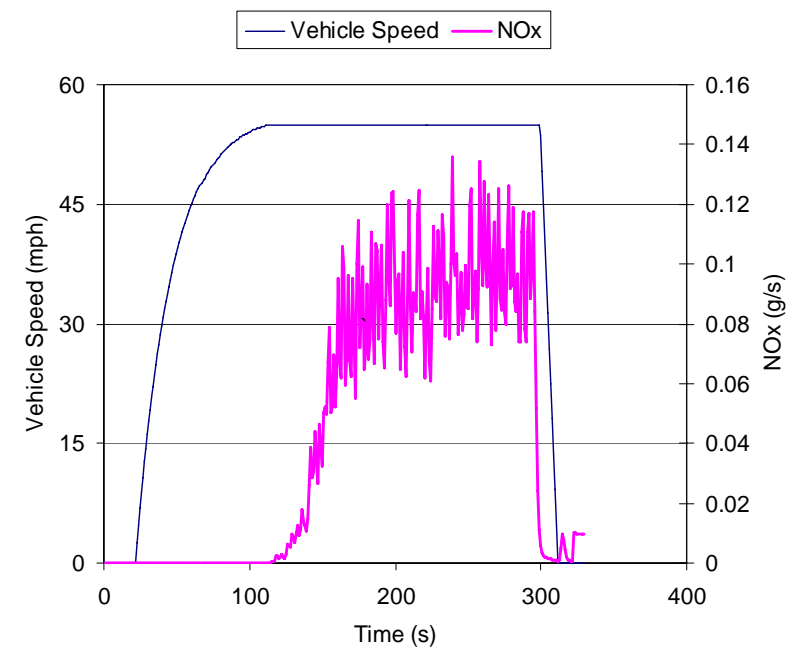

(a) COMM cycle

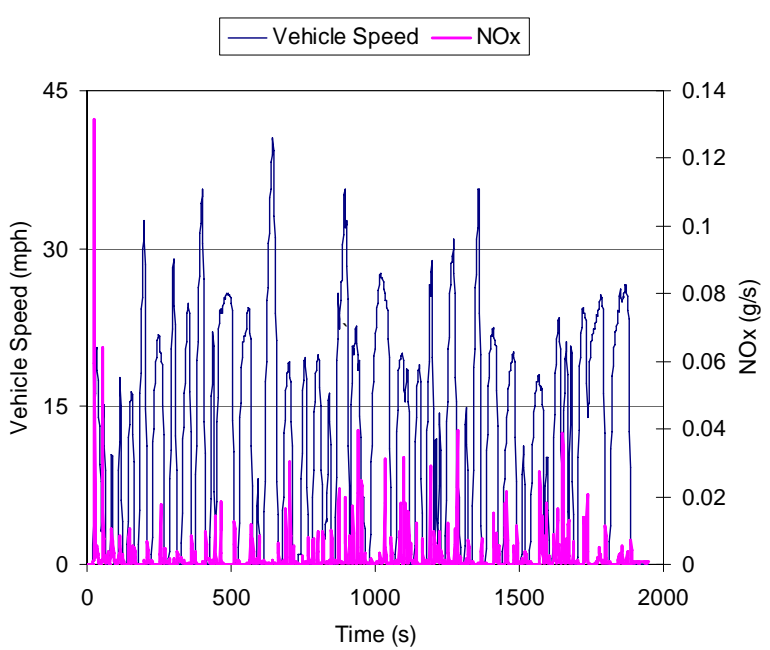

(b) OCTA cycle

Figure 6.9 NOx Emissions from a MY 2008 CNG Bus Tested on COMM and OCTA Cycles

Similar situations with high emissions in specific cycles were found for the hybrid bus as well. Figure 6.10 (a) and (b) show the $\mathrm{CO}$ emissions from the CBD and Beeline cycles, respectively. Both figures show spikes. Since this hybrid bus was equipped with DPF and DOC, the CO, HC and PM emissions were profoundly reduced. The figures show that most of time the $\mathrm{CO}$ rates were near zero except for several spikes. The CO emissions were known to be very sensitive to the transient features of vehicle operating conditions. Thus, it was easier for CO to generate spike or pulse signals. When these signals were selected and incorporated into the new generated cycle, they would significantly affect the total emissions value of the cycle. Figure 
6.11 shows the $\mathrm{CO}$ and $\mathrm{HC}$ emissions from the cycle generation for MY 2008 hybrid bus. At the lower average speed $(<10 \mathrm{mph})$, generated cycle points show high $\mathrm{CO}$ and low $\mathrm{HC}$ values.

Additionally, for cases like CO and HC emissions, the moving average filter based on 11 points was applied to smooth the data series before using the test data for modeling. The filter smoothed the second-by-second emissions data, but it could not change the pattern as shown in Figure 6.11. It was evident that those spikes were not noise thus they could not to be filtered out by the smoothing process. As a result, the quality of integrated emission values from the generated cycle would not be improved when compared to the data without smoothing.

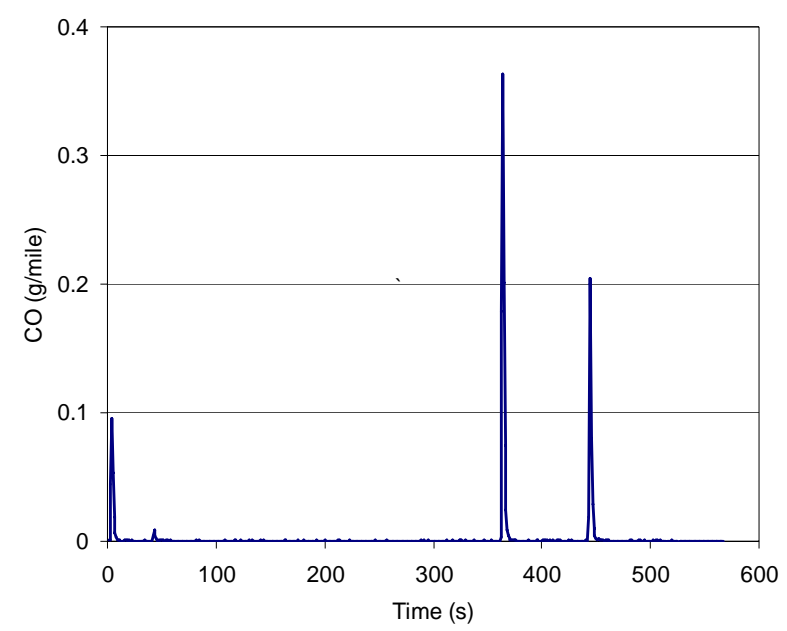

(a) CBD cycle

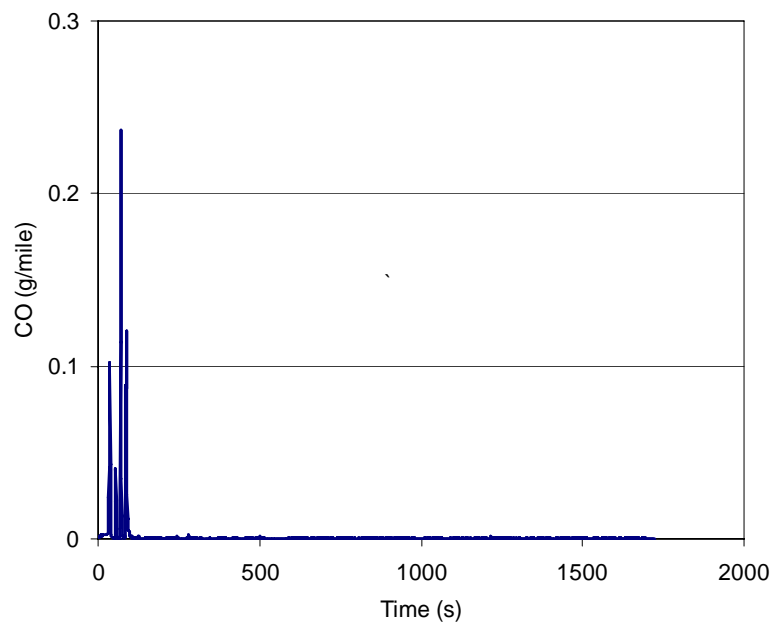

(b) Beeline cycle

Figure 6.10 CO Emissions from a MY 2008 Hybrid Bus Tested on CBD and Beeline Cycles
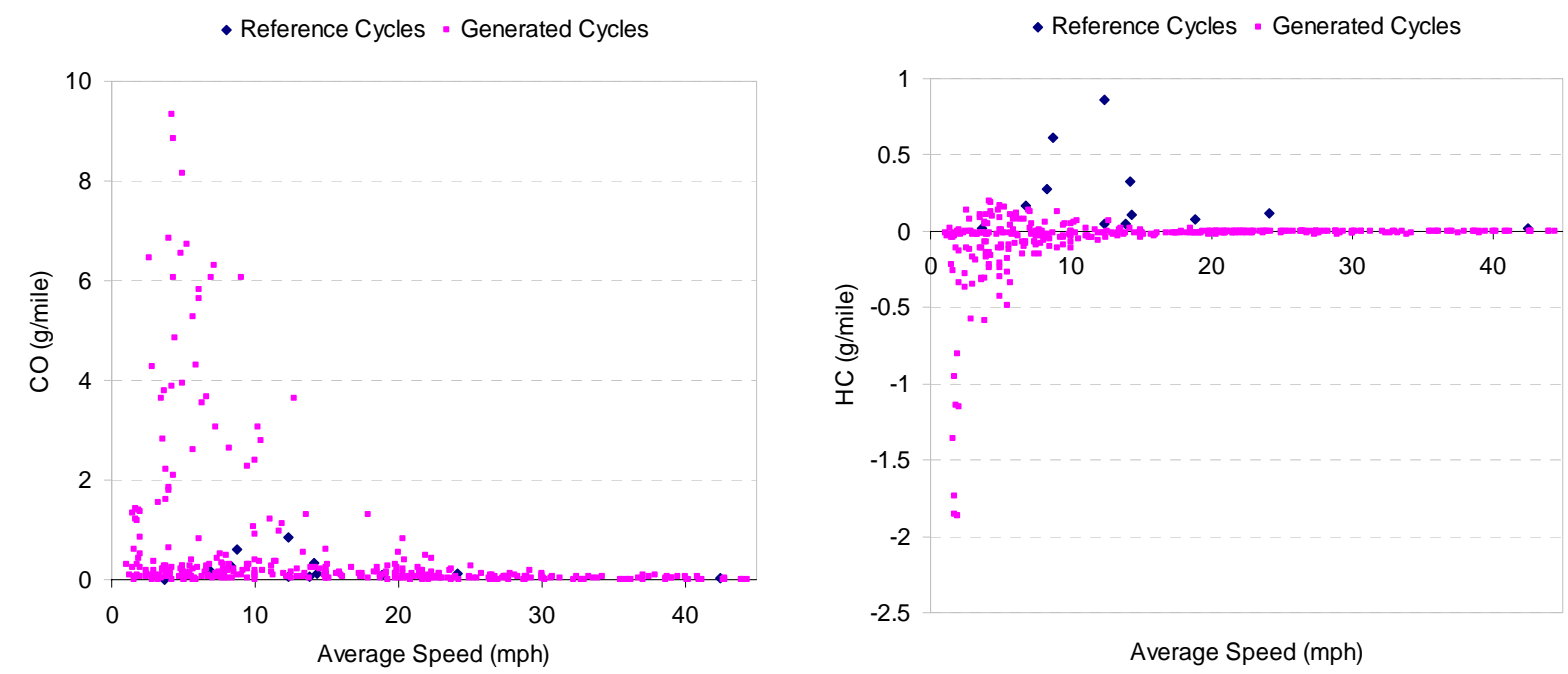

Figure 6.11 CO and HC Emissions of Tested and Generated Cycles from a MY 2008 Hybrid Bus 
After thoroughly investigating the continuous emissions data, it was found that multiple test cycles exhibited spikes for NOx and NMHC emissions from the MY 2008 CNG bus as well as $\mathrm{CO}$ and $\mathrm{HC}$ emissions from the hybrid bus. The limited number of reference cycle points was applied to develop models for these emission species as well as for the PM emissions since no instantaneous PM data was available for the cycle generation.

To avoid substantial overshoots resulted from overfitting, lower order (below three) polynomials were selected to fit these emission models. The selected sub-models are shown as Figure 6.12 and Figure 6.13. Figure 6.12 shows the sub-models of NOx and PM emissions for the MY 2008 CNG bus and Figure 6.13 shows the sub-models of CO and PM emissions for the MY 2008 hybrid bus. Compared to those models based on tested and generated cycles as discussed in the previous section, these surfaces look flatter and smoother because of the lower order of polynomials used.
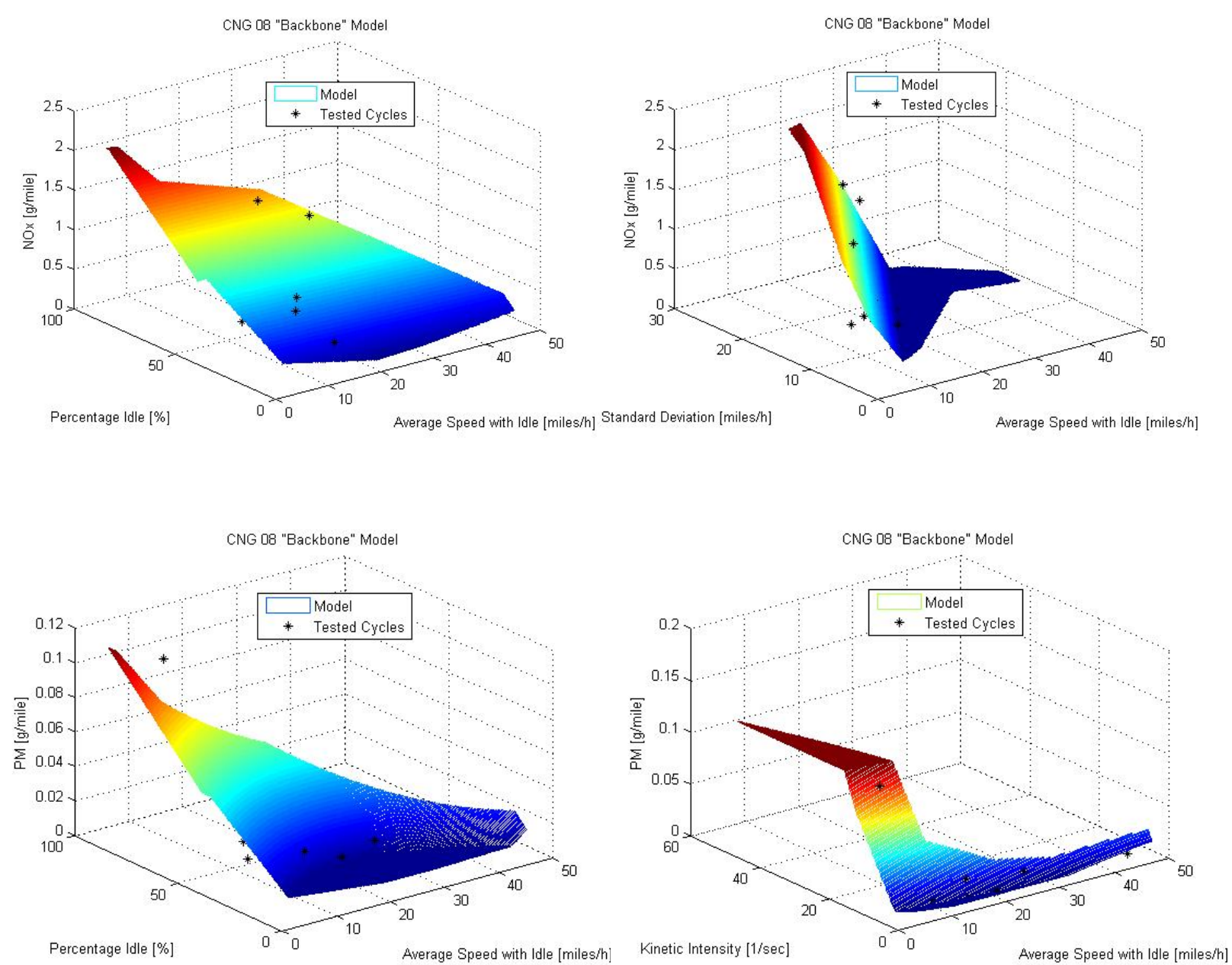

Figure 6.12 Selected NOx and PM Sub-Models of a MY 2008 CNG Bus 

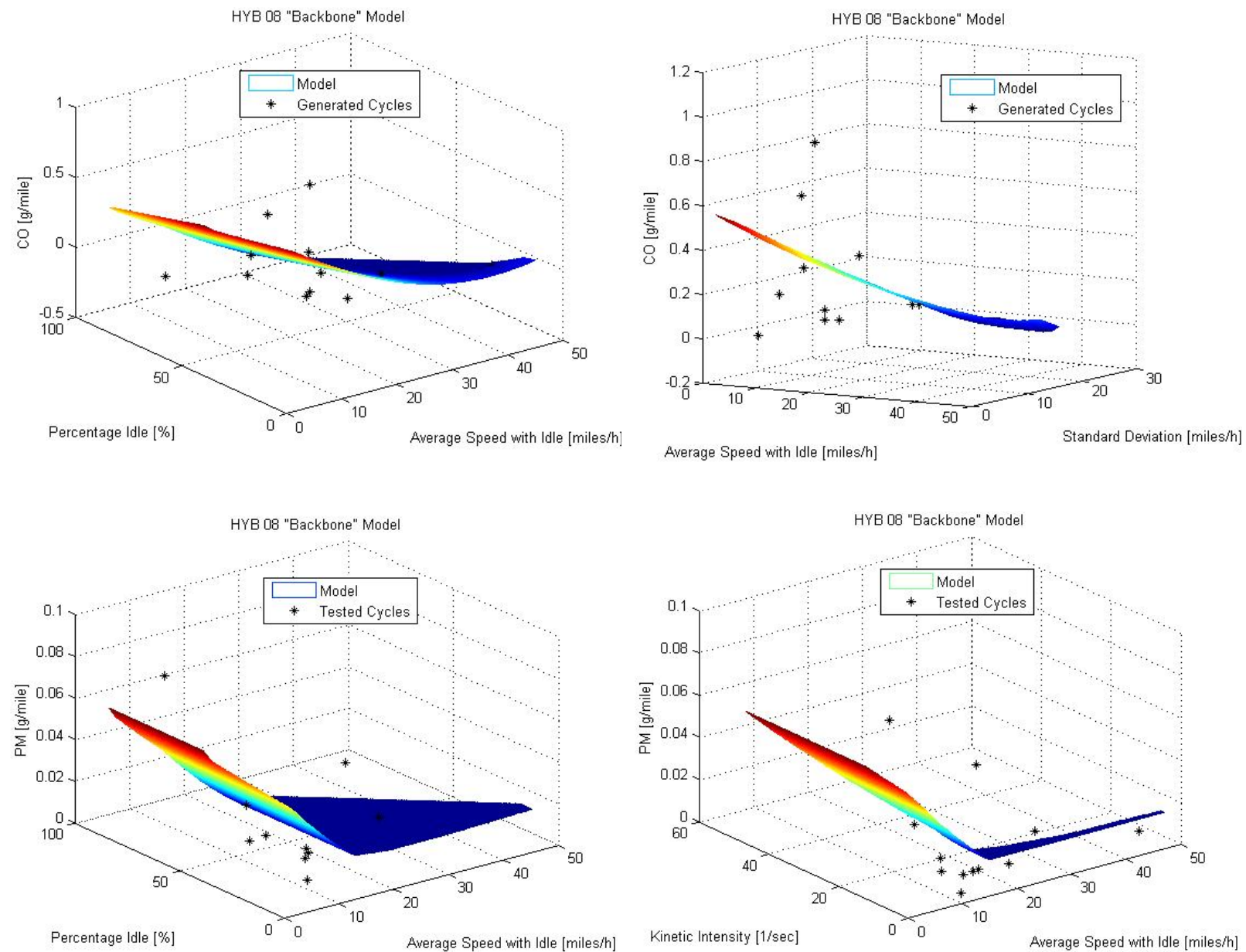

Figure 6.13 Selected CO and PM Sub-Models of a MY 2008 Hybrid Bus

Additionally, this CNG bus had integrated NMHC emission values all below zero from the 12 standard cycles. As a result, the model outputs were set as zero values. The HC emission data from the MY 2008 hybrid bus were scattered and were at low levels. Consequently, the average value from tested cycles was used as the model output.

\subsubsection{Repair Algorithm}

Usually, a repair algorithm could be used when polynomial based models did not show good fits to the data. Repair algorithms were applied to some of the older vehicle IBIS models as well [78]. Generally, it included setting limits or boundaries as well as applying piecewise functions based on the polynomial models. For example, Figure 6.14 (a) shows a NOx submodel based on kinetic intensity and average speed from the MY 2008 CNG bus and it has been projected onto a plane. In this case, a piecewise function as a horizon plane was applied at the 
lowest value point of the original polynomial curve which is the average speed at $22.3 \mathrm{mph}$. From the earlier analysis, the COMM cycle appeared as an outlier and thus was removed in this case. The NOx emissions points scatter and have higher values at the lower speed. A repaired curve shows a better fitting than a complete horizon plane in the full domain. Figure 6.14 (b) provides an example where limits were applied. Figure 6.15 demonstrates another example of repair algorithms applied to the PM sub-models for the two buses. At a specific point of average speed, a horizon plane was applied and repaired to the initial polynomial curve. The constant PM value of the horizon plane beyond specific points were determined by the average values of the tested cycles.

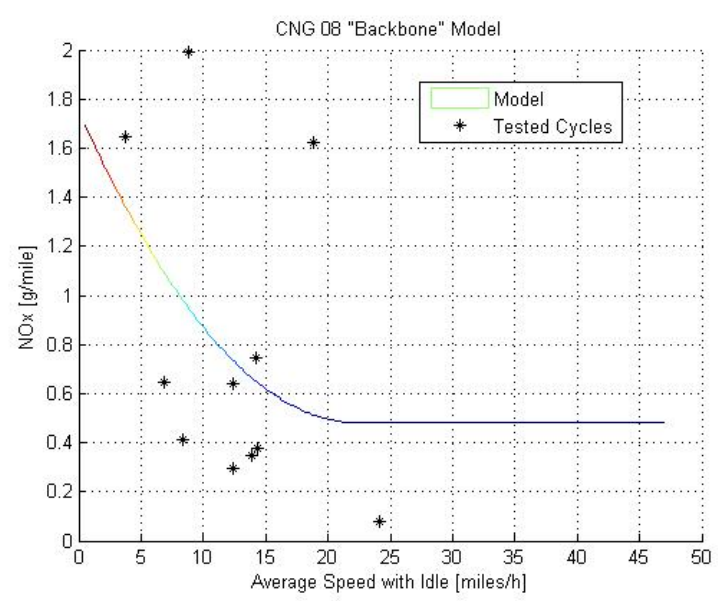

(a) Projection of Kinetic intensity sub-model

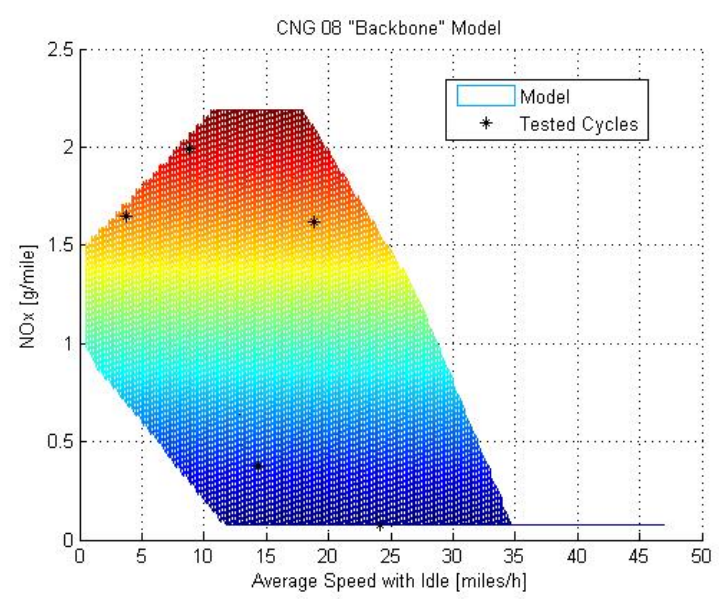

(b) Projection of standard deviation sub-model

Figure 6.14 Repair Algorithm Applied to NOx Sub-Models of a MY 2008 CNG Bus
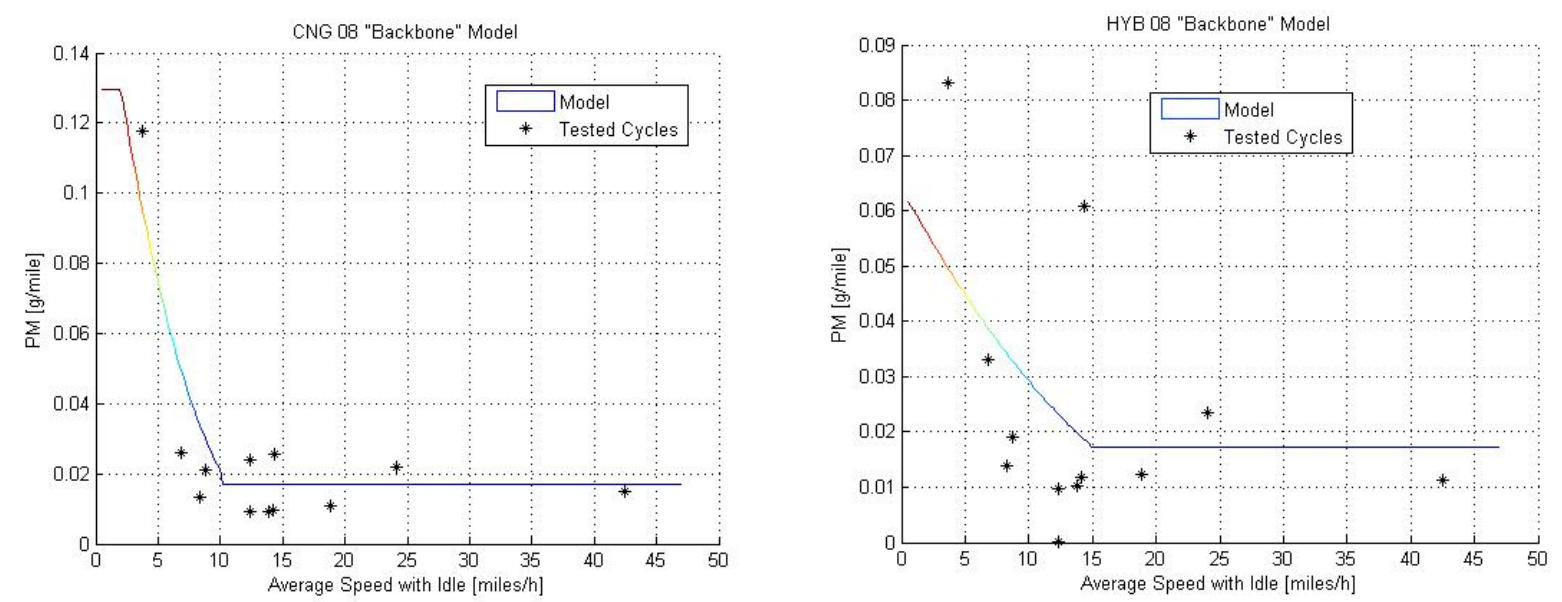

Figure 6.15 Repair Algorithm Applied to PM Sub-Models of MY 2008 CNG and Hybrid Buses 


\subsection{Model Validation}

To validate the sub-models, 34 cycle points were selected and they were not included in the calibration dataset of cycle points used to develop the models. Figure 6.16 shows validation cycle points as well as reference and generated cycle points. Most of the validation cycles were generated based on the reference cycles. Validation cycle points were selected in a way that they could be distributed on the planes spanned by the four pairs of cycle parameters of calibration cycles to cover as much area as possible.
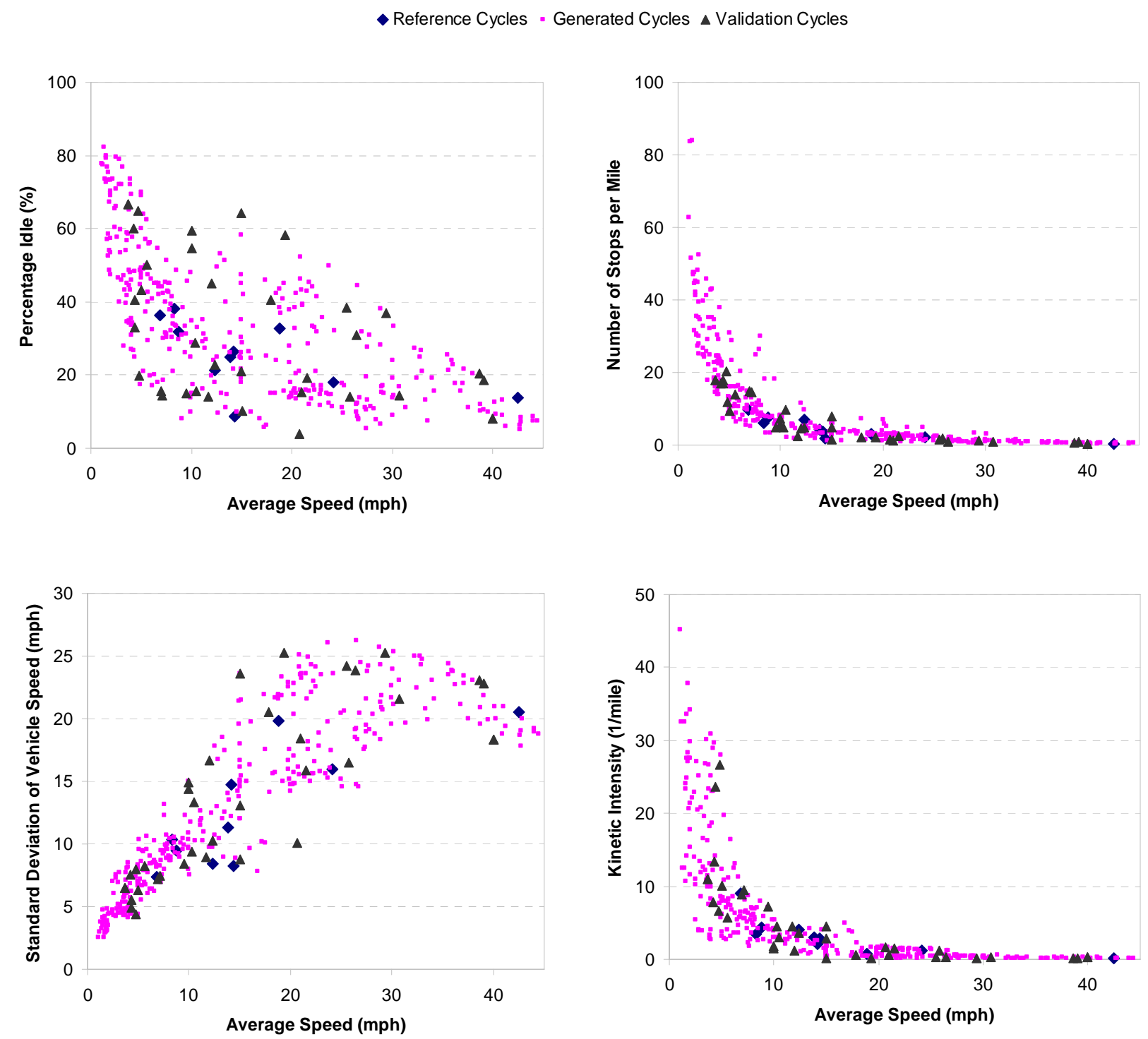

Figure 6.16 Calibration and Validation Cycle Points

In addition to the two cycle parameter based sub-models, this section provides error analysis for combined models based on three cycle parameters. 
Figure 6.17 and Figure 6.18 shows the residual plots of FE models of the CNG bus based on two and three cycle parameters, respectively. The residual was defined as the difference between the estimated value from the model and validation value from validation cycles estimated values were subtracted from the validation values. The residual plots show points are randomly distributed along with the horizontal axis.
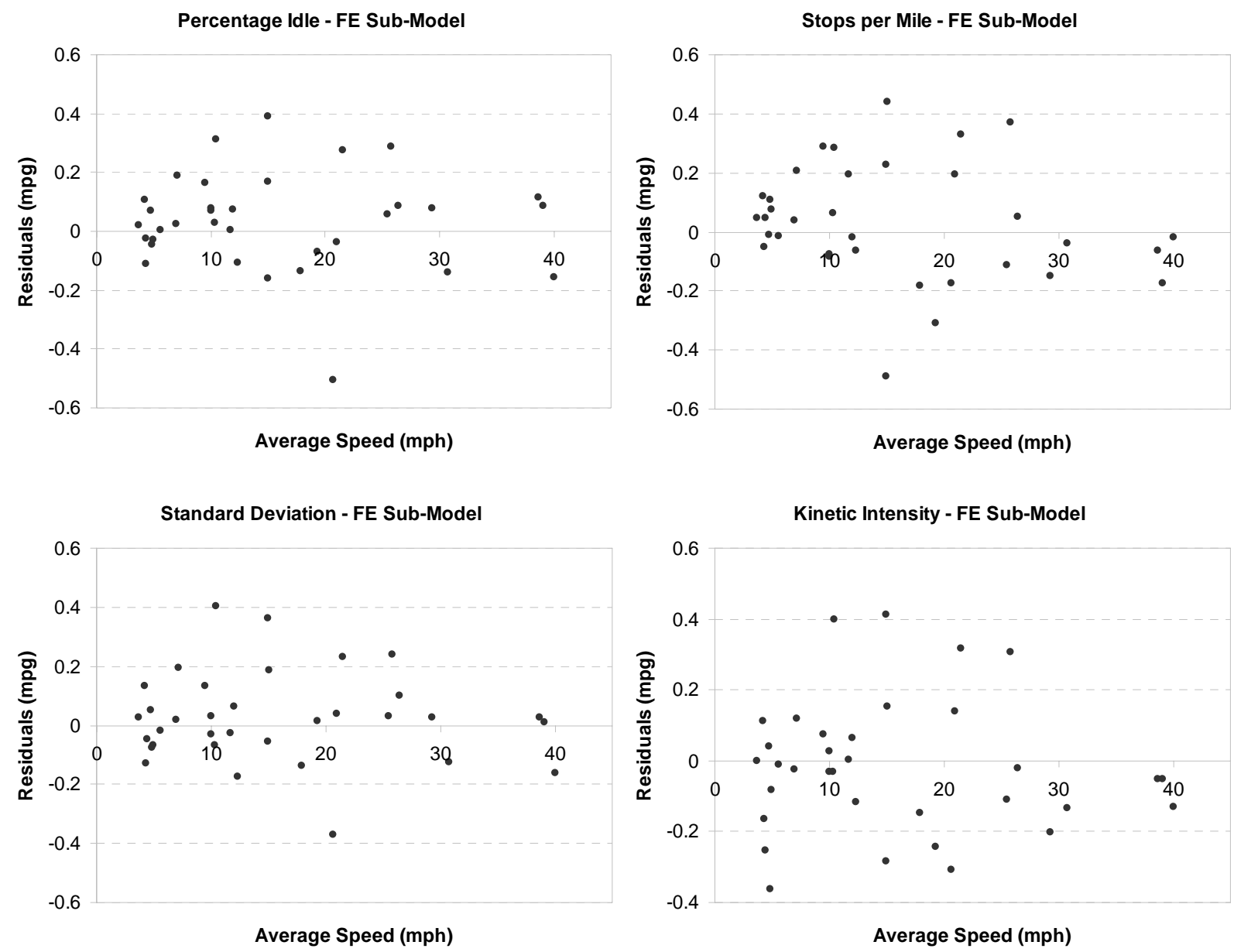

Figure 6.17 Residual Plots of Validation Cycles for the FE Sub-Models Based on Two Cycle Parameters from a MY 2008 CNG Bus 

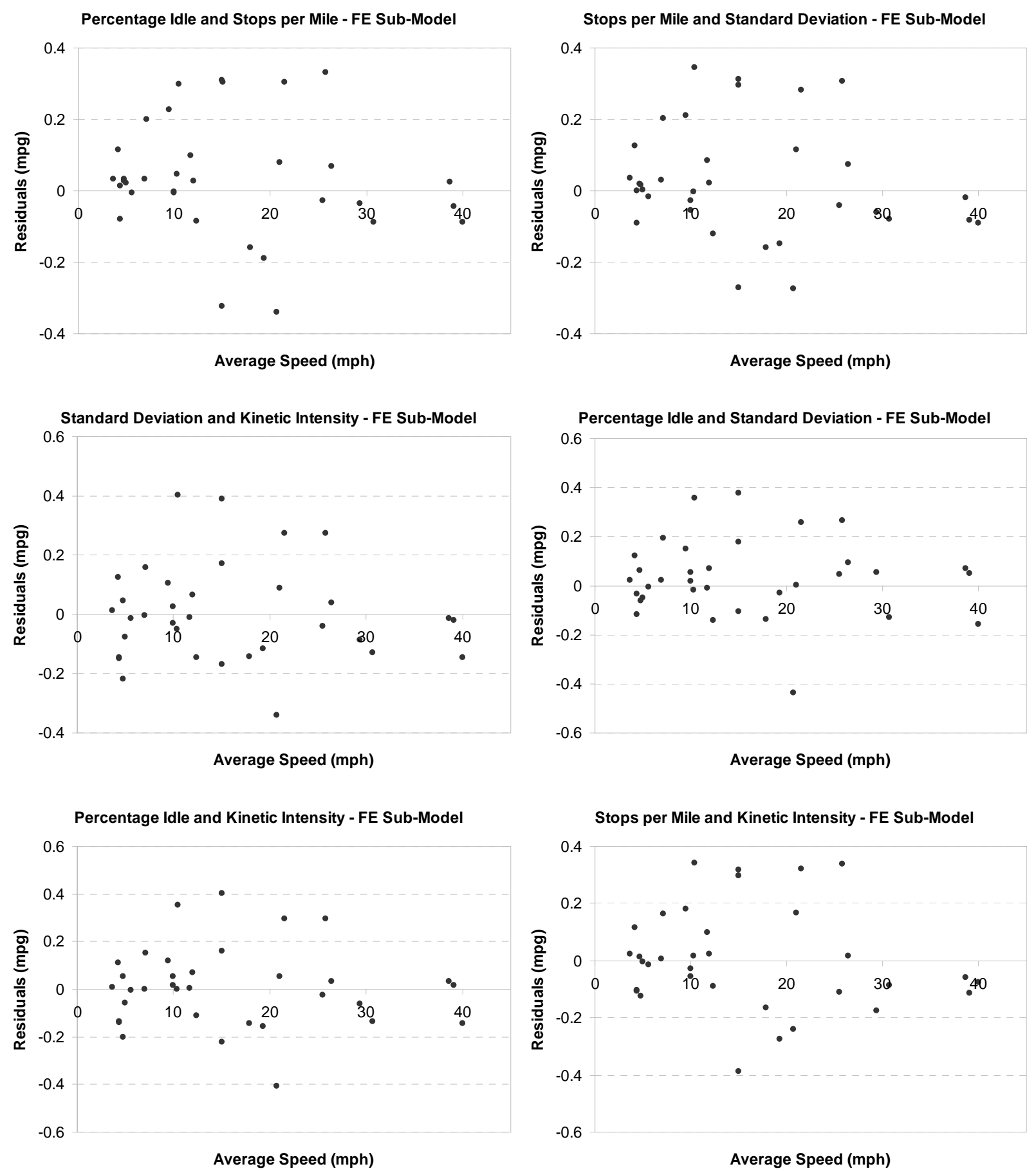

Figure 6.18 Residual Plots of Validation Cycles for the FE Sub-Models Based on Three Cycle Parameters from a MY 2008 CNG Bus

Figure 6.19 and Figure 6.20 show the average percent errors of calibration and validation cycles, respectively, for sub-models of $\mathrm{FE}, \mathrm{CO}_{2}, \mathrm{CO}$ and $\mathrm{HC}$ based on two cycle parameters for the CNG bus. These sub-models were developed based on reference and generated cycles. 
Percent errors are defined as the absolute values of residuals divided by calibration or validation cycle values. The error bars indicate the 95\% confidence level of average percent errors.

For emissions or FE sub-models based on the same two cycle parameters, their calibration errors were comparable with their validation errors, except that validation errors showed wider 95\% confident interval because the smaller number of validation cycles were used when compared with the calibration cycles. Due to the relatively stable features of $\mathrm{FE}$ and $\mathrm{CO}_{2}$ emissions with respect to vehicle operating condition, the $\mathrm{FE}$ and $\mathrm{CO}_{2}$ sub-models showed the validation errors within $7 \%$ and $9 \%$, respectively. For them, the different pairs of cycle parameters did not show the significantly different values of average percent errors. Compared with $\mathrm{FE}$ and $\mathrm{CO}_{2}$ emissions, $\mathrm{CO}$ and $\mathrm{HC}$ emissions were more sensitive to the transient features of the driving conditions thus they were more difficult to predict and their models showed greater errors. When compared between CO and HC emissions, it appears that HC emissions are more difficult to predict and show greater errors. Unlike other response variables, for HC emissions, the different pairs of cycle parameters show different average percent errors. Figure 6.20 shows that average speed with percentage idle (PI) or kinetic intensity (KI) as explanatory variables on average has smaller error (within 25\%) than average speed with stops per mile (SPM) or standard deviation of vehicle speed (STDV).

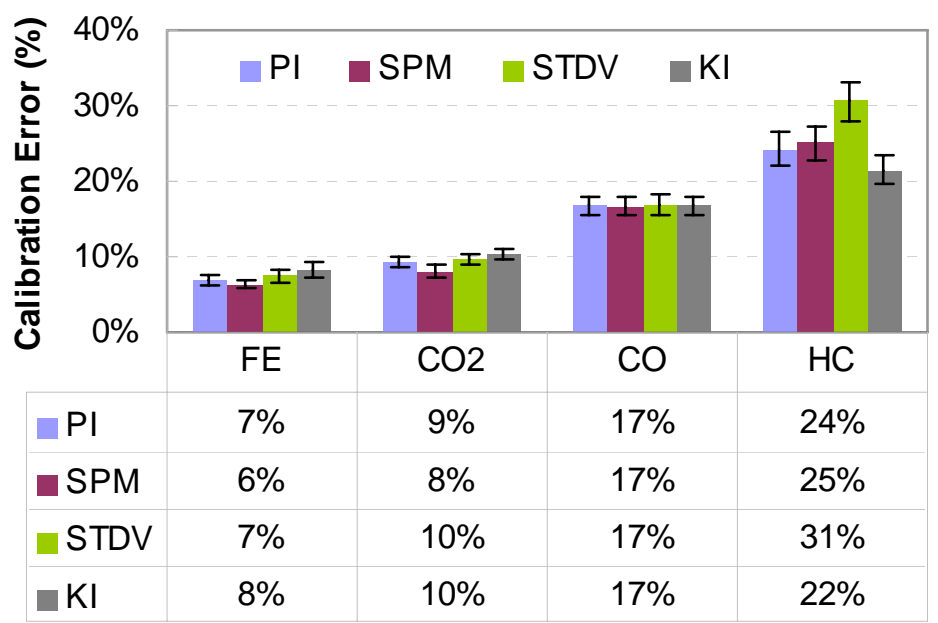

Figure 6.19 Calibration Errors of the Sub-Models Based on Two Cycle Parameters from a MY 2008 CNG Bus 


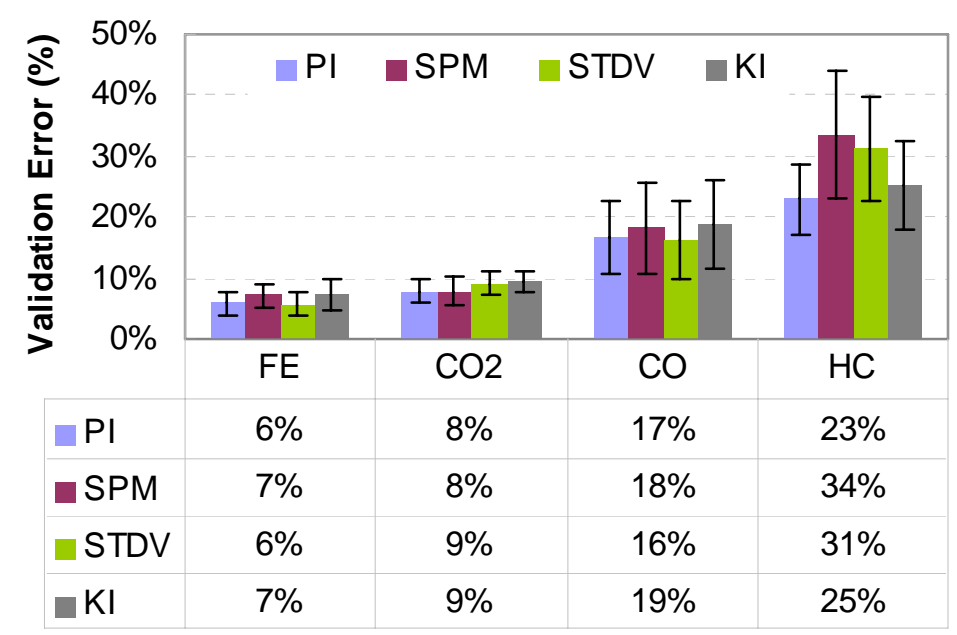

Figure 6.20 Validation Errors of the Sub-Models Based on Two Cycle Parameters from a MY 2008 CNG Bus

Figure 6.21 shows the average percent errors of validation cycles for the six combined models based on three cycle parameters of the same CNG bus. Compared to the two cycle parameters based models (Figure 6.20), for $\mathrm{FE}, \mathrm{CO}_{2}$ and $\mathrm{CO}$, an additional cycle parameter did not significantly reduce to the average percent errors of the predictive models. However, for HC emissions, adding an extra cycle parameter to the models based on average speed with SPM or STDV will reduce the average percent error to $29 \%$ or below. This is because the third cycle parameter could explain additional variance from the response variable which could not be explained by the first two cycle parameters. It might indicate, for similar CNG buses, that IBIS users could input two cycle parameters to predict $\mathrm{FE}, \mathrm{CO}_{2}$ and $\mathrm{CO}$ within acceptable accuracy level. While for HC emissions, if a third cycle parameter is available, it might be quite useful to improve the accuracy of the model. 


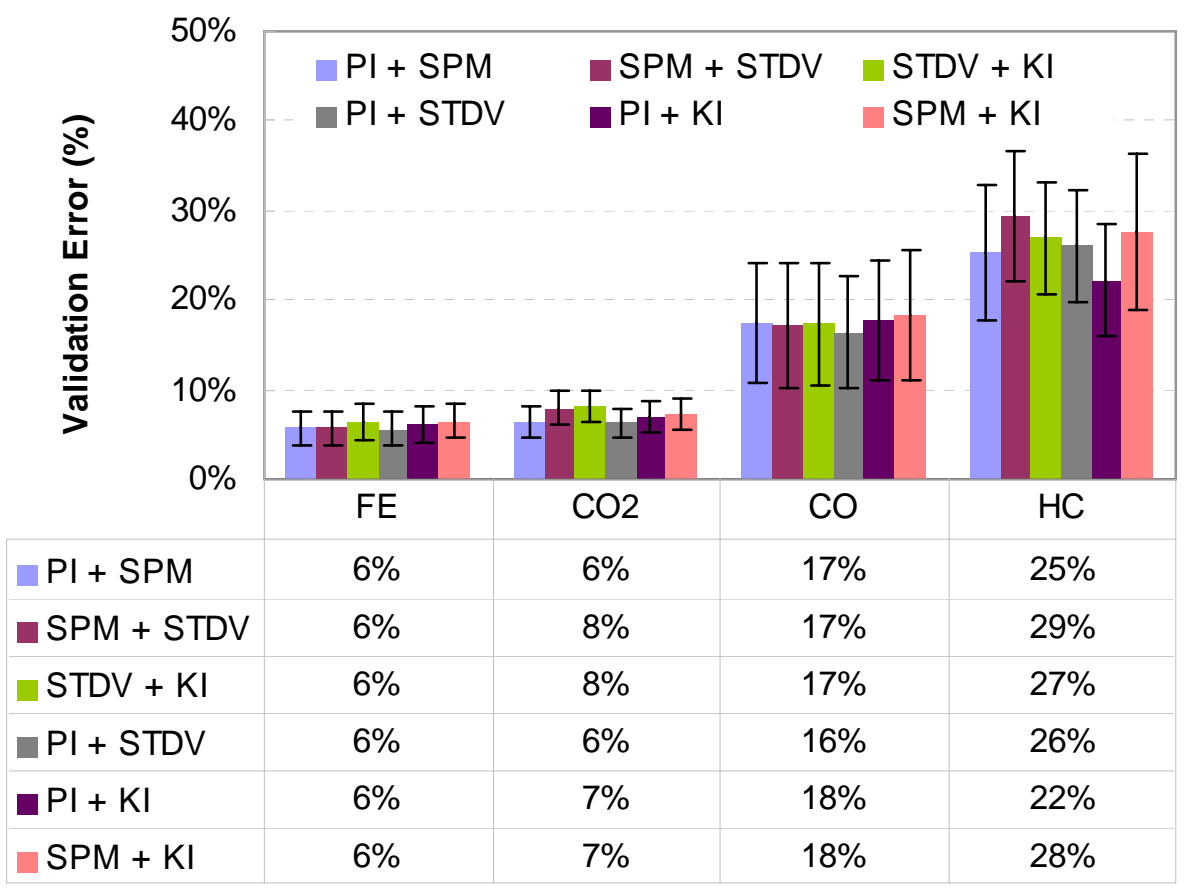

Figure 6.21 Validation Errors of the Combined Models Based on Three Cycle Parameters from a MY 2008 CNG Bus

Similar to the above analysis for the CNG bus, Figure 6.22 - Figure 6.26 are applied to the MY 2008 hybrid bus, showing the errors from its IBIS sub-models and combined models. The same calibration and validation cycles as the CNG bus were used for the hybrid bus. The FE residual plots (Figure 6.22 and Figure 6.23) show the random pattern as discussed for the CNG bus, reinforcing the good fit of predictive models. 

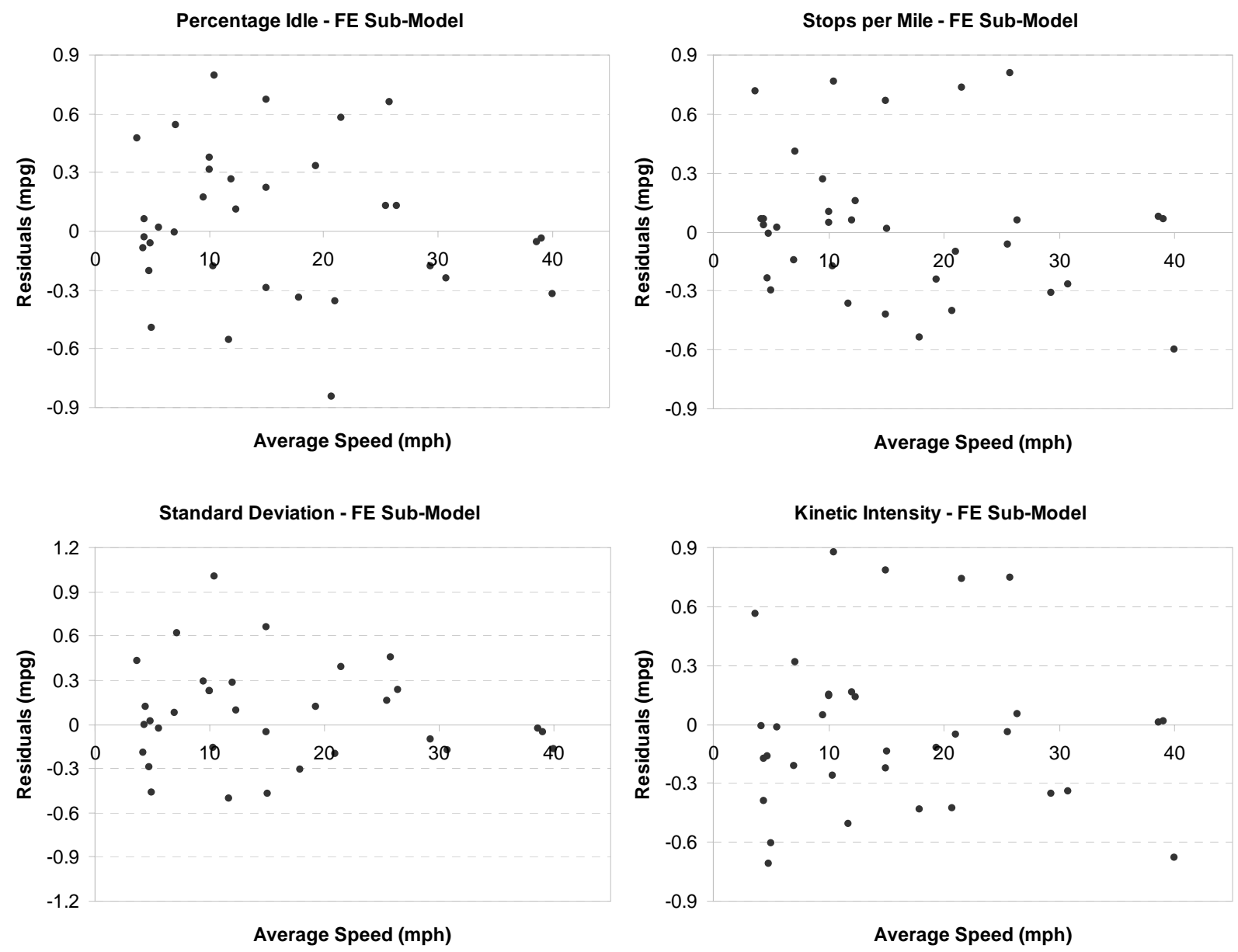

Figure 6.22 Residual Plots of Validation Cycles for the FE Sub-Models Based on Two Cycle Parameters from a MY 2008 Hybrid Bus 

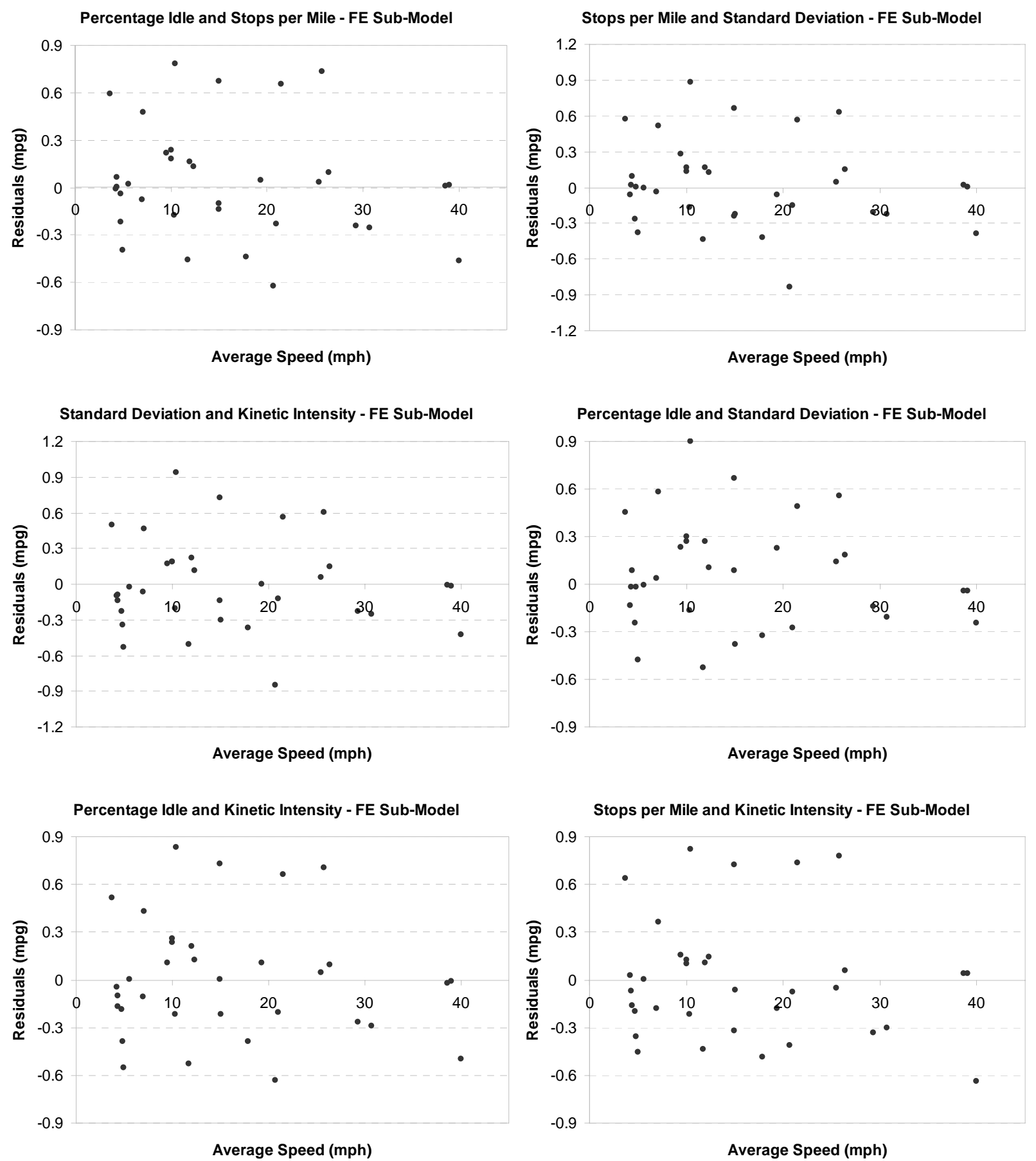

Figure 6.23 Residual Plots of Validation Cycles for the FE Combined Models Based on Three Cycle Parameters from a MY 2008 Hybrid Bus 
For the hybrid bus, the sub-models of $\mathrm{FE}, \mathrm{CO}_{2}$ and NOx were developed based on the reference and generated cycles. Figure 6.24 and Figure 6.25 show the average calibration and validation errors from the two cycle parameter sub-models, respectively. It appears that the four pairs of cycle parameters on average show comparable errors to predict FE values and all are below $8 \%$. For $\mathrm{CO}_{2}$ emissions, average speed with SPM on average predicts the FE values with smaller errors than other pairs of cycle parameters. For NOx emissions, STDV with average speed had the lowest average percent error. Figure 6.26 show the average percent errors of the combined models based on three cycle parameters. Similar to the CNG bus previously discussed, an additional cycle parameter on average could not significantly improve the models for FE and $\mathrm{CO}_{2}$ emissions. For NOx emissions, some combined models show smaller errors than the two cycle parameter sub-models.

Based on the above average validation error comparison between two cycle parameter based sub-models and three cycle parameter based combined models for MY 2008 CNG and hybrid buses, it indicated that on average additional cycle parameters beyond two would not necessarily improve the models. For $\mathrm{FE}$ and $\mathrm{CO}_{2}$ emissions, an increased number of explanatory variables (cycle parameters) on average would not significantly improve the accuracy over its individual sub-models. For other emissions such as HC emissions of the CNG bus and NOx emissions of the hybrid bus, a combined multiple variable model (more than two parameters) on average might have reduced errors based on some of its sub-models.

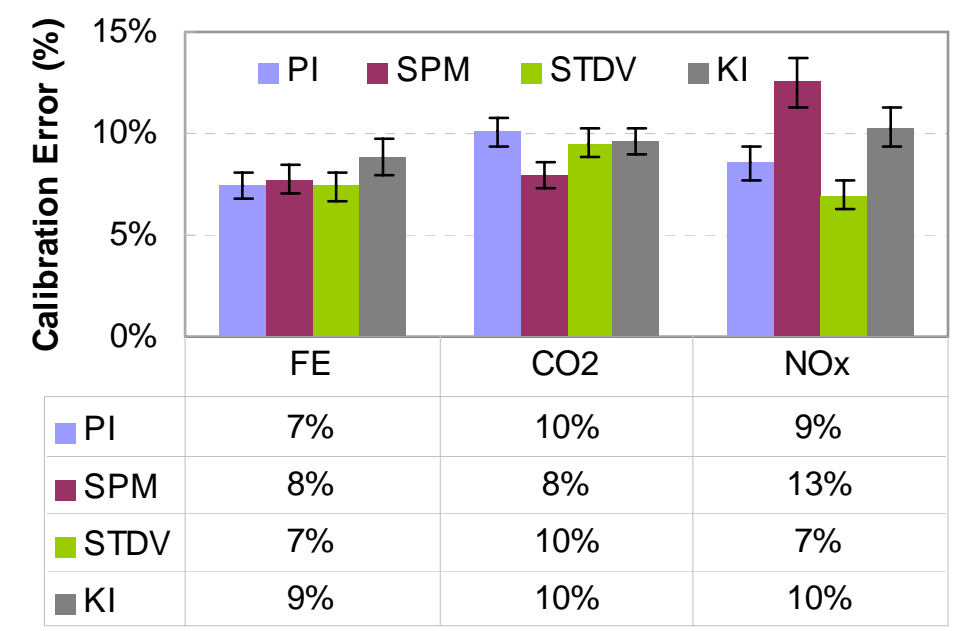

Figure 6.24 Calibration Errors of the Sub-Models Based on Two Cycle Parameters from MY 2008 Hybrid Bus 


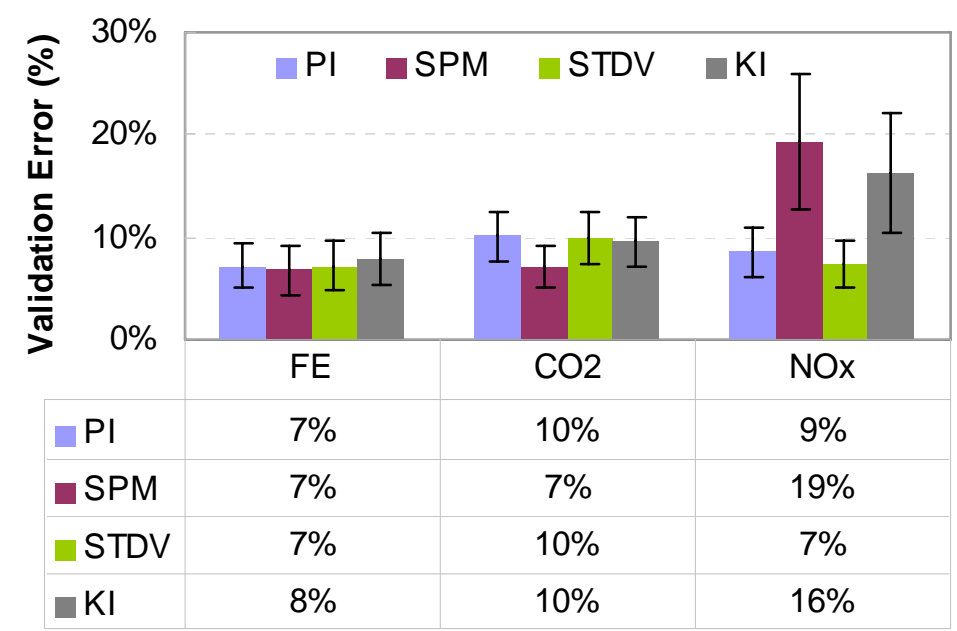

Figure 6.25 Validation Errors of the Sub-Models Based on Two Cycle Parameters from MY 2008 Hybrid Bus

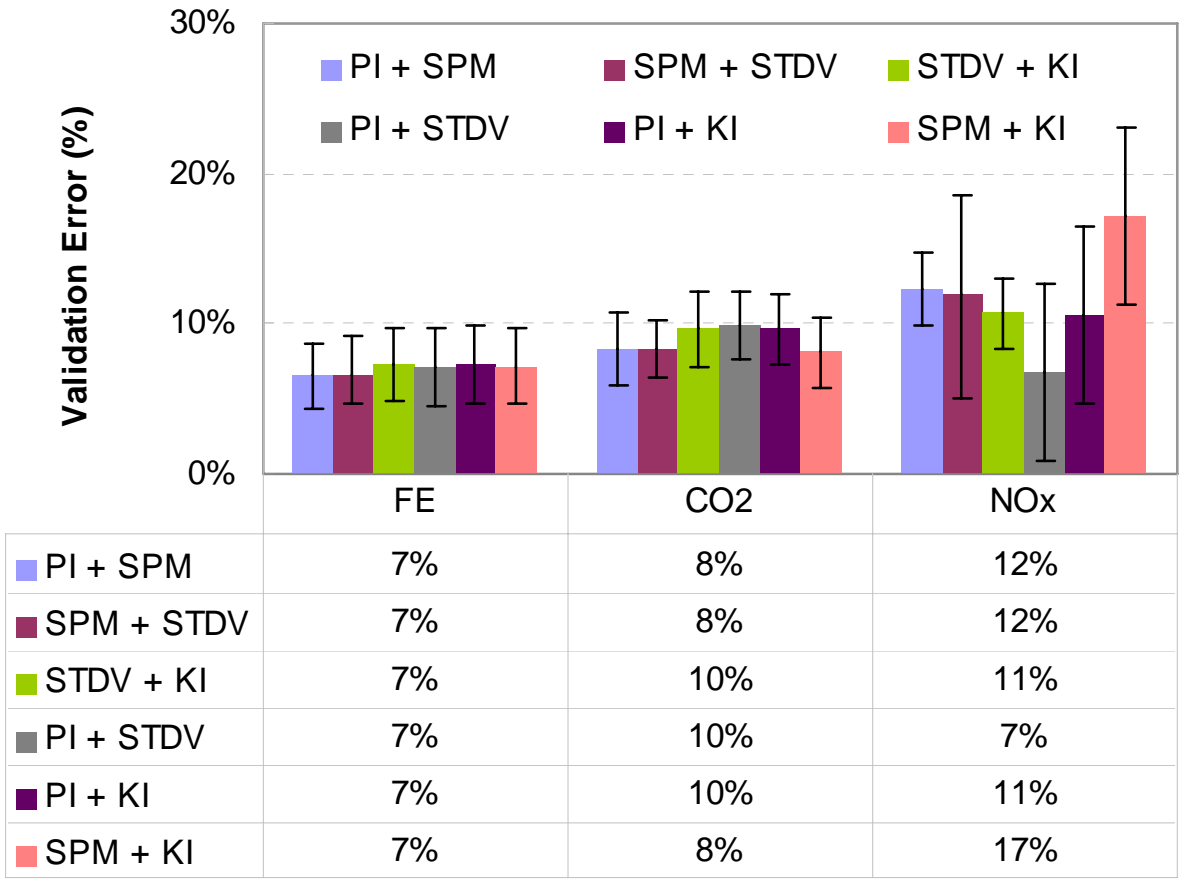

Figure 6.26 Validation Errors of the Combined Models Based on Three Cycle Parameters from MY 2008 Hybrid Bus 


\section{DEVELOPMENT OF CORRECTION FACTORS}

In addition to driving characteristics (cycle metrics), other non-cycle parameters were investigated and related correction factors were determined and applied to the backbone models to reflect their impact on emissions and fuel economy.

As mentioned in Chapter 3, multiple parameters from three categories could affect the emissions and fuel economy. In this chapter, three factors within the vehicle parameter category were investigated including vehicle weight, model year and after-treatment technologies (DPFs) for diesel vehicles.

Backbone model outputs were multiplied by correction factors if applicable, shown as Equation 3.1. As a result, correction factors were ratios and could be expressed as:

$$
\begin{array}{ll}
c_{i}(\bar{v})=\frac{Y_{i}}{Y_{\text {backbone }}} & \text { Equation } 7.1
\end{array}
$$

where $Y_{i}$ was the final response from the IBIS model including estimated emissions or fuel economy values. The $Y_{\text {backbone }}$ was the outputs of applicable backbone models or test results of selected backbone buses. The $c_{i}$ was the individual correction factor of a corresponding noncycle parameter as a function of average speed. In some cases, $c_{i}$ was assumed to be a constant and applied to the full range of the average speed.

\subsection{Vehicle Weight}

The test weight of most transit buses in this study ranged from 29,000 to 40,000 lbs. Some vehicles were tested on different weight on a chassis dynamometer to investigate how the variable loads affected the emissions and fuel economy from the transit buses. Due to the cost and effort involved in the emissions testing, a limited number of cycles was tested for those vehicles. Consequently, the microtrip based method was used to obtain more emission points to assist to assessment of the weight impact.

In this study, one diesel and one CNG bus were selected and both buses were tested on BRAUN, OCTA and PARIS cycles on three test weights: full, half and empty loads. The full load meant that a vehicle was tested with full capacity and it was equal to the curb weigh of the vehicle plus the weight of all passengers and the driver. The half load meant that a vehicle was 
tested with half capacity while empty load (unload) meant to the vehicle tested only with driver and without load (no passengers). To exclude the impact from other factors affecting emissions and fuel economy, it was intended to keep other testing parameters the same but only change the vehicle weight.

The driving characteristic from each microtrip of the above three cycles were computed and they were the five selected metrics from Chapter 4. In the meantime, for each microtrip, integrated fuel consumption and emission values except for the PM were calculated and distance-specific emission values as well as fuel economy were obtained. For PM emissions, only three test cycle values were used due to the lack of continuous PM data. In total, 107 microtrips were obtained and their average speed ranged from below $0.5 \mathrm{mph}$ to $22.7 \mathrm{mph}$. In this way, instead of three tested cycles, the weight impact on emissions and fuel economy could be investigated from a variety of average speeds.

Seven out of these microtrips were found to have average speeds below $0.5 \mathrm{mph}$ and over $60 \%$ idle associated with substantially high distance-specific emission values for both diesel and CNG buses. These microtrips were considered to the equivalent of idle and were included for the analysis.

For each bus, to investigate weight impact, emissions and fuel economy ratios between full and half load as well as ratios between full and empty load were calculated and plotted as a function of average speed of all microtrips and tested cycles.

The road load equation was studied and derived to further explore the relationship between vehicle weight and emissions and fuel economy. As a result, simplified weight correction factors were developed as a function of average speed.

\subsubsection{Diesel Bus}

The selected diesel bus was a MY 1992 40-foot diesel bus retrofitted with a MY 2003 Detroit Diesel S50 engine and diesel particulate filter (DPF). The bus was powered with ULSD1 fuel and tested with full load of 39,410 lbs, half load of 34,610 lbs and empty load of 29,810 lbs. The emissions and fuel economy values of full and empty load from the three tested cycles associated with their microtrips are shown in Figure 7.1 and the ratios are shown in Figure 7.2.

The $\mathrm{CO}_{2}$, NOx and fuel economy showed trends with respect to average speed and most of their ratios were greater than one for emissions and less than one for fuel economy. The ratios 
were calculated based on values of full load compared to those of half load and empty load, respectively. Figure 7.2 also shows that the two types of ratios are separated with individual trends line for $\mathrm{CO}_{2}$, NOx and fuel economy. In addition, it shows that greater weight difference resulted in greater ratio values for emissions while smaller ratio values for fuel economy. It indicated that for the diesel bus higher passenger load resulted in more $\mathrm{CO}_{2}$, and $\mathrm{NOx}$ emissions and more fuel consumed.

Both Figure 7.1 and Figure 7.2 show that CO, HC and PM emissions have no clear trend as a function of average speed since these emissions points scatter. Their ratio points for different weight mixed, showing that some values were larger than one while others were smaller. Thus, it was difficult to assess the impact of vehicle weight on these emissions. 

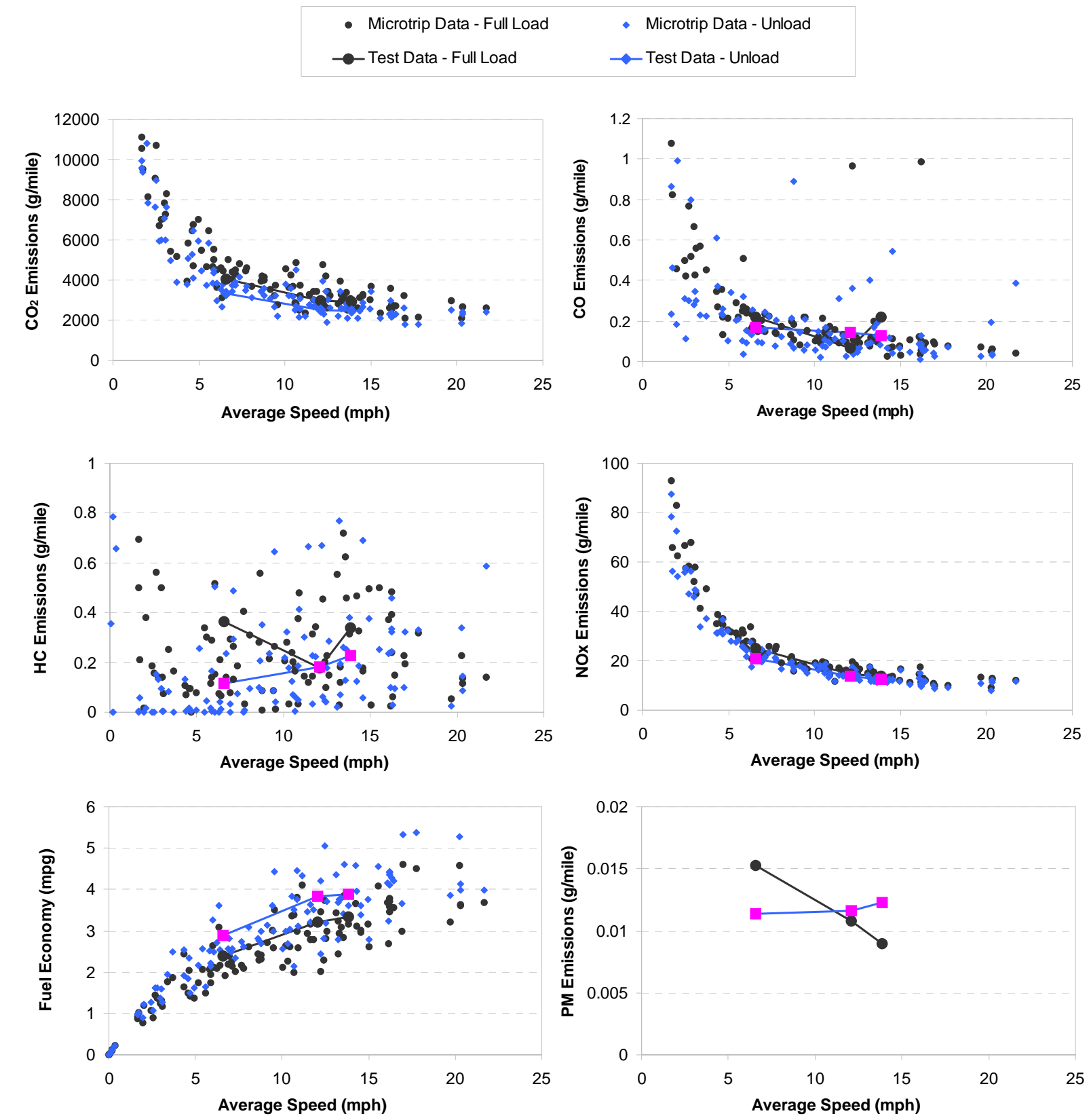

Figure 7.1 Emissions and Fuel Economy of a MY 1992 Diesel Bus Retrofitted with a MY 2003 Detroit Diesel Engine Tested at Different Weights 


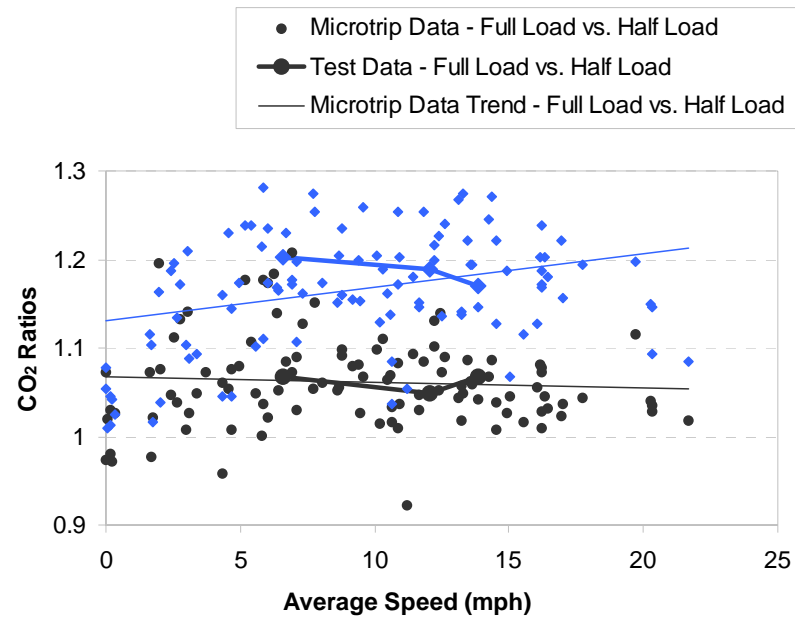

Microtrip Data - Full Load vs. Unload
— Test Data - Full Load vs. Unload
— Microtrip Data Trend - Full Load vs. Unload
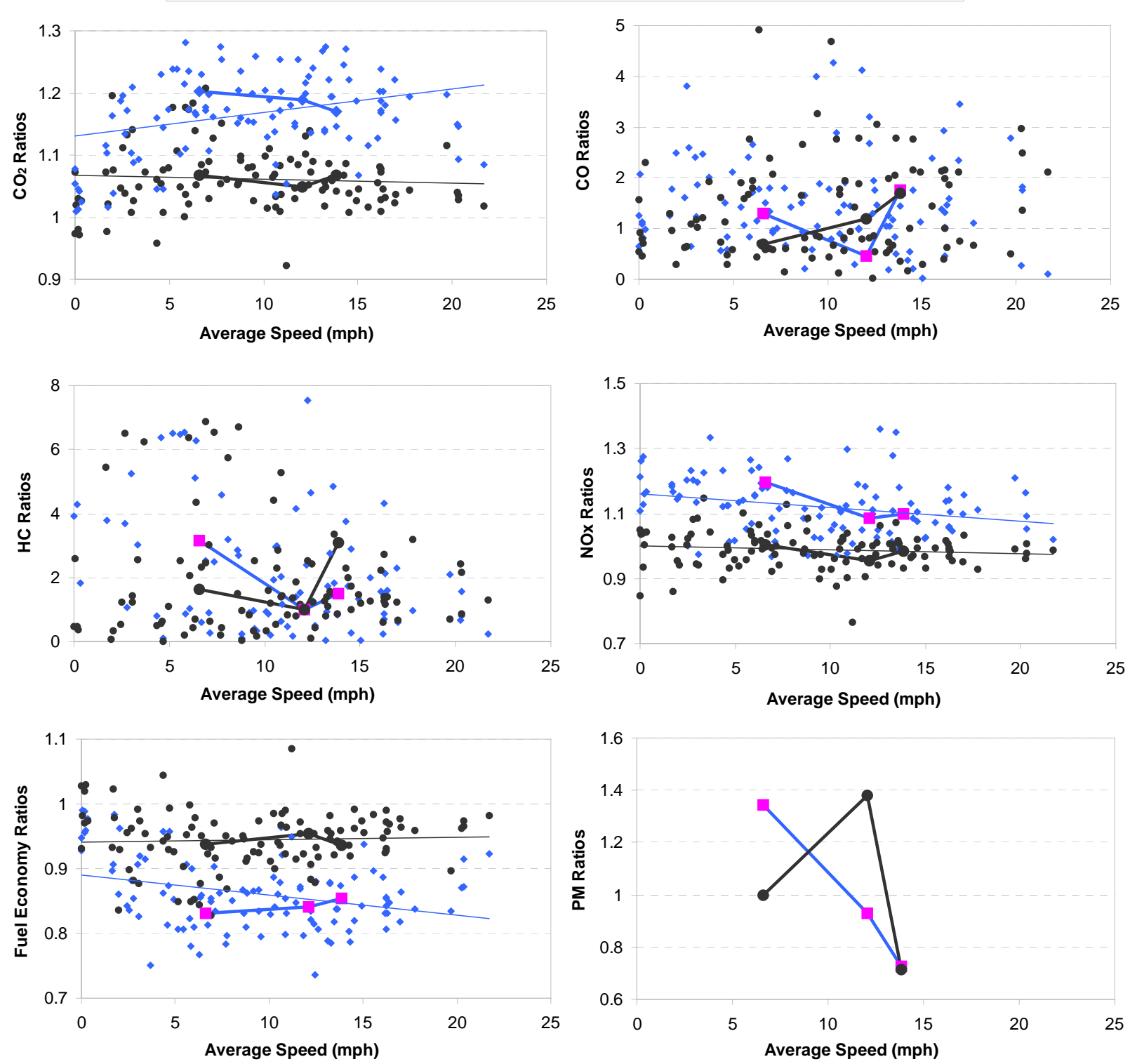

Figure 7.2 The Ratios of Emissions and Fuel Economy of a MY 1992 Diesel Bus Retrofitted with a MY 2003 Detroit Diesel Engine Tested at Different Weights

Figure 7.3 shows the average ratios based on all microtrips and tested cycles at different weight and error bars indicate standard deviation of the ratios. It reinforces that vehicle weight affects emissions and fuel economy. CO and HC show substantially higher standard deviation than others, which is consistent with their scattered pattern. For $\mathrm{CO}_{2}$ and fuel economy, Figure 7.3 shows that on average a $14 \%$ increase in weight leads to $6 \%$ higher $\mathrm{CO}_{2}$ emissions and $6 \%$ lower fuel economy. A $32 \%$ increase in weight leaded to $17 \%$ higher $\mathrm{CO}_{2}$ emissions and $14 \%$ 
lower fuel economy. As a result, an A\% weight increase could roughly increase $\mathrm{CO}_{2}$ emissions and reduce fuel economy by $0.5 \mathrm{~A} \%$. For NOx emissions, a $14 \%$ increase in weight on average had little effect on the emissions while a 32\% increase in weight resulted in $12 \%$ higher NOx emissions. The small standard deviation of the ratios from $\mathrm{CO}_{2}, \mathrm{NOx}$ and fuel economy indicated that the weight impact on them was not sensitive to average speed of the vehicle which can be observed in Figure 7.2 as well due to the flat trend lines.

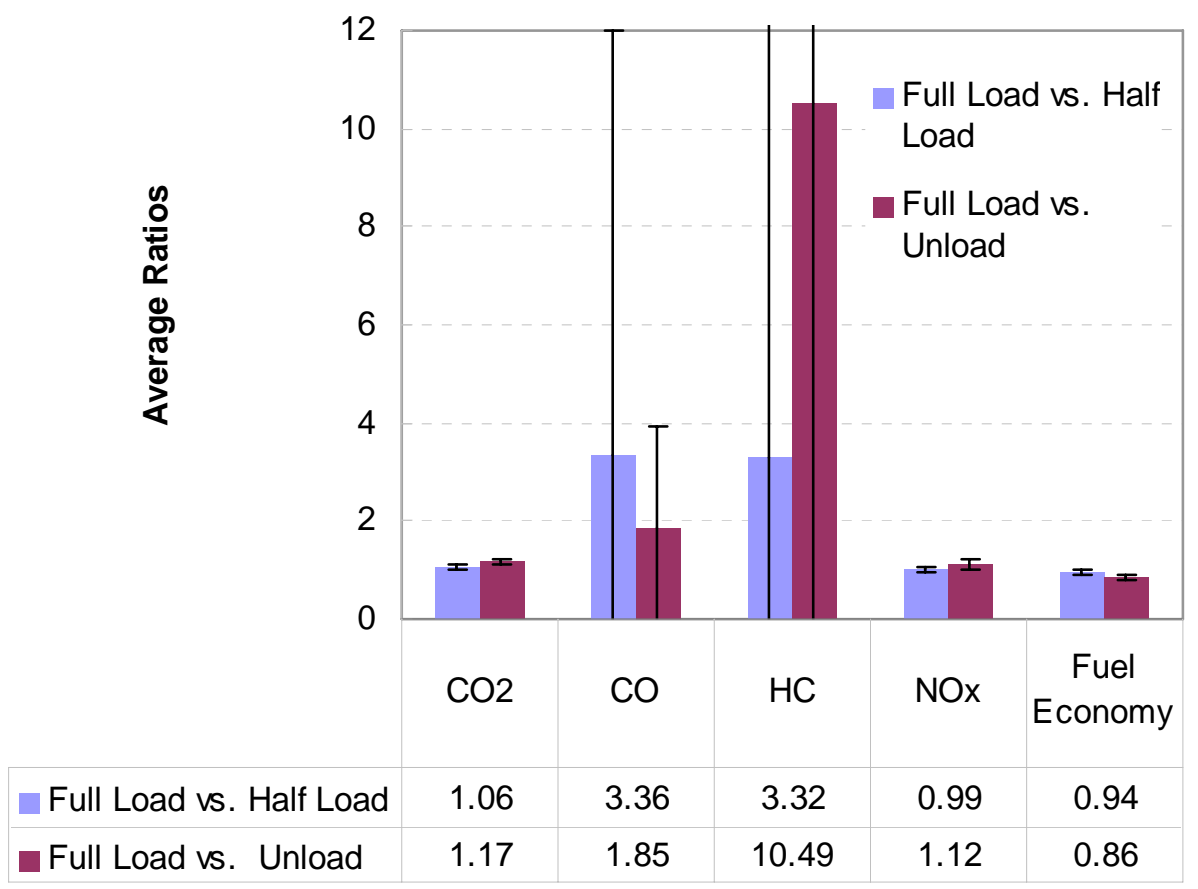

Figure 7.3 The Average Ratios of Emissions and Fuel Economy Based on Microtrip Data from a MY 1992 Diesel Bus Retrofitted with a MY 2003 Detroit Diesel Engine Tested at Different Weights

\subsubsection{CNG Bus}

The selected CNG bus was a MY 2005 40-foot CNG bus equipped with a John Deere engine and a catalytic converter. The test weight of full, half and empty load were 41,470, 36,970 and 32,470 lbs, respectively.

Figure 7.4 shows the ratios of different weight as a function of average speed of microtrips and tested cycles. Similarly, $\mathrm{CO}_{2}$, NOx and fuel economy show clear trend while $\mathrm{CO}$, HC and PM emission points scatter. Figure 7.5 shows average ratios with error bars indicating standard deviation of the ratios. For the CNG bus, a 12\% increase in weight from half to full load on average did not significantly affect the $\mathrm{CO}_{2}$ and fuel economy while a $28 \%$ increase in weight from empty to full load increased $\mathrm{CO}_{2}$ emission by $11 \%$ and reduced fuel economy by $10 \%$. The 
impact of vehicle weight on the CNG bus did not follow the same way as it did on the diesel bus. The CNG bus showed on average $4 \%$ higher HC emissions due to a $12 \%$ increase in weight from half to full load and $7 \%$ higher HC emissions due to a $28 \%$ increase in weight from empty to full load. Compared to the diesel bus, the CNG bus had significantly smaller coefficient of variance (CV) of the ratios in terms of HC emissions.

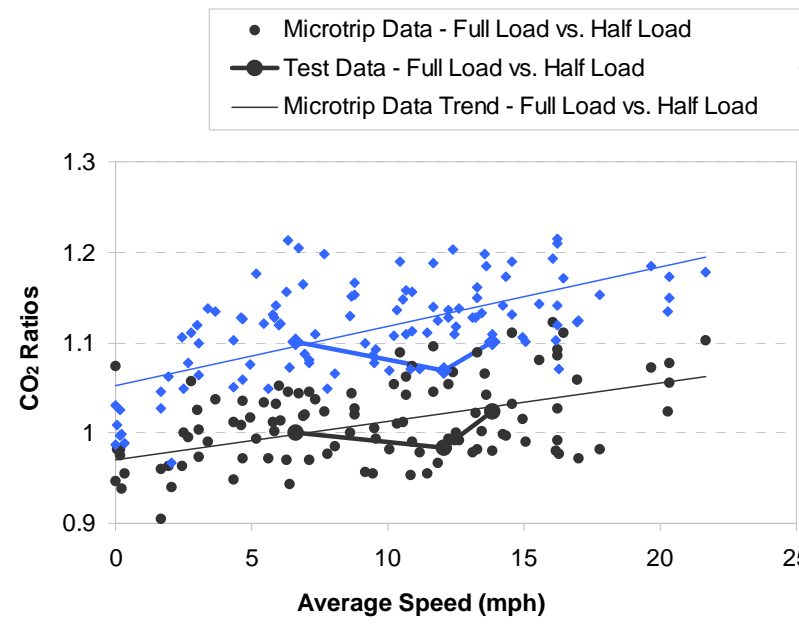

Microtrip Data - Full Load vs. Unload
$\_$Test Data - Full Load vs. Unload
— Microtrip Data Trend - Full Load vs. Unload
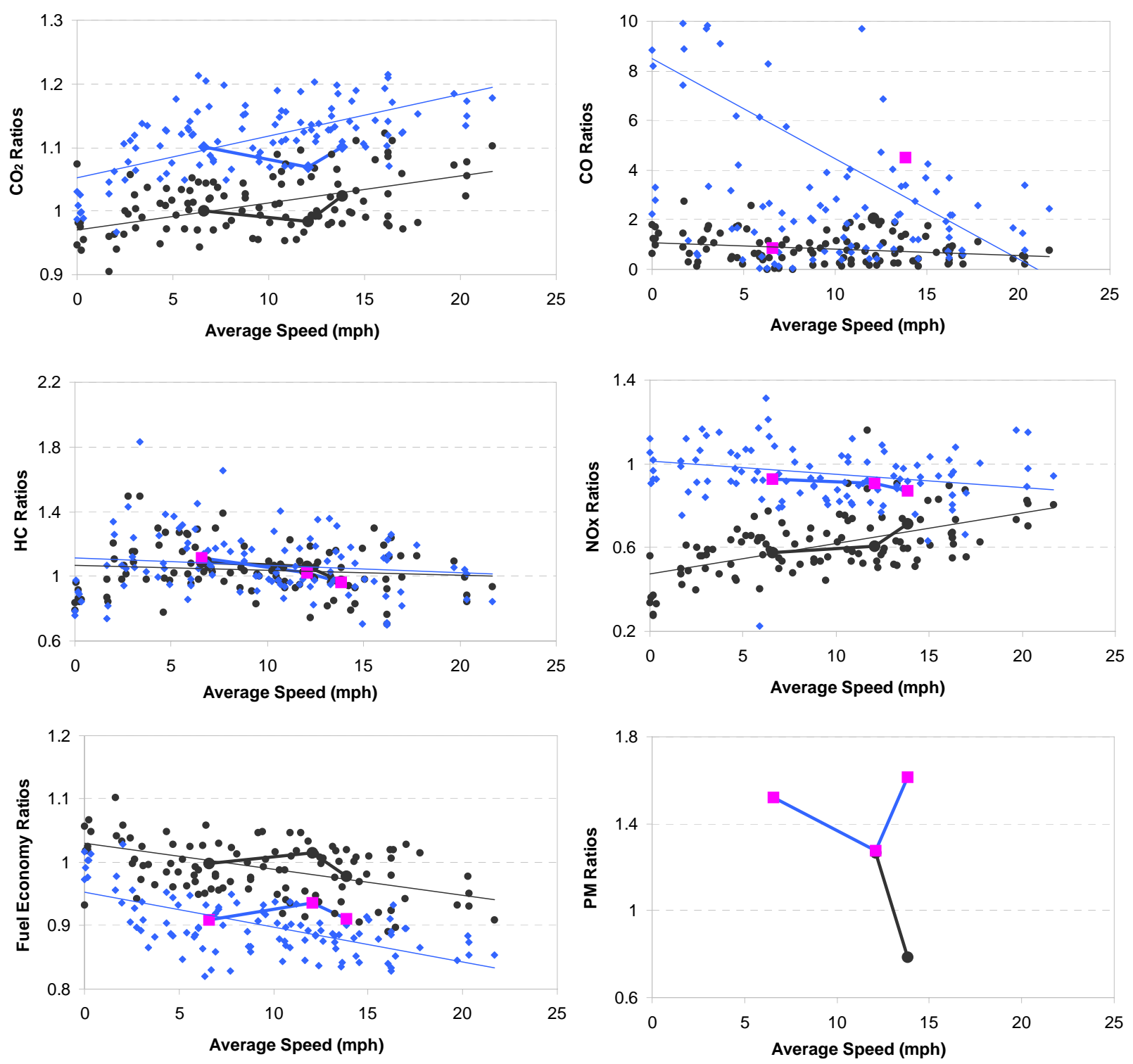

Figure 7.4 The Ratios of Emissions and Fuel Economy of a MY 2005 CNG Bus with a John Deere Engine Tested at Different Weights 
In addition, Figure 7.4 and Figure 7.5 show that NOx emissions are lower in full load than half and empty load although they present trends along the average speed. In general, heavier weight was supposed to exhibit higher NOx emissions than lighter weight. Further investigation showed on average $65 \%$ higher NOx emissions due to the $14 \%$ increase in weight from empty to half load and the ratios of NOx emissions showed 32\% CV. It was difficult to believe that $14 \%$ increase in weight could lead to $65 \%$ higher emissions. Due to the limited dataset, there might have relatively large experimental errors from NOx emissions.

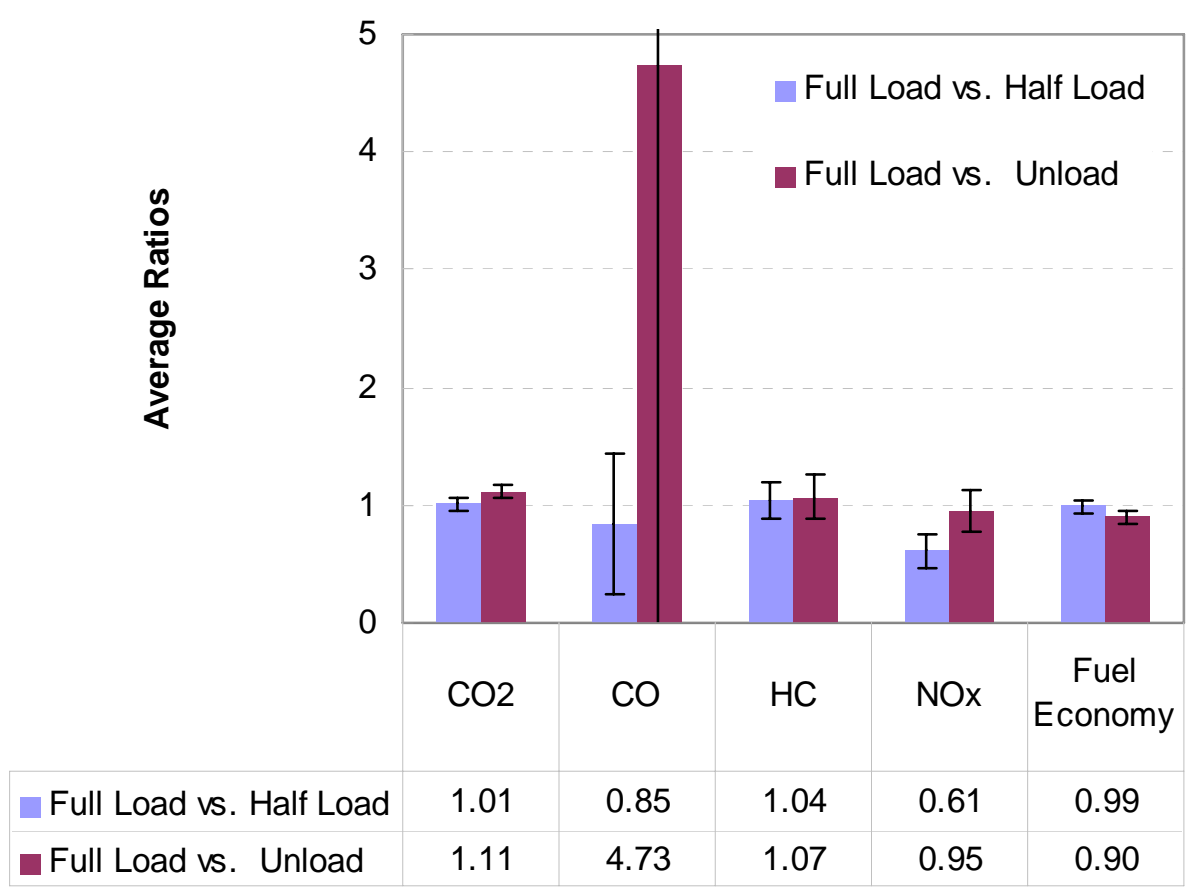

Figure 7.5 The Average Ratios of Emissions and Fuel Economy Based on Microtrip Data from a MY 2005 CNG Bus with a John Deere Engine Tested at Different Weights

\subsubsection{Road Load Equation}

To further investigate impact of vehicle weight on emissions and fuel economy, equation derivations based on the road load equation were conducted to determine the weight correction factors as a function of the average speed.

The road load equation is based on Newton's second law and it consists of three resistances: rolling resistance, aerodynamic drag, and grading resistance [100]. The power form of the road load equation could be expressed as: 


$$
\begin{aligned}
& P=\frac{V}{\eta_{t}}\left(F_{r}+F_{w}+F_{g}+M \delta \frac{d V}{d t}\right) \\
& F_{r}=f_{r} M g \cos \alpha \\
& F_{w}=\frac{1}{2} \rho A C_{d}\left(V+V_{w}\right)^{2} \\
& F_{g}=M g \sin \alpha
\end{aligned}
$$

Equation 7.2

Equation 7.3

Equation 7.4

Equation 7.5

where $P$ represented the power output of the engine, $\eta_{t}$ was the efficiency of drivetrain system, $F_{r}$ was the rolling resistance due to the contact between tires and ground, $F_{w}$ was the aerodynamic drag due to the wind, and $F_{g}$ was the grading resistance due to the slope. $M$ represented the vehicle weight, $\delta$ was the mass factor which represented equivalent mass increase due to the angular moments, $g$ was the gravity acceleration, $\alpha$ referred to the angle of the slope, $V$ was the vehicle speed, $t$ was the time, and $f_{r}$ was the rolling resistance coefficient. Equation 7.3 and Equation 7.5 show that a slope affects both rolling and grading resistance. In this study, it was assumed that grading resistance was neglected since the chassis dynamometer test did not account for the grade effect. Aerodynamic drag was affected by the frontal area of the vehicle as shown in Equation 7.4 where $\rho$ was the air density, $A$ was the frontal area of the vehicle, $C_{d}$ was the aerodynamic drag coefficient, and $V_{w}$ was the wind speed. Detailed introduction to the road load equation can be found in the reference by Ehsani et al. [100].

By combination from Equation 7.2 to Equation 7.5 and removal of grading resistance, the road load equation could be written as:

$$
\begin{array}{ll}
P=\frac{V}{\eta_{t}}\left(f_{r} M g+\frac{1}{2} C_{d} A\left(V+V_{w}\right)^{2}+M \delta \frac{d V}{d t}\right. & \text { Equation } 7.6
\end{array}
$$

To connect fuel consumption with engine power, fuel consumption rate was used and could be written as Equation 7.7.

$$
\begin{array}{ll}
F C R(t)=s f c \times P(t) & \text { Equation } 7.7
\end{array}
$$

where $s f c$ represented the specific fuel consumption per unit power output [99] and it varied depending on the operating condition of the engine. To be simplified, specific fuel consumption was assumed constant for the same vehicle at different weight. Thus, the integrated fuel consumption values of a duty cycle could be expressed as:

$$
F C=\int F C R(t) d t=s f c \int P(t) d t
$$

Equation 7.8 
Assuming that fuel consumption of a vehicle tested at a reference weight $M_{r e f}$ over a given duty cycle was $F C_{r e f}$, and then the difference in FC from the same vehicle but different weight $M$ could be written as:

$$
F C-F C_{r e f}=s f \mathcal{c} \int\left[P(t)-P_{r e f}(t)\right] d t
$$

\section{Equation 7.9}

It was assumed that

$$
\int V(t) d t=\bar{V} \Delta t \text { and } \sigma^{2}(V)=\overline{(V-\bar{V})^{2}}={\overline{V^{2}}}^{2} \bar{V}^{2}
$$

Equation 7.10

where $\bar{V}$ was the average speed and $\sigma(V)$ represented the standard deviation of the vehicle speed. In general, the drivetrain system transfers power at different efficiency rates depending on operating conditions of the vehicle. Higher efficiency values usually occur at higher load with higher gear ratios of the transmission. In this study, drivetrain efficiency was assumed to be constant for the same vehicle at different weight, as well as frontal area, aerodynamic drag coefficient, etc. In addition, the air density, wind speed and average speed were assumed constant for the vehicle. The rolling resistance coefficient could be calculated as a linear function of vehicle speed [99], then

$$
f_{r}=f_{r 1}+f_{r 2} \cdot V
$$

Equation 7.11

where $f_{r 1}$ was the intercept and $f_{r 2}$ was the slope. Both parameters were related to the tires pressure [99] and were assumed constant here. Equation 7.6, Equation 7.10 and Equation 7.11 were substituted into Equation 7.9, and then it could be simplified as:

$$
F C-F C_{r e f}=\frac{1}{\eta_{t}} s f c\left(M-M_{r e f}\right) g\left[f_{r 1} \bar{V}+f_{r 2}\left(\bar{V}^{2}+\sigma^{2}(V)\right)\right] \Delta t
$$

Equation 7.12

where $\Delta t$ represented the duration of the duty cycle, and $\sigma(V)$ represented the standard deviation of the vehicle speed. Equation 7.12 shows that the fuel consumption difference between different weight over a given cycle relates to average speed, standard deviation of vehicle speed as well as the duration of the cycle.

$$
F E=\frac{D}{F C}
$$

Equation 7.13

where $F E$ represented the fuel economy and $D$ represented the distance covered by the duty cycle. Equation 7.14 calculated the weight correction factor using the ratio of fuel economy of different weight to that of reference weight. After substituting Equation 7.12 into Equation 7.14, it could be written as Equation 7.15. 


$$
\begin{aligned}
& c_{w t . F E}(\bar{V})=\frac{F E}{F E_{r e f}}=\frac{F C_{r e f}}{F C}=\frac{\int F C R_{r e f}(t) d t}{\int F C R(t) d t}=\frac{\int P_{r e f}(t) d t}{\int P(t) d t} \\
& c_{w t . F E}(\bar{V})=\frac{1}{1+\frac{1}{F C_{r e f}} \cdot \frac{s f c}{\eta_{t}}\left(M-M_{r e f}\right) g\left[f_{r 1} \bar{V}+f_{r 2}\left(\bar{V}^{2}+\sigma^{2}(V)\right)\right] \Delta t}
\end{aligned}
$$

Equation 7.15

Equation 7.15 represented weight correction factor of fuel economy as a function of average speed. At a given duty cycle and vehicle, all parameters were available in the above equation. If assumed that

$$
Q(\bar{V})=\frac{1}{F C_{r e f}} \cdot \frac{s f c}{\eta_{t}} g\left[f_{r 1} \bar{V}+f_{r 2}\left(\bar{V}^{2}+\sigma^{2}(V)\right)\right] \Delta t
$$

Equation 7.16

then

$$
c_{w t . F E}(\bar{V})=\frac{1}{1+Q(\bar{V})\left(M-M_{r e f}\right)}
$$

It was found that $Q(\bar{V})$ could be approximately expressed as a linear function of average speed of different cycles. Thus it can be written as:

$$
Q(\bar{V})=k \bar{V}+b
$$

Equation 7.18

where $k$ was the slope and $b$ was the intercept. In this way, other parameters were removed from Equation 7.15 and only average speed was retained. The maximum and minimum average speeds from the database were used to calculate the values of $k$ and $b$. Due to the correction factors were always positive values when $M$ was smaller than $M_{\text {ref }}$ there was a limit value for vehicle weight $M$ to meet this condition.

To determine the weight correction factor of emissions, emissions rates were connected to power and calculated as:

$$
\operatorname{EmR}(t)=\operatorname{SEm} \times P(t)
$$

\section{Equation 7.19}

where SEm represented the specific emissions per unit power output [99] and was assumed constant for the vehicle at different weight. The correction factor of emissions could be expressed as: 


$$
c_{w t . E m}(\bar{V})=\frac{E m}{E m_{r e f}}=\frac{\int E m R(t) d t}{\int E m R_{r e f}(t) d t}=\frac{\int P(t) d t}{\int P_{r e f}(t) d t}
$$

Equation 7.20

Thus,

$$
c_{w t . E m}(\bar{V})=\frac{1}{c_{w t . F E}(\bar{V})}
$$

Equation 7.21 shows that weight correction factor of emissions is the reciprocal of that of fuel economy. Based on the impact analysis of vehicle weight from previous subsections, this relationship might be applied on $\mathrm{CO}_{2}$ and NOx emissions. For $\mathrm{HC}$, $\mathrm{CO}$ and PM emissions, the emission ratios between different weight did not illustrate clear trend with average speed. Thus, their correction factors might not be correlated with those of fuel economy.

To be simplified, multiple assumptions were made for the equation derivations based road load equation, such as constant values in drivetrain efficiency, specific fuel consumption and specific emissions for a vehicle with different weight. Combustions in an engine are a complex process of chemical reactions. The constant values for these parameters would be difficult to obtain in the real-world vehicle operations. As a result, Equation 7.17 and Equation 7.21 were used in this study to approximately estimate correction factors from different vehicle weight. Figure 7.6 and Figure 7.7 show example results of weight correction factors for fuel economy and emissions of the diesel backbone model in MY group 1998-2002. 


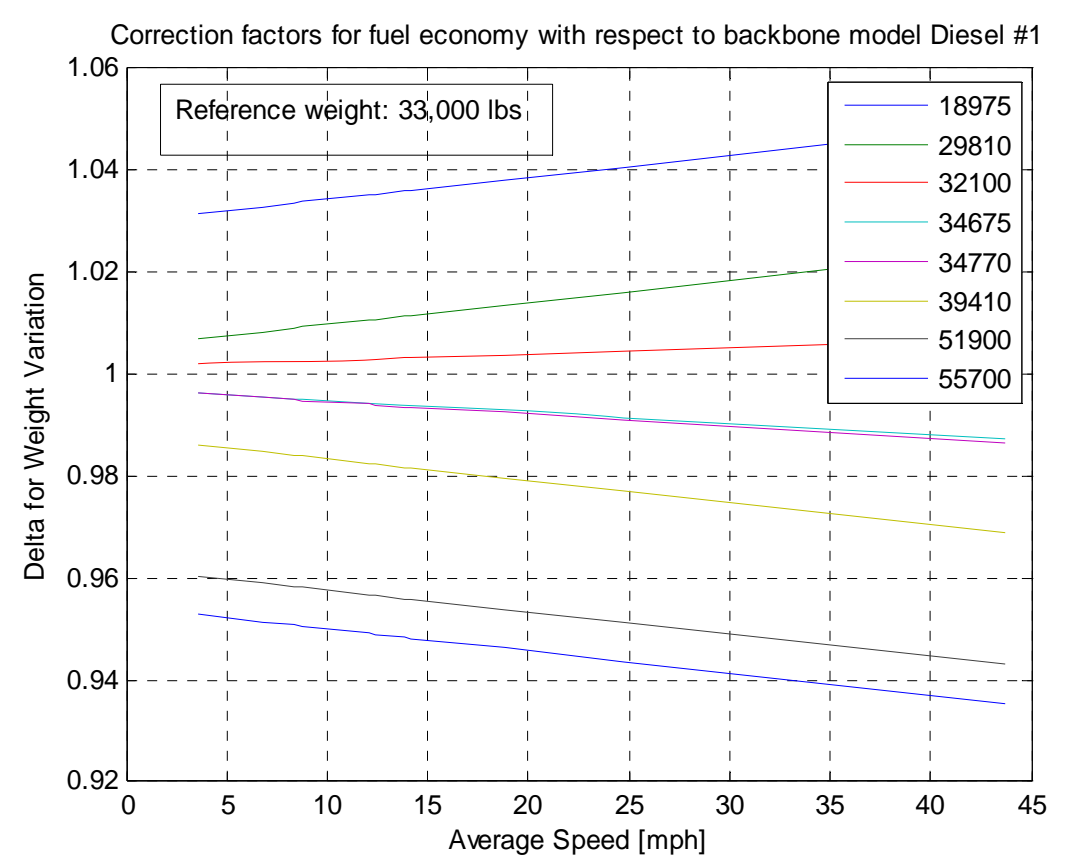

Figure 7.6 Weight Correction Factors for Fuel Economy as a Function of Average Speed for the Diesel Backbone Model of MY group 1998-2002

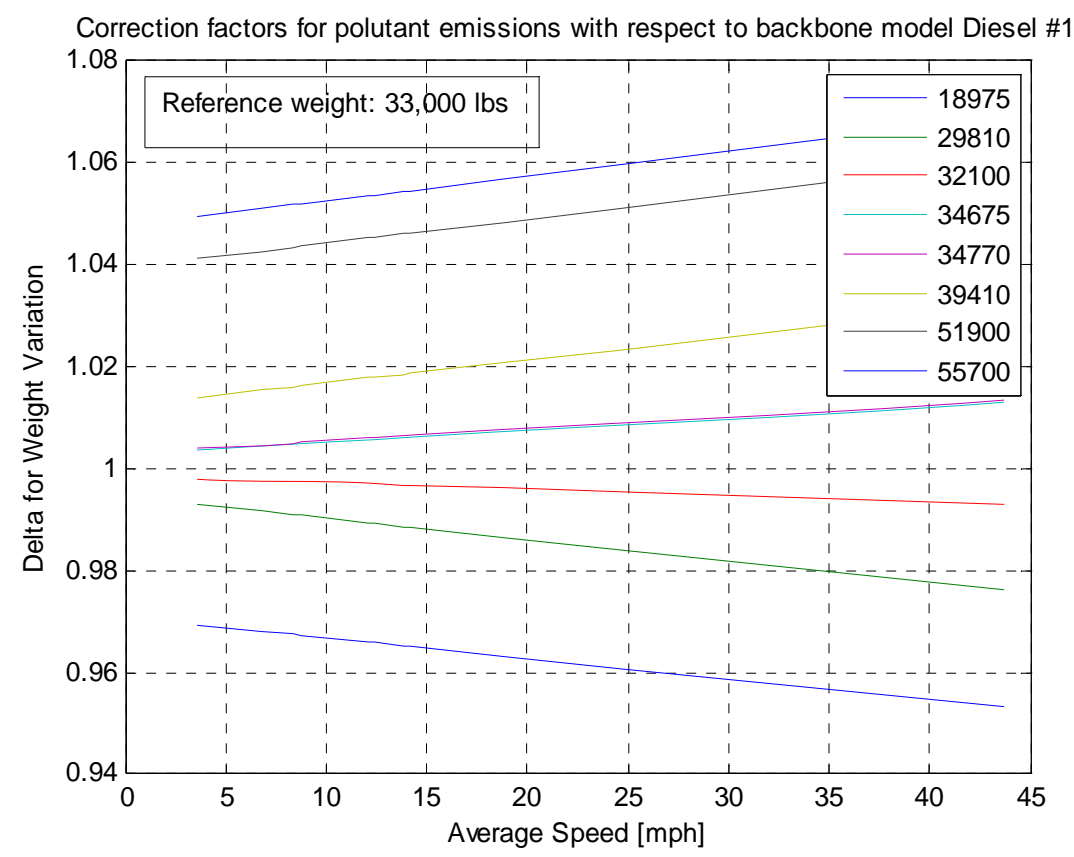

Figure 7.7 Weight Correction Factors for Emissions as a Function of Average Speed for the Diesel Backbone Model of MY group 1998-2002

\subsection{Model Year Group}

There is a large amount of emission data from the CBD cycle in the WVU database. In earlier MY groups, only the CBD cycle was performed for emissions tests. As a result, the CBD 
cycle was selected to determine ratios (correction factors) for emissions and fuel economy from older buses when they were compared to the newer backbone buses. In this section, the CBD ratios were determined for both diesel and CNG buses. In addition, ratios from a few other cycles and ratios as a function of average speed were investigated as well for comparison purposes.

\subsubsection{Diesel Bus}

Table 7.1 shows the number of diesel transit buses and test runs which were considered to determine CBD ratios for each MY group earlier than MY 1998. These buses were powered by D1, D2 or ULSD1 diesel fuel without the DPFs and they had test weights ranging from 30,050 to 35,925 lbs. To determine the CBD ratios, the test results on the CBD cycle from each MY group were averaged and then compared to those of the CBD cycle from the diesel backbone bus for MY Group IV (Table 6.1) which was tested on the same weight range as those older buses. The CBD ratios are shown in Figure 7.8 with error bars indicating their standard deviations at each MY group. It shows that on average the older buses had higher emissions and lower fuel economy. The CO, HC and PM emissions had large standard deviation values while the NOx and $\mathrm{CO}_{2}$ emissions as well as the fuel economy had relatively smaller standard deviation values. This is reasonable because CO, HC and PM emissions are very sensitive to the transient features of the operation conditions of the tested vehicles as well as the variations from the tested vehicles while the $\mathrm{NOx}, \mathrm{CO}_{2}$ and fuel economy are relatively steady and depend more on the steady characteristics of the test cycle such as average speed.

Table 7.1 Number of Diesel Buses Tested on CBD Cycle by Model Year Groups

\begin{tabular}{lccc}
\hline & $\begin{array}{c}\text { Number of Candidate } \\
\text { Buses }^{\mathrm{a}}\end{array}$ & Number of Different Test Runs $^{\mathrm{b}}$ \\
\hline MY Group & 39 & 50 \\
MY Group I & $1988-1991$ & 23 & 43 \\
MY Group II & $1992-1993$ & 21 & 21 \\
MY Group III & $1994-1997$ & Backbone IV & \\
MY Group IV & $1998-2002$ & Backbone V & \\
MY Group V & $2003-2006$ &
\end{tabular}




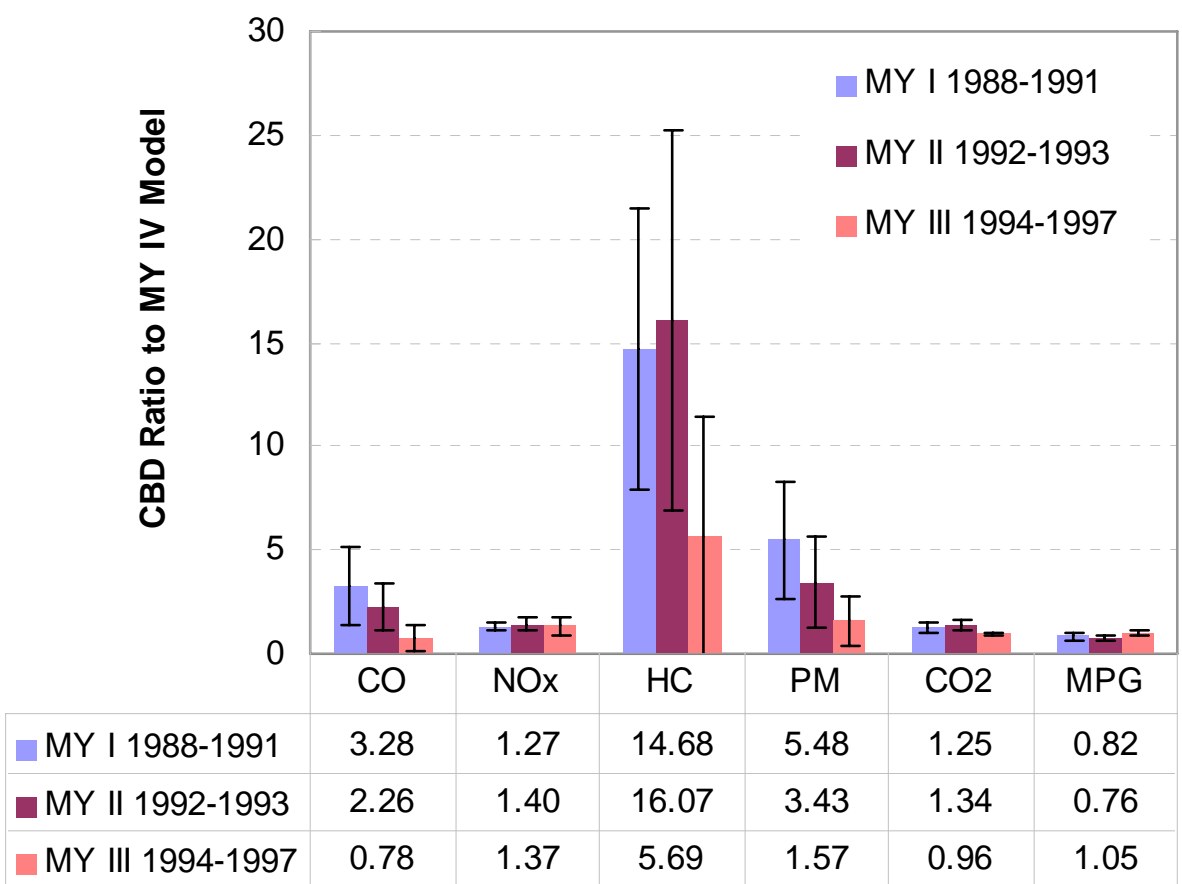

Figure 7.8 Average CBD Cycle Ratios from Other MY Groups Compared to MY 1998 - 2002 Diesel Backbone Model

To preliminarily validate the CBD ratio method, CBD ratios were applied to another MY group and projected results were compared to those of the emissions testing in that MY group. Figure 7.9 provides an example to show the results in MY group III by applying the CBD ratios to the values from the backbone MY IV diesel bus. The emissions testing data based on a limited number of cycles are shown in Figure 7.9 as well. It shows that the projected overall trends agreed with those of the test data points except for some test data points that lie off of the trends for $\mathrm{CO}$ and $\mathrm{HC}$ emissions, indicating that CBD ratio based MY group correction factors could be a good approach to approximately represent MY groups in which backbone models were not developed. 

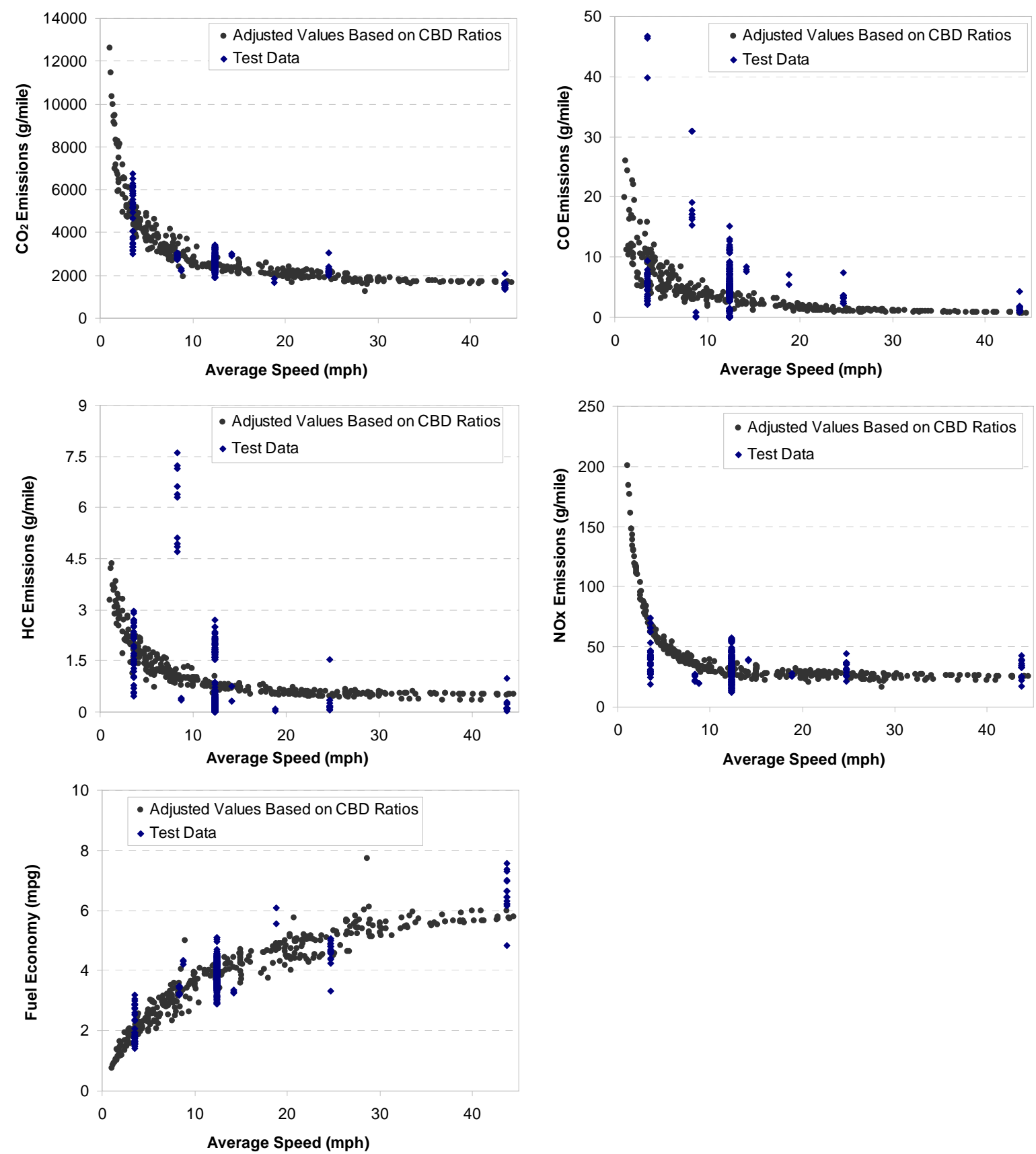

Figure 7.9 Emissions Testing Data of Diesel Buses from MY 1994 - 1997 and Adjusted Values Based on CBD Ratios from the Diesel Backbone Bus of MY 1998 - 2002

For comparison purposes, ratios from other cycles such as the NYBUS and WMATA cycles were studied as well. Figure 7.10 compares the NYBUS and WMATA ratios from transit buses in MY group III. These ratios were determined after the average values of test results were compared to the estimated values from the MY group IV diesel backbone model. Figure 7.10 shows that the ratios of $\mathrm{NOx}, \mathrm{CO}_{2}$, and fuel economy are comparable between the NYBUS and 
WMATA cycles. Especially, $\mathrm{CO}_{2}$ and fuel economy showed comparable results among the NYBUS, WMATA and CBD cycles, which might indicate that their ratios are independent from tested cycles.

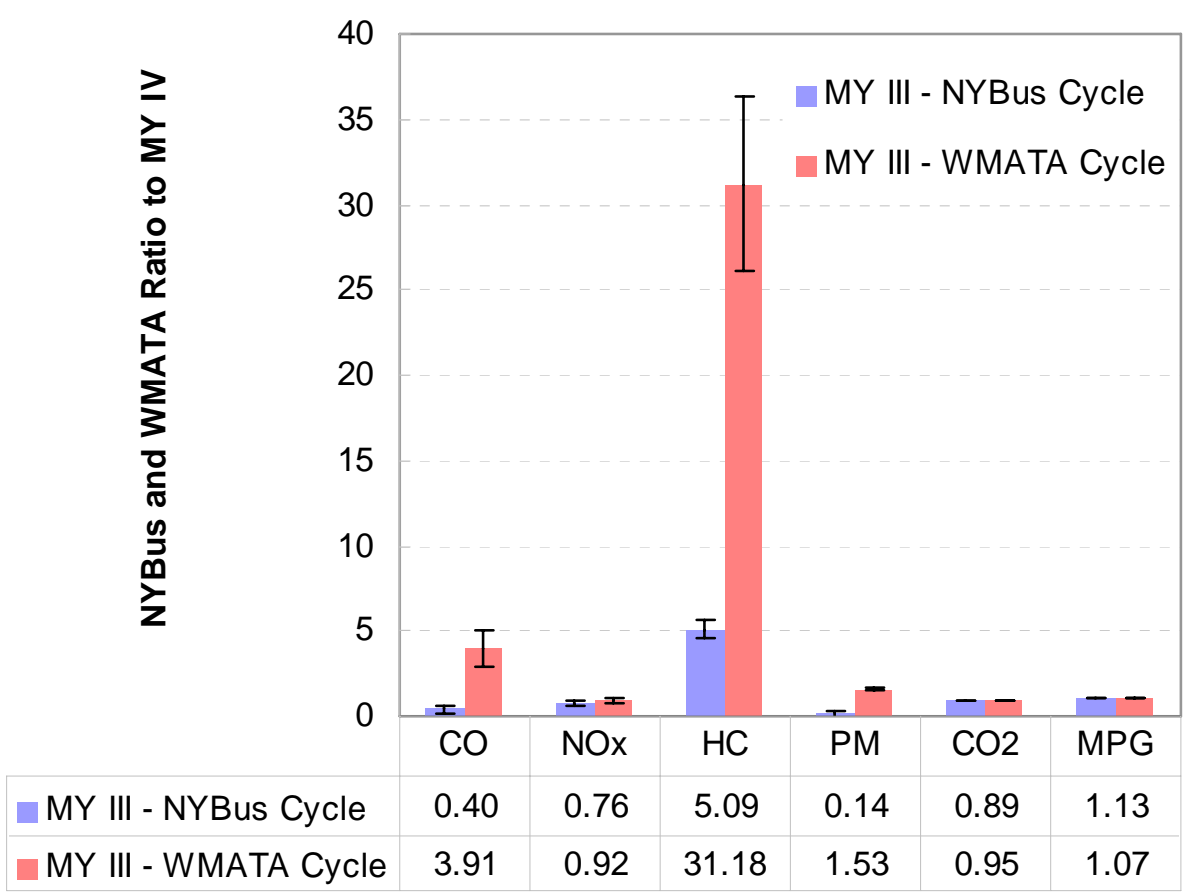

Figure 7.10 Average NYBus and WMATA Cycle Ratios from MY 1994 - 1997 Compared to MY 1998 - 2002 Diesel Backbone Model

To further investigate how different cycles or average speeds could affect ratios between MY groups, two backbone buses from different MY groups were selected and their emissions and fuel economy were compared. One diesel backbone bus was selected for each of MY group IV and $\mathrm{V}$ as shown in Table 6.1. The emissions and fuel economy data from these two buses were used to generate the same set of numerous virtual cycles for backbone modeling in each MY group. These two buses were comparable in terms of vehicle specifications except different model years. Due to the same cycles used for each bus to expand the database, it became possible to compare the emissions and fuel economy between the two MY groups as a function of average speed from standard cycles as well as virtual cycles.

Figure 7.11 illustrates the ratios as a function of average speed. Each data point refers to the ratio of the emissions or fuel economy value of MY group IV to that of MY group V. 

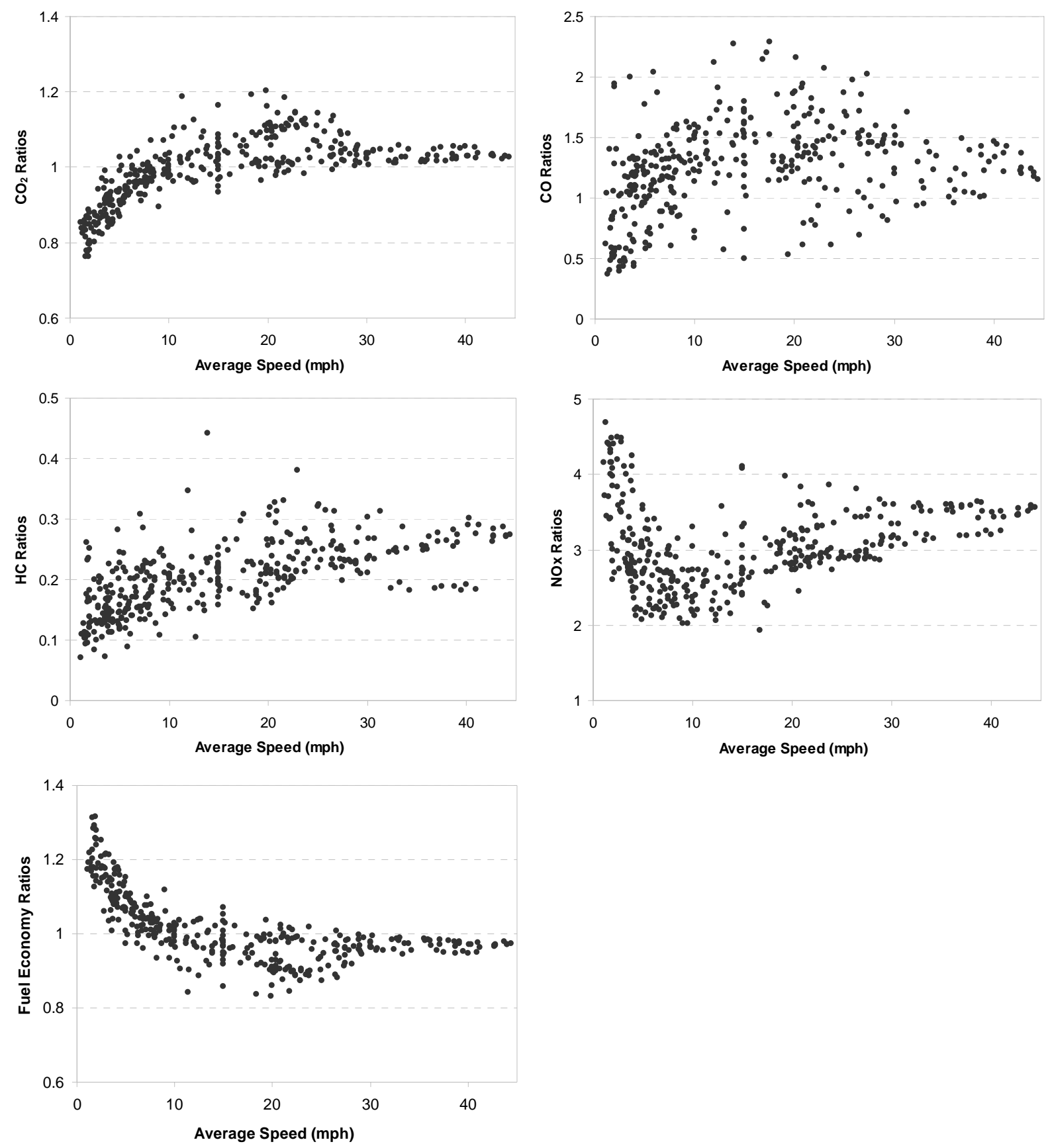

Figure 7.11 Emissions and Fuel Economy Ratios of MY 1998 - 2002 to MY 2003 - 2006 Diesel Backbone Bus as a Function of Average Speed

Figure 7.11 shows that $\mathrm{CO}_{2}$, NOx and fuel economy at lower average speed (about 10 mph and below) have different trends from higher average speed. The ratios at higher average speed are more independent from average speed and the trends are relatively flat as a function average speed, while at lower average speed the ratios change with average speed. This might be because at higher speed the cruise mode dominated and $\mathrm{CO}_{2}, \mathrm{NOx}$ and fuel economy became 
linearly correlated with the average speed while at lower average speed the transient mode had a major impact and the correlation with average speed was non-linear.

As a result, this study assumed constant ratios (CBD ratios) as correction factors and they were applied to $\mathrm{NOx}, \mathrm{CO}_{2}$ and fuel economy in each of older MY groups in which backbone models were not developed while the $\mathrm{HC}, \mathrm{CO}$ and PM emissions were temporarily considered as the same as those of the backbone model due to their significant variations.

\subsubsection{CNG Bus}

Table 7.2 shows the number of available candidate CNG buses tested on CBD cycles. These CNG buses had tested weight ranging from 31,950 to 39,940 lbs. Figure 7.12 shows average CBD ratios from MY I to MY IV based on the comparison with estimated values from MY V CNG backbone model. The approach to calculate CBD ratios was the same as diesel buses. The error bars indicate the standard deviations of the CBD ratios. Similarly, the CBD ratios of $\mathrm{NOx}, \mathrm{CO}_{2}$ and fuel economy had relatively smaller variance compared to other emissions. Compared to diesel buses, CNG buses had relatively smaller variation in HC emissions but substantially greater variation in $\mathrm{CO}$ emissions. The ratios and their standard deviations for CO emissions shown in Figure 7.12 have been divided by 10 from the original values. In some cases, different engine manufacturers or catalytic converter manufacturers had significantly different (10 folds) CO emissions when other conditions were similar.

Table 7.2 Number of CNG Buses Tested on CBD Cycle by Model Year Groups

\begin{tabular}{|c|c|c|c|}
\hline MY Groups & & Number of Candidate Buses $^{\mathrm{a}}$ & $\begin{array}{l}\text { Number of Different Test } \\
\text { Runs }^{\text {b }}\end{array}$ \\
\hline MY Group I & 1988-1991 & 11 & 16 \\
\hline MY Group II & 1992-1993 & 13 & 14 \\
\hline MY Group III & 1994-1997 & 24 & 31 \\
\hline MY Group IV & 1998-2002 & 14 & 14 \\
\hline MY Group V & 2003-2006 & Backbone V & - \\
\hline MY Group VI & 2007- & Backbone VI & - \\
\hline
\end{tabular}




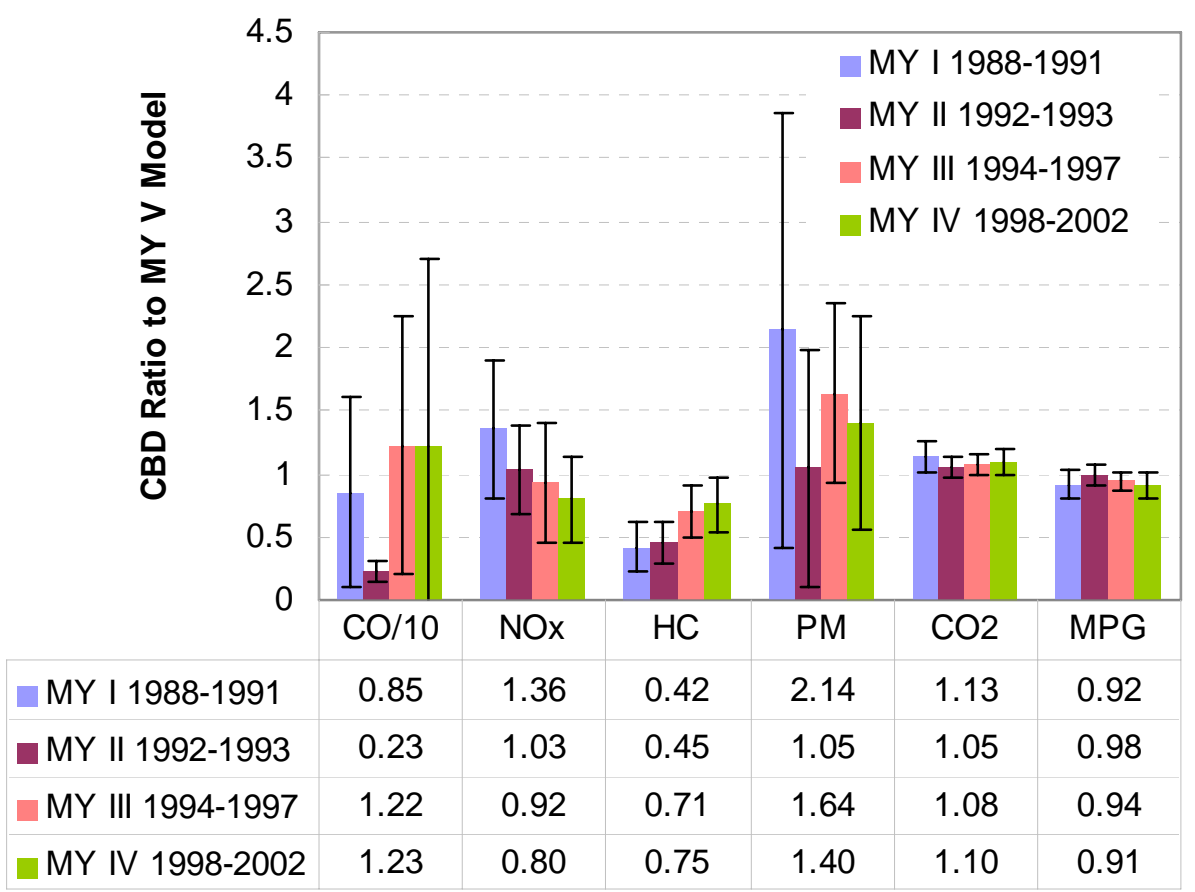

Figure 7.12 Average CBD Cycle Ratios from Other MY Groups Compared to MY 2003 - 2006 CNG Backbone Model

\subsection{After-Treatment Technologies}

Data analysis was performed for diesel transit buses with and without after-treatment technologies and the correction factors were determined. Many after-treatment technologies could be used to effectively reduce the emissions. The diesel oxidation catalyst (DOC) and diesel particulate filters (DPF) have been used to reduce HC, CO and PM emissions from diesel vehicles while the exhaust gas recirculation (EGR) and selective catalytic reduction (SCR) could be used to reduce NOx emissions. These technologies can be combined to control the emissions.

Due to more stringent emissions standard required by the EPA for highway engines and vehicles in model year 2007 and beyond [101], after-treatment technologies must be employed to reduce emissions. The high sulfur content in the diesel fuel might damage the emissions reduction equipment and adversely affect their effectiveness. The EPA required the sulfur content of diesel fuel to be reduced to 15 parts per million (ppm) for on-road vehicles and 15 ppm ultra-low sulfur diesel (ULSD) was required to be produced starting in mid-2006 [101].

The DPF is one of most effective emissions reduction technologies and it has been used as a retrofit technology on the older vehicles and helps them become cleaner transportation. 
In this study, the DPF was selected to determine the correct factor between transit buses with and without a DPF. In total, 11 transit buses were selected and each of these buses was tested on the same cycles with and without the DPF. All of these buses had model year no later than 2002 and they were tested after 2002 with test weight ranging from 18,975 to 51,900 lbs. Engine manufacturers of these vehicles included Cummins and Detroit Diesel, and DPF manufacturers included Engelhard, Johnson Matthey, and Nelson. The tested chassis dynamometer cycles included BEELINE and WMATA cycles and ultra-low sulfur diesel type 1 (ULSD1) fuel was used on those bused for the emissions testing.

Figure 7.13 shows the average percent reduction in terms of emissions and fuel economy from these buses with the error bars indicating the minimum and maximum values. Reduction percentage was determined based on the comparison between the test results with and without DPF. Except the emissions control device, the other test conditions were intended to be same for each bus (i.e. test cycles, fuel type, testing weight, test period, etc). As a result, the difference between test results might be primarily attributed to the DPF device used on the bus.

Figure 7.13 shows that the DPF has substantial effect on CO, HC and PM emissions with on average reduction of $86.4 \%, 95.8 \%$ and $92.2 \%$, respectively. The DPFs did not significantly affect the NOx emissions while on average they slightly affected fuel economy (2.6\% reduction) as a penalty. These results fall between or even are a little better than the ranges indicated by the EPA for the verified DPFs which were estimated at 85 to $90 \%$ or beyond for PM reduction and 70 to $90 \%$ for CO and HC reduction while usually none for NOx reduction [102].

In addition, Figure 7.13 shows that due to different buses as well as different DPF manufacturers there was some variation (maximum difference) with respect to the effect of the DPFs such as $35 \%, 18 \%, 33 \%, 24 \%, 30 \%$ and $26 \%$ for $\mathrm{CO}, \mathrm{HC}, \mathrm{NOx}, \mathrm{PM}, \mathrm{CO}_{2}$ and fuel economy, respectively.

The diesel backbone IV bus (MY 2000) and diesel backbone V (MY 2006) were not equipped with a DPF. As a result, based on the diesel bus backbone models, these correction factors could be used to estimate emissions from the same MY or older diesel buses retrofitted with a DPF. 


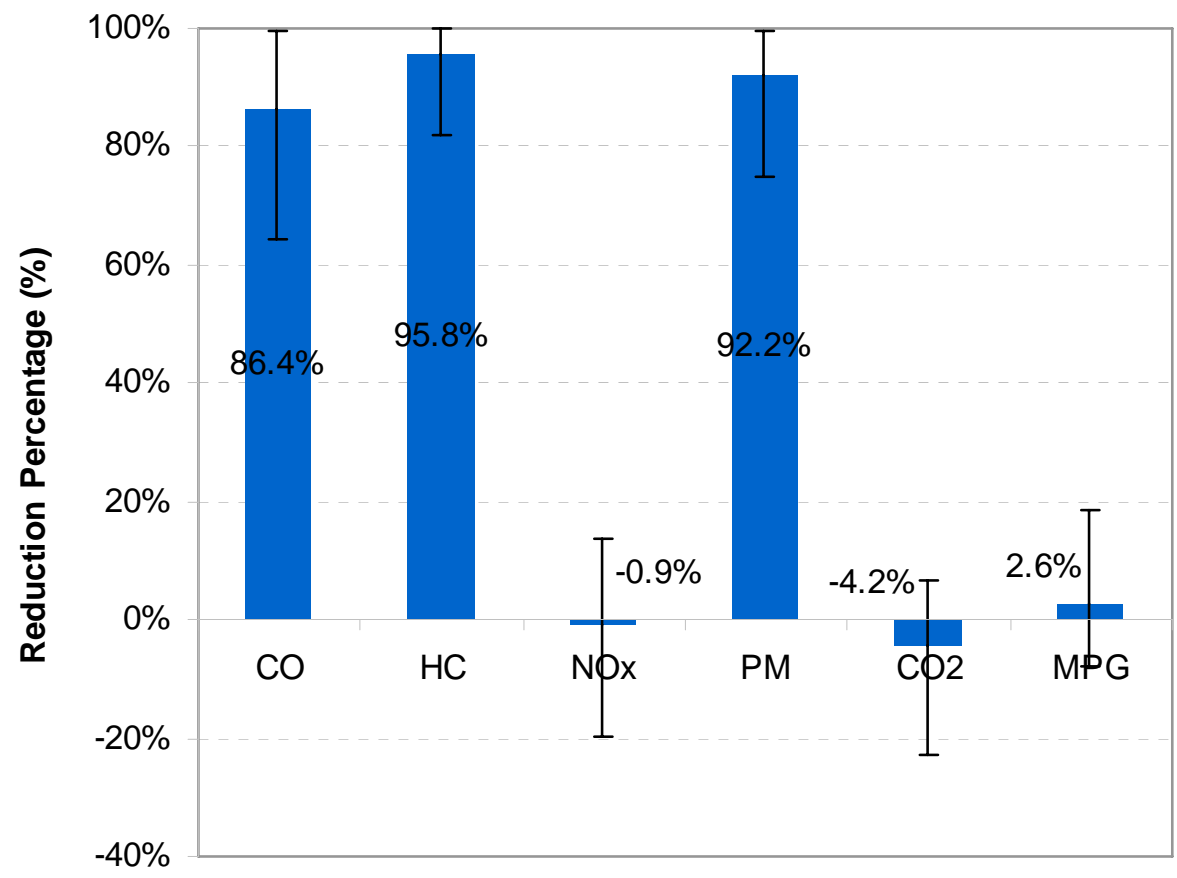

Figure 7.13 Average Emissions and Fuel Economy Reduction Percentage of Diesel Buses with After-treatment Technologies 


\section{CASE STUDY}

A previous comparison study of the IBIS emissions model with EPA's MOBILE6 and MOVES models showed that HC, CO, and NOx emissions presented good agreement while PM emissions had acceptable agreement [103]. Regarding $\mathrm{CO}_{2}$ emissions and fuel economy, MOBILE6 output them as constant values while MOVES presented similar trends to the IBIS model but with significantly lower values for $\mathrm{CO}_{2}$ and higher values for fuel economy [103].

As a case study, this chapter compares the IBIS emissions model with CARB's EMFAC model. The latest version EMFAC2011 was used and model outputs were provided by the webbased data access tool $[9,10]$. For the EMFAC model, California statewide and Orange County (OC) as well as diesel powered urban buses were selected for the following comparisons.

The EMFAC model employed 14 speed bins ranging from 5 to $70 \mathrm{mph}$ with an increment of $5 \mathrm{mph}$ and required users to select end values of these speed bins (e.g. $5 \mathrm{mph}, 10 \mathrm{mph}, 20 \mathrm{mph}$, etc) as speed inputs for the model. For emissions outputs, running emission rates were considered in this study. Fuel economy was calculated based on carbon balance from the exhaust. For PM emissions, PM2.5 was selected to compare with IBIS outputs. EMFAC output hydrocarbons with different forms such as total organic gases (TOG) and reactive organic gases (ROG). A ratio of TOG to THC was taken from the EPA document regarding HC conversion factors [104] to obtain the HC values from EMFAC.

For comparison purposes, the IBIS model was run with the same sets of speed bin end values as the single average speed input. In addition, five cycle metrics were input into the IBIS model for 12 standard reference cycles. Figure 8.1 compares the model outputs for MY 2000 diesel buses in calendar year 2005 which represent diesel buses in MY Group IV (1998 - 2002) for the IBIS model. It shows that statewide and Orange County outputs from the EMFAC model nearly overlap. The IBIS model agreed fairly well for CO emissions with the EMFAC model. NOx and PM emissions presented similar trends between both models with increasing speed. At speed of $15 \mathrm{mph}$ and above NOx emissions from IBIS were higher than EMFAC's values while at speed of $15 \mathrm{mph}$ and below PM emissions from IBIS were lower than EMFAC's values. HC emissions from EMFAC were significantly higher than IBIS and differences became larger with decreased average speed. Regarding $\mathrm{CO}_{2}$ emissions, EMFAC output a constant value which was 
comparable to the average of IBIS's outputs at the same set of speeds. At an average speed of 15 mph, the two models shared about the same $\mathrm{CO}_{2}$ value while below $15 \mathrm{mph}$ IBIS was higher and above 15 mph EMFAC was higher. Fuel economy was nearly a constant value for EMFAC since its value was dominated by constant $\mathrm{CO}_{2}$ emissions when calculated by carbon balance.
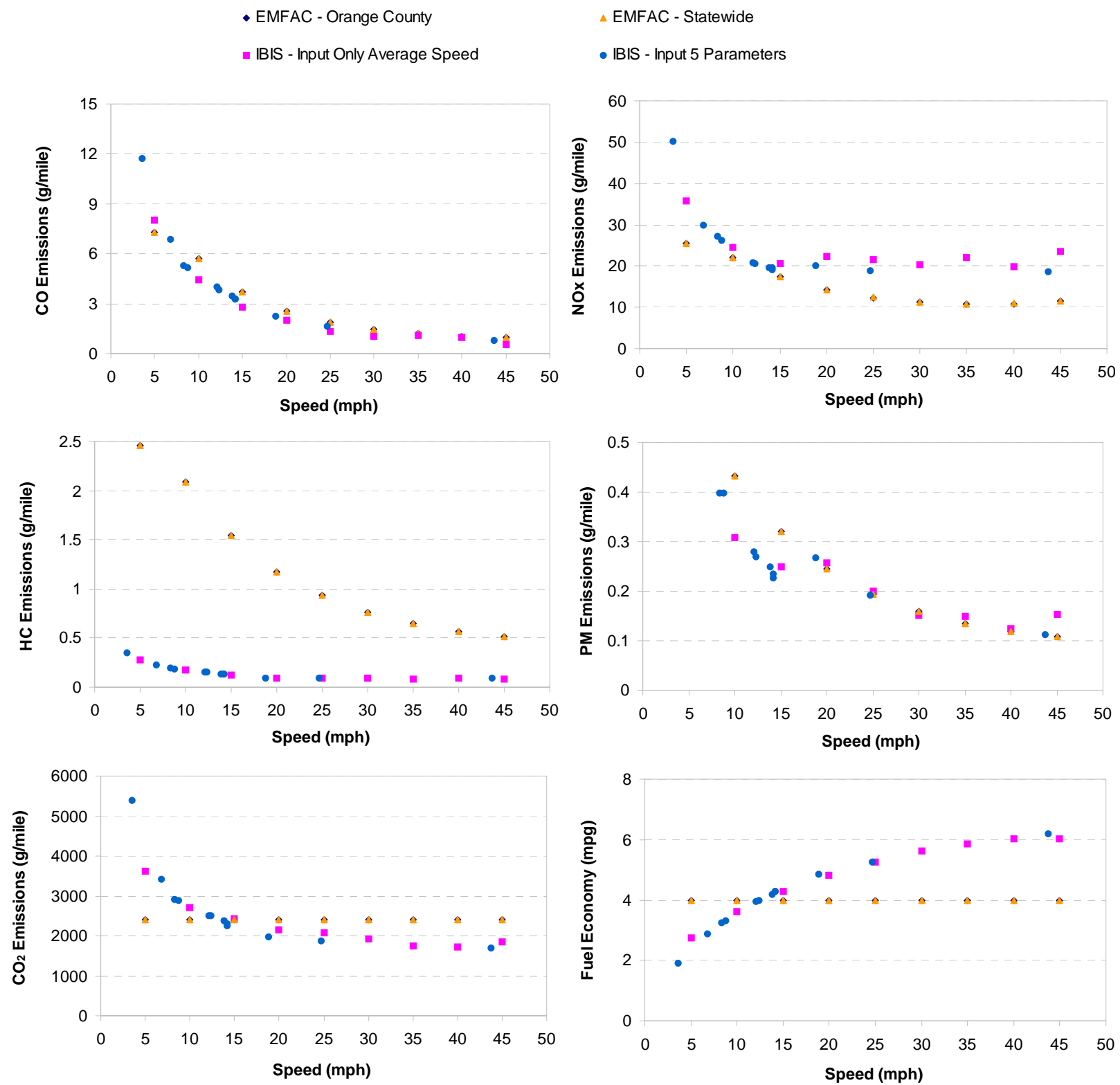

Figure 8.1 Comparisons of the EMFAC and IBIS Models for MY 2000 Diesel Buses in Calendar Year 2005

Figure 8.2 compares outputs of the EMFAC and IBIS models for MY 2006 diesel buses in calendar year 2006 which represent diesel buses in MY Group V (2003 - 2006) for the IBIS model. Similarly, the EMFAC shows the same results between California statewide and OC 
region for emissions and fuel economy. IBIS agreed well with EMFAC for CO emissions while IBIS showed higher NOx emissions as a function of average speed. At speeds of $25 \mathrm{mph}$ and above, IBIS had a good agreement with EMFAC for HC emissions while at speed below 25 mph IBIS presented significantly higher HC emissions and differences between the two models became larger with decreased speed. Regarding PM emission, IBIS presented higher values at most speeds. Similarly, $\mathrm{CO}_{2}$ emissions from EMFAC showed a constant value which shared the same value as those of MY 2000 diesel buses as discussed above and this value could compare with the average of IBIS outputs across the same set of speeds. IBIS showed increased fuel economy with increased speed while EMFAC showed constant fuel economy as a function of speed. The two models shared about the same fuel economy value at the speed of $10 \mathrm{mph}$. 

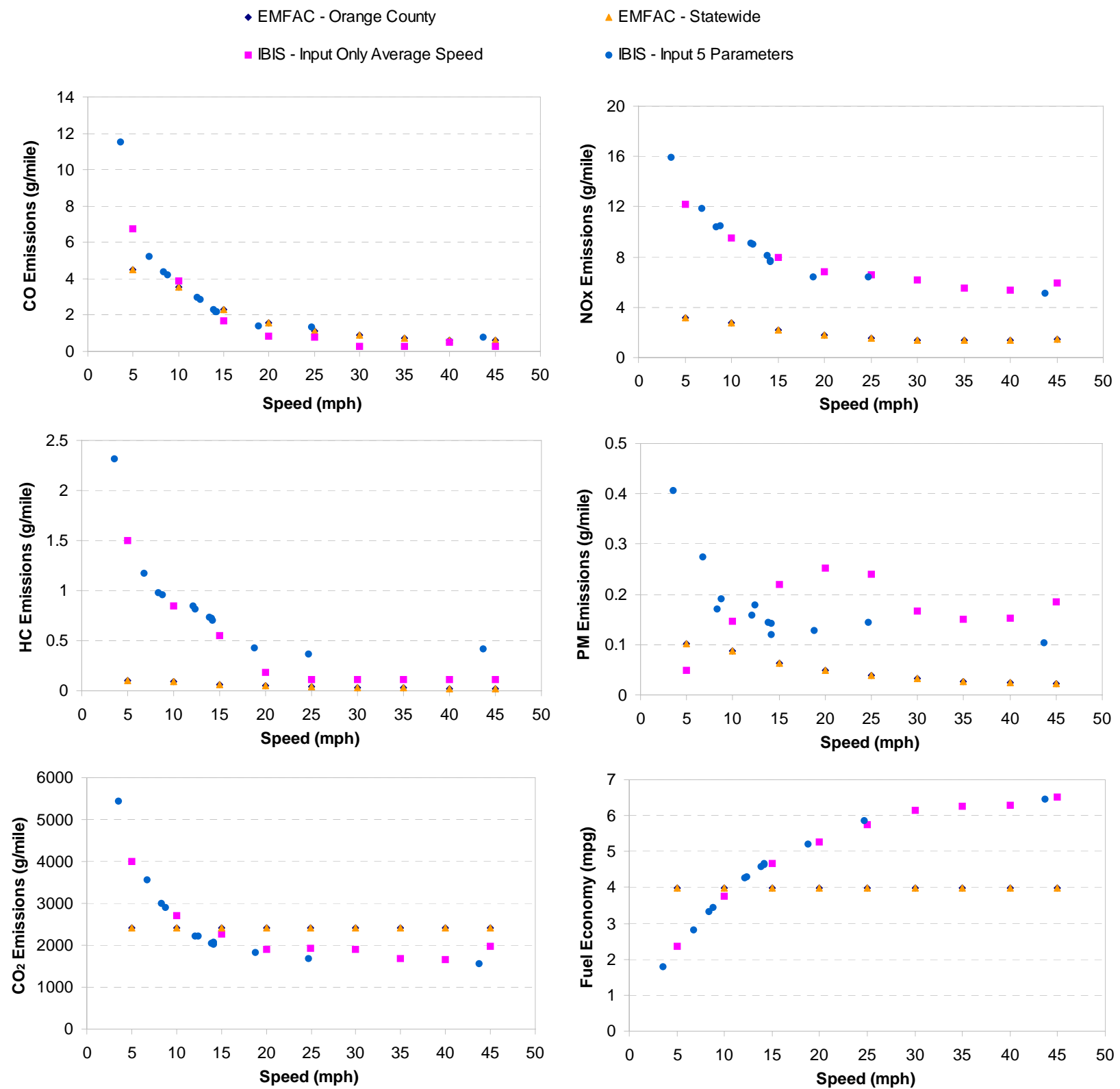

Figure 8.2 Comparisons of the EMFAC and IBIS Models for MY 2006 Diesel Buses in Calendar Year 2006

Figure 8.3 shows the results from two models for MY 2000 diesel buses as a function of calendar years. Results at two speeds (10 and $30 \mathrm{mph})$ were provided. It was intended to demonstrate how deterioration affected emissions and fuel economy from the EMFAC model when vehicles were getting older. It shows that emissions and fuel economy do not change with increased years.

However, the EMFAC model did account for the deterioration impact. Generally, when a vehicle ages it produces more emissions and consumes more fuel. The EMFAC2007 model 
employed vehicle cumulative mileage to reflect deterioration and assumed cumulative mileage as a function of mileage accrual over time [10]. However, older and higher mileage vehicles might have higher scrappage rates than lower mileage vehicles and thus travel fewer miles than those when they were newer [10]. As a result, EMFAC2011 measured vehicle cumulative mileage as a function of the product of mileage accrual rates and vehicle survival rates. After considering both rates, the deterioration overall did not significantly affect emissions and fuel economy with increased vehicle ages.

The IBIS emissions model did not consider the deterioration impact as vehicles get older. Based on EMFAC's investigation in terms of the deterioration impact as described above, IBIS's assumption was reasonable. 

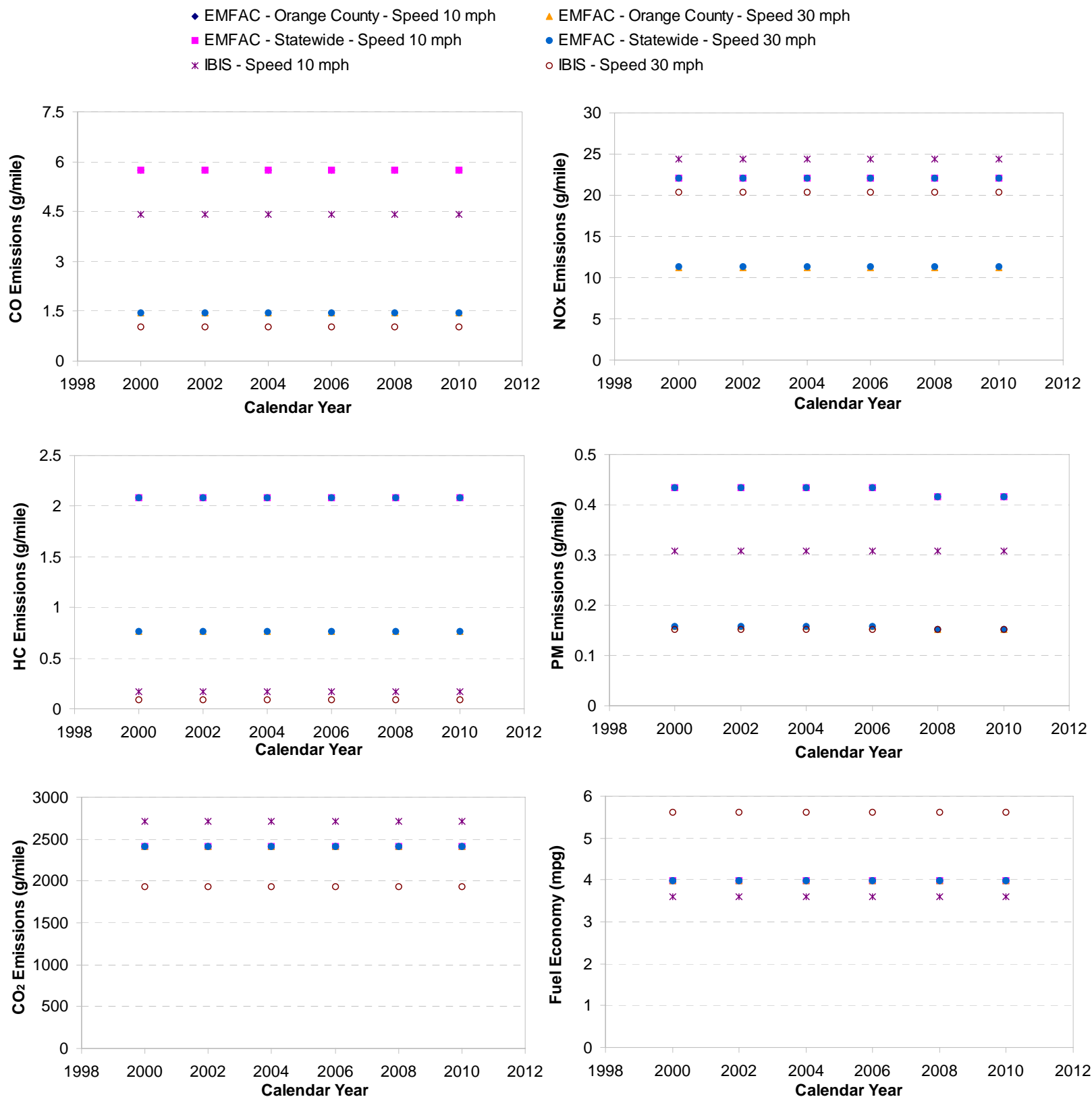

Figure 8.3 Comparisons of the EMFAC and IBIS Models for MY 2000 Diesel Buses from 2000 to 2010

Figure 8.4 illustrates results for diesel buses from the EMFAC model as a function of model years. It was intended to demonstrate how the EMFAC model was affected by model years. Model years in the EMFAC model reflected technology groups as well as emissions standards in California [8, 10]. For example, NOx and PM emission trends in Figure 8.4 show reduced values after MY 2002, which might reflect the regulation adopted by CARB and applied to transit buses in 2002. The regulation called for use of low sulfur diesel fuel, retrofit of older 
diesel buses with DPFs, as well as adoption of zero emission buses [105]. Figure 8.4 demonstrates overall reduced emissions and improved fuel economy with newer vehicles while different patterns were observed for different outputs. IBIS applied the same MY groups to all outputs and MY groups from the IBIS model reflected emission standards regulated by the EPA. In addition, it shows at two different speeds EMFAC has the same $\mathrm{CO}_{2}$ and fuel economy values and the values slightly change with increased model years.
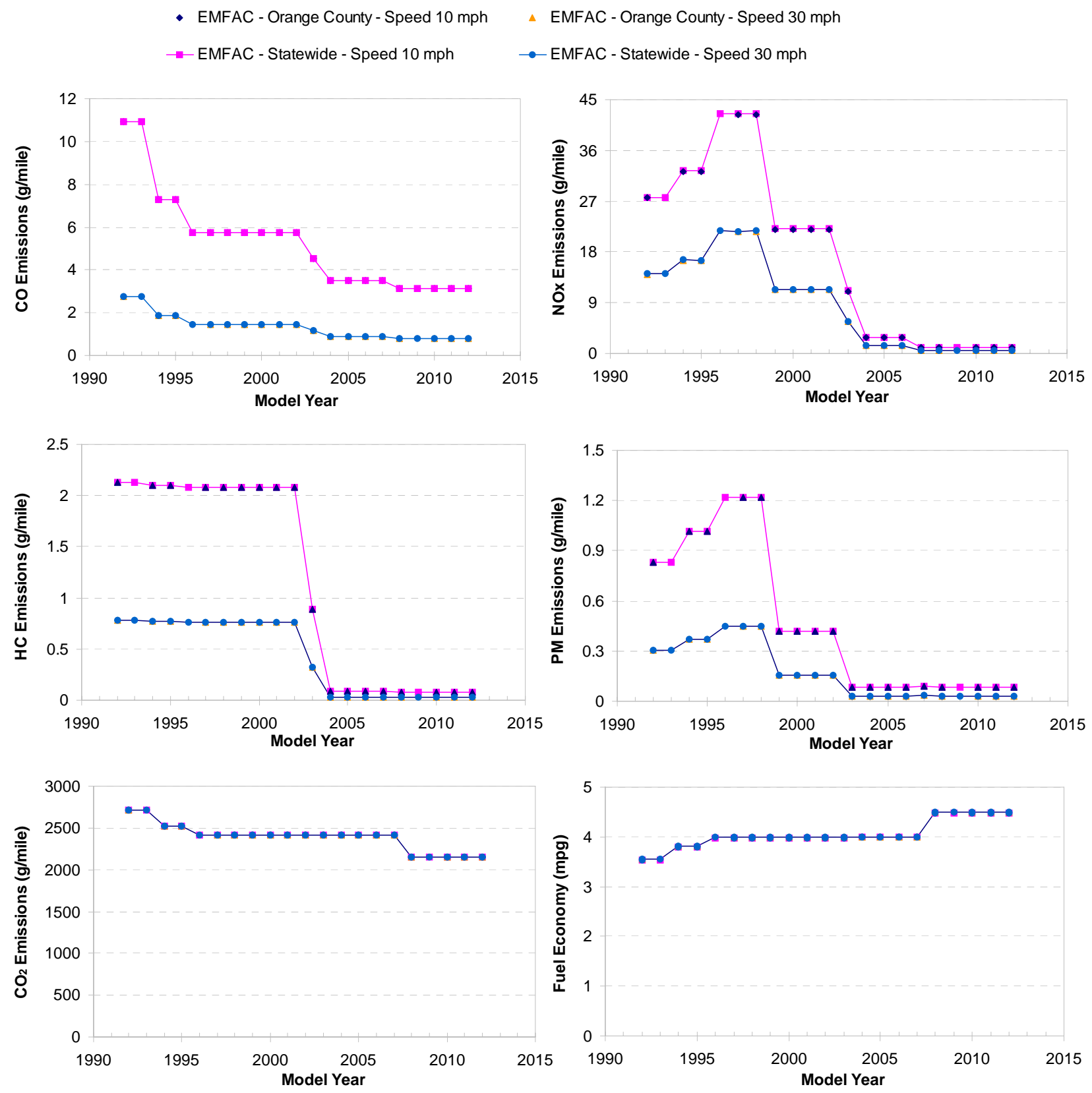

Figure 8.4 The EMFAC Model for Diesel Buses from MY 1992 to 2012 


\section{CONCLUSIONS AND RECOMMENDATIONS}

Through the investigation of emissions and fuel economy for the IBIS project, five major studies were conducted, including a correlation and regression study of cycle characteristics, virtual cycle and emissions generation and preliminary validation, development and validation of backbone models, development of correction factors, and a case study to compare the IBIS emissions model to CARB's EMFAC model. This chapter summarizes those major findings based on results and discussions from the tasks listed above. Finally, recommendations for the future research are provided.

\subsection{Conclusions}

\subsubsection{Correlation and Regression Study of Cycle Characteristics}

It has been well known that cycle characteristics affect emissions and fuel economy of the vehicle. A correlation and regression study was conducted to assess effects of cycle metrics (parameters) on emissions and fuel economy. Thirteen cycle metrics were calculated and their correlations between each other as well as correlations of some metrics with emissions and fuel economy were evaluated and significance levels were provided. In addition to regular correlation analysis, due to nonlinear relationships between some metrics and emissions, non-parametric correlation analysis was conducted. During the correlation analysis, multiple factors were considered to determine if certain cycle metrics should be retained or removed for emissions modeling. These factors included collinearity among cycle metrics, strength and significance of correlations with emissions and fuel economy and their ease of acquisition by transit agencies. Then, the five most influential cycle metrics for modeling purposes were determined to be average speed with idle, percentage idle, stops per mile, standard deviation of vehicle speed and kinetic intensity.

Preliminary regression analysis compared average speed based single parameter models with multiple parameter models based on selected metrics. It was found that multiple parameter models showed significant improvement in terms of higher $R$ squared values and lower root mean square errors. The multiple parameter models were calibrated against experimental results from the NYBus cycle, showing over 75\% reduced percentage errors for all responses when 
compared to average speed based models. In addition, multiple parameter models reduced mean percentage errors (MPE) after considering all cycle points in the regression study. The MPE reduction values were $68.6 \%$ for $\mathrm{CO}_{2}, 1.5 \%$ for $\mathrm{CO}, 50 \%$ for $\mathrm{HC}, 62.7 \%$ for $\mathrm{NOx}, 44.5 \%$ for fuel economy, and 13\% for PM. These further demonstrated how adding selected metrics to average speed based models improved the regressions models.

The results of this study assisted in determining appropriate strategies for later development and implementation of the IBIS emissions model. It provided a useful framework for the selection of the most influential cycle metrics for modeling.

\subsubsection{Virtual Cycles, Emissions Generation and Preliminary Validation}

Over 350 virtual cycles and associated emissions and fuel economy data were generated to fill sparse areas in the cycle parameter planes. It demonstrated that the cycle generation method could be employed to transit buses tested over the limited number of cycles and substantially expand the database for emissions and fuel economy modeling.

Curve fitting based on the expanded data points demonstrated that average speed played a major role to estimate response variables with a coefficient of determination above 0.91 for all except NOx emissions (0.88). This provided general strategies to select influential polynomial terms during the development of the IBIS backbone models. However, wider variation in certain ranges of average speed on the cycle parameter planes reinforced that average speed based single parameter models might not be able to fully estimate the response variables.

Emissions testing was conducted on selected virtual cycles for a MY 2008 CNG bus with a TWC and cooled EGR and a MY 2008 hybrid bus with a DOC and DPF. The selected virtual cycles were realistic since the driver could closely follow the target speed-time traces and the parity plots for actual speeds versus target speeds showed $R$ squared values of 0.99 or above and slope values of 0.96 or above.

Integrated values of fuel economy and $\mathrm{CO}_{2}$ emissions could be accurately represented using cycle and emission generation results within $1 \%$ and $2 \%$ respectively for the CNG bus and within 5\% for the hybrid bus. For both CNG and hybrid buses, second-by-second fuel consumption and $\mathrm{CO}_{2}$ rates could be relatively accurately (slope values of 0.89 or above) and precisely ( $R$ squared values of 0.78 or above) represented by the generation method. 
The preliminary results did not show that $\mathrm{HC}$ and $\mathrm{CO}$ emissions rates and integrated values could be accurately represented by the cycle and emissions generation method for both CNG and hybrid buses.

The stoichiometric CNG bus with a TWC and cooled EGR presented low levels of NOx emissions. Compared to the CNG bus, the hybrid bus presented significantly higher NOx emissions and NOx emissions were more accurately and precisely represented by the cycle generation results.

Regarding the impact of microtrip history, preliminary results showed that fuel economy, $\mathrm{CO}_{2}$ and NOx emissions were not sensitive to microtrip history. Microtrip history could affect the performance of aftertreatement devices. Thus, it could significantly affect emissions such as $\mathrm{CO}$ and $\mathrm{HC}$. Limted repeat tests further demonstrated that the $\mathrm{HC}$ and $\mathrm{CO}$ were highly sensitive to the transient features of vehicle operations and the small variability from test runs could lead to substantially different $\mathrm{HC}$ and $\mathrm{CO}$ emission levels.

\subsubsection{Backbone Model Development and Validation}

Based on expanded emissions data, backbone models were developed for buses of different types of fuel or propulsion systems in specific MY groups. For each response variable, four sub-models were developed and each sub-model was a polynomial regression model based on two cycle parameters from the five selected cycle parameters. Average speed was required as a mandatory input due to its dominant role in modeling.

Based on the characteristics of the available emissions testing data, as an example, two types of sub-models were developed for a MY 2008 CNG bus and a MY 2008 hybrid bus, including models based on both tested standard cycles and generated virtual cycles, and models based on only tested standard cycles with repair algorithms. Cycle generation was demonstrated as an effective method to expand the emissions database, but it was not always useful for modeling purposes, especially when the original experimental data did not show a trend or pattern. The cycle generation method could be employed to expand the size of the dataset but it was not supposed to change and improve the characteristics of the original data.

With the additional dataset, two-parameter and three-parameter polynomial models were compared and validated. For the CNG bus, validation errors of two cycle parameter models showed 6-7\% for fuel economy, 8-9\% for $\mathrm{CO}_{2}, 16-19 \%$ for $\mathrm{CO}$, and $23-34 \%$ for $\mathrm{HC}$ while those 
with three cycle parameters showed 6\% for fuel economy, 6-8\% for $\mathrm{CO}_{2}, 16-18 \%$ for $\mathrm{CO}$, and 22-29\% for HC. For the hybrid bus, validation errors of two cycle parameter models showed 7$8 \%$ for fuel economy, $7-10 \%$ for $\mathrm{CO}_{2}$, and $7-19 \%$ for $\mathrm{NOx}$ while those with three cycle parameters model showed 7\% for fuel economy, 8-10\% for $\mathrm{CO}_{2}$, and 7-17\% for NOx. As a result, it indicated that adding other parameters might not significantly improve the backbone model and average speed based two parameter backbone models could estimate and predict the response variables with acceptable accuracy.

\subsubsection{Development of Correction Factors}

In addition to driving characteristics (cycle metrics), other non-cycle parameters were investigated and related correction factors were developed and applied to the backbone models to improve the IBIS emissions model.

To investigate weight impact, microtrip data were extracted from a few standard cycles tested on the same vehicles with different weights so the effect of weight could be evaluated as a function of average speed. For the diesel bus tested on three weights, fuel economy, $\mathrm{CO}_{2}$ and NOx illustrated clear trends. On average, a $14 \%$ increase in weight could increase $\mathrm{CO}_{2}$ emissions by $6 \%$ and reduce fuel economy by $6 \%$. A $32 \%$ increase in weight could increase $\mathrm{CO}_{2}$ emissions by $17 \%$ and reduce fuel economy by $14 \%$. As a result, a W\% increase in test weight could roughly increase $\mathrm{CO}_{2}$ emissions and reduce fuel economy by $0.5 \mathrm{~W} \%$. For NOx emissions, a $14 \%$ increase in weight on average did not significantly affect the emissions while a $32 \%$ increase in weight resulted in a $12 \%$ increase in NOx emissions. The small standard deviation of weight ratios across all microtrips and standard test cycles for $\mathrm{CO}_{2}$, $\mathrm{NOx}$ and fuel economy indicated that the impact of weight on them was not sensitive to average speed of the vehicle. For CO, HC and PM, the points of weight ratio scattered and did not show clear trends, thus it was not possible to draw a conclusion in terms of the effects of weight. For the CNG bus, on average, a $12 \%$ weight increase did not significantly increase $\mathrm{CO}_{2}$ and fuel consumption while a 28\% weight increase increased $\mathrm{CO}_{2}$ by $11 \%$ and reduced fuel economy by $10 \%$. The CNG bus showed trends of minor weight impact for HC. A 12\% increase in weight on average increased HC by $4 \%$ while a $28 \%$ increase in weight on average increased HC by $7 \%$. Similar to the diesel bus, CO and PM for the CNG bus did not show clear trends. 
To further investigate the impact of vehicle weight on emissions and fuel economy, equation derivations based on the road load equation were conducted. The road load equation based weight correction factors were developed as a function of average speed and implemented into the IBIS emissions model for $\mathrm{CO}_{2}$, $\mathrm{NOx}$ and fuel economy.

Regarding correction factors with respect to MY groups, CBD ratios were determined for MY groups in which backbone models were not developed. Preliminary results demonstrated that the CBD ratio based approach could approximately represent the test results for $\mathrm{CO}_{2}, \mathrm{NOx}$ and fuel economy. As a result, correction factors were applied to $\mathrm{CO}_{2}$, $\mathrm{NOx}$ and fuel economy for those MY groups.

Correction factors for the DPF were determined for diesel buses, which could primarily be applied to older buses retrofitted with a DPF. Results showed that a DPF effectively reduced CO, HC and PM emissions on average by $86.4 \%, 95.8 \%$ and $92.2 \%$, respectively. The DPFs nearly did not affect the NOx emissions at all while on average they slightly reduced fuel economy by $2.6 \%$ as trade-offs.

\subsubsection{Comparison with EMFAC Model}

Diesel urban buses were selected to compare the IBIS emissions model and CARB's EMFAC model in terms of distance-specific emissions. For the EMFAC model, California statewide and Orange County were selected and the same results were observed for the two regions.

Comparison of results between the IBIS and EMFAC models agreed well for CO, NOx and PM for MY 2000 buses. For MY 2006 diesel buses, results agreed well for CO and roughly agreed for NOx and PM with higher values from the IBIS model. The EMFAC model output constant $\mathrm{CO}_{2}$ and fuel economy values across different speeds.

The EMFAC model showed constant values of emissions and fuel economy as vehicles age, which agreed with the IBIS model although IBIS did not account for deterioration impact.

For the EMFAC model, the trends as a function of model years demonstrated different technology groups as well as different emission standards adopted by the CARB. 


\subsection{Recommendations}

Some parameters have not be considered, including engine rated power, number of cylinders, engine displacement, transmission type, and heating system. The analysis of their impact on emissions and fuel economy could be performed if more test data became available. In addition, the impact of external operational conditions such as road grade, ambient temperature, altitudes, geographical location, and seasonal conditions could be investigated and implemented into the emissions model.

The selected cycle metrics other than average speed are still relatively difficult to obtain by transit agencies. It is suggested that the IBIS emissions model could help users determine cycle metrics as model inputs. The interface of the IBIS model could be updated with a few "what if" questions to the users. Based on the answers of these questions, the IBIS emissions model could approximately determine other cycle metrics.

Regarding the cycle and emissions generation, further experimental validation could be performed for additional virtual cycles and more repeat tests could be conducted to investigate the impact of microtrip history on HC and CO emissions.

Future research work could be conducted to investigate the dispersion impact. When continuous emissions data were prepared, dispersion effect was not considered while timealignment was performed. The dispersion of the signal could affect emissions rates [92].

More backbone models could be developed when newer emissions testing are conducted. For example, with more stringent emissions standards, more buses are equipped with the SCR to reduce NOx emissions. Backbone models could be developed to reflect the SCR impact. In addition, some transit agencies might have buses powered by alternative fuels, such as LNG, biofuel, etc. Alternative fuel powered buses do not share major market but emission models could be developed to cover a variety of transit bus fleets. 


\section{REFERENCES}

1. Frey, H.C., Unal, A., Rouphall, N.M., and Colyar, J.D., “On-Road Measurement of Vehicle Tailpipe Emissions Using a Portable Instrument,” Journal of the Air \& Waste Management Association, Vol. 53, pp. 992-1002, August 2003.

2. Kelly, N.A. and Groblicki, P.J., "Real-world Emissions from a Modern Production Vehicle Driven in Los Angeles,” Journal of the Air \& Waste Management Association, Vol. 43, pp. 1351-1357, July 1993.

3. Clark, N.N., Zhen, F., Wayne, W.S., Schiavone, J.J., Chambers, C., Golub, A.D., and Chandler, K.L., "Assessment of Hybrid-Electric Transit Bus Technology,” TCRP Report 132, Transportation Research Board, Washington, D.C., 2009.

4. Miller, C.A., Hidy, G., Hales, J., Kolb, C.E., Werner, A.S., Haneke, B., Parrish, D., Frey, C., Rojas-Blanco, L., DesLauriers, M., Pennell, B., and Mobley, J.D., "Air Emissions Inventories in North America: A Critical Assessment," Journal of the Air \& Waste Management Association, Vol. 56 (8), pp. 1115-1129, August 2006.

5. User's Guide to MOBILE6.1 and MOBILE6.2, U.S. Environmental Protection Agency, Technical Report, EPA420-R-03-010, 2003.

6. DeCorla-Souza, P., Everett, J., and Bernreuter, V., "Workshop on Transportation Air Quality Analysis: Participant's Notebook," Publication No. FHWA-141-94-011, September 1994.

7. National Research Council, "Expanding Metropolitan Highways: Implications for Air Quality and Energy Use - Special Report 245," Washington, D.C., 1995.

8. User's Guide to EMFAC2007, California Air Resources Board, November 2006.

9. EMFAC2011 Overview, California Air Resources Board, September 2011.

10. EMFAC2011 Technical Documentation, California Air Resources Board, September 2011.

11. User's Guide for the Final NONROAD2005 Model, EPA420-R-05-013, December 2005.

12. EPA's National Inventory Model (NMIM), A Consolidated Emissions Modeling System for MOBILE6 and NONROAD, EPA420-R-05-024, December 2005.

13. User's Guide for OFFROAD2007, California Air Resources Board, November 2007. 
14. MOVES2004 User Guide, EPA420-P-04-019, November 2004.

15. MOVES2009 User Guide, EPA-420-B-09-008, March 2009.

16. MOVES2010 User Guide, EPA-420-B-09-041, December 2009.

17. MOVE 2010a User Guide, EPA-420-B-10-036, August 2010.

18. MOVES2010b User Guide, EPA-420-B-12-001b, June 2012.

19. Nam, E.K., "Proof of Concept Investigation for the Physical Emission Rate Estimator (PERE) to be Used in MOVES,” Ford Research and Advanced Engineering, 2003.

20. Koupal, J., Cumberworth, M., Michaels, H., Beardsley, M., and Brzezinski, D., "Design and Implementation of MOVES: EPA's New Generation Mobile Source Emission Model," 12th International Emission Inventory Conference, San Diego, April 2003.

21. Motor Vehicle Emission Simulator Highway Vehicle Implementation (MOVES-HVI) Demonstration Version Software Design and Reference Manual Draft, EPA420-P-07-001 February 2007.

22. Technical Guidance on the Use of MOVES2010 for Emission Inventory Preparation in State Implementation Plans and Transportation Conformity, EPA-420-B-10-023, April 2010.

23. MOVES2004 Software Design Reference Manual: Draft, EPA420-P-04-017, November 2004.

24. Sonntag, D.B. and Gao, H.O., "The MOVES from MOBILE: Preliminary Comparisons of EPA’s Current and Future Mobile Emissions Models," Transportation Research Board 86th Annual Meeting, Washington, D.C., January 2007.

25. Hart, C., Koupal, J., and Giannelli, R., "EPA’s Onboard Analysis Shootout: Overview and Results," EPA420-R-02-026, October 2002.

26. Frey, H.C., Unal, A., Chen, J., Li, S., and Xuan, C., "Methodology for Developing Modal Emission Rates for EPA’s Multi-Scale Motor Vehicle \& Equipment Emission System," EPA420-R-02-027, Prepared by North Carolina State University for U.S. EPA, Ann Arbor, MI, August 2002.

27. Frey, H.C., Unal, A., Chen, J., and Li, S., "Evaluation and Recommendation of a Modal Method for Modeling Vehicle Emissions," Proceedings, U.S. Environmental Protection Agency Emission Inventory Conference, San Diego, CA, April 2003. 
28. Fomunung, I., Washington, S., Guensler R., and Bachman, W., "Validation of the MEASURE Automobile Emissions Model: A Statistical Analysis," Journal of Transportation Statistics, Vol. 3 (2), pp. 65-84, 2000.

29. Fomunung, I., Washington, S., and Guensler R., “A Statistical Model for Estimating Oxides of Nitrogen Emissions from Light-Duty Motor Vehicles,” Transportation Research D: Transport and Environment, Vol. 4, pp. 333-352, 1999.

30. Bachman, W., "A GIS-Based Modal Model of Automobile Exhaust Emissions - Final Report," Atlanta, GA, Prepared by Georgia Institute of Technology for U.S. Environmental Protection Agency, 1998.

31. Gajendran, P., "Development of a Heavy Duty Vehicle Emission Inventory Prediction Methodology,” Ph.D. Dissertation, West Virginia University, 2005.

32. Clark, N.N., Gajendran, P., and Kern, J.M., "A Predictive Tool for Emissions from HeavyDuty Diesel Vehicles," Environmental Science \& Technology 37 (1), pp. 7-15, 2003.

33. Barth, M., Scora, G., and Younglove, T., "Modal Emission Model for Heavy-Duty Diesel Vehicles,” Transportation Research Record: Journal of the Transportation Research Board, Vol. 1880, pp. 10-20, 2004.

34. An, F., Barth, M., Norbeck, J., and Ross, M., "Development of Comprehensive Modal Emissions Model Operating under Hot-Stabilized Conditions,” Transportation Research Record: Journal of the Transportation Research Board, Vol. 1587, pp. 52-62, 1997.

35. Hanzevack, E.L., Long, T.W., Atkinson, C.M., and Traver, M.L., "Virtual Sensors for Spark Ignition Engines Using Neural Networks,” Proceedings of the American Control Conference, Albuquerque, NM, Vol. 1, pp. 669-673, June 1997.

36. Desantes, J.M., López, J.J., García, J.M., and Hernández, L., “Application of Neural Networks for Prediction and Optimization of Exhaust Emissions in a H.D. Diesel Engine,” SAE 2002 World Congress, Detroit, MI, SAE Paper No. 2002-01-1144, March 2002.

37. Krijnsen, H.C., Van Kooten, W.J., Calis, H.A., Verbeek, R.P., and Van Den Bleek, C.M., "Prediction of NOx Emissions from a Transiently Operating Diesel Engine Using an Artificial Neural Network,” Chemistry Engineering Technology, Vol. 22, pp. 601-607, July 1999.

38. Tehranian, A., "Effects of Artificial Neural Networks Characterization on Prediction of Diesel Engine Emissions,” Master Thesis, West Virginia University, 2004. 
39. Thompson, G.J., Atkinson, C.M., Clark, N.N., Long, T.W., and Hanzevack, E., "Neural Network Modeling of the Emissions and Performance of a Heavy-Duty Diesel Engine,” Proceedings of the Institution of Mechanical Engineers, Part D: Journal of Automobile Engineering, Vol. 214 (2), pp. 111-126, 2000.

40. Bedick, C.R., “Optimization of a Retrofit Urea-SCR System,” Ph.D. Dissertation, West Virginia University, 2009.

41. Traver, M.L., Atkinson, R.J., and Atkinson, C.M., "Neural Network-Based Diesel Engine Emissions Prediction Using In-Cylinder Combustion Pressure," International Fuels \& Lubricants Meeting \& Exposition, Dearborn, MI, SAE Paper No. 1999-01-1532, May 1999.

42. Hashemi, N. and Clark, N.N., “Artificial Neural Network as a Predictive Tool for Emissions from Heavy-Duty Diesel Vehicles in Southern California,” International Journal of Engine Research, Vol. 8 (4), 2007.

43. Clark, N.N., Conley, J., Jarrett, R.P., Nennelli, A., and Tóth-Nagy, C., "Emissions Modeling of Heavy-Duty Conventional and Hybrid Electric Vehicles," International Fall Fuels and Lubricants Meeting and Exposition, San Antonio, TX, SAE Paper No. 2001-013675, September 2001.

44. Tóth-Nagy, C., Conley, J., Jarrett, R.P., and Clark, N.N, "Further Validation of Artificial Neural Network-Based Emissions Simulation Models for Conventional and Hybrid Electric Vehicles," Journal of the Air and Waste Management Association, Vol. 56, pp. 898-910, July 2006.

45. Perhinschi, M.G., Wayne, W.S., Clark, N.N., and Lyons, D.W., “Neural Network Modeling of Emissions from Medium-Duty Vehicles Operating on Fisher-Tropsch Synthetic Fuel,” SAE World Congress \& Exhibition, Detroit, MI, SAE Paper No. 2007-01-1080, April 2007.

46. Jarrett, R.P. and Clark, N.N., "Weighting of Parameters in Artificial Neural Network Prediction of Heavy-Duty Diesel Engine Emissions,” SAE Powertrain \& Fluid Systems Conference \& Exhibition, San Diego, CA, SAE Paper No. 2002-01-2878, October 2002.

47. Clark N.N., Tehranian, A., Jarrett, R.P., and Nine, R.D., “Translation of Distance-Specific Emissions Rates between Different Heavy Duty Vehicle Chassis Test Schedules,” Spring Fuels \& Lubricants Meeting \& Exhibition, Reno, NV, SAE Paper No. 2002-01-1754, May 2002. 
48. Markel, T., Brooker, A., Hendricks, T., Johnson, V., Kelly, K., Kramer, B., O’Keefe, V., Sprik, S., and Wipke, K., “ADVISOR: A Systems Analysis Tool for Advanced Vehicle Modeling,” Journal of Power Sources, Vol. 110 (2), pp. 255-266, August 2002.

49. Fuel Economy Measurement Test (Engineering Type) for Trucks and Buses, Society of Automotive Engineering Standards J1376, 1982 (Cancelled in 1997).

50. Emission Test Cycles: European Transient Cycle (ETC), http://www.dieselnet.com/ standards/cycles/etc.html, September 2007.

51. SAE J2711 Recommended Practice for Measuring Fuel Economy and Emissions of HybridElectric and Conventional Heavy-Duty Vehicle, Society of Automotive Engineers, Document No. J2711, 2002.

52. Wayne, W.S., Clark, N.N., Nine, R.D., and Elefante, D., “A Comparison of Emissions and Fuel Economy from Hybrid-Electric and Conventional-Drive Transit Buses,” Energy \& Fuels, Vol. 18, pp. 257-270, 2004.

53. Emission Test Cycles: The Braunschweig City Driving Cycle, http://www.dieselnet.com/ standards/cycles/braunschweig.html, September 2007.

54. Thompson, E.D., Ansari, M., and Eberhard, G.A., “A Truck and Bus Chassis Dynamometer Developed for Fuels and Lubricants Research,” International Fuels \& Lubricants Meeting \& Exposition, Tulsa, OK, SAE Technical Paper 902112, October 1990.

55. McKain, D.L., Clark, N.N., Balon, T.H., Moynihan, P.J., Lynch, S.A., and Webb, T.C., "Characterization of Emissions from Hybrid-Electric and Conventional Transit Buses," CEC/SAE Spring Fuels \& Lubricants Meeting \& Exposition, Paris, France, SAE Paper No. 2000-01-2011, June 2000.

56. Wayne, W.S., Clark, N.N., Nine, R.D., and Rosepiler, S., "Washington Metropolitan Area Transit Authority Diesel Emissions Control Retrofit Project,” Final Report, West Virginia University, Morgantown WV, September 2002.

57. Graboski, M.S., McCormick, R.L., Yanowitz, J., and Ryan, L., "Heavy-Duty Diesel Vehicle Testing for the Northern Front Range Air Quality Study," Fort Collins, CO, February 1998.

58. Nine, R.D., Clark, N.N., and Norton, P., "Effect of Emissions on Multiple Driving Test Schedules Performed on Two Heavy Duty-Vehicles," SAE Fall Fuels and Lubricants Meeting and Exposition, Baltimore, MD, SAE Paper No. 2000-01-2818, October 2000. 
59. Clark, N.N., Lyons, D.W., Bata, R.M., Gautam, M., Wang, W.G., Norton, P., and Chandler, K., "Natural Gas and Diesel Transit Bus Emissions: Review and Recent Data," SAE Truck and Bus Conference, Cleveland, OH, SAE Paper No. 973203, November 1997.

60. Code of Federal Regulations, Title 40, Part 86, Subpart N - Emission Regulations for New Otto-Cycle and Diesel Heavy-Duty Engines; Gaseous and Particulate Exhaust Test Procedures, Washington, D.C., 1998.

61. Heavy-Duty Vehicle Cycle Development. EPA Document EPA-PB-288-805, July 1978.

62. Schiavone, J., Wayne, W.S., Nine, R., and Clark, N.N., "Development of Bee-Line Transit Bus Dynamometer Driving Schedule,” Interim Task Report, Submitted to Westchester County Department of Transportation, October 2002.

63. O'Keefe, M.P, Simpson, A., Kelly, K.J., and Pedersen, D.S., "Duty Cycle Characterization and Evaluation Towards Heavy Hybrid Vehicle Applications," SAE World Congress \& Exhibition, Detroit, MI, SAE Paper No. 2007-01-0302, April 2007.

64. Clark, N.N., Zhen, F., Wayne, W.S., and Lyons, D.W., "Transit Bus Life Cycle Cost and Year 2007 Emissions Estimation," Final Report Prepared by West Virginia University for U.S. Federal Transit Administration, Washington, D.C., 2007.

65. Clark, N.N., Kern, J.M., Atkinson, C.M., and Nine, R.D., "Factors affecting heavy-duty diesel vehicle emissions," Journal of Air \& Waste Management Assoc., Vol. 52 (1), pp. 8494, January 2002.

66. Gautam, M., Clark, N.N., Riddle, W., Nine, R., Wayne, W.S., Maldonado, H., Agrawal, A., and Carlock, M., "Development and Initial Use of a Heavy-Duty Diesel Truck Test Schedule for Emissions Characterization," Journal of Fuels and Lubricants, Vol. 111, pp. 812-825, 2002.

67. Nine, R.D., Clark, N.N., Atkinson, C.M., and Daley, J.J., "Development of a Heavy Duty Chassis Dynamometer Driving Route," SAE Journal of Automobile Engineering, Proceeding Institution of Mechanical Engineers, Vol. 213, Part D, pp. 561-574, 1999.

68. Vora, K.A., Clark, N.N., Nine, R.D., Gautam, M., Wayne, W.S., Thompson, G.J., and Lyons, D.W., "Correlation Study of PM and NOx for Heavy-Duty Vehicles Across Multiple Drive Schedules," SAE Powertrain \& Fluid Systems Conference, Tampa, FL, SAE Paper No. 2004-01-3022, October 2004. 
69. Ahn, K., Rakha, H., Trani, A., and Van Aerde, M., "Estimating Vehicle Fuel Consumption and Emissions Based on Instantaneous Speed and Acceleration Levels," Journal of Transportation Engineering, Vol.128 (2), pp. 182-190, March/April 2002.

70. Rakha, H. and Ding, Y., "Impact of Stops on Vehicle Fuel Consumption," Journal of Transportation Engineering, Vol. 129 (1), pp. 23-32, 2003.

71. Wayne, W.S., Khan, ABM S., Clark, N.N., Lyons, D.W., Gautam, M., and Thompson, G., "Effect of Average Speed and Idle Duration on Exhaust Emissions from a Diesel Bus Tested on Fourteen Drive Cycles," Transportation Research Board Annual Meeting, Washington, D.C., Paper No. 07-2945, 2007,

72. Clark, N.N., Wayne, W.S., Khan, ABM S., Lyons, D.W., Gautam, M., McKain, D.L., Thompson, G., and Barnett, R.A., "Effects of Average Driving Cycle Speed on Lean-Burn Natural Gas Bus Emissions and Fuel Economy," SAE Fuels and Emissions Conference, Cape Town, SOAFR, SAE Paper No. 2007-01-0054, January 2007.

73. Khan, ABM S., Clark, N.N., Wayne, W.S., Gautam, M., Thompson, G., McKain, D.L., Lyons, D.W., and Barnett, R.A., "Weight Effect on Emissions and Fuel Consumption from Diesel and Lean-burn Natural Gas Transit Buses," Asia Pacific Automotive Engineering Conference, Hollywood, CA, SAE Paper No. 2007-01-3626, August 2007.

74. Andre, M. and Pronello, C., "Relative influence of acceleration and speed on emissions under actual driving conditions," International Journal of Vehicle Design, Vol. 18 (3-4), pp. 340-353, 1997.

75. Simpson, A., "Parametric Modeling of Energy Consumption in Road Vehicles," Ph.D. Thesis, School of Information Technology and Electrical Engineering, University of Queensland, Australia, February 2005.

76. Tu, J., Perhinschi, M.G., Wayne, W.S., Marlowe, C., Tamayo, S., and Clark, N.N., "Investigation of Integrated Bus Information System (IBIS) for Evaluation of Emissions and Fuel Economy of Transit Buses”, 21st CRC Real World Emissions Workshop, San Diego, CA, March 2011.

77. Integrated Bus Information System (IBIS), Center for Alternative Fuels Engines and Emissions (CAFEE), West Virginia University, ibis.wvu.edu, December 2012.

78. Marlowe, C.L., "Development of Computational Tools for Modeling Engine Fuel Economy and Emissions,” Master Thesis, West Virginia University, 2009. 
79. Code of Federal Regulations, Title 40, Part 1065 - Engine Testing Procedures, Washington, D.C., 2007.

80. Khan, ABM S., Clark, N.N., Thompson, G.J., Wayne, W.S., Gautam, M., Lyons, D.W., and Hawelti, D., "Idle Emissions from Heavy-Duty Diesel Vehicles - Review and Recent Data," Journal of the Air \& Waste Management Association, Vol. 56, pp. 1404-1419, October 2006.

81. Wang, W.G., Palmer, G.N., Bata, R.M., Clark, N.N., Gautam, M., and Lyons, D.W., "Determination of Heavy-Duty Vehicle Energy Consumption by a Chassis Dynamometer," SAE Transactions, Journal of Commercial Vehicles, Vol. 101, pp. 687-696, 1992.

82. Clark, N.N., Gautam, M., Bata, R.M., Loth, J., Palmer, G.M., Wang, W.G., and Lyons, D.W., "Design and Operation of a New Transportable Laboratory for Emissions Testing of Heavy-duty Trucks and Buses,” International Journal of Vehicle Design (Heavy Vehicle Systems) Vol. 2, No. 3/4, pp. 285-299, 1995.

83. Center for Alternative, Engine and Emissions (CAFEE), West Virginia University, http://cafee.wvu.edu/about, December 2011.

84. Tu, J., Wayne, W.S., and Perhinschi, M.G., "Correlation Analysis of Duty Cycle Effects on Exhaust Emissions and Fuel Economy”, accepted for publication in the Journal of the Transportation Research Forum, 2012.

85. Freund, R.J., Littell, R.C., and Creighton, L., Regression Using JMP, SAS Publishing, June 11, 2003.

86. MATLAB Data Analysis, The Math Workds, Inc., March 2006.

87. Kahaner, D., Moler, C., and Nash, S., Numerical Methods and Software, Prentice Hall, 1988.

88. Boriboonsomsin, K. and Uddin, W., "Simplified Methodology to Estimate Emissions from Mobile Sources for Ambient Air Quality Assessment," Journal of Transportation Engineering, Vol. 132 (10), pp. 817-828, 2006.

89. Wang, W.G., Lyons, D.W., Clark, N.N., Gautam, M., Norton, P.M., "Emissions from Nine Heavy Trucks Fueled by Diesel and Biodiesel Blend without Engine Modification," Environmental Science \& Technology, Vol. 34, pp. 933-939, 2000. 
90. Perhinschi, M.G., Marlowe, C., Tamayo, S., Tu, J., and Wayne, W.S., "Evolutionary Algorithm for Vehicle Driving Cycle Generation”, Journal of Air and Waste Management Association, Vol. 61, pp. 923-931, 2011.

91. Tu, J., Wayne, W.S., Perhinschi, M.G., Marlowe, C., Tamayo, S., and Clark, N.N., “Development of Duty Cycle Generator Based on Genetic Algorithm for Emission and Fuel Economy Modeling”, 19th CRC On-Road Vehicle Emissions Workshop, San Diego, CA, March 2009.

92. Madireddy, M.R., "Methods for Reconstruction of Transient Emissions from Heavy-Duty Vehicles,” Ph.D. Dissertation, West Virginia University, 2008.

93. Cummins Inc., “Better Every ${ }^{\mathrm{TM}}$ Route - For Urban Transit Application,” Bulletin 4971254, September 2011.

94. Pang, S.H, "Life Cycle Inventory Incorporating Fuel Cycle and Real-world In-use Measurement Data for Construction Equipment and Vehicles," Ph.D. Dissertation, North Carolina State University, 2008.

95. Chiu J.P., Wegrzin J., and Muphy K.E., “Low Emissions Class 8 Heavy-Duty, On-Highway Natural Gas and Gasoline Engine,” 2004 Powertrain \& Fluid Systems Conference \& Exhibition, Tampa, FL, SAE Paper No. 2004-01-2982, October 2004.

96. Einewall P., Tunestal P., and Johansson B., "Lean-burn Natural Gas Operation vs. Stoichiometric Operation with EGR and a Three-Way Catalyst,” SAE 2005 World Congress \& Exhibition, Detroit, MI, SAE Paper No. 2005-01-0250, April 2005.

97. Nylund N., Erkkil K., Lappi M., and Ikonen M., "Transit Bus Emission Study: Comparison of Emissions from Diesel and Natural Gas Buses,” Research Report, Technical Research Centre of Finland (VTT), October 2004.

98. Messer, J.T., Clark, N.N., and Lyons, D.W., "Measurement Delays and Modal Analysis for a Heavy-Duty Transportable Laboratory,” Society of Automotive Engineers, Warrendale, PA, SAE 950218, 1995.

99. Zhang, Y., "Experimental and Numerical Study of the Behavior of Three-Way Catalytic Converters under Different Engine Operation Conditions,” Ph.D. Dissertation, Massachusetts Institute of Technology, 2005.

100. Ehsani, M., Gao, Y., Gay, S.E., and Emadi, A., Modern Electric, Hybrid Electric, and Fuel Cell Vehicles: Fundamentals, Theory, and Design, CRC Press, 2004. 
101. Regulatory Announcement, "Heavy-Duty Engine and Vehicle Standards and Highway Diesel Fuel Sulfur Control Requirements,” U.S. Environmental Protection Agency, EPA420-F-00-057, December 2000.

102. Technical Bulletin, "Diesel Particulate Filter General Information," U.S. Environmental Protection Agency, EPA-420-F-10-029, May 2010.

103. Wayne, W.S., Perhinschi, M.G., Clark, N.N., Tamayo, S., and Tu, J., "Integrated Bus Information System (IBIS) - A Vehicle Procurement Resource for Transit,” Transportation Research Record: Journal of the Transportation Research Board, Vol. 2233, pp. 1-10, 2011.

104. Conversion Factors for Hydrocarbon Emission Components, EPA420-P-03-002, May 2003.

105. California Adopts New Emission Standards for Urban Buses, http://www.dieselnet.com/ news/2000/02carb.php, January 2013. 


\section{APPENDIX A - WVU EMISSION DATABASE}

Table A-1 A WVU Short Report of Emission Test

Test Sequence Number: 4482

WVU Test Reference Number: WMATA-2094-ULSD1-Man1

Fleet Owner Full Name

Fleet Address

Fleet Address (City, State, Zip)

Vehicle Type

Vehicle ID Number (VIN)

Vehicle Manufacturer

Vehicle Model Year

Gross Vehicle Weight (GVW) (Ib.)

Vehicle Total Curb Weight (Ib.)

Vehicle Tested Weight (lb.)

Odometer Reading (mile)

Transmission Type

Transmission Configuration

Number of Axles

Engine Type

Engine ID Number

Engine Model Year

Engine Displacement (Liter)

Number of Cylinders

Engine Rated Power (hp)

Primary Fuel

Test Cycle

Test Date

Engineer

Driver

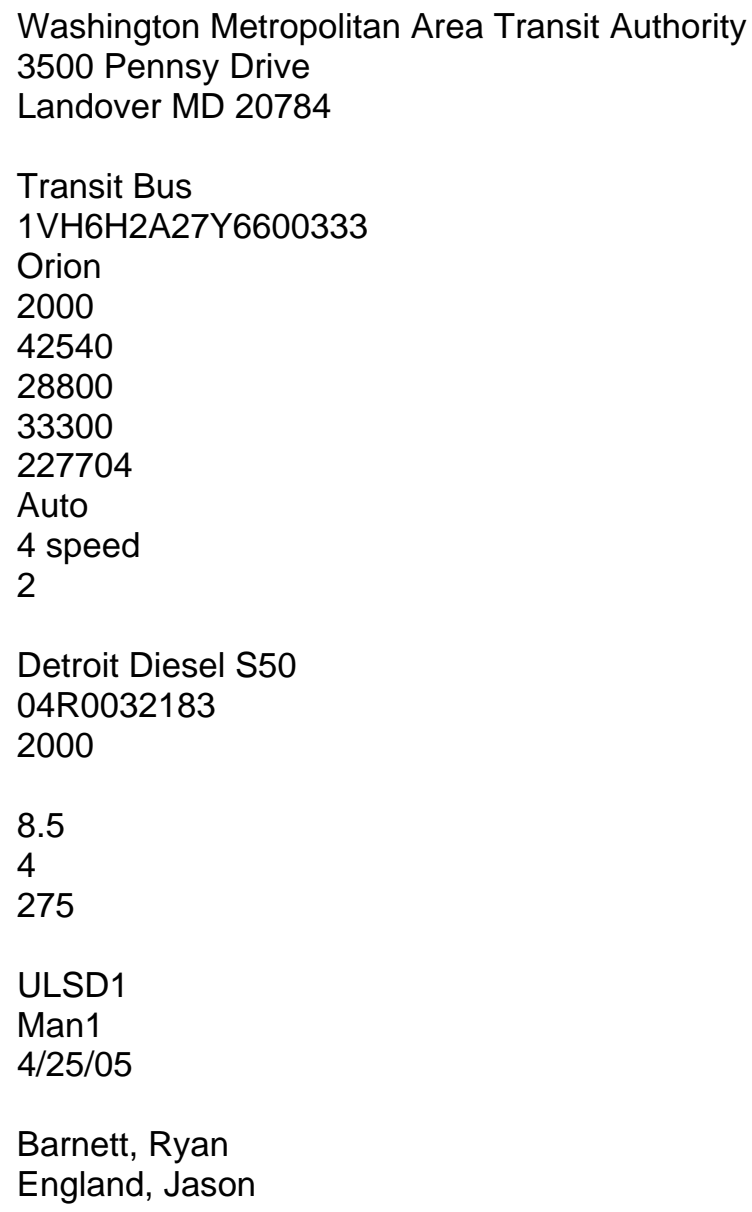

Emissions Results (g/mile)

Fuel Economy

\begin{tabular}{|c|c|c|c|c|c|c|c|c|c|}
\hline Run Seq. No. & $\mathrm{CO}$ & $\mathrm{NO}_{x}{ }^{1}$ & $\mathrm{NO}_{x}{ }^{2}$ & FIDHC & PM & $\mathrm{CO}_{2}$ & mile/gal & BTU/mile & Miles \\
\hline $4482-1$ & 6.22 & 27.9 & 27.8 & 0.19 & 0.57 & 3529 & 2.73 & 46876 & 2.12 \\
\hline $4482-2$ & 6.83 & 27.7 & 26.9 & 0.22 & 0.55 & 3568 & 2.70 & 47414 & 2.09 \\
\hline 4482 Average & 6.53 & 27.8 & 27.4 & 0.20 & 0.56 & 3548 & 2.71 & 47145 & 2.11 \\
\hline Std. Dev. & 0.43 & 0.1 & 0.7 & 0.02 & 0.01 & 28 & 0.02 & 381 & 0.02 \\
\hline CV\% & 6.6 & 0.5 & & 9.3 & 2.6 & 0.8 & 0.8 & 0.8 & 0.8 \\
\hline
\end{tabular}

x-Not Reportable, a-Outlier, b-HC Not Reportable(Residual HC), c-missing component, d-Coefficient of Variation Too Large, e-below detectable limit Test Purpose:

Testing of WMATA bus \#2094 for cycle comparison purposes

Special Procedures:

run 2 is $\mathrm{NO} / \mathrm{NOx}$ split 
Figure A-2 Screenshot of a Query Example of WVU MS Access Database - Integrated Data for Transit Buses

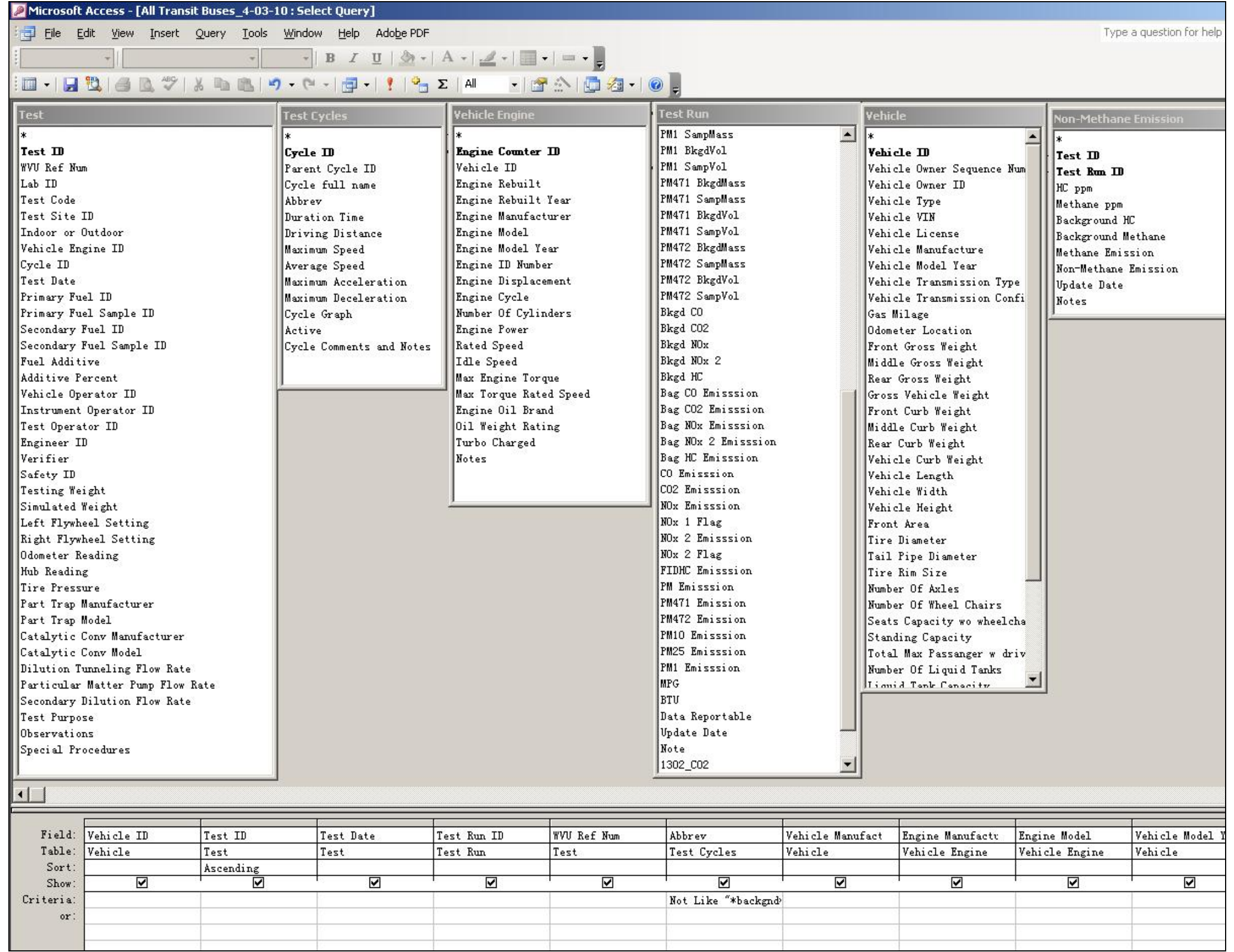


Table A-3 An Example of WVU Database - Portion of Query Results of Integrated Data for Transit Buses

\begin{tabular}{|c|c|c|c|c|c|c|c|c|}
\hline Test Date & Test Run ID & WVU Ref Num & Abbrev & Vehicle Manufacture & Engine Manufacturer & Engine Model & Vehicle Model Year & Odometer Reading \\
\hline $5 / 18 / 2006$ & 1 & WMATA-2639-CNG-NYBUS & NYBus & Orion & John Deere & $6081 \mathrm{H}$ & 2005 & 4225 \\
\hline $5 / 18 / 2006$ & 2 & WMATA-2639-CNG-NYBUS & NYBus & Orion & John Deere & $6081 \mathrm{H}$ & 2005 & 4225 \\
\hline $5 / 18 / 2006$ & 1 & WMATA-2639-CNG-MAN & Manhattan & Orion & John Deere & $6081 \mathrm{H}$ & 2005 & 4225 \\
\hline $5 / 18 / 2006$ & 1 & WMATA-2639-CNG-BRAUN & BRAUN & Orion & John Deere & $6081 \mathrm{H}$ & 2005 & 4225 \\
\hline $5 / 19 / 2006$ & 1 & WMATA-2639-CNG-NYBUS & NYBus & Orion & John Deere & $6081 \mathrm{H}$ & 2005 & 4225 \\
\hline $5 / 19 / 2006$ & 1 & WMATA-2639-CNG-OCTA & OCTA & Orion & John Deere & $6081 \mathrm{H}$ & 2005 & 4225 \\
\hline $5 / 19 / 2006$ & 1 & WMATA-2639-CNG-PARIS & PARIS & Orion & John Deere & $6081 \mathrm{H}$ & 2005 & 4225 \\
\hline $5 / 19 / 2006$ & 1 & WMATA-2639-CNG-WMATA & WMATA & Orion & John Deere & $6081 \mathrm{H}$ & 2005 & 4225 \\
\hline $6 / 14 / 2006$ & 2 & WMATA-6001-ULSD1-NYBUS & NYBus & New Flyer & Cummins & ISL280 & 2006 & 18551 \\
\hline $6 / 14 / 2006$ & 1 & WMATA-6001-ULSD1-PARIS & PARIS & New Flyer & Cummins & ISL280 & 2006 & 18551 \\
\hline $6 / 14 / 2006$ & 1 & WMATA-6001-ULSD1-OCTA & OCTA & New Flyer & Cummins & ISL280 & 2006 & 18551 \\
\hline $6 / 14 / 2006$ & 2 & WMATA-6001-ULSD1-OCTA & OCTA & New Flyer & Cummins & ISL280 & 2006 & 18551 \\
\hline $6 / 14 / 2006$ & 1 & WMATA-6001-ULSD1-BRAUN & BRAUN & New Flyer & Cummins & ISL280 & 2006 & 18551 \\
\hline $6 / 14 / 2006$ & 1 & WMATA-6001-ULSD1-MAN & Manhattan & New Flyer & Cummins & ISL280 & 2006 & 18551 \\
\hline $6 / 15 / 2006$ & 2 & WMATA-6001-ULSD1-WMATA & WMATA & New Flyer & Cummins & ISL280 & 2006 & 18551 \\
\hline $6 / 30 / 2006$ & 1 & WMATA-6001-ULSD1-NYBUS & NYBus & New Flyer & Cummins & ISL280 & 2006 & 18551 \\
\hline $6 / 30 / 2006$ & 2 & WMATA-6001-ULSD1-NYBUS & NYBus & New Flyer & Cummins & ISL280 & 2006 & 18551 \\
\hline $6 / 30 / 2006$ & 1 & WMATA-6001-ULSD1-MAN & Manhattan & New Flyer & Cummins & ISL280 & 2006 & 18551 \\
\hline $6 / 30 / 2006$ & 1 & WMATA-6001-ULSD1-BRAUN & BRAUN & New Flyer & Cummins & ISL280 & 2006 & 18551 \\
\hline $7 / 5 / 2006$ & 1 & WMATA-6001-ULSD1-NYBUS & NYBus & New Flyer & Cummins & ISL280 & 2006 & 18551 \\
\hline $7 / 5 / 2006$ & 2 & WMATA-6001-ULSD1-OCTA & OCTA & New Flyer & Cummins & ISL280 & 2006 & 18551 \\
\hline $7 / 5 / 2006$ & 1 & WMATA-6001-ULSD1-OCTA & OCTA & New Flyer & Cummins & ISL280 & 2006 & 18551 \\
\hline $7 / 6 / 2006$ & 2 & WMATA-6001-ULSD1-WMATA & WMATA & New Flyer & Cummins & ISL280 & 2006 & 18551 \\
\hline $7 / 6 / 2006$ & 1 & WMATA-6001-ULSD1-WMATA & WMATA & New Flyer & Cummins & ISL280 & 2006 & 18551 \\
\hline $7 / 6 / 2006$ & 1 & WMATA-6001-ULSD1-PARIS & PARIS & New Flyer & Cummins & ISL280 & 2006 & 18551 \\
\hline $5 / 23 / 2006$ & 1 & WMATA-2621-CNG-NYBUS & NYBus & Orion & John Deere & $6081 \mathrm{H}$ & 2005 & 3148 \\
\hline $5 / 23 / 2006$ & 2 & WMATA-2621-CNG-NYBUS & NYBus & Orion & John Deere & $6081 \mathrm{H}$ & 2005 & 3148 \\
\hline $5 / 23 / 2006$ & 3 & WMATA-2621-CNG-NYBUS & NYBus & Orion & John Deere & $6081 \mathrm{H}$ & 2005 & 3148 \\
\hline
\end{tabular}

To be continued at next page (additional columns) 


\begin{tabular}{|c|c|c|c|c|c|c|c|c|c|c|c|c|c|}
\hline Test Date & $\begin{array}{l}\text { Catalytic Conv } \\
\text { Manufacturer }\end{array}$ & $\begin{array}{c}\text { Part Trap } \\
\text { Manufacturer }\end{array}$ & $\begin{array}{l}\text { Primary } \\
\text { Fuel ID }\end{array}$ & $\begin{array}{l}\text { Testing } \\
\text { Weight }\end{array}$ & $\begin{array}{l}\mathrm{CO} \\
\mathrm{g} / \mathrm{mile}\end{array}$ & $\begin{array}{l}\text { NOx } \\
\text { g/mile }\end{array}$ & $\begin{array}{l}\mathrm{HC} \\
\mathrm{g} / \mathrm{mile}\end{array}$ & $\begin{array}{l}\text { PM } \\
\text { g/mile }\end{array}$ & $\begin{array}{l}\mathrm{CO} 2 \\
\mathrm{~g} / \mathrm{mile}\end{array}$ & MPG & $\begin{array}{l}\text { Ave } \\
\text { Speed }\end{array}$ & $\begin{array}{l}\text { Duration } \\
\text { Time }\end{array}$ & $\begin{array}{c}\text { Actual } \\
\text { Driving } \\
\text { Distance }\end{array}$ \\
\hline $5 / 18 / 2006$ & $\begin{array}{l}\text { Oxidation } \\
\text { Catalyst }\end{array}$ & & CNG & 36700 & 0.09 & 29.07 & 47.17 & 0.20 & 5207.83 & 1.31 & 3.38 & 600 & 0.56 \\
\hline $5 / 18 / 2006$ & $\begin{array}{l}\text { Oxidation } \\
\text { Catalyst }\end{array}$ & & CNG & 36700 & 0.45 & 32.67 & 55.11 & 0.09 & 5156.30 & 1.31 & 3.32 & 600 & 0.55 \\
\hline $5 / 18 / 2006$ & $\begin{array}{l}\text { Oxidation } \\
\text { Catalyst }\end{array}$ & & CNG & 36700 & 0.12 & 18.48 & 25.07 & 0.02 & 2972.05 & 2.29 & 6.68 & 1089.1 & 2.02 \\
\hline $5 / 18 / 2006$ & $\begin{array}{l}\text { Oxidation } \\
\text { Catalyst }\end{array}$ & & CNG & 36700 & 0.09 & 13.07 & 13.45 & 0.02 & 1747.78 & 3.91 & & 0 & 6.66 \\
\hline $5 / 19 / 2006$ & $\begin{array}{l}\text { Oxidation } \\
\text { Catalyst }\end{array}$ & & CNG & 36700 & -0.27 & 38.08 & 69.51 & 0.06 & 5190.70 & 1.30 & 3.41 & 600 & 0.57 \\
\hline $5 / 19 / 2006$ & $\begin{array}{l}\text { Oxidation } \\
\text { Catalyst }\end{array}$ & & CNG & 36700 & -0.02 & 10.99 & 12.13 & 0.01 & 1822.57 & 3.76 & 12.20 & 1950 & 6.61 \\
\hline $5 / 19 / 2006$ & $\begin{array}{l}\text { Oxidation } \\
\text { Catalyst }\end{array}$ & & CNG & 36700 & 0.02 & 20.16 & 23.75 & 0.02 & 2793.59 & 2.44 & & 0 & 3.46 \\
\hline 5/19/2006 & $\begin{array}{l}\text { Oxidation } \\
\text { Catalyst }\end{array}$ & & CNG & 36700 & -0.03 & 11.62 & 13.55 & 0.01 & 2265.87 & 3.03 & 8.44 & 1839 & 4.31 \\
\hline $6 / 14 / 2006$ & & Engelhard & ULSD1 & 35860 & 0.19 & 12.32 & 0.06 & 0.02 & 2652.57 & 3.64 & & 0 & 3.47 \\
\hline $6 / 14 / 2006$ & & Engelhard & ULSD1 & 35860 & 0.03 & 8.16 & 0.02 & 0.03 & 1843.61 & 5.24 & 12.15 & 1950 & 6.58 \\
\hline $6 / 14 / 2006$ & & Engelhard & ULSD1 & 35860 & 0.02 & 7.81 & 0.01 & 0.04 & 1799.12 & 5.37 & 12.13 & 1950 & 6.57 \\
\hline $6 / 14 / 2006$ & & Engelhard & ULSD1 & 35860 & 0.01 & 7.40 & -0.03 & 0.05 & 1805.98 & 5.35 & & 0 & 6.72 \\
\hline $6 / 14 / 2006$ & & Engelhard & ULSD1 & 35860 & -0.01 & 11.45 & 0.01 & 0.03 & 2654.43 & 3.64 & 6.75 & 1089.1 & 2.04 \\
\hline $6 / 15 / 2006$ & & Engelhard & ULSD1 & 35860 & 0.19 & 11.18 & 0.03 & 0.02 & 2311.45 & 4.18 & 8.31 & 1839 & 4.24 \\
\hline $6 / 15 / 2006$ & & Engelhard & ULSD1 & 35860 & 0.19 & 10.45 & 0.01 & 0.03 & 2236.53 & 4.32 & 8.34 & 1839 & 4.26 \\
\hline $6 / 30 / 2006$ & & Engelhard & ULSD1 & 35860 & -0.05 & 17.72 & -0.02 & 0.07 & 3915.27 & 2.47 & 3.59 & 600 & 0.60 \\
\hline $6 / 30 / 2006$ & & Engelhard & ULSD1 & 35860 & 0.37 & 17.12 & 0.25 & 0.04 & 3883.00 & 2.49 & 3.57 & 600 & 0.60 \\
\hline $6 / 30 / 2006$ & & Engelhard & ULSD1 & 35860 & 0.12 & 11.30 & 0.19 & 0.00 & 2454.07 & 3.93 & 6.81 & 1089.1 & 2.06 \\
\hline $6 / 30 / 2006$ & & Engelhard & ULSD1 & 35860 & 0.02 & 8.10 & 0.06 & 0.01 & 1778.66 & 5.43 & & 0 & 6.69 \\
\hline $7 / 5 / 2006$ & & Engelhard & ULSD1 & 35860 & -0.06 & 19.17 & 0.07 & 0.10 & 4259.44 & 2.27 & 3.66 & 600 & 0.61 \\
\hline $7 / 5 / 2006$ & & Engelhard & ULSD1 & 35860 & 0.08 & 8.22 & 0.01 & 0.04 & 1820.90 & 5.30 & 12.12 & 1950 & 6.57 \\
\hline $7 / 5 / 2006$ & & Engelhard & ULSD1 & 35860 & 0.06 & 8.36 & -0.01 & 0.05 & 1850.95 & 5.22 & 12.19 & 1950 & 6.60 \\
\hline $7 / 6 / 2006$ & & Engelhard & ULSD1 & 35860 & 0.02 & 9.97 & 0.04 & 0.02 & 2182.30 & 4.42 & 8.28 & 1839 & 4.23 \\
\hline $7 / 6 / 2006$ & & Engelhard & ULSD1 & 35860 & 0.09 & 11.04 & 0.19 & 0.02 & 2318.40 & 4.16 & & 0 & 3.52 \\
\hline $5 / 23 / 2006$ & $\begin{array}{l}\text { Oxidation } \\
\text { Catalyst }\end{array}$ & & CNG & 36700 & 0.11 & 31.36 & 56.59 & 0.13 & 4717.90 & 1.43 & 3.84 & 600 & 0.64 \\
\hline $5 / 23 / 2006$ & $\begin{array}{l}\text { Oxidation } \\
\text { Catalyst }\end{array}$ & & CNG & 36700 & 0.81 & 50.61 & 51.03 & 0.06 & 4980.78 & 1.36 & 3.65 & 600 & 0.61 \\
\hline $5 / 23 / 2006$ & $\begin{array}{l}\text { Oxidation } \\
\text { Catalyst }\end{array}$ & & CNG & 36700 & 0.05 & 50.52 & 56.77 & 0.13 & 4861.21 & 1.39 & 3.67 & 600 & 0.61 \\
\hline
\end{tabular}




\section{APPENDIX B - EMISSIONS TESTING OF VIRTUAL CYCLES}

\section{B.1 CNG Bus}
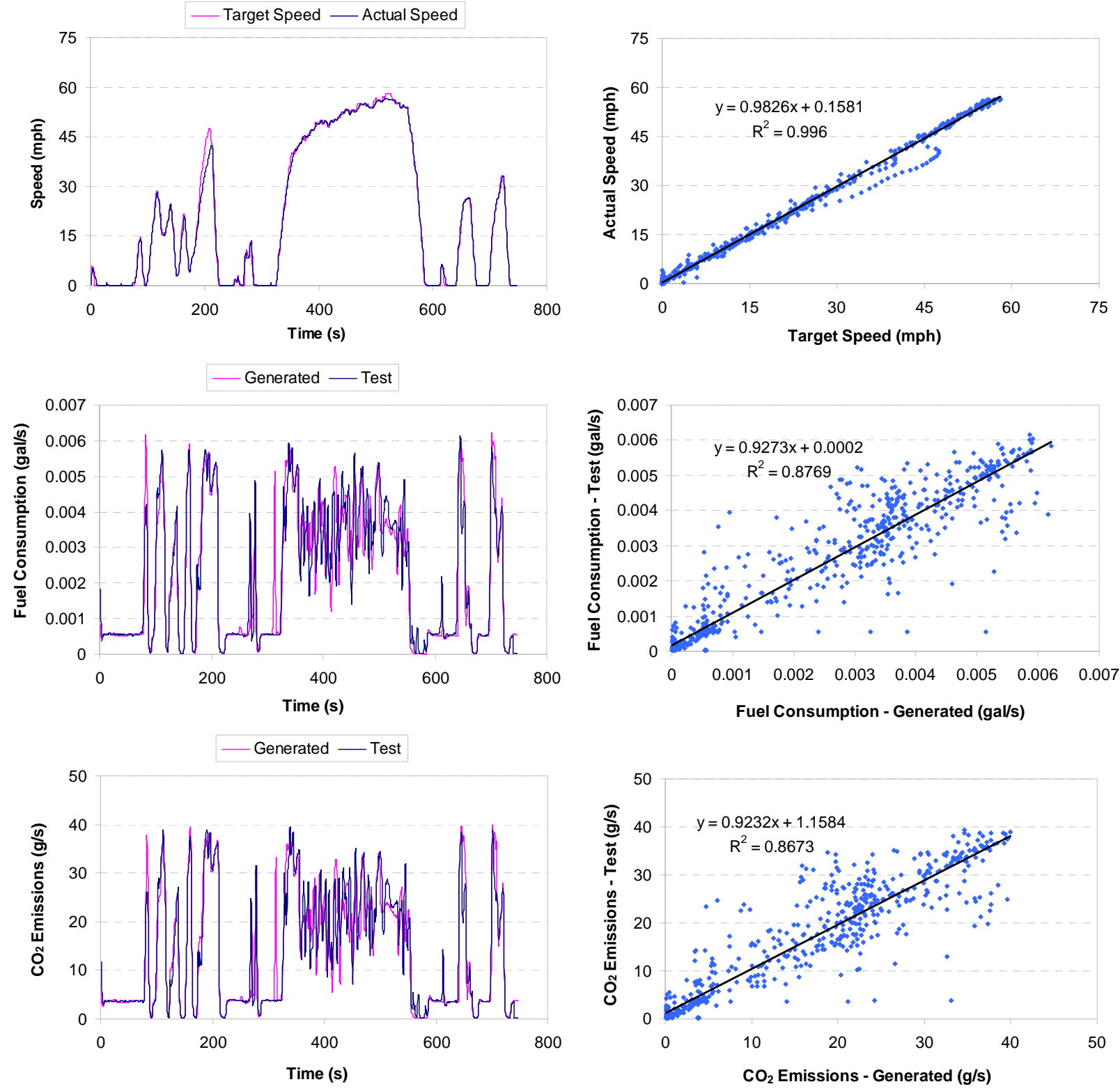

Figure B-1 Target and Actual Vehicle Speed, Fuel Consumption and $\mathrm{CO}_{2}$ Rates from Emissions Testing and Cycle Generation of the VC_274 from a MY 2008 CNG Bus 

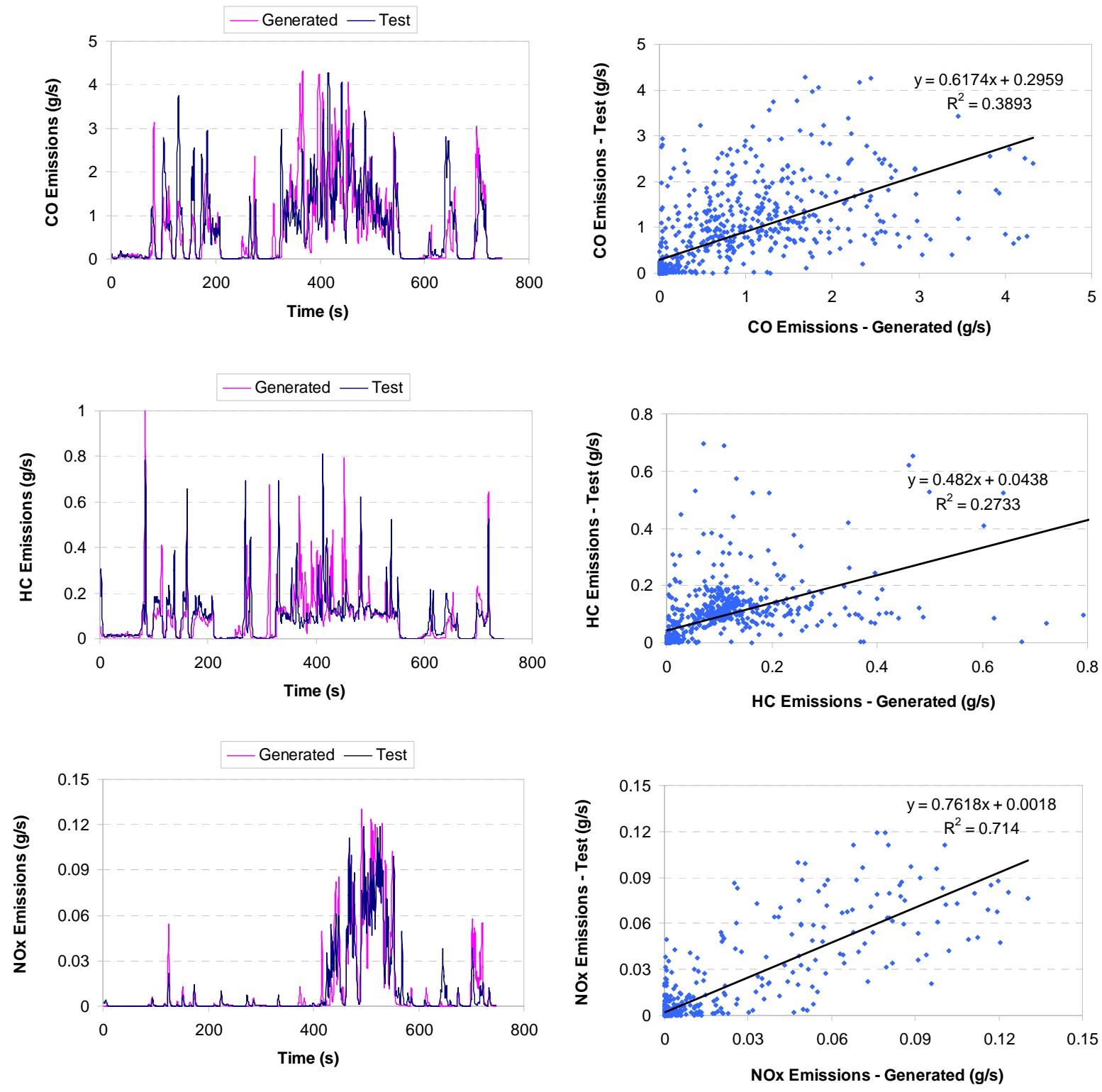

Figure B-2 CO, HC and NOx Rates from Emissions Testing and Cycle Generation of the VC_274 from a MY 2008 CNG Bus 

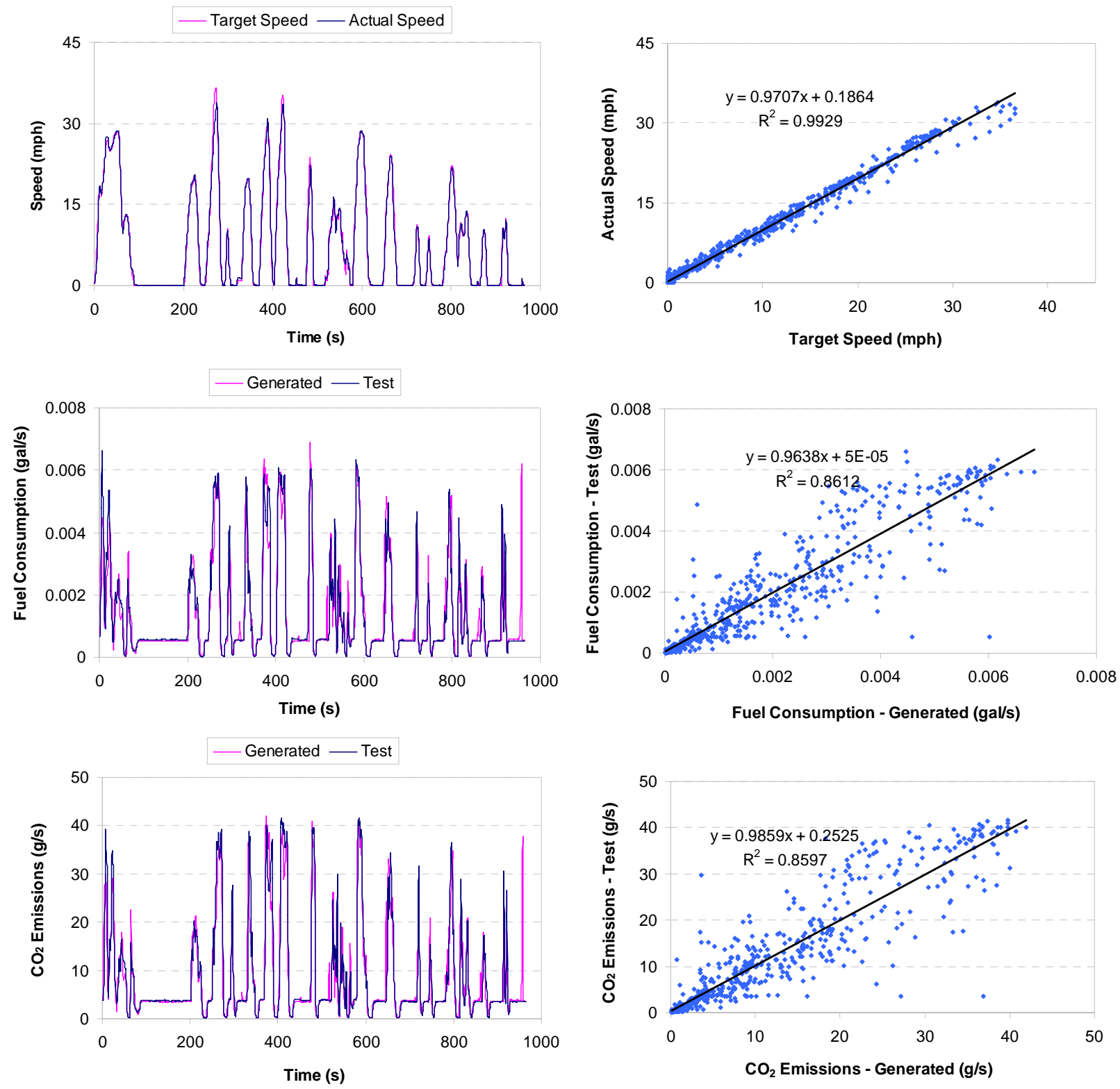

Figure B-3 Target and Actual Vehicle Speed, Fuel Consumption and $\mathrm{CO}_{2}$ Rates from Emissions Testing and Cycle Generation of the VC_28 from a MY 2008 CNG Bus 

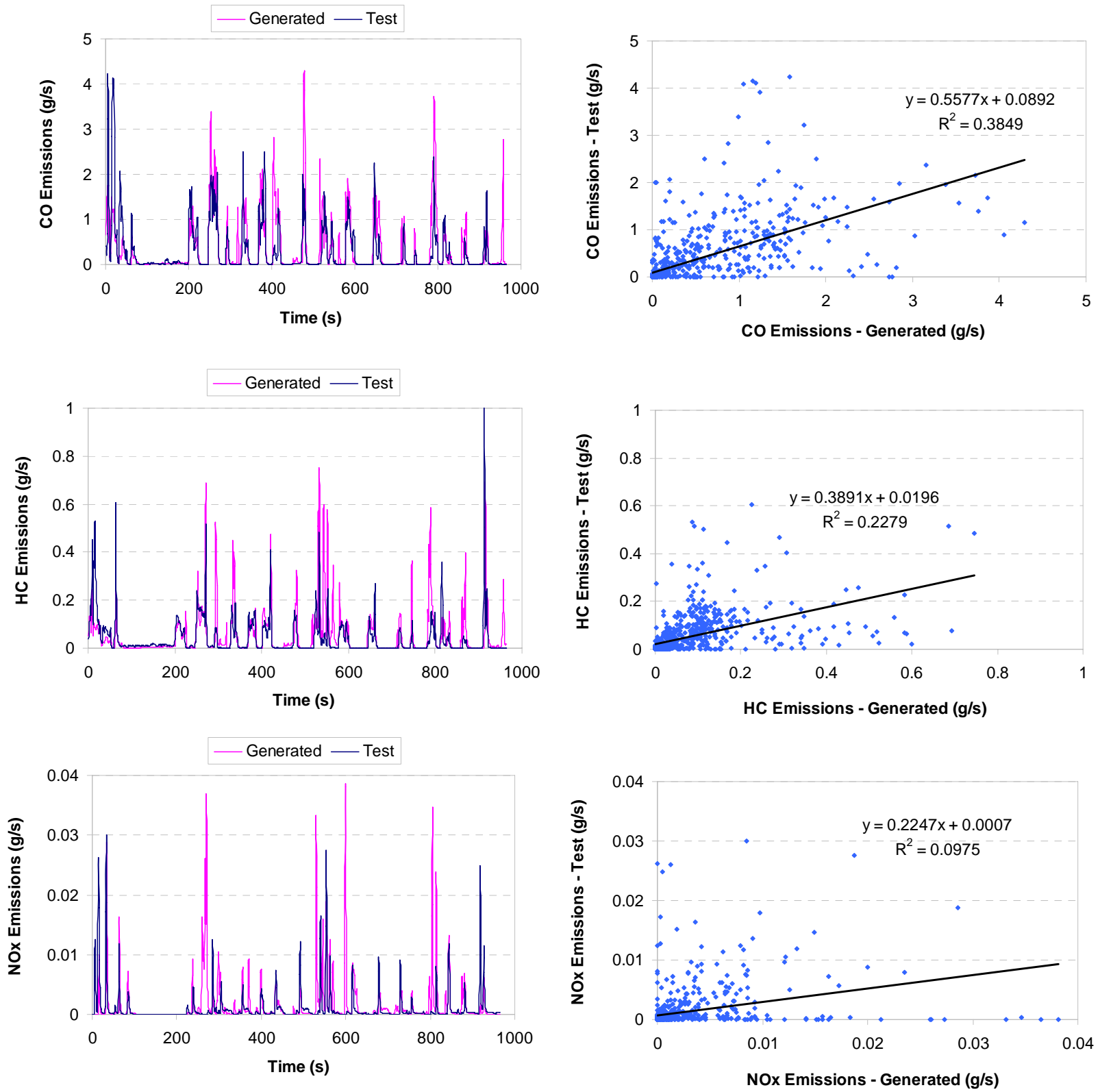

Figure B-4 CO, HC and NOx Rates from Emissions Testing and Cycle Generation of the VC_28 from a MY 2008 CNG Bus 


\section{B.2 Hybrid Bus}
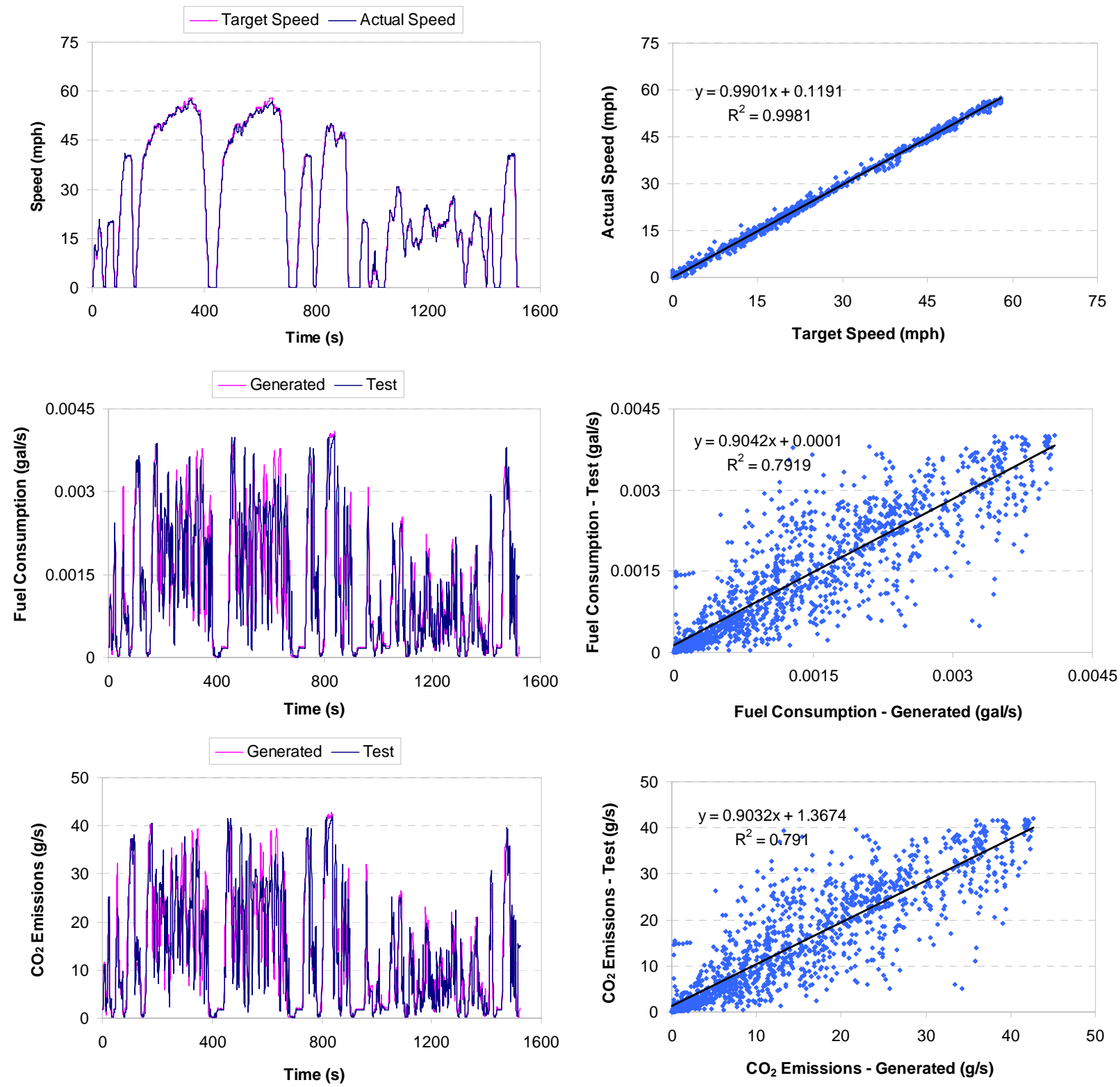

Figure B-5 Target and Actual Vehicle Speed, Fuel Consumption and $\mathrm{CO}_{2}$ Rates from Emissions Testing and Cycle Generation of the VC_637 from a MY 2008 Hybrid Bus 

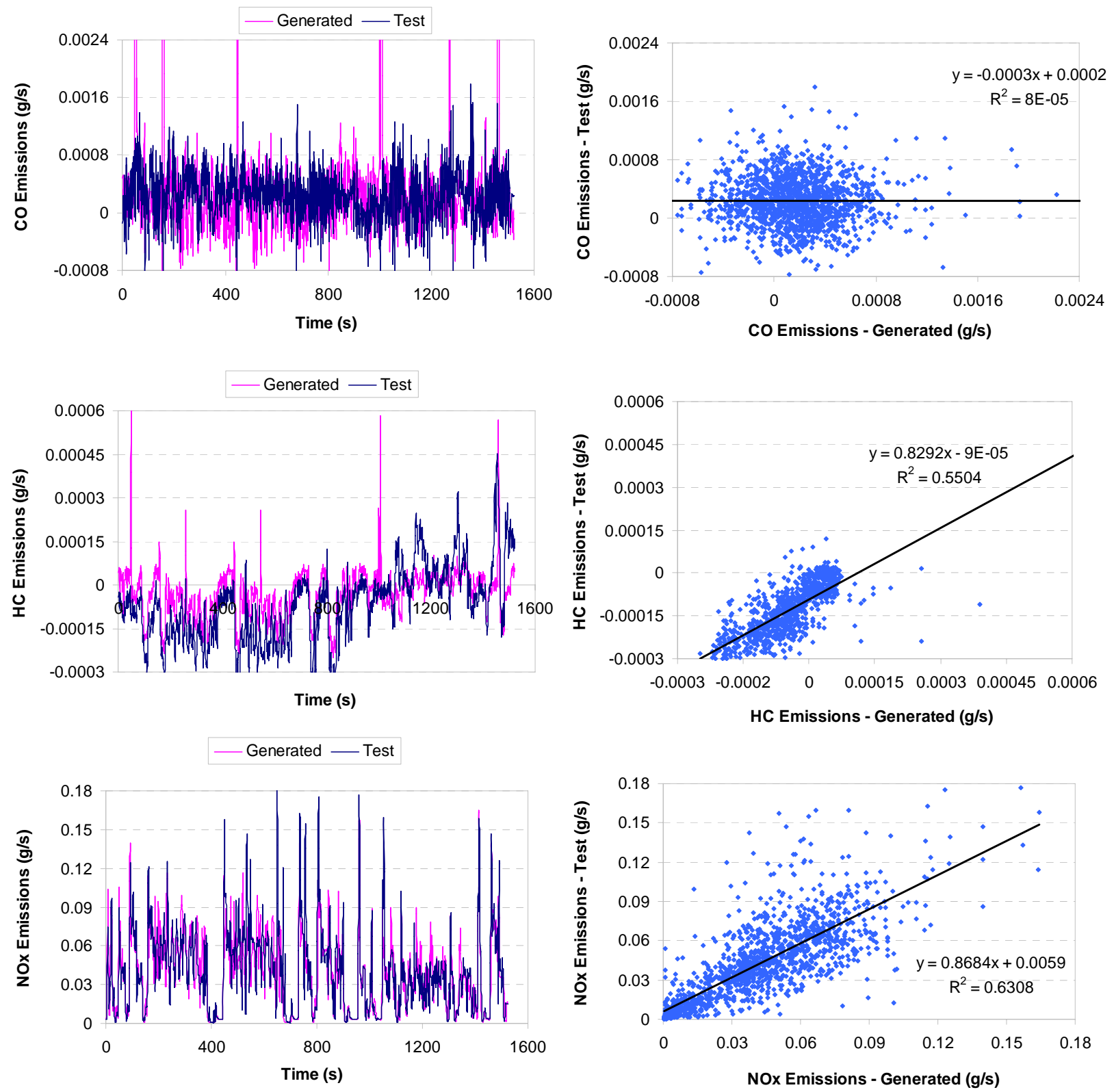

Figure B-6 CO, HC and NOx Rates from Emissions Testing and Cycle Generation of the VC_637 from a MY 2008 Hybrid Bus 

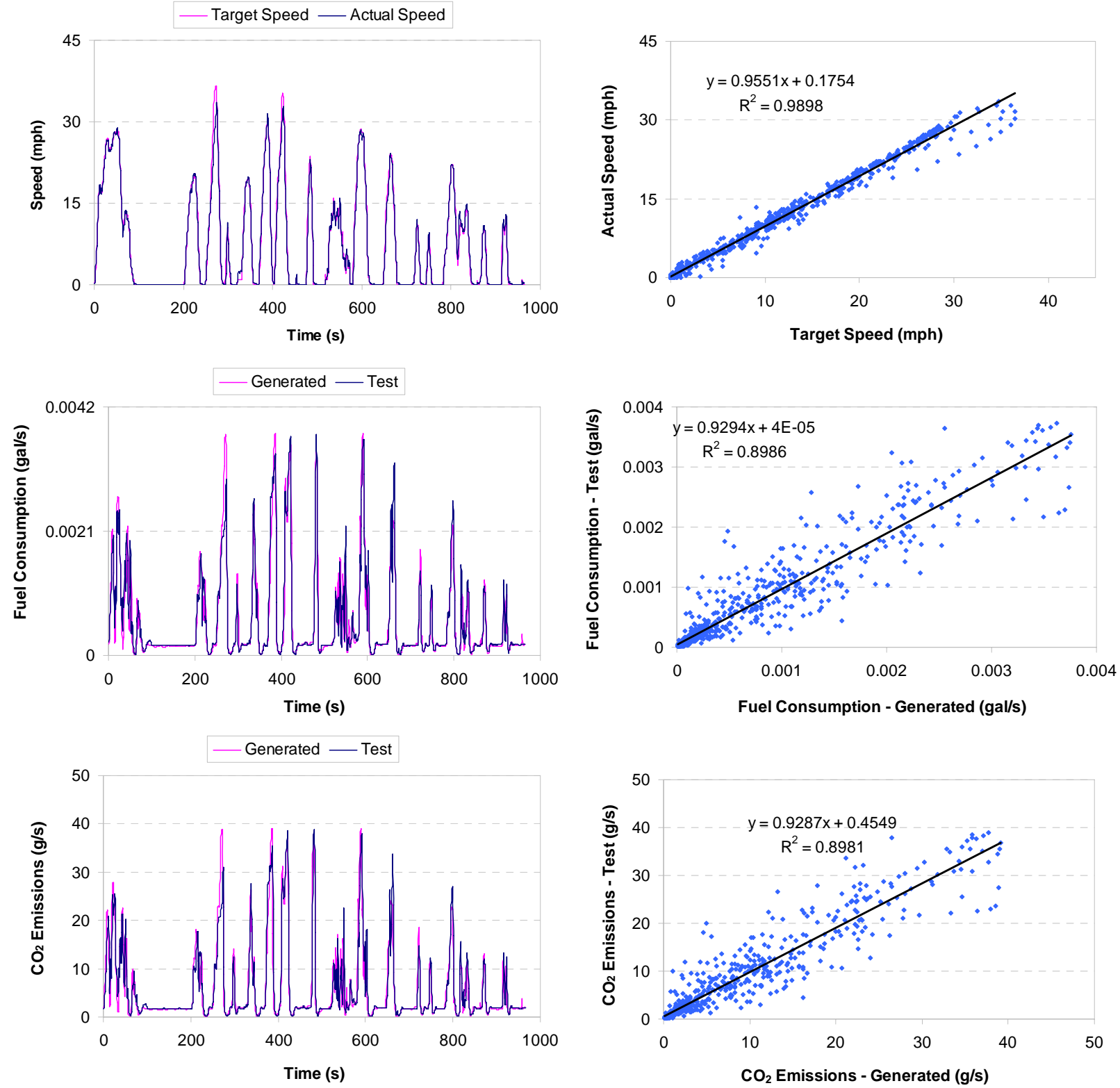

Figure B-7 Target and Actual Vehicle Speed, Fuel Consumption and $\mathrm{CO}_{2}$ Rates from Emissions Testing and Cycle Generation of the VC_28 from a MY 2008 Hybrid Bus 

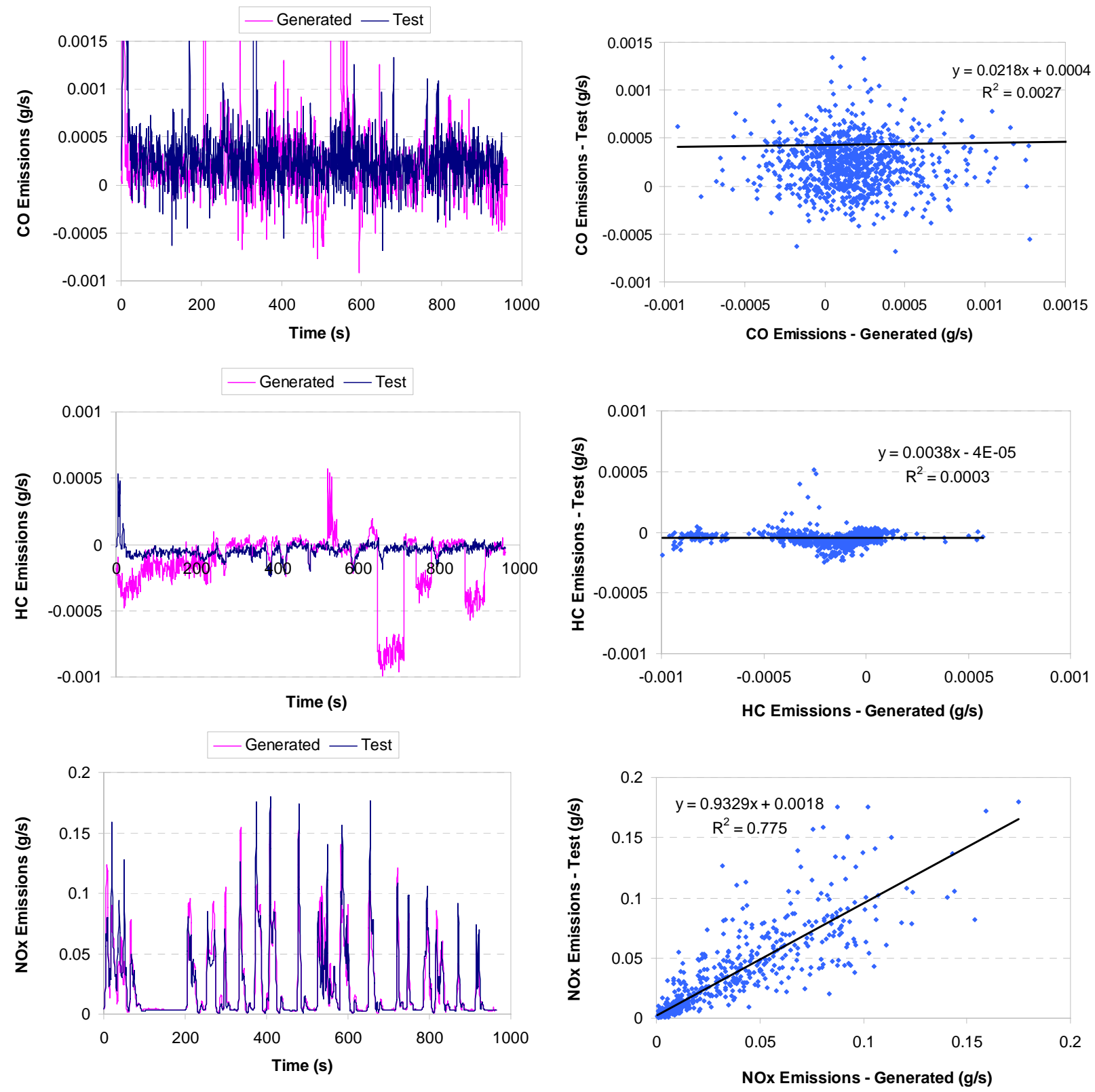

Figure B-8 CO, HC and NOx Rates from Emissions Testing and Cycle Generation of the VC_28 from a MY 2008 Hybrid Bus 